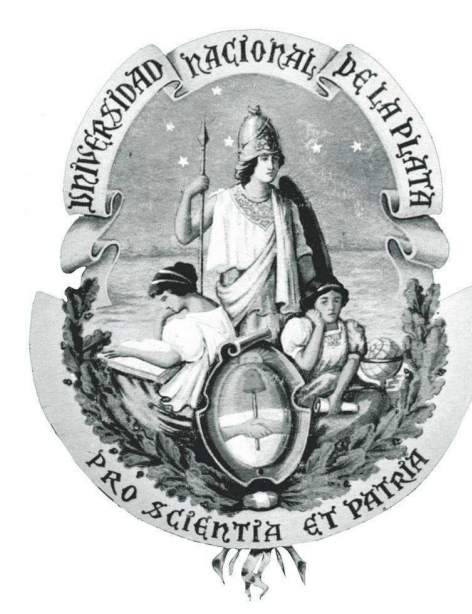

Universidad Nacional de La Plata

Facultad de Ciencias Exactas

Departamento de Física

Tesis presentada para optar al grado de

Doctor de la Facultad de Ciencias Exactas.

\title{
Modelos quirales para las interacciones fuertes: fenomenología de mesones y transiciones de fase en presencia de campos magnéticos externos
}

María Florencia Izzo Villafañe

Director: Dr. Daniel Gómez Dumm

Año 2017 



\section{Resumen}

La dinámica de quarks se encuentra descrita en el marco del Modelo Estándar por la Cromodinámica Cuántica (QCD), que formalmente es una teoría de campos de gauge no abeliana.

En procesos de altas energías la propiedad de libertad asintótica presente en QCD permite obtener predicciones a partir del lagrangiano fundamental de la teoría. Sin embargo, a bajas energías $(\lesssim 1 \mathrm{GeV})$ la constante de acoplamiento fuerte aumenta su valor de modo tal que las técnicas perturbativas ya no son aplicables. En este régimen los quarks se encuentran confinados en hadrones, y la simetría quiral se encuentra espontáneamente rota.

El empleo de teorías efectivas permite estudiar la fenomenología de las partículas hadrónicas y analizar el comportamiento de la materia fuertemente interactuante en sistemas con temperatura y densidad finitas. En esta Tesis se estudiaron modelos efectivos para la descripción de la interacción fuerte a bajas energías. En particular, se consideraron modelos de quarks relativistas del tipo Nambu-Jona-Lasinio con interacciones no locales para dos sabores de quarks de modo de estudiar la fenomenología de los mesones livianos. Se estudió también el acoplamiento con campos magnéticos externos uniformes, y el efecto de éstos sobre las propiedades de los mesones y las transiciones de fase que sufre la materia hadrónica a temperatura finita.

El presente trabajo está estructurado de la siguiente manera:

En el Capítulo 1 se presenta una breve introducción que describe el comportamiento a bajas energías de la materia fuertemente interactuante indicando los problemas que implica lidiar con QCD en la vecindad de las transiciones de fase. Se introducen dos de las principales alternativas para hacerlo: Lattice QCD (LQCD) y modelos efectivos, en particular, el propuesto por Nambu y Jona-Lasinio (NJL). También se discuten los resultados que motivan el estudio del efecto de campos magnéticos sobre la materia de quarks.

En el Capítulo 2 se discuten las características principales de la Cromodinámica Cuántica relevantes para la construcción de los modelos efectivos que serán utilizados, prestando especial atención a las simetrías de sabor y las simetrías de gauge.

En el Capítulo 3 se presenta el modelo de Nambu-Jona-Lasinio en su versión local de 
dos sabores, detallando los cálculos del condensado quark-antiquark y de las propiedades mesónicas del sector escalar y pseudoescalar mediante el formalismo de bosonización, para luego incorporar interacciones no locales y acoplamientos que den cuenta de la renormalización de la función de onda.

En el Capítulo 4 se extiende el modelo NJL no local incorporando acoplamientos entre corrientes vectoriales y axiales. En este marco se estudian las características del sector de mesones vectoriales livianos, con especial atención en el estudio de los mesones $\rho$ y $\pi$ en el vacío.

El Capítulo 5 se concentra en el procedimiento por el cual se incluye un acoplamiento con un campo magnético externo. Se incorpora dicho campo mediante la derivada covariante, indicando cómo debe realizarse el cálculo del determinante fermiónico correspondiente a la acción en la aproximación de campo medio. Luego, se desarrolla la acción efectiva a órdenes superiores en las fluctuaciones de los campos a fin de encontrar expresiones analíticas para las masas de los mesones $\pi$ y $\sigma$, así como el ancho de decaimiento débil del pion, en función de la magnitud del campo magnético.

En el Capítulo 6 se comienza explicando el formalismo de tiempo imaginario para incorporar temperatura finita, teniendo en cuenta a su vez el acoplamiento con el "Loop de Polyakov" (PL). En la segunda sección del capítulo se incorpora este formalismo al modelo descrito en el Cap. 5 para estudiar las transiciones de fase en función de la temperatura. En ambos casos se calculan los condensados quirales y la traza del loop de Polyakov en función de la magnitud del campo magnético y la temperatura, comparando nuestros resultados con los obtenidos en LQCD.

En el Capítulo 7, finalmente, se resume el trabajo realizado y se exponen las conclusiones. 


\section{Índice general}

Resumen III

$\begin{array}{ll}\text { 1. Introducción } & 1\end{array}$

2. Generalidades sobre simetrías y QCD 9

2.1. Simetrías . . . . . . . . . . . . . . . . . . . . 9

2.1.1. Simetrías exactas y aproximadas . . . . . . . . . . . 10

2.1.2. Simetrías de Gauge . . . . . . . . . . . . . . . . . . . . . . . . 11

2.1.3. Simetría quiral . . . . . . . . . . . . . . . . . 13

2.2. Ruptura espontánea de simetrías . . . . . . . . . . . . . . . . . . . 15

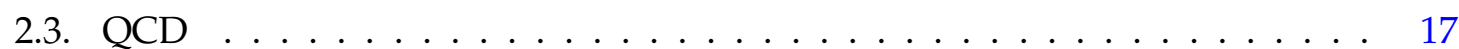

3. Modelo de Nambu-Jona-Lasinio 23

3.1. Introducción al modelo NJL . . . . . . . . . . . . . . . . . . . . 23

3.1.1. Generación dinámica de la masa fermiónica . . . . . . . . . . . . . . 24

3.1.2. Bosón de Goldstone pseudoescalar . . . . . . . . . . . . . . . . . 26

3.2. Extensiones no locales del modelo NJL . . . . . . . . . . . . . . . . . . . . . . 29

3.2.1. Interacciones no locales . . . . . . . . . . . . . . . . . . . . . . . . . 29

3.2.2. Modelo quiral no local de quarks con renormalización de la función de onda . . . . . . . . . . . . . . . . . . . . 31

3.2.3. Aproximación de campo medio . . . . . . . . . . . . . . . . . 33

3.2.4. Fluctuaciones cuadráticas . . . . . . . . . . . . . . . . . 35

3.2.5. Decaimiento débil del pion . . . . . . . . . . . . . . . . 37

4. Modelo NJL no local con acoplamientos vectoriales y vectoriales-axiales 39

4.1. Construcción del modelo . . . . . . . . . . . . . . . . . . . . . . . . . . . . . 39

4.1.1. Aproximación de campo medio . . . . . . . . . . . . . . . . . . . . . 39

4.1.2. Fluctuaciones cuadráticas . . . . . . . . . . . . . . . . . . . 42

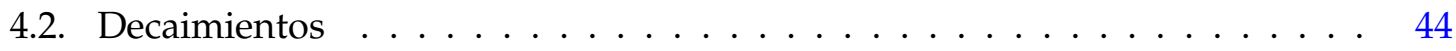


4.2.1. Decaimiento débil del pion . . . . . . . . . . . . . . . 44

4.2.2. Vértice $\rho^{0}$-fotón y constante de decaimiento electromagnética del $\rho .45$

4.2.3. Decaimiento $\pi^{0} \rightarrow \gamma \gamma \ldots \ldots \ldots \ldots$. . . . . . . . 48

4.2.4. Decaimiento $\rho \rightarrow \pi \pi \ldots \ldots \ldots \ldots \ldots$. . . . . . . . . . . . . . . . 50

4.3. Factores de forma y parámetros del modelo . . . . . . . . . . . . . . 52

4.4. Resultados numéricos . . . . . . . . . . . . . . . . 56

5. Modelo NJL no local en presencia de campo magnético 59

5.1. Interacciones magnéticas en el modelo NJL no local . . . . . . . . . . . 59

5.1.1. Formalismo en la aproximación de campo medio . . . . . . . . . . 60

5.1.2. Constantes fenomenológicas para los mesones $\sigma \mathrm{y} \pi^{0} \ldots \ldots 6$

5.2. Parametrizaciones . . . . . . . . . . . . . . . . . . 73

5.3. Resultados numéricos . . . . . . . . . . . . . . . . . . . 74

6. Modelo NJL no local a temperatura finita en presencia de campo magnético 81

6.1. Modelo NJL local a temperatura finita . . . . . . . . . . . . . . . 81

6.1.1. Formalismo de Matsubara o de tiempo imaginario . . . . . . . . . . . 81

6.1.2. Loop de Polyakov . . . . . . . . . . . . . . . . . . . 83

6.1.3. Modelo Polyakov-Nambu-Jona Lasinio (PNJL) . . . . . . . . . . . . . 86

6.2. Modelo PNJL no local con campo magnético a temperatura finita . . . . . 88

6.2.1. Resultados numéricos para temperatura finita . . . . . . . . . . . 90

$\begin{array}{ll}\text { 7. Resumen y conclusiones } & 97\end{array}$

$\begin{array}{ll}\text { A. Bosonización } & 103\end{array}$

B. Integrales a un loop y factores de forma en el plano complejo 105

$\begin{array}{ll}\text { C. Autofunciones y transformaciones de Ritus } & 109\end{array}$

$\begin{array}{ll}\text { D. Cálculo de } G_{\bar{p}, \bar{p}^{\prime}}^{\lambda, f} & 113\end{array}$

$\begin{array}{ll}\text { E. Cálculo del propagador de quark a campo medio } & 117\end{array}$

F. Cálculo de la masa del pion en presencia de campo magnético 121

G. Cálculo de la constante de decaimiento del pion en presencia de campo magnético 


\section{Capítulo 1}

\section{Introducción}

De acuerdo con el Modelo Estándar de las partículas elementales [1] los hadrones son estados ligados de fermiones llamados quarks, que interactúan entre sí a través de bosones mediadores llamados gluones. La teoría que describe dichas interacciones es una teoría de gauge no abeliana llamada Cromodinámica Cuántica (o QCD, por Quantum Chromodynamics) [2, 3]. En esta teoría, tanto los quarks como los gluones poseen una propiedad conocida como "carga de color", análoga a la carga eléctrica de la electrodinámica, que es la responsable de las interacciones fuertes. Uno de los objetivos de QCD es describir adecuadamente cómo los quarks y los gluones interactúan para formar los estados ligados que conforman la materia hadrónica, los cuales se clasifican en bariones y mesones.

El hecho de que el grupo de simetría interna SU(3) de color sea no abeliano hace que QCD tenga características muy diferentes a las de la Electrodinámica Cuántica. En particular, QCD tiene dos propiedades sumamente importantes: libertad asintótica y confinamiento. La libertad asintótica implica que en procesos en los cuales el momento transferido es muy grande, tales como dispersiones profundamente inelásticas, los quarks se comportan como casi libres, es decir, la interacción entre dos quarks debida al intercambio de gluones resulta ser muy pequeña. Esto permite obtener predicciones a partir del lagrangiano fundamental de la teoría utilizando teoría de perturbaciones, esto es, a través de un desarrollo en serie de potencias de la constante de acoplamiento quark-gluon, partiendo de un sistema de quarks no interactuantes. Por el contrario, para bajos momentos transferidos $(\lesssim 1 \mathrm{GeV})$ QCD es altamente no lineal y da lugar al confinamiento, lo cual significa que los quarks no existen como partículas libres sino que forman estados ligados debido a que la intensidad de interacción entre ellos aumenta con la distancia. Este fenómeno se refleja en que las variables de color se acomodan necesariamente en estados conocidos como "singuletes", cuya carga neta de color es nula y que requieren de más de un quark o antiquark para formarse. 
Por otro lado, también son de importancia las propiedades de simetría ante transformaciones globales en el espacio de sabor. El lagrangiano de QCD está construido de manera de ser aproximadamente simétrico ante los grupos $\mathrm{SU}(2)$ de isospín y $\mathrm{SU}(2)$ axial en el sector de quarks livianos. Sin embargo, en el estado de vacío de QCD la simetría axial se rompe espontáneamente debido a las interacciones, pudiendo caracterizarse la fase correspondiente mediante parámetros de orden como el condensado quiral, el cual está a su vez relacionado con la generación dinámica de masa. De hecho, la ruptura espontánea de la simetría axial de QCD es la responsable de la mayor parte de la masa de los nucleones.

Debido a estas características, poco después del advenimiento de QCD se conjeturó que a temperaturas y/o densidades suficientemente elevadas podrían existir nuevos estados de materia, conocidos con el nombre genérico de plasma de quarks y gluones (QGP) [4, 5], que se encuentran caracterizados por el deconfinamiento de color. Esto motivó el estudio teórico de las fases posibles de QCD en tales condiciones extremas y reveló una estructura de fases potencialmente compleja, cuya descripción continúa resultando hasta el día de hoy un desafío tanto teórico como experimental [6,7, 8].

Los efectos dinámicos de las rupturas de simetrías son de gran importancia en el estudio del diagrama de fases de QCD ya que existen indicios de que la simetría axial se encuentra restaurada en la fase QGP. Además, las evidencias experimentales y teóricas conducen a suponer que las transiciones de fase de deconfinamiento y de restauración de simetría quiral ocurren en forma prácticamente simultánea a densidades bajas, y que el pasaje de una fase a otra tiene lugar a través de una transición suave del tipo crossover $[9,10]$. Sin embargo, el mecanismo preciso que da lugar a esta transición simultánea aún no ha sido comprendido en su totalidad.

Se cree que en la Naturaleza el plasma de quarks y gluones existió en el universo temprano, en los primeros instantes posteriores al Big Bang, cuando la temperatura era muy elevada, y que luego, a medida que el Universo se fue enfriando, tuvo lugar el proceso de hadronización. Hoy en día se considera que es posible que aún exista alguna forma de materia de quarks deconfinados en el interior de objetos compactos [11] como las estrellas de neutrones, cuyo interior profundo correspondería a la región de altas densidades y bajas temperaturas en el diagrama de fases. Las estrellas compactas [12] son objetos extremadamente estables y densos, y constituyen uno de los posibles escenarios finales en el ciclo de vida de una estrella. Al producirse una supernova se eyecta al espacio gran parte de la materia que constituye la estrella y su núcleo se comprime fuertemente. El remanente de dicha explosión puede convertirse en una estrella compacta cuando la masa del objeto se encuentra por debajo del límite de Chandrasekhar. En esta etapa de su ciclo de vida ya no se producen reacciones de fusión, y la fuerza gravitatoria es equilibrada por una presión de origen cuántico debida al principio de exclusión de Pauli. 
El fuerte interés por estudiar las propiedades de la materia de quarks (o el QGP) y las condiciones bajo las cuales es posible producirla ha motivado en los últimos años la construcción de grandes instalaciones experimentales que permitan obtenerla en el laboratorio. Una de ellas es el Relativistic Heavy Ion Collider (RHIC) en Brookhaven, USA [13], donde se llevan a cabo estudios relacionados con el plasma de quark y gluones a través de colisiones entre iones pesados a energías relativistas. También en el Large Hadron Collider ubicado en el CERN en la frontera franco-suiza cerca de Ginebra se realizan experimentos de este tipo, estando el detector Alice especialmente dedicado a este fin [14]. Además de éstas, existen otras instalaciones en construcción, el Facility for Antiproton and Ion Research (FAIR) [15] en Darmstadt, Alemania, y el NICA [16] en Dubna, Rusia.

En este tipo de aceleradores el objetivo es colisionar iones pesados como plomo, plata u oro a energías de centro de masa del orden de 100 - 200 GeV o más. Durante el proceso de colisión se alcanzan temperaturas extremadamente altas, por encima de la temperatura crítica esperada de deconfinamiento, $T_{\mathcal{c}} \simeq 170 \mathrm{MeV}$ [17]. Los resultados actuales sugieren que el QGP ha logrado formarse [18, 19] durante períodos de tiempo del orden de $10 \mathrm{fm} / \mathrm{c}$, incluso exhibiendo propiedades de equilibrio termodinámico a pesar de su corta duración, y además dando evidencias que confirman la hipótesis de que la transición entre las fases hadrónica y QGP es de tipo crossover.

Es importante destacar que las condiciones producidas en aceleradores de iones pesados corresponden esencialmente a la zona de baja densidad del diagrama de fases. $\mathrm{Si}$ bien en el FAIR se espera que se puedan alcanzar densidades algo mayores, los efectos de densidad finita más relevantes están aún lejos de observarse en este tipo de experimentos, relegando la posibilidad de observar fases densas al estudio de estrellas compactas.

Para poder describir la materia hadrónica y de quarks, así como las transiciones de fase, es esencial disponer de métodos que permitan analizar la dinámica asociada a QCD. Si bien se conoce la teoría fundamental que describe toda la dinámica del sistema, para procesos físicos de bajas energías resulta muy difícil obtener información relevante partiendo en forma directa de ella. Para describir las propiedades estáticas de los hadrones, tales como sus masas, constantes de decaimiento, etc., así como para estudiar la transición entre la fase hadrónica y la de materia de quarks, es necesario considerar el rango de momentos transferidos pequeños, y resulta fundamental desarrollar formalismos que permitan estudiar la teoría en esta región mediante técnicas no perturbativas. Un método posible consiste en resolver numéricamente las ecuaciones de movimiento en un espacio-tiempo discretizado, lo que se denomina "QCD en la red" o Lattice QCD (LQCD) [20]. Disponiendo de suficiente poder de cómputo es posible disminuir el espaciamiento de la red e incrementar el tamaño del sistema de manera de acercarse tanto al límite del continuo como al termodinámico. Al mismo tiempo es posible muestrear un número suficientemente grande de configuraciones 
de manera de disminuir el error estadístico. Sin embargo, los métodos tipo Monte Carlo comúnmente utilizados para evaluar la función de partición son confiables solamente en el caso en el que el potencial químico es cero [21]. La extensión de los cálculos a potencial químico finito presenta grandes dificultades, y si bien existen algunos resultados, éstos se encuentran aún en discusión.

Otra de las maneras posibles de encarar el estudio de QCD a bajas energías consiste en desarrollar modelos efectivos. Un modelo efectivo constituye una simplificación de la teoría completa de modo de facilitar su análisis, y a la vez contiene en su formulación ciertos ingredientes básicos de la teoría original que permiten reproducir aspectos específicos y realizar predicciones cuantitativas de interés. Una ventaja de los modelos efectivos frente a LQCD es que pueden ser extendidos a potencial químico finito sin mayor dificultad, y de este modo permiten estudiar en forma completa el diagrama de fases. Entre los varios modelos posibles se encuentran el MIT Bag model [22], el modelo sigma lineal [23] y el que se usará como punto de partida en esta Tesis, el modelo de Nambu-Jona-Lasinio (NJL).

El modelo NJL fue desarrollado en el año 1961, en el que Y. Nambu y G. Jona-Lasinio publicaron dos trabajos [24, 25] cuyo objetivo era estudiar las interacciones entre nucleones, con el propósito de explicar en forma unificada las grandes masas de los bariones y las masas intermedias o pequeñas de los mesones (en particular, la masa pequeña que distingue al pion de los demás hadrones) a partir de las propiedades de simetría de isospín que exhiben las interacciones nucleares. En sus trabajos Nambu y Jona-Lasinio partieron de un lagrangiano con una interacción de dos cuerpos entre nucleones (los bloques básicos de materia conocidos hasta ese momento) no masivos que respeta la simetría quiral. Dicha invarianza se rompe debido a los efectos de las interacciones. Este mecanismo produce que los campos fundamentales que representan a los nucleones adquieran masas dinámicas, dando lugar también a bosones de Goldstone, los cuales son identificados con los piones.

Por esa época QCD aún no había sido formulada. Hacia mediados de los años 70, cuando los quarks fueron reconocidos como los componentes elementales de la materia hadrónica en lugar de los protones y neutrones, el modelo NJL empezó a ser abandonado, debido a su naturaleza no fundamental y a su no renormalizabilidad. Sin embargo, como consecuencia de las dificultades antes mencionadas para tratar QCD a bajas energías, en la segunda parte de la década del 80 surgió la idea de reinterpretar el modelo NJL como un modelo para un sistema de quarks interactuantes. Se supone así que los grados de libertad de los gluones se pueden "congelar" dando lugar a interacciones efectivas entre los quarks.

En su versión más sencilla, el lagrangiano del modelo NJL contiene términos locales de interacción del tipo escalar-isoescalar y pseudoescalar-isovectorial entre fermiones, que 
son los que reproducen la dinámica de ruptura de simetría quiral de QCD. Este modelo, así como sus extensiones que incluyen interacciones locales en otros canales, ha sido utilizado en numerosos estudios de las propiedades hadrónicas, tanto considerando simetrías $\mathrm{SU}(2)$ como SU(3) de sabor (incluyendo los quarks up, down y strange), y a su vez ha permitido analizar la respuesta de la materia de quarks ante efectos de temperatura, potencial químico [26, 27, 28] y campo magnético [29]. También ha sido uno de los modelos más usados para investigar la existencia de fases superconductoras de color [30]. Sin embargo, para dar cuenta del mecanismo de deconfinamiento no basta con un modelo puramente fermiónico sino que es necesario incluir en forma explícita la dinámica de los grados de libertad gluónicos. Recientemente ha sido propuesto que éstos sean incorporados mediante el acoplamiento de los quarks a un campo de fondo de color asociado al parámetro de orden usualmente utilizado para describir el deconfinamiento, esto es, el llamado loop de Polyakov [31, 32]. El modelo así obtenido se conoce bajo el nombre de modelo de PolyakovNambu-Jona-Lasinio (PNJL) [33, 34, 35].

Un problema del modelo NJL, relacionado con el uso de interacciones locales, es que se debe introducir algún tipo de regularización para evitar que los diagramas involucrados en la determinación de la autoenergía de los quarks, las masas de los mesones, las constantes de acoplamiento, etc., resulten divergentes. Esto introduce ciertas ambigüedades en el cálculo de dichas cantidades, así como de las correcciones debidas a los loops de mesones. A partir de los 90's han aparecido trabajos que proponen el uso de interacciones no locales para solucionar este tipo de inconvenientes, a cambio de algunas complicaciones en el cálculo. En verdad, el carácter no local de las interacciones surge naturalmente en el contexto de diversos métodos bien establecidos para describir la dinámica a bajas energías de los quarks, como por ejemplo el modelo de líquido de instantones [36] o las técnicas de resumación de Schwinger-Dyson [37]. Una de las propuestas de mayor interés surge de la relación entre el modelo NJL y el modelo de intercambio de un gluon, según el cual se utiliza el propagador de un gluon modificado fenomenológicamente para generar las interacciones efectivas entre quarks. El propagador gluónico efectivo provee una forma natural de introducir una no localidad dentro de la interacción quark-quark [38], y dicha no localidad se puede caracterizar mediante un factor de forma $g(p)$, que depende del modelo. El uso de interacciones no locales tiene diversas ventajas ya que, por ejemplo, las anomalías son automáticamente preservadas [39], y la acción efectiva resulta finita a todo orden en el desarrollo en loops [40], sin necesidad de introducir parámetros adicionales. Por otro lado, mediante una adecuada elección del factor de forma es posible lograr que el propagador fermiónico no tenga polos reales y que, por lo tanto, los quarks no puedan aparecer como estados asintóticos. Esto ha sido propuesto [41] como una forma de implementar la propiedad de confinamiento que posee QCD, lo que en el modelo NJL local no 
es posible hacer. Más aún, utilizando este tipo de interacciones es posible obtener dependencias en el momento del propagador efectivo de quarks que son consistentes con las obtenidas mediante técnicas de LQCD.

En este marco, uno de los objetivos de esta Tesis es investigar el comportamiento de la materia hadrónica proponiendo una extensión del modelo NJL que incluya acoplamientos no locales entre corrientes vectoriales y axiales que satisfacen las simetrías de QCD. Asimismo, consideraremos una interacción no local que conduce a la renormalización de función de onda (WFR, Wave Function Renormalization) de los quarks. Ésta permitirá obtener una descripción del propagador de quarks similar a la que resulta de los cálculos de LQCD, y realizar comparaciones cuantitativas con los resultados obtenidos en ese formalismo.

Otro objetivo es estudiar, mediante los modelos no locales, el efecto de campos magnéticos intensos sobre el comportamiento de materia fuertemente interactuante. Estudios de este tipo se encuentran motivados por el hecho de que existen indicios claros de que tanto en las colisiones de iones pesados como en los núcleos de estrellas compactas se producen campos magnéticos extremadamente elevados. Por ejemplo, en el RHIC, en una colisión típica, los campos magnéticos pueden alcanzar los $10^{19} \mathrm{G}\left(\simeq 0,06 \mathrm{GeV}^{2}\right.$ en unidades naturales) $[42,43]$ en la región central. En estrellas compactas, por otra parte, los campos magnéticos pueden ser tan grandes como $10^{15} \mathrm{G}$ [44] en su superficie, y alcanzar valores en el rango de $10^{18}-10^{20} \mathrm{G}$ en el núcleo $[45,46,47]$, aunque las estimaciones para estos últimos valores son dependientes del modelo utilizado. Por ello, es interesante estudiar cuáles podrían ser los efectos de campos magnéticos intensos sobre la materia de quarks, $y$, en particular, determinar si el diagrama de fases sufre modificaciones considerables. Uno de los efectos más discutidos es el de "Catálisis Magnética" [48], que se refiere a la estabilización del condensado quiral en la fase hadrónica al aplicar un campo magnético externo. La interpretación básica del fenómeno es que dicho campo favorece el antialineamiento de los espines del quark y el antiquark que están correlacionados en un condensado. Si bien la realización de este fenómeno en la fase de vacío se encuentra relativamente comprendida, a temperatura finita y densidades bajas existen comportamientos más difíciles de explicar en forma cualitativa. De hecho, existen cálculos realizados mediante LQCD donde se observa que a temperaturas intermedias (del orden de $150 \mathrm{MeV}$ ) el condensado quiral disminuye con el campo, contrariamente a lo esperado. Esto a su vez trae aparejada una disminución en dicha región de la temperatura crítica de transición de fase quiral. Este comportamiento, conocido como "Catálisis Magnética Inversa" [49], es difícil de reproducir mediante modelos efectivos y, en particular, modelos como el NJL local predicen la tendencia exactamente contraria, es decir, un aumento de la temperatura crítica con el campo magnético $[48,50,51]$. A densidad finita y temperaturas bajas, por otro lado, existe 
otra manifestación del fenómeno de catálisis magnética inversa, el cual está asociado a la disminución del potencial químico crítico como función del campo magnético. 



\section{Capítulo 2}

\section{Generalidades sobre simetrías y QCD}

En este capítulo detallaremos algunos de los aspectos básicos del Modelo Estándar (ME) que resultan relevantes para el desarrollo de este trabajo. En principio nos enfocaremos en la noción de simetría dentro de la teoría cuántica de campos, y las simetrías internas en la física de partículas elementales que consideramos de mayor importancia en el rango de energía en el cual desarrollaremos el modelo NJL.

Asimismo, haremos un repaso breve de la Cromodinámica Cuántica: su formulación teórica, sus propiedades más características y las predicciones exitosas de la teoría, y concluiremos con sus limitaciones y los métodos utilizados hoy en día para sortearlas.

\subsection{Simetrías}

Actualmente el ME representa el marco teórico fundamental para describir los fenómenos dentro de la física de partículas. Este modelo es una teoría relativista de campos cuánticos, que describe la estructura fundamental de la materia y el vacío considerando las partículas elementales como entes irreducibles cuya cinemática está regida por las interacciones entre ellas. Dentro del ME se describen exitosamente tres de las cuatro fuerzas fundamentales: la fuerza electromagnética, la fuerza débil y la fuerza fuerte. A su vez, el modelo permite clasificar todas las partículas elementales conocidas hasta el momento.

Los fermiones elementales pueden dividirse en dos grandes categorías de acuerdo a cómo interaccionan entre sí: leptones y quarks. Los quarks no se observan en forma aislada sino que interactúan fuertemente quedando confinados en hadrones: mesones y bariones, más los hipotéticos tetraquarks, pentaquarks y moléculas hadrónicas. A los seis leptones y los seis sabores de quarks conocidos hasta el momento se los puede agrupar en tres generaciones o familias de dos partículas cada una.

Por otro lado, el ME explica las fuerzas que se observan a nivel macroscópico como el resultado de un intercambio de partículas conocidas como las mediadoras de la fuerza. Las 
partículas mediadoras son de naturaleza bosónica (los fotones, los bosones $W^{ \pm}$y $Z^{0}$, y los gluones), y por lo tanto no siguen el principio de exclusión de Pauli.

La teoría cuántica de campos (QFT por Quantum Field Theory) provee el contexto matemático para el desarrollo del ME, a partir de proponer un lagrangiano que determina la dinámica de la teoría, y donde cada tipo de partícula es descrita en términos de un campo dinámico definido sobre el espacio-tiempo. La construcción del ME se realiza del modo en que lo hacen las mayoría de las teorías de campos, postulando primero un número de simetrías del sistema y luego escribiendo el lagrangiano renormalizable más general para los campos que satisfagan dichas simetrías.

Es por ello que resulta clave para comprender las interacciones fundamentales estudiar y conocer a fondo las simetrías que éstas presentan.

\subsubsection{Simetrías exactas y aproximadas}

Se dice que existe una simetría en la naturaleza cuando algún cambio en las variables de un sistema deja la física fundamental intacta. Las simetrías describen invarianzas ante transformaciones de los campos cuánticos. Por ejemplo, una traslación en el espacio del tipo $x_{\mu} \rightarrow x_{\mu}+a_{\mu}$ implica una transformación en un campo $\phi(x)$ escalar de modo que $\phi(x) \rightarrow \phi(x+a)$.

El análisis de simetrías en la teoría de campos se enfoca en el lagrangiano, que es una función escalar dependiente de varios campos $\varphi_{i}$ y sus primeras derivadas $\partial_{\mu} \varphi_{i}$. A partir del lagrangiano, o de su integral temporal denominada acción $S$, se puede describir la dinámica de las partículas y las ecuaciones de movimiento del sistema en que se esté trabajando. Asimismo, el Teorema de Noether afirma que para cualquier invarianza de la acción ante una transformación continua de los campos, existe una carga $Q$ que es independiente del tiempo $(\dot{Q}=0)$ y está asociada con una corriente conservada $\left(\partial_{\mu} J^{\mu}=0\right)$. Este teorema cubre tanto las simetrías internas como las espacio-temporales.

Las consideraciones sobre las simetrías son también útiles en situaciones donde hay una simetría aproximada. Puede suceder que un lagrangiano presente una simetría en el límite en que ciertos parámetros en él son iguales a cero o iguales entre sí. En ese límite la invarianza tendría una serie de consecuencias físicas que no se podrían obtener si esos mismos parámetros tuvieran valores no nulos, o si la diferencia entre ellos fuera distinta de cero. Sin embargo, si éstos son en algún sentido "pequeños" los términos no invariantes pueden ser tratados como una perturbación y las consecuencias predichas son todavía aproximadamente válidas. De hecho, cuando la interacción que rompe la invarianza tiene un comportamiento bien definido bajo la transformación de simetría correspondiente, su efecto puede ser generalmente analizado en términos de la base de estados de partículas 
no perturbadas utilizando el teorema de Wigner-Eckart. El sentido preciso en el cual los términos de ruptura de simetría pueden ser considerados pequeños depende del problema en cuestión. En la práctica, la utilidad de una simetría aproximada raramente se conoce $a$ priori, pero es evidente luego de que sus predicciones son corroboradas experimentalmente. Si la simetría no es exacta entonces, en principio, las corrientes asociadas y las cargas no se conservarán.

También es posible encontrar las llamadas anomalías cuánticas, las cuales hacen referencia a simetrías de langragianos clásicos para las cuales la corriente asociada no resulta conservada a nivel cuántico.

\subsubsection{Simetrías de Gauge}

El lagrangiano de Dirac, en ausencia de más términos, describe la propagación de quarks libres. Éste viene dado por

$$
\mathcal{L}=i \bar{\psi} \gamma^{\mu} \partial_{\mu} \psi-m \bar{\psi} \psi
$$

y es invariante ante transformaciones del tipo $\psi \rightarrow e^{-i \theta} \psi$, ya que $\bar{\psi} \rightarrow e^{i \theta} \bar{\psi}$, y en la combinación $\bar{\psi} \psi$ los factores exponenciales se cancelan. Por razones históricas estas transformaciones son llamadas transformaciones de gauge globales, o transformaciones de fase global.

Sin embargo, también pueden construirse transformaciones de fase o de gauge locales, para las cuales $\theta$ es un parámetro con dependencia en las coordenadas espacio-temporales, y por lo tanto el campo transforma como $\psi(x) \rightarrow \exp [-i \theta(x)] \psi(x)$. A la hora de aplicar la transformación local al lagrangiano de Dirac, éste ya no mantiene la invarianza de gauge. En particular, términos cinéticos como $i \bar{\psi} \gamma^{\mu} \partial_{\mu} \psi$, generan la aparición de un término adicional proporcional a $\partial_{\mu} \theta(x)$

$$
i \bar{\psi}(x) \gamma^{\mu} \partial_{\mu} \psi(x) \rightarrow i \bar{\psi} \gamma^{\mu} \partial_{\mu} \psi(x)+\bar{\psi}(x) \gamma^{\mu} \psi(x) \partial_{\mu} \theta(x) .
$$

Para lograr que el lagrangiano sea un invariante de la transformación local se necesita reemplazar la derivada parcial por una derivada $D_{\mu}$ extendida que transforme de tal modo que absorba el nuevo término derivativo y cumpla que

$$
D_{\mu} \psi(x) \rightarrow \exp [-i \theta(x)] D_{\mu} \psi(x) .
$$

A este nuevo operador $D_{\mu}$ se lo denomina derivada covariante, y para su construcción se necesitan nuevos campos $A_{\mu}$, llamados campos de gauge, que frente a las transformaciones locales mantengan la invarianza del lagrangiano cancelando los términos proporcionales a $\partial_{\mu} \theta(x)$. Para ello se define la transformación de gauge local de los campos $A_{\mu}$, como

$$
A_{\mu} \rightarrow A_{\mu}+\partial_{\mu} \theta(x)
$$


y la derivada covariante vendrá dada por

$$
D_{\mu} \equiv \partial_{\mu}-i A_{\mu}(x)
$$

De este modo estamos agregando un nuevo campo en la dinámica de nuestro sistema. Para que el campo $A_{\mu}$ sea dinámico y podamos ahora también calcular sus correspondientes ecuaciones de movimiento debemos incluir un término cinético, que sea invariante de gauge y de Lorentz. Esto se logra definiendo $F_{\mu v}=\partial_{\mu} A_{\nu}-\partial_{\nu} A_{\mu}$ y agregando al lagrangiano un término proporcional a $F_{\mu \nu} F^{\mu \nu}$,

$$
\mathcal{L}=i \bar{\psi} \gamma^{\mu} D_{\mu} \psi-m \bar{\psi} \psi-\frac{1}{4} F_{\mu v} F^{\mu \nu} .
$$

Observando el nuevo lagrangiano se descubre que si $A_{\mu}$ es el potencial electromagnético, los nuevos términos que se agregaron reproducen el lagrangiano de Maxwell y las interacciones electromagnéticas. Se podría decir entonces que la interacción es una manera en que la naturaleza impone la invarianza, y que las propiedades de la interacción electromagnética pueden obtenerse simplemente de exigir que se cumpla la simetría de gauge.

La transformación que hemos descrito corresponde al grupo U(1), es decir que es una transformación unitaria proporcional a la identidad. Yang y Mills implementaron la invarianza local para transformaciones pertenecientes a los grupos de simetría $\mathrm{SU}(\mathrm{N})$. En este caso, tenemos transformaciones del tipo

$$
U=\exp \left[-i \lambda_{a} \theta^{a}(x)\right]
$$

donde las matrices $\lambda_{a}$ con $a=1, \cdots, N^{2}-1$ pertenecen al conjunto de matrices unitarias generadoras del álgebra del grupo $\mathrm{SU}(\mathrm{N})$, y los $\theta^{a}(x)$ son los correspondientes parámetros dependientes de las coordenadas espacio-temporales asociados a los generadores. $\mathrm{Al}$ construir la derivada covariante para este caso se incorporan tantos campos de gauge como generadores tenga el correspondiente grupo de simetría (por ejemplo, tres en el caso de $\mathrm{SU}(2)$, ocho para SU(3), etc). La transformación de estos campos no es trivial. Las diferencias con el caso U(1) surgen de que en estos casos los grupos de simetría son no abelianos, y por lo tanto los generadores no conmutan. Entonces, se pueden definir dos tipo de teorías de gauge: las abelianas o de Weyl, y las no abelianas o de Yang-Mills.

En el contexto de la simetría SU(3) de color de las interacciones fuertes y la simetría $\mathrm{SU}(2) \otimes \mathrm{U}(1)$ de las interacciones electrodébiles la teoría de Yang-Mills toma un rol preponderante en la física de altas energías. Explicaremos más en profundidad este tipo de teorías en las secciones posteriores aplicado directamente al estudio de la simetría $\mathrm{SU}(3)_{c}$ de la cromodinámica cuántica. 


\subsubsection{Simetría quiral}

Se dice que los fermiones tienen quiralidad bien definida cuando sus espines están alineados (dextrógiros o "right") o anti-alineados (levógiros o "left") con sus impulsos. Si introducimos proyectores para ambas quiralidades, dados por

$$
P_{R}=\frac{1+\gamma_{5}}{2} \quad \text { y } \quad P_{L}=\frac{1-\gamma_{5}}{2},
$$

donde $\gamma_{5}$ es el operador de quiralidad, los campos dextrógiro, $\psi_{R}$, y levógiro, $\psi_{L}$, pueden construirse según

$$
\psi_{R}=P_{R} \psi, \quad \psi_{L}=P_{L} \psi, \quad \bar{\psi}_{R}=\psi_{R}^{\dagger} \gamma_{0}=\bar{\psi} P_{L}, \quad \bar{\psi}_{L}=\psi_{L}^{\dagger} \gamma_{0}=\bar{\psi} P_{R} .
$$

En esta base el lagrangiano libre de Dirac $\mathcal{L}_{0}$ correspondiente a fermiones sin masa toma la forma

$$
\mathcal{L}_{0}=i \bar{\psi}_{L} \gamma^{\mu} \partial_{\mu} \psi_{L}+i \bar{\psi}_{R} \gamma^{\mu} \partial_{\mu} \psi_{R}
$$

Como vemos, $\mathcal{L}_{0}$ se descompone en dos subespacios ortogonales left y right, que además se encuentran desacoplados entre sí. Este lagrangiano resulta invariante ante el grupo producto de transformaciones globales $\mathrm{U}(1)_{R} \otimes \mathrm{U}(1)_{L}$ dadas por

$$
U(1)_{L}: \psi_{L} \rightarrow \exp \left(-i \theta_{L}\right) \psi_{L}, \quad U(1)_{R}: \psi_{R} \rightarrow \exp \left(-i \theta_{R}\right) \psi_{R},
$$

con fases constantes arbitrarias $\theta_{L, R}$. Esta simetría es la llamada simetría quiral $U(1)_{L} \otimes U(1)_{R}$ cuyas transformaciones son proporcionales a la identidad.

Nos interesa analizar las propiedades del lagrangiano ante transformaciones en el espacio de sabor de los quarks. En el "límite quiral", donde $\hat{m}=0$, obtenemos el caso más simétrico posible. En general el término de masa del lagrangiano de Dirac no es invariante ante transformaciones unitarias arbitrarias en el espacio completo de sabor, en virtud de las grandes diferencias entre las masas de las distintas especies de quarks. Sin embargo, si nos restringimos al subespacio de sabores livianos, existen ciertas simetrías que se satisfacen en forma aproximada.

En este trabajo nos concentraremos únicamente en el subespacio de sabores up y down, cuyas masas se encuentran en el rango 4-10 MeV. Por lo tanto, estudiaremos la invarianza de $\mathcal{L}_{0}$ ante el grupo de transformaciones quirales de sabor $\mathrm{U}(2)_{L} \otimes \mathrm{U}(2)_{R}$ que viene dado por

$$
U(2)_{L}: \psi_{R} \rightarrow \exp \left(i \theta_{a}^{R} \tau_{a}\right) \psi_{R}, \quad U(2)_{R}: \psi_{L} \rightarrow \exp \left(i \theta_{a}^{L} \tau_{a}\right) \psi_{L},
$$

con $a=0,1,2,3$, donde cada una de éstas actúa independientemente sobre el subespacio correspondiente, rotando los estados en el espacio de sabor. Aquí, $\tau_{0}$ es la matriz identidad en espacio de sabor, mientras que $\tau_{k}$, con $k=1,2,3$, es el conjunto de matrices de 
Pauli y $\theta_{a}^{L, R}$ son los ángulos que parametrizan las transformaciones. Aplicando el teorema de Noether, la invarianza del lagrangiano ante estas transformaciones conduce a las siguientes corrientes conservadas

$$
\begin{aligned}
& \partial_{\mu} j_{R, a}^{\mu}=0 \\
& \partial_{\mu} j_{L, a}^{\mu}=0
\end{aligned} \quad \rightarrow \quad \begin{aligned}
& j_{R, a}^{\mu}=\bar{\psi}_{R}(x) \gamma^{\mu} \tau_{a} \psi_{R}(x), \\
& j_{L, a}^{\mu}=\bar{\psi}_{L}(x) \gamma^{\mu} \tau_{a} \psi_{L}(x) .
\end{aligned}
$$

Los correspondientes operadores de carga se obtienen integrando la componente $\mu=0$ de la corriente en las coordenadas espaciales,

$$
Q_{a}^{R}=\int d^{3} x \psi_{R}^{+}(x) \tau_{a} \psi_{R}(x), \quad Q_{a}^{L}=\int d^{3} x \psi_{L}^{+}(x) \tau_{a} \psi_{L}(x) .
$$

Al ser operadores de simetría, $Q_{a}^{R}$ y $Q_{a}^{L}$ conmutan con el hamiltoniano, por lo cual existe una base de autoestados con número de ocupación de estados de quiralidad bien definida. Los operadores de carga satisfacen las mismas relaciones de conmutación que los generadores del grupo y actúan como generadores de las transformaciones quirales. Alternativamente, podemos escribir

$$
V_{a}^{\mu}=j_{R, a}^{\mu}+j_{L, a}^{\mu}=\bar{\psi}(x) \gamma^{\mu} \tau_{a} \psi(x), \quad A_{a}^{\mu}=j_{R, a}^{\mu}-j_{L, a}^{\mu}=\bar{\psi}(x) \gamma^{\mu} \gamma_{5} \tau_{a} \psi(x),
$$

donde $V_{a}^{\mu}$ y $A_{a}^{\mu}$ son corrientes vectoriales y vectoriales axiales conservadas $\left(\partial_{\mu} V_{a}^{\mu}=\partial_{\mu} A_{a}^{\mu}=\right.$ $0)$. Entonces, la simetría quiral $\mathrm{U}(2)_{L} \otimes \mathrm{U}(2)_{R}$ es equivalente a la invarianza ante el grupo $\mathrm{U}(2)_{V} \otimes \mathrm{U}(2)_{A}$ con transformaciones en el espacio de sabor

$$
U(2)_{V}: \psi \rightarrow \exp \left(i \theta_{a}^{V} \tau_{a}\right) \psi, \quad U(2)_{A}: \psi \rightarrow \exp \left(i \theta_{a}^{A} \gamma_{5} \tau_{a}\right) \psi .
$$

Por otro lado, si consideramos una matriz arbitraria perteneciente a alguna de las transformaciones de (2.12) notamos que, como el generador $\tau_{0}$ conmuta con los restantes generadores, podemos escribir

$$
\exp \left(i \theta_{a} \tau_{a}\right)=\exp \left(i \theta_{0} \tau_{0}\right) \exp \left(i \theta_{k} \tau_{k}\right)
$$

Dado que los generadores $\tau_{k}$ son de traza nula, el segundo factor del lado derecho de la ecuación (2.17) tiene determinante 1 y pertenece al grupo $\mathrm{SU}(2)$, mientras que el factor asociado a $a=0$ corresponde a $\mathrm{U}(1)$ y es proporcional a la identidad, por lo tanto multiplica a los estados $u$ y $d$ por la misma fase. Como el caso de las transformaciones (2.15) es análogo, el lagrangiano resulta invariante ante

$$
U(2)_{R} \otimes U(2)_{L}=U(2)_{V} \otimes U(2)_{A}=U(1)_{V} \otimes S U(2)_{V} \otimes U(1)_{A} \otimes S U(2)_{A} .
$$

El análisis anterior de las simetrías no es válido cuando las partículas tienen masa no nula y se incorpora el término de masa $\mathcal{L}_{m}=-\bar{\psi} \hat{m} \psi$. Las cuadridivergencias de las corrientes dejan, en general, de ser cero, y se rompe la simetría axial U(1) ${ }_{A}$ explícitamente. 
Se pasa a obtener:

$$
\begin{aligned}
\partial_{\mu} V_{a}^{\mu} & =2 i \bar{\psi}(x)\left[\hat{m}, \tau_{a}\right] \psi(x) \\
\partial_{\mu} A_{a}^{\mu} & =2 i \bar{\psi}\left\{\hat{m}, \tau_{a}\right\} \gamma_{5} \psi(x) .
\end{aligned}
$$

En la aproximación $m_{u}=m_{d} \equiv m_{0} \neq 0$ tenemos que $\hat{m}=\operatorname{diag}\left(m_{u}, m_{d}\right)=m_{0} \mathbb{1}_{2 \times 2}$ lo cual implica la conservación de la corriente vectorial. El lagrangiano toma la forma

$$
\mathcal{L}_{m}=-m_{0} \bar{\psi} \psi=-m_{0}\left(\bar{\psi}_{L} \psi_{R}+\bar{\psi}_{R} \psi_{L}\right)
$$

y por lo tanto los subespacios left y right quedan acoplados mediante el término de masas, con lo cual no pueden realizarse sobre ellos transformaciones independientes que sean simétricas. Sin embargo, este término continúa siendo simétrico ante una transformación conjunta en la cual los estado left y right son rotados en el mismo ángulo, correspondiente al grupo $\mathrm{SU}(2)_{V}$, como puede observarse en (2.19) donde el comutador se anula. Es decir que la introducción del término de masa reduce la simetría de la siguiente manera

$$
S U(2)_{V} \otimes S U(2)_{A} \rightarrow S U(2)_{V}
$$

manteniéndose conservada únicamente la corriente vectorial (simetría de isospín). Para la corriente axial, la ruptura de simetría es pequeña en virtud de las masas corrientes pequeñas y, por supuesto, la conservación de esta corriente se recupera tomando el límite quiral. Es importante notar que tomando $m_{u} \neq m_{d}$ se dará lugar a una ruptura explícita del grupo $\mathrm{SU}(2)_{V}$ también. Las masas de los quarks $u$ y $d$ suelen tomarse iguales a pesar de que existen estimaciones según las cuales estas cantidades son levemente diferentes. En este trabajo tomaremos siempre $m_{u}=m_{d}$, aunque veremos más adelante que la interacción con un campo magnético externo rompe la simetría $\mathrm{SU}(2)_{V}$ de manera explícita.

Finalmente, las matrices de transformación de los grupos $\mathrm{U}(1)_{V}$ y $\mathrm{U}(1)_{A}$ que podemos deducir realizando el mismo procedimiento a partir de las transformaciones presentes en (2.11) están dadas por

$$
U(1)_{V}=\exp (i \theta), \quad U(1)_{A}=\exp \left(i \gamma_{5} \theta\right) .
$$

La primera de éstas está asociada a la conservación del número bariónico, mientras que la segunda, si bien es una simetría del lagrangiano clásico quiral, no se mantiene al cuantizar la teoría, dado que la medida de integración en la integral de camino correspondiente no es invariante ante dicha transformación. Este fenómeno es conocido como anomalía axial.

\subsection{Ruptura espontánea de simetrías}

Gran parte de la física se altas energías del siglo pasado se construyó sobre los principios de simetría, formulando las teorías de las interacciones fundamentales a partir de 
proponer lagrangianos invariantes ante ciertos grupos de transformaciones que respetasen las simetrías internas del sistema. Fue alentador cuando en 1960 inspeccionando el espectro de partículas elementales se descubrió que existen más simetrías internas de las que se creía. Podemos encontrar simetrías exactas y aproximadas, y también simetrías que se "rompen espontáneamente", en el sentido de que no se comportan como transformaciones de simetría de los estados físicos de la teoría, y en particular, no dejan el estado de vacío invariante.

Cuando se considera una simetría exacta del lagrangiano, como ocurre en el límite quiral de QCD, existen distintas realizaciones posibles en lo que respecta al espectro de estados. En el modo de Wigner-Weyl, la simetría del estado de vacío es la misma que la del lagrangiano. En este caso, el vacío no se encuentra degenerado y de hecho transforma de acuerdo a la representación trivial del grupo, por lo que es aniquilado por los operadores de carga asociados a la simetría. Por otro lado, en la realización de Nambu-Goldstone, el estado fundamental se ve modificado por las interacciones, de modo que ocurre una ruptura espontánea de la simetría y ésta se mantiene ante a lo sumo un subconjunto de los operadores del grupo original. Los restantes generadores ya no aniquilan el vacío, sino que crean excitaciones no masivas que se encuentran degeneradas con el fundamental, y limitan las interacciones de dichas excitaciones a bajas energías. Estas partículas, conocidas como bosones de Goldstone (o bosones de Nambu-Goldstone), se encontraron por primera vez en modelos de Godstone y Nambu, y pruebas generales de su existencia teórica fueron luego dadas por Goldstone, Salam y Weinberg [52, 53]. Por otro lado, las rupturas espontáneas de simetrías aproximadas implican la presencia de partículas de espín cero y masa pequeña, comúnmente llamadas pseudo-bosones de Goldstone. Este tipo de procesos son importantes en la teoría de interacciones fuertes, como también en áreas de física de materia condensada.

Un ejemplo clásico de una ruptura de simetría en la física de partículas elementales es la simetría quiral aproximada que describimos en la sección anterior. De acuerdo con los conocimientos actuales, esta simetría surge porque hay dos campos de quarks, up y down, que tienen masas relativamente pequeñas, pero no iguales a cero, y por lo tanto la simetría $\mathrm{SU}(2)_{A}$ no es exacta. La ruptura espontánea de esta simetría quiral aproximada implica la existencia de pseudo-bosones de Goldstone de baja masa con los mismos números cuánticos de los generadores de la simetría rota: deben ser estados de paridad negativa, espín cero, isospín igual a 1, número bariónico cero y extrañeza cero. Justamente ocurre que los más livianos de todos los hadrones son los piones, que tienen precisamente estos números cuánticos, por lo que se los identifica como los pseudo-bosones asociados con la ruptura espontánea de la simetría quiral aproximada.

Si la simetría quiral fuera exacta a nivel del lagrangiano (límite quiral) los piones no 
tendrían masa. Su pequeña pero finita masa refleja la ruptura explícita de la simetría a través de las masas de los quarks $u$ y $d$. De una forma análoga, el octete de mesones pseudoescalares corresponde a los bosones de Goldstone (ocho en este caso) asociados con la ruptura espontánea de la simetría $\mathrm{SU}(3)_{A}$ (extensión de la simetría quiral para el caso de tres sabores de quarks) [54].

Los bosones de Goldstone obedecen varios teoremas que proveen la base para la "teoría de perturbaciones quirales" (Chiral perturbation theory, $\chi \mathrm{PT}$ [55]). A diferencia de una teoría de perturbaciones en las constantes de acoplamiento, $\chi \mathrm{PT}$ corresponde a un desarrollo en serie de potencias de la masa de los quarks y el momento de los mesones, y puede ser aplicada en regiones donde la constante de acoplamiento de QCD, $\alpha_{s}$, no permite un tratamiento perturbativo.

\subsection{QCD}

La cromodinámica cuántica es una teoría de gauge de Yang-Mills invariante ante las transformaciones del grupo de simetría $\mathrm{SU}(3)_{c}$ llamado de color, donde 3 es el número de "colores". Esta teoría describe las interacciones fuertes y tiene a los quarks y gluones como los grados de libertad básicos. QCD se formula en términos de campos de fermiones que representan a los quarks y campos de gauge para los gluones. Ambos tipos de partículas llevan carga de color, la cual es responsable de esta interacción.

La teoría se define por un lagrangiano

$$
\mathcal{L}_{Q C D}=\bar{\psi}\left(i \gamma^{\mu} D_{\mu}-\hat{m}\right) \psi-\frac{1}{4} G_{a}^{\mu v} G_{\mu v}^{a}
$$

donde $D_{\mu}$ es la derivada covariante

$$
D_{\mu}=\partial_{\mu}-i g \frac{\lambda^{a}}{2} G_{\mu}^{a}
$$

y $G_{\mu \nu}^{a}$ es el tensor de fuerza del campo de los gluones

$$
G_{\mu \nu}^{a}=\partial_{\mu} G_{v}^{a}-\partial_{\nu} G_{\mu}^{a}+g f^{a b c} G_{\mu}^{b} G_{v}^{c} .
$$

El campo $\psi$ corresponde a los quarks, e incluye grados de libertad internos de color y de sabor. En la teoría completa de QCD los estados de color son 3 y se denominan red $(r)$, green $(g)$, y blue $(b)$, mientras que los estados de sabor son 6 y se definen como up $(u)$, down (d), strange (s), charm (c), bottom (b), y top ( $t$ ). Estos estados se introducen mediante un producto tensorial resultando en 18 espinores. La matriz de masas en el espacio de sabor está dada por $\hat{m}=\operatorname{diag}\left(m_{u}, m_{d}, m_{s}, m_{c}, m_{b}, m_{t}\right)$, mientras que $g$ es la constante de acoplamiento de QCD, $G_{\mu}^{a}$ es el conjunto de campos vectoriales no masivos asociados a 
los gluones con $a=1, \cdots, 8, \lambda^{a}$ es el conjunto de matrices de Gell-Mann, generadoras del álgebra del grupo SU(3), y $f^{a b c}$ indican las constantes de estructura completamente antisimétricas del grupo, que satisfacen las relaciones

$$
\begin{aligned}
{\left[\lambda^{a}, \lambda^{b}\right] } & =2 i f^{a b c} \lambda^{c}, \\
\operatorname{tr}\left(\lambda^{a} \lambda^{b}\right) & =2 \delta^{a b} .
\end{aligned}
$$

Los campos de quarks transforman de acuerdo a la representación fundamental de este grupo, la cual es de dimensión 3. En dicha representación, los generadores son las matrices de Gell-Mann, en términos de las cuales podemos escribir una transformación arbitraria del grupo de la siguiente manera

$$
U=\exp \left(i \lambda_{a} \theta^{a}\right)
$$

donde $\theta^{a}$ son los correspondientes parámetros asociados a los generadores. Ahora bien, el lagrangiano completo de QCD se construye partiendo del lagrangiano de Dirac y requiriendo que el mismo sea invariante ante transformaciones locales de $\mathrm{SU}(3)_{c}$ en lugar de globales. Es decir, en cada punto del espacio se efectúa una transformación independiente, convirtiéndose los parámetros $\theta^{a}$ en funciones de las coordenadas, como hemos discutido en la Sec. 2.1.2. Igual que en el caso de un grupo abeliano, es necesario definir en la teoría una derivada covariante a través de la cual introducimos el conjunto de campos que transforman adecuadamente de modo de cancelar los términos adicionales que se producen. En este caso se debe incorporar un campo por cada generador del grupo de simetría, resultando en los 8 campos vectoriales identificados con los gluones. De esta forma, también se introduce el acoplamiento entre el sector de gluones y el de quarks. Para que el lagrangiano resulte invariante los campos de gauge $G_{\mu}^{a}$ deben transformar conjuntamente de acuerdo a

$$
G_{\mu}^{a} \rightarrow G_{\mu}^{a}-\frac{1}{g} \partial_{\mu} \theta^{a}-f^{a b c} \theta^{b} G_{\mu}^{c}
$$

Finalmente, el término en (2.24) que involucra al tensor de fuerza del campo de los gluones de la ecuación (2.26) describe la dinámica propia de los campos de gluones en ausencia de quarks. Las propiedades de transformación de los campos $G_{\mu}^{a}$ ante el grupo SU(3) $)_{c}$ local conducen a que deba agregarse un término adicional en el tensor, en comparación con el caso abeliano, para mantener la invarianza del lagrangiano. En efecto, éste contiene, además del término de propagación, el término $f^{a b c} G_{\mu}^{b} G_{v}^{c}$ que generará la aparición de acoplamientos entre los propios gluones a través de vértices de tres y cuatro campos.

Una de las principales consecuencias de la presencia de los términos de autointeracción es el comportamiento de la constante de acoplamiento en función del impulso transferido $Q$. Es sabido que al estudiar un proceso cualquiera en una teoría de gauge renormalizable, 
la inclusión de sucesivos diagramas de Feynman que se presentan en un desarrollo en serie puede reestructurarse de modo de expresar el resultado final en términos de una constante de acoplamiento "vestida" que depende de Q. Como consecuencia, la interacción tendrá comportamientos distintos según la escala de energía involucrada en el proceso estudiado, y su dependencia funcional exacta estará determinada por los detalles de las interacciones en consideración. En el caso particular de QCD, la constante de acoplamiento tiende logarítmicamente a cero cuando el impulso transferido tiende a infinito. Esta propiedad, conocida con el nombre de libertad asintótica, permite estudiar procesos a altas energías mediante teoría de perturbaciones. Por otro lado, para impulsos transferidos pequeños, y en particular por debajo del parámetro de escala de la teoría llamado $\Lambda_{Q C D}$, la constante de acoplamiento es mayor que uno, por lo cual no es adecuada como parámetro de expansión en un desarrollo en serie. Ahora bien, dado que las escalas de energía de ligadura de los hadrones se encuentran por debajo de este umbral, el estudio de la mayoría de las propiedades hadrónicas debe tratarse mediante métodos alternativos de naturaleza no perturbativa. El propio mecanismo de confinamiento, que hace referencia al hecho empírico de que los objetos con color no existen como grados de libertad asintóticos, y para el cual no existe aún una explicación analítica satisfactoria, es una de estas propiedades. Entre los métodos a los que se puede recurrir para el estudio de la cromodinámica cuántica a bajas energías se encuentra el desarrollo de modelos efectivos de quarks, como los que se tratarán en este trabajo en los capítulos siguientes.

Una técnica alternativa que ha sido desarrollada para tratar la dinámica de QCD a bajas energías es la que se conoce como "Lattice QCD" (LQCD) o QCD en la red. Ésta consiste en resolver las ecuaciones de QCD numéricamente en forma explícita en un espacio-tiempo discretizado. En este contexto la función de partición se puede estimar utilizando el método Monte Carlo, es decir, aproximando la integral funcional correspondiente como una suma sobre configuraciones que están pesadas por la acción. El método de muestreo por importancia permite reducir los cálculos a un número limitado de configuraciones, según la probabilidad con la que ellas pueden ocurrir, $e^{-S_{E}}$, lo cual implica que este método tiene sentido si la acción euclídea es una cantidad definida positiva.

Sin embargo, la técnica de LQCD presenta algunas dificultades importantes. Una de ellas tiene que ver con que al discretizar la teoría, la cantidad de puntos en la grilla usada no puede ser demasiado alta puesto que el problema se vuelve intratable numéricamente. Por otro lado, todavía en muchos casos resulta dificultoso hacer cálculos confiables usando las masas de los quarks livianos $u$ y $d$, con lo cual se debe trabajar con valores por encima de los considerados "realistas" para luego extraer conclusiones basados en la extrapolación, complementando esto con el uso de modelos efectivos. Una limitación más fundamental aparece al intentar estudiar LQCD a densidades finitas, debido al hecho de que al agregar 
el potencial químico $\mu$ la acción euclídea resulta en general una cantidad compleja, por lo que las funciones de peso usadas para el muestreo estadístico se vuelven imaginarias y no hay manera de comparar las probabilidades asociadas a distintas configuraciones. Este es el llamado "problema del signo", que impide realizar cálculos para valores grandes de $\mu / T$, donde $T$ es la temperatura. Se han desarrollado varios métodos para tratar de superar esta complicación, pero la región de altas densidades y temperaturas bajas como la que puede esperarse en algunos núcleos estelares aún está lejos de poder ser analizada con este tipo de técnicas.

Es importante tener en cuenta que, como consecuencia del fenómeno de confinamiento en la fase de vacío, las masas de los quarks no son observables físicos. Por esta razón, cuando las masas corrientes de los quarks son usadas como parámetros de entrada de la teoría, sus valores están sujetos a un margen de variabilidad debido a que dependen de la escala de renormalización considerada. La determinación de las masas corrientes de los quarks con cierto grado de precisión puede, de hecho, realizarse mediante modelos efectivos resultando en cantidades dependientes de la escala. A partir de éstos, se concluye que los quarks de sabores $u, d$, y s son relativamente livianos, encontrándose sus masas por debajo de $\Lambda_{Q C D}$.

El descubrimiento en 1973 de la libertad asintótica en teorías de gauge no abelianas como QCD realizado por Gross, Wilczec y Politzer [56,57] persuadió a los físicos teóricos de que QCD es la teoría correcta para las interacciones fuertes. Este cálculo explicaba los resultados controversiales de un famoso experimento realizado en 1968 en SLAC (Stanford, EE.UU.) sobre dispersión inelástica entre electrones y nucleones, donde las interacciones fuertes parecían hacerse más débiles a energías altas. Pero la importancia histórica del descubrimiento de la libertad asintótica en teorías de Yang-Mills no se debe únicamente a que explica un viejo resultado experimental, sino a que por primera vez abrió la perspectiva de realizar cálculos perturbativos confiables de procesos de interacción fuerte, al menos a altas energías. La disminución de la constante de acoplamiento fuerte a altas energías, o distancias cortas, naturalmente implica que ésta aumenta a bajas energías o distancias largas, y se creyó que esto explicaría por qué no se detectan quarks o gluones libres. De acuerdo con esta hipótesis, sólo partículas neutras de color como los bariones o mesones pueden aparecer en forma aislada. Esto continúa siendo una hipótesis más que un teorema, pero hay pocas dudas de su veracidad. Aunque los quarks y gluones no pueden materializarse como partículas libres, pueden ser observadas indirectamente ya que dan origen a jets hadrónicos en procesos de colisión a altas energías.

Por otra parte, una característica importante del lagrangiano de QCD es su aproximada simetría quiral, es decir, su simetría ante transformaciones globales $\mathrm{SU}\left(\mathrm{N}_{f}\right)_{L} \otimes \mathrm{SU}\left(\mathrm{N}_{f}\right)_{R}$. Como explicamos anteriormente, esta simetría sería exacta en el límite correspondiente a 
una teoría de $N_{f}$ sabores sin masa. Para el caso de la simetría $\mathrm{SU}\left(\mathrm{N}_{f}\right)_{V}$ es suficiente con tener $N_{f}$ sabores degenerados. Si bien en QCD todos los quarks tienen masas no nulas, la simetría quiral es un concepto útil en el sector de los quarks $u$ y $d\left(N_{f}=2\right)$ e incluso, aunque con menor exactitud, cuando el quark $s$ es incluido también $\left(N_{f}=3\right)$. Esta característica se debe a que las masas corrientes de los quarks $u$ y $d$ se encuentran en el orden de unos pocos $\mathrm{MeVs}$ y son aproximadamente iguales, por lo cual es de esperar en principio que la simetría quiral $\mathrm{SU}(2)_{A} \otimes \mathrm{SU}(2)_{V}$ se encuentre aproximadamente conservada. La $\mathrm{SU}(2)_{V}$ es, en efecto, una simetría aproximada del vacío de QCD, reflejada por la existencia de multipletes de isospín casi degenerados en el espectro hadrónico, y la existencia de corrientes conservadas aproximadas en procesos dominados por la interacción fuerte.

A su vez, la evidencia experimental y teórica indica que la simetría $\mathrm{SU}(2)_{A}$ se realiza en el modo de Goldstone. Dado que el operador de carga $Q_{A}$ cambia de paridad de los estados, la realización de esta simetría en el modo de Wigner-Weyl implicaría la existencia de un "compañero quiral" para cada partícula hadrónica, es decir, por cada multiplete de isospín la existencia de otro multiplete degenerado con paridad opuesta, lo cual no es observado en la naturaleza. Además, las masas hadrónicas se encuentran muy por encima de las masas corrientes de los quarks livianos, sugiriendo la existencia de un mecanismo dinámico de generación de masa, lo cual a su vez genera la ruptura de la simetría. Estas observaciones sugieren que una formulación adecuada de QCD puede obtenerse partiendo de un lagrangiano aproximadamente simétrico ante $\mathrm{SU}(2)_{A} \otimes \mathrm{SU}(2)_{V}$, donde la simetría axial se rompe en forma dinámica. En este marco, los operadores $Q_{A}$ de la simetría rota crean un triplete de isospín de bosones de Goldstone, que pueden identificarse con los piones. Además, dado que los mismos son no masivos, el hecho de que los componentes del triplete piónico tengan masa excepcionalmente baja en relación a otros hadrones $\left(m_{\pi} / m_{N} \simeq 0,15\right)$ sostiene la idea de que los piones son bosones de Goldstone, cuya masa no nula se origina en la leve ruptura explícita de simetría producida por las masas corrientes.

La ruptura de simetría del estado de vacío de QCD y la subsiguiente generación de masa dinámica se encuentran estrechamente relacionadas con la existencia de condensados no nulos formados a partir de productos bilineales de campos de quarks y de gluones. Entre ellos se encuentra el condensado quiral, definido como el valor de expectación en el vacío $\langle\bar{\psi} \psi\rangle$, que puede expresarse como

$$
\langle\bar{\psi} \psi\rangle=-i \int \frac{d^{4} p}{(2 \pi)^{4}} \operatorname{Tr} S(p),
$$

donde $S(p)$ es el propagador de quarks del lagrangiano completo de QCD, y la traza se toma sobre los espacios de color, sabor y Dirac. El operador $\bar{\psi} \psi$ no es invariante ante transformaciones $\mathrm{SU}(2)_{A}$, por lo cual un valor medio no nulo del mismo indica que el vacío de 
QCD se realiza en el modo de Goldstone de dicha simetría. De este modo, el condensado quiral suele considerarse como un parámetro de orden para la ruptura espontánea de la simetría quiral. Las estimaciones teóricas presentes para el condensado sugieren que esto es efectivamente así. Por ejemplo, según cálculos basados en el álgebra de corrientes y las reglas de suma de QCD se tiene que $|\langle\bar{f} f\rangle|^{1 / 3}=190-260 \mathrm{MeV}$, donde $f$ representa al sabor $u$ o al $d$. Mientras tanto, cálculos típicos en LQCD conducen a $|\langle\bar{f} f\rangle|^{1 / 3}=231 \pm 8 \pm 6 \mathrm{MeV}$. En una teoría efectiva formulada en términos de grados de libertad de quarks, también pueden ser relevantes otros condensados asociados a operadores bilineales más generales, de la forma $\langle\bar{\psi} \mathcal{O} \psi\rangle$. En particular, el valor medio no nulo de $\mathcal{O}=i \vec{\tau} \gamma_{5}$ puede relacionarse con la presencia de condensados de piones. 


\section{Capítulo 3}

\section{Modelo de Nambu-Jona-Lasinio}

En este capítulo presentaremos las características más importante del modelo de Nambu Jona-Lasinio (NJL) local y de sus extensiones con interacciones no locales. Trabajaremos para ello en el marco de la simetría $\mathrm{SU}(2)$ de sabor, considerando interacciones de tipo escalar y pseudoescalar.

\subsection{Introducción al modelo NJL}

Si queremos partir de un lagrangiano fermiónico efectivo más simple que el de QCD pero con características y simetrías similares, nos vemos naturalmente inducidos a considerar el lagrangiano utilizado por Y. Nambu y G. Jona-Lasinio en sus trabajos de 1961 [24, 25]. Como comentamos en el Cap. 1 este modelo fue originalmente concebido para describir nucleones interactuantes, por lo que es necesario reinterpretar los grados de libertad fermiónicos en términos de quarks, suponiendo que las interacciones locales de dos cuerpos se corresponden con una interacción efectiva, resultante de complicados procesos gluónicos.

La principal utilidad de este modelo radica en que puede ser diseñado para incorporar las simetrías globales de QCD, y permite estudiar en detalle el mecanismo de ruptura espontánea de la simetría quiral y sus manifestaciones en la física de hadrones, como la generación dinámica de masa de los quarks, la aparición de un condensado de quarks y el papel de los piones como bosones de Goldstone. Tal vez el punto débil más importante del modelo es que no posee la propiedad de confinamiento de color de QCD. Su aplicabilidad está entonces limitada a aquellos fenómenos hadrónicos y nucleares que no dependen sensiblemente de los detalles del mecanismo de confinamiento. No obstante, cabe esperar que para muchos aspectos importantes de la física hadrónica de bajas energías, las simetrías de QCD sean tan o más importantes que el confinamiento.

Para comenzar entonces analizaremos el modelo NJL más simple, basado en el siguien- 
te lagrangiano de dos sabores:

$$
\mathcal{L}_{\mathrm{NJL}}=\bar{\psi}\left(i \gamma^{\mu} \partial_{\mu}-\hat{m}\right) \psi+\mathcal{L}_{\text {int }}
$$

donde la matriz de masas de quarks desnudas es $\hat{m}=\operatorname{diag}\left(m_{u}, m_{d}\right)$, y el lagrangiano de interacción $\mathcal{L}_{\text {int }}$ viene dado por un acoplamiento local de cuatro fermiones. Esta interacción debe satisfacer la simetría quiral $\mathrm{SU}(2)_{R} \otimes \mathrm{SU}(2)_{L}$ junto con la simetría de color $\mathrm{SU}(3)_{c}$ y las simetrías discretas C, P y T.

A partir de la propuesta original de Nambu y Jona-Lasinio, consideraremos un lagrangiano de interacción de la forma

$$
\mathcal{L}_{\text {int }}=G\left[(\bar{\psi} \psi)^{2}+\left(\bar{\psi} i \gamma_{5} \vec{\tau} \psi\right)^{2}\right]
$$

De este modo, en este modelo se considera a los quarks livianos $u$ y $d$ como los grados de libertad del sistema, suponiendo que en el límite de bajas energías los grados de libertad gluónicos están absorbidos dentro de una interacción efectiva local entre quarks.

\subsubsection{Generación dinámica de la masa fermiónica}

Consideremos el lagrangiano de interacción $\mathcal{L}_{\text {int }}$, con un número de fermiones $N_{f}=2$ de igual masa $m$. Si la constante de acoplamiento $G$ es pequeña se puede aplicar la teoría de perturbaciones para trabajar con los términos de interacción. Sin embargo, si el acoplamiento es fuerte es preciso realizar resumaciones. En este último caso comúnmente se utiliza la aproximación de Hartree (campo medio), en la que se toman en cuenta sólo los términos directos. Esto equivale a linealizar la interacción en la ecuación (3.2) reemplazando los términos $(\bar{\psi} \Gamma \psi)^{2}$ por $2 \bar{\psi} \Gamma \psi\langle\bar{\psi} \Gamma \psi\rangle$, donde $\langle\bar{\psi} \Gamma \psi\rangle$ es el valor de expectación en el vacío (estado fundamental) de $\bar{\psi} \Gamma \psi$, y $\Gamma$ es cualquiera de los operadores que aparecen en $\mathcal{L}_{\text {int }}$. Como el vacío debe ser invariante de Lorentz y debe conservar paridad, el único valor de expectación no nulo en el nivel fundamental es $\langle\bar{\psi} \psi\rangle$, el condensado quark-antiquark relacionado con la densidad escalar $\bar{\psi} \psi=\psi^{\dagger} \gamma_{0} \psi$. Entonces, la ecuación de Dirac en la aproximación de campo medio resulta

$$
\left[i \gamma_{\mu} \partial^{\mu}-\hat{m}+2 G\langle\bar{\psi} \psi\rangle\right] \psi(x)=0
$$

y es posible definir una masa fermiónica dinámica

$$
M=m-2 G\langle\bar{\psi} \psi\rangle,
$$

con $\langle\bar{\psi} \psi\rangle=\langle\bar{u} u+\bar{d} d\rangle$, generada por una interacción escalar suficientemente fuerte del fermión con el vacío de Dirac. A la ecuación (3.4) se la denomina ecuación del gap del modelo NJL, en analogía con la correspondiente ecuación que determina el gap de energía en 


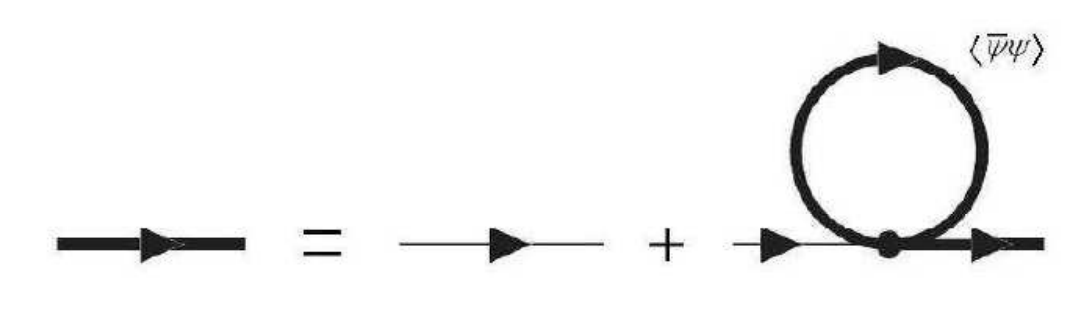

Figura 3.1: Representación diagramática de la ecuación del gap (3.4). Las líneas fina y gruesa representan el propagador del quark desnudo y vestido respectivamente. El loop fermiónico representa al condensado quiral.

un superconductor. Dicha ecuación se puede representar diagramáticamente mediante la Figura 3.1.

En la aproximación de campo medio el condensado $\langle\bar{\psi} \psi\rangle$ está dado por

$$
\langle\bar{\psi} \psi\rangle=-i \operatorname{Tr} S_{F}(0),
$$

donde $S_{F}$ es el propagador de Dirac definido por

$$
S_{F}(x-y)=-i\langle T[\psi(x) \bar{\psi}(-x)]\rangle=\int \frac{d^{4} p}{(2 \pi)^{4}} \frac{e^{i p \cdot(x-y)}}{\not p-M+i \epsilon} .
$$

La aparición de $S_{F}(0)$ en (3.5) refleja el loop cerrado de la Figura 3.1, con la línea fermiónica comenzando y terminando en el mismo punto del espacio-tiempo. Como la masa dinámica $M$ está presente en la expresión de $\langle\bar{\psi} \psi\rangle$, la ecuación del gap es una ecuación de Hartree autoconsistente.

La integral $S_{F}(0)$ es cuadráticamente divergente y requiere, por lo tanto, de un procedimiento de regularización apropiado. La prescripción más sencilla consiste en introducir un cut-off $\Lambda_{3}$ para el módulo del trivector momento $\vec{p}$ e integrar sobre la componente temporal $p_{0}$ en la ecuación (3.5). Se obtiene

$$
\langle\bar{\psi} \psi\rangle=-\frac{N_{c} N_{f}}{\pi^{2}} \int_{0}^{\Lambda_{3}} d p \vec{p}^{2} \frac{M}{\sqrt{\vec{p}^{2}+M^{2}}} .
$$

donde $N_{c}=3$ y $N_{f}=2$ son el número de colores y el número de sabores respectivamente. La ecuación del gap describe la interacción de un fermión con la parte "activa" del mar de Dirac, la cual corresponde a impulsos $|\vec{p}| \leqslant \Lambda_{3}$. Equivalentemente se puede interpretar el cut-off pensando que la interacción controlada por $G$ está "prendida" mientras $|\vec{p}| \leqslant \Lambda_{3}, \mathrm{y}$ se "apaga" para momentos superiores $|\vec{p}|>\Lambda_{3}$, en forma consistente con la hipótesis de libertad asintótica.

A partir de la ecuación (3.7), se reescribe la ecuación del gap como

$$
M=m+\frac{2 G N_{c} N_{f}}{\pi^{2}} \int_{0}^{\Lambda_{3}} \vec{p}^{2} d p \frac{M}{\sqrt{\vec{p}^{2}+M^{2}}} .
$$


Para el caso $m=0$ (límite quiral), es fácil ver que esta ecuación tiene una solución no trivial $(M \neq 0)$ cuando la constante de acoplamiento $G$ supera un valor crítico $G_{c r i t}=\pi^{2} / \Lambda_{3}^{2}$. Los quarks adquieren en ese caso una masa dinámica.

La generación dinámica de la masa va en paralelo con la reestructuración del vacío: para $G>G_{\text {crit }}$ el vacío contiene un condensado $\langle\bar{\psi} \psi\rangle$ no nulo. Como la densidad escalar $\bar{\psi} \psi$ rompe la simetría quiral, el estado fundamental no tiene la simetría $\mathrm{SU}(2)_{R} \otimes \mathrm{SU}(2)_{L}$ del lagrangiano. La simetría quiral se rompe espontáneamente, y el condensado $\langle\bar{\psi} \psi\rangle$ es el correspondiente parámetro de orden.

Por lo tanto, se distinguen las dos fases de la simetría quiral discutidas previamente:

1. La fase de Wigner-Weyl, en donde $\langle\bar{\psi} \psi\rangle=0$ y los fermiones no tienen masa. Equivalentemente, la carga axial $Q_{5}=\int d^{3} x \bar{\psi} \gamma_{0} \gamma_{5} \psi$ aniquila el vacío: $Q_{5}|0\rangle=0$. En el modelo NJL este es el caso para $G<G_{\text {crit }}$.

2. La fase de Nambu-Goldstone en donde $\langle\bar{\psi} \psi\rangle \neq 0$ y los fermiones adquieren masas dinámicas. En esta fase la simetría quiral está rota espontáneamente y existe un bosón de Nambu-Goldstone no masivo. La carga axial no aniquila el vacío: $Q_{5}|0\rangle \neq 0$. En el modelo NJL esto sucede cuando $G>G_{\text {crit }}$.

Para el caso de $m \neq 0$, si bien la masa dinámica nunca llega a ser cero, tiene un comportamiento similar al descrito para $m=0$, mostrando una curva asintótica a la correspondiente al límite quiral, tanto para el rango de $G$ pequeños como también para $G \Lambda_{3}^{2} \gg 1$.

\subsubsection{Bosón de Goldstone pseudoescalar}

Como ya se ha mencionado, la ruptura espontánea de una simetría global implica la existencia de modos de frecuencia cero llamados bosones de Nambu-Goldstone. En este caso se trata de modos fermión-antifermión pseudoescalares neutros $\left(J^{P}=0^{-}\right)$identificados con los piones. Su espín y paridad reflejan los números cuánticos de la simetría axial $\mathrm{SU}(2)_{A}$ rota.

Para analizar como emergen del modelo estos piones de Goldstone sin masa como consecuencia de la ruptura de simetría $\mathrm{SU}(2)_{A}$, construimos la matriz $T$ que surge de resolver la ecuación de Bethe-Salpeter (ecuación de dos cuerpos) en el canal fermión-antifermión pseudoescalar para un dado cuadrimomento cuadrado $q^{2}$ del mesón, ilustrado en la Figura 3.2:

$$
T_{\pi}\left(q^{2}\right)=K_{\pi}+K_{\pi} J_{\pi}\left(q^{2}\right) K_{\pi}+\cdots=\frac{K_{\pi}}{1-K_{\pi} J_{\pi}\left(q^{2}\right)} .
$$

La interacción en este canal está dada por el término $G\left(\bar{\psi} i \gamma_{5} \vec{\tau} \psi\right)^{2}$ del lagrangiano de la ecuación (3.2). Entonces, $K_{\pi}=2 G$, y $J_{\pi}$ es la integral a un loop de interacción dada por 


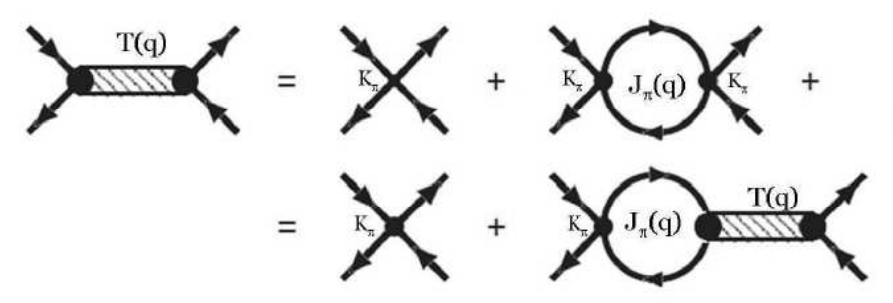

Figura 3.2: Representación esquemática de la ecuación de Bethe-Salpeter para la matriz quark-antiquark $T$. Los propagadores de mesones aparecen como bandas sombreadas, mientras que las líneas sólidas corresponden a los quarks vestidos en la aproximación de Hartree (ver Figura 3.1).

$$
J_{\pi}\left(q^{2}\right)=i \operatorname{Tr} \int \frac{d^{4} p}{(2 \pi)^{4}}\left[i \gamma_{5} \tau_{k} \frac{1}{\not p+\frac{q}{2}-M+i \epsilon} i \gamma_{5} \tau_{k} \frac{1}{\not p-\frac{\phi}{2}-M+i \epsilon}\right] .
$$

Para regularizar esta integral puede usarse un proceso idéntico al utilizado en la ecuación del gap (3.4).

La masa $m_{\pi}$ del pion queda determinada por los polos de la matriz $T$, es decir, por la condición

$$
1-2 G J_{\pi}\left(q^{2}=m_{\pi}^{2}\right)=0
$$

Utilizando la ecuación del gap (3.8) es posible ver que en el límite quiral una solución de esta ecuación es $m_{\pi}=0$, lo que significa que el pion es un pseudo-bosón de Goldstone, un modo de masa nula que surge como consecuencia de una ruptura de simetría. Para ver esto partimos de

$$
J_{\pi}\left(q^{2}=0\right)=-i \operatorname{Tr} \int \frac{d^{4} p}{(2 \pi)^{4}}\left[\gamma_{5} \tau_{k} \frac{\not p+M}{p^{2}-M^{2}+i \epsilon} \gamma_{5} \tau_{k} \frac{\not p+M}{p^{2}-M^{2}+i \epsilon}\right],
$$

y utilizando que $\operatorname{Tr}_{D}\left[\gamma_{5}(\not p+M) \gamma_{5}(\not p+M)\right]=\operatorname{Tr}_{D}\left(M^{2}-\not p \not p\right)=-4\left(p^{2}-M^{2}\right)$, se tiene

$$
J_{\pi}\left(q^{2}=0\right)=4 N_{f} N_{c} i \int \frac{d^{4} p}{(2 \pi)^{4}} \frac{1}{p^{2}-M^{2}+i \epsilon}=\frac{i}{M} \operatorname{Tr} S_{F}(0) .
$$

A partir de las ecuaciones (3.8) y (3.5) se observa que

$$
K_{\pi} J_{\pi}\left(q^{2}=0\right)=-\frac{2 G}{M}\langle\bar{\psi} \psi\rangle=1,
$$

por lo cual se verifica la validez del teorema de Nambu-Goldstone.

El próximo paso es examinar la influencia de la ruptura explícita de la simetría quiral, que se produce al considerar el término $\mathcal{L}_{m}=-m \bar{\psi} \psi$, con una masa fermiónica desnuda 
$m$ no nula. En $\mathrm{SU}(2)$ ante una rotación quiral $\psi \rightarrow \exp \left(i \gamma_{5} \tau^{a} \theta_{a} / 2\right)$, para ángulo $\theta$ pequeño, este término se transforma en

$$
\delta \mathcal{L}_{\theta}=-m \bar{\psi}\left(i \gamma_{5} \tau^{a} \theta_{a}-\frac{1}{2} \theta_{a}^{2}+\cdots\right) \psi
$$

La densidad pseudoescalar $\bar{\psi} i \gamma_{5} \psi$ es la fuente del campo de piones $\phi$, por lo que podemos identificar $\theta=\phi / f_{\pi}$, donde $f_{\pi}$ es una constante de normalización, con dimensiones de energía. Esta $f_{\pi}$ es la constante de decaimiento del pion, definida por el elemento de matriz

$$
\left\langle 0\left|\mathcal{J}_{A \mu}^{a}(0)\right| \pi^{b}(q)\right\rangle=\left\langle 0\left|\bar{\psi}(0) \gamma_{\mu} \gamma_{5} \tau^{a} \psi(0)\right| \pi^{b}(q)\right\rangle=2 i f_{\pi} q_{\mu} \delta^{a b},
$$

que describe el decaimiento débil del pion vía la corriente axial $\mathcal{J}_{A \mu}^{a}$.

Consideremos ahora el término de orden $\theta^{2}=\phi^{2} / f_{\pi}^{2}$ en la ecuación (3.15). Este término tiene la forma $m /\left(2 f_{\pi}^{2}\right) \bar{\psi} \psi \phi^{2}$. Esto corresponde exactamente al término de masa $\mathcal{L}_{m}=-\frac{1}{2} m_{\pi}^{2} \phi^{2}$ para el pion. Usando nuevamente la aproximación de campo medio con el reemplazo $\bar{\psi} \psi \rightarrow\langle\bar{\psi} \psi\rangle$, podemos hallar la relación de Gell-Mann-Oakes-Renner:

$$
m_{\pi}^{2} f_{\pi}^{2}=-m\langle\bar{\psi} \psi\rangle .
$$

Esta importante ecuación conecta los parámetros de la ruptura explícita y espontánea de la simetría quiral con las constantes $m_{\pi}$ y $f_{\pi}$ características del pion. La masa fermiónica desnuda finita $m$ eleva la masa del pion $m_{\pi}$ desde el valor nulo que tenía en el límite quiral, de tal manera que $m_{\pi}$ es proporcional a $\sqrt{m}$. Notar que el teorema de Goldstone se verifica nuevamente para $m \rightarrow 0$.

En la Sec. 2.3 se comentó que, debido al confinamiento, las masas corrientes de los quarks no son observables físicos, sino que dependen de la escala de renormalización. Es interesante mencionar que lo mismo ocurre para el condensado $\langle\bar{\psi} \psi\rangle$. En cambio, los valores de las constantes $m_{\pi}$ y $f_{\pi}$ en el lado izquierdo de la ecuación (3.17) son cantidades físicas medibles experimentalmente.

El escenario de ruptura espontánea de simetría recién descrito es de naturaleza muy general, independientemente de la forma precisa de la interacción subyacente. Existen analogías de este mecanismo en diversas áreas de la física. Uno de los ejemplos más conocidos es el de un ferromagneto: su fase de baja temperatura se caracteriza por una magnetización no nula, análoga al condensado quiral $\langle\bar{\psi} \psi\rangle$. El Hamiltoniano del sistema de espines que describe al ferromagneto es simétrico ante rotaciones espaciales. Sin embargo, en el estado fundamental la magnetización apunta en una dirección definida en el espacio: muchos espines cooperan para formar el material magnetizado macroscópicamente. Lentas variaciones en la dirección de magnetización producen un movimiento de onda larga de los espines, que corresponde al bosón de Nambu-Goldstone asociado a la simetría rotacional espontáneamente rota. 


\subsection{Extensiones no locales del modelo NJL}

Como forma de obtener un esquema que se aproxime más a la teoría de QCD, se han propuesto extensiones que incluyen interacciones no locales [58]. De hecho, la no localidad surge naturalmente en el contexto de varios enfoques bien establecidos para la dinámica de quarks a bajas energías, como por ejemplo en el caso del modelo de líquido de instatones [36] o los modelos que utilizan desarrollos de Schwinger-Dyson [37]. También los cálculos de LQCD [59] indican que las interacciones entre los quarks tienen lugar para un determinado rango en el espacio de momentos. Los modelos que incluyen interacciones no locales ofrecen la posibilidad de elegir los parámetros de modo tal de reproducir los resultados de LQCD para las dependencias con el momento de la función de masa $m(p)$ y la función de renormalización $Z(p)$ en el propagador efectivo para los quarks, así como los valores empíricos de $m_{\pi}$ y $f_{\pi}$.

Por otra parte, las extensiones no locales del modelo NJL no muestran algunos de los inconvenientes mencionados de la teoría local. En efecto, las interacciones no locales permiten regularizar el modelo de tal manera que la interacción efectiva resulte finita a todos los órdenes en el desarrollo en loops y por lo tanto no haya necesidad de introducir cut-offs adicionales [60]. Además, puede verse que las anomalías se preservan [39] y las cargas son cuantizadas correctamente.

Con el propósito de hacer el problema manejable, la mayoría de los cálculos reportados en la literatura trabajan con interacciones no locales que son separables en el espacio de momento. De hecho, básicamente fueron consideradas dos alternativas para introducir la no localidad de un modo separable. La primera de ellas esta inspirada en el líquido de instantones de QCD, mientras que en la segunda alternativa la no localidad surge de una aproximación separable para una interacción efectiva de intercambio de un gluon.

\subsubsection{Interacciones no locales}

Como fue mencionado anteriormente, un paso adelante hacia un modelo efectivo más acorde con la teoría fundamental es el sugerido por la representación de instantones del vacío de QCD [61], donde la interacción se implementa mediante un vértice efectivo, no local y separable. La naturaleza separable de la interacción permite simplificaciones en los cálculos y a la vez la no localidad provee al modelo de una regularización natural, a través de la presencia de factores de forma suaves (como funciones gaussianas o lorentzianas) que aseguran la convergencia ultravioleta de las integrales.

Alternativamente, una interacción efectiva no local entre quarks puede generarse con- 
siderando el intercambio de un gluon. Comenzamos por escribir la acción efectiva como

$$
S=\int \frac{d^{4} p_{1}}{(2 \pi)^{4}} \frac{d^{4} p_{2}}{(2 \pi)^{4}}(2 \pi)^{4} \delta^{(4)}\left(p_{1}-p_{2}\right) \bar{\psi}\left(p_{1}\right)[\not p-m] \psi\left(p_{2}\right)+S_{\text {int }} .
$$

En este esquema el término de interacción viene dado por

$$
S_{i n t}=-\frac{g^{2}}{2} \int \frac{d^{4} p_{1}}{(2 \pi)^{4}} \frac{d^{4} p_{1}^{\prime}}{(2 \pi)^{4}} \frac{d^{4} p_{2}}{(2 \pi)^{4}} \frac{d^{4} p_{2}^{\prime}}{(2 \pi)^{4}} j_{\mu}^{a}\left(p_{1}, p_{1}^{\prime}\right) D_{a b}^{\mu v} j_{v}^{b}\left(p_{2}, p_{2}^{\prime}\right),
$$

donde $g$ es la constante de acoplamiento quark-gluon y la matriz $D_{a b}^{\mu v}$ representa un propagador efectivo del gluon. Los factores $j_{\mu}^{a}$ representan corrientes de quarks del octete de color.

Los modelos basados en interacciones de intercambio de un gluon suponen que los efectos no perturbativos pueden ser tenidos en cuenta a través del propagador $D_{a b}^{\mu v}$, que usualmente se elige de manera que ajuste fenomenológicamente. El propagador efectivo del gluon provee así una forma natural de introducir la no localidad en la interacción. Por medio de transformaciones de Fierz [30, 58], la interacción en (3.18) puede expresarse introduciendo operadores que distinguen los canales escalar, pseudoescalar, vectorial y pseudovectorial.

El término de interacción efectivo tendrá en general la forma

$$
S_{\text {int }}=\frac{1}{2} \int \frac{d^{4} p_{1}}{(2 \pi)^{4}} \frac{d^{4} p_{1}^{\prime}}{(2 \pi)^{4}} \frac{d^{4} p_{2}}{(2 \pi)^{4}} \frac{d^{4} p_{2}^{\prime}}{(2 \pi)^{4}} K_{12,1^{\prime} 2^{\prime}}\left(P, p ; P^{\prime}, p^{\prime}\right) \bar{\psi}_{1}\left(p_{1}\right) \psi_{2}\left(p_{2}\right) \bar{\psi}_{2^{\prime}}\left(p_{2}^{\prime}\right) \psi_{1^{\prime}}\left(p_{1}^{\prime}\right),
$$

donde se ha utilizado la notación $P=\left(p_{1}+p_{2}\right) / 2, p=p_{1}-p_{2}$, y los índices 1,2 , 1' y $2^{\prime}$ corren sobre color, sabor y espacio de Dirac. Por supuesto, el núcleo de la interacción $K_{12,1^{\prime} 2^{\prime}}\left(P, p, P^{\prime}, p^{\prime}\right)$ debe respetar la conservación total de momentos así como las simetrías de QCD detalladas anteriormente.

Luego de realizar la transformación de Fierz se obtiene un núcleo de la forma

$$
K_{12,1^{\prime} 2^{\prime}}\left(P, p ; P^{\prime}, p\right)=-K\left(P-P^{\prime}\right) \delta^{(4)}\left(p-p^{\prime}\right) \sum_{\alpha} c_{\alpha} \Lambda_{12}^{\alpha} \Lambda_{1^{\prime} 2^{\prime}}^{\alpha}
$$

donde $\Lambda^{\alpha}$ representa matrices en los espacios de color, sabor y Dirac, y $c_{\alpha}$ son los coeficientes que surgen de las transformaciones de Fierz. En general, en los modelos más simples, sólo se retienen las componentes singuletes de color relevantes para el mecanismo de ruptura de simetría. Esto puede justificarse teniendo en cuenta que éstos son los términos dominantes en el desarrollo en potencias de $1 / N_{c}$. Es decir,

$$
\sum_{\alpha} c_{\alpha} \Lambda_{12}^{\alpha} \Lambda_{1^{\prime} 2^{\prime}}^{\alpha} \rightarrow \sum_{\alpha} \Gamma_{12}^{\alpha} \Gamma_{1^{\prime} 2^{\prime}}^{\alpha}
$$

donde $\Gamma_{\alpha}=\left(\mathbb{1}, i \gamma_{5} \tau_{a}\right)$ con $a=1,2,3$. En cuanto a la dependencia en los momentos, la función $K\left(P-P^{\prime}\right)$ lleva a ecuaciones del gap no lineales que son complicadas de resolver. 


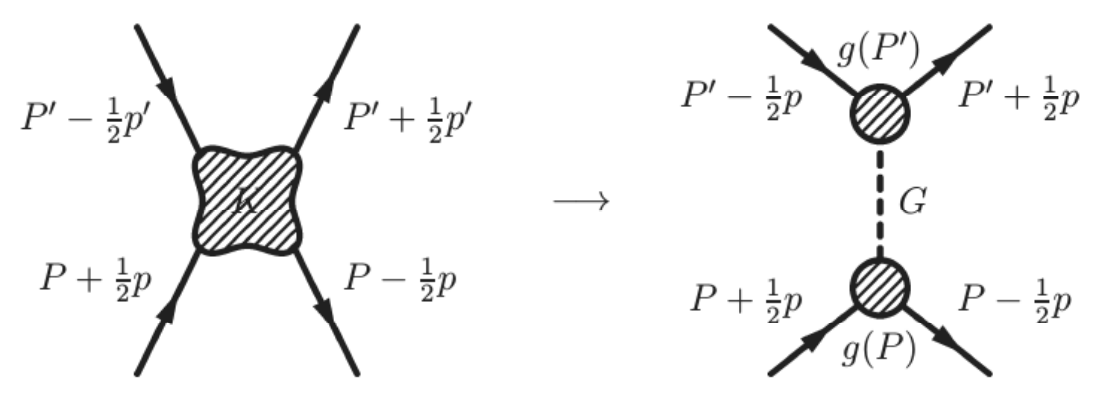

Figura 3.3: Representación diagramática de la ecuación (3.24). En el diagrama de la izquierda $K$ es un núcleo de interacción de cuatro puntos que depende de todos los momentos, mientras que en la derecha aparece en cada vértice el factor de forma $g(p)$, que depende de un solo momento.

Introduciremos aquí una simplificación adicional que consiste en suponer que la interacción es separable, es decir,

$$
K\left(P-P^{\prime}\right) \longrightarrow K_{0} g(P) g\left(P^{\prime}\right),
$$

donde $K_{0}$ es una constante y $g(0)=1$. Teniendo en cuenta las aproximaciones hasta aquí mencionadas, una forma adecuada para el núcleo de interacción es

$$
K_{12,1^{\prime} 2^{\prime}}\left(P, p ; P^{\prime}, p\right)=-\delta_{p p^{\prime}} K_{0} g(P) g\left(P^{\prime}\right) \Gamma_{12}^{\alpha} \Gamma_{1^{\prime} 2^{\prime}}^{\alpha},
$$

donde la suma sobre $\alpha=0,1,2,3$ está sobreentendida. Este reemplazo está representado en la Figura 3.3. En la función $g(P)$, que usualmente recibe el nombre de regulador o factor de forma, se encuentra la información acerca de la no localidad de la interacción.

Debido a este carácter no local de la interacción, los quarks adquieren masas dinámicas (o autoenergías) que dependen de su momento $P$ a través de los factores de forma según $\Sigma(P)=m+[\Sigma(0)-m] g(P)$. En el espacio de Minkowski, para que un quark exista en forma aislada se debe cumplir que $\Sigma^{2}(P)=P^{2}$ para valores reales de $P$. Dada la dependencia en $P$ de la autoenergía, esta ecuación no necesariamente presenta soluciones reales. En particular, dependiendo del factor de forma, puede ocurrir que el propagador del quark no tenga polos reales a partir de un valor crítico de $\Sigma(0)$, lo que puede interpretarse como una situación de confinamiento.

\subsubsection{Modelo quiral no local de quarks con renormalización de la función de onda}

De acuerdo con resultados de LQCD, podemos considerar modelos no locales adoptando una descripción más confiable del propagador efectivo de los quarks incluyendo 
en él una renormalización de función de onda (WFR) dependiente del momento [59, 62]. Estos resultados sugieren que dicha renormalización puede tener un efecto del orden del $30 \%$ o mayor a momento cero. Además, estos cálculos también muestran que las masas de los quarks tienden a sus valores asintóticos de un modo suave. Por lo tanto, es importante estudiar la incorporación de estas características en este tipo de modelos, y analizar su rol en la predicción para los diferentes observables hadrónicos.

El lagrangiano que utilizaremos en esta sección nos permitirá analizar las características de la no localidad en un modelo quiral con simetría SU(2) de sabor, así como incorporar una dependencia del propagador de quark con el momento a través de la masa y de la función de renormalización de onda. Calcularemos en este marco las propiedades del mesón escalar $\sigma$ y los mesones pseudoescalares $\pi_{a}$. El mesón $\sigma$ tiene los mismos números cuánticos que el vacío: su espín es igual a cero y su paridad es par $\left(J^{P C}=0^{++}\right)$, por ello puede condensar en el vacío y romper simetrías.

Debido a que deseamos comparar los resultados de este modelo con los obtenidos en LQCD, escribiremos la acción en el espacio euclídeo. Se tiene

$$
S_{E}=\int d^{4}(x)\left\{\bar{\psi}(x)(-i \not \partial+\hat{m}) \psi(x)-\frac{G}{2}\left[j_{S}(x) j_{S}(x)+j_{P}^{a}(x) j_{P}^{a}(x)+j_{M}(x) j_{M}(x)\right]\right\},
$$

donde $\psi(x)$ es un doblete fermiónico $\left(N_{f}=2\right), \psi=(u d)^{T}, \mathrm{y} \hat{m}=\operatorname{diag}\left(m_{u}, m_{u}\right)$ es la matriz de las masas corrientes de los quarks. El término cinético incluye el operador $\phi=\gamma_{\mu} \partial_{\mu}$, que en el espacio euclídeo se define como $\vec{\gamma} \cdot \vec{\nabla}+\gamma_{4} \frac{\partial}{\partial \tau}$ con $\gamma_{4}=i \gamma_{0}$. La no localidad del modelo es introducida a través de las corrientes $j_{S}(x), j_{P}^{a}(x)$ y $j_{M}(x)$, definidas como:

$$
\begin{aligned}
j_{S}(x) & =\int d^{4} z g(z) \bar{\psi}\left(x+\frac{z}{2}\right) \psi\left(x-\frac{z}{2}\right), \\
j_{P}^{a}(x) & =\int d^{4} z g(z) \bar{\psi}\left(x+\frac{z}{2}\right) i \gamma_{5} \tau^{a} \psi\left(x-\frac{z}{2}\right), \\
j_{M}(x) & =\frac{1}{2 \varkappa} \int d^{4} z f(z) \bar{\psi}\left(x+\frac{z}{2}\right) i \overleftrightarrow{\not} \psi\left(x-\frac{z}{2}\right),
\end{aligned}
$$

con $a=1,2,3, \mathrm{y} u\left(x^{\prime}\right) \overleftrightarrow{\partial} v(x)=u\left(x^{\prime}\right) \partial_{x} v(x)-\partial_{x} u\left(x^{\prime}\right) v(x)$

Las funciones $g(z)$ y $f(z)$ son factores de forma covariantes responsables del carácter no local de las interacciones. Para mantener la invarianza quiral el factor de forma $g(z)$ debe ser el mismo para las cuatro corrientes de quark estándar $j_{S}(x)$ y $j_{P}^{a}(x)$, y los acoplamientos correspondientes deben llevar una misma constante de acoplamiento $G$.

El término de interacción de momento es autoinvariante ante transformaciones quirales, por lo tanto presenta un factor de forma $f(z)$ diferente en general al del resto de las interacciones. Por comodidad, sin embargo, utilizaremos también la constante de acoplamiento $G$, controlando el peso relativo entre estas interacciones y las escalares y pseudoescalares a través del parámetro de masa $\varkappa$ en la ecuación (3.26). 
La corriente $j_{S}(x)$ introduce en el propagador el factor de forma $g(z)$, dando lugar a una masa dinámica para los quarks dependiente del momento, a su vez que la "corriente de momentos" $j_{P}(x)$ es responsable de introducir la renormalización de la función de onda, a través de la dependencia con el factor de forma $f(z)$. Es por ello que resulta conveniente tomar la transformada de Fourier de $g(z)$ y $f(z)$ para llevarlos al espacio de momentos. La invarianza de Lorentz implica que las transformadas $g(p)$ y $f(p)$ sólo podrán ser funciones de $p^{2}$.

Para estudiar propiedades hadrónicas es conveniente realizar una bosonización de la teoría fermiónica. Siguiendo el procedimiento descrito en el Apéndice A, partiremos de la función de partición $\mathcal{Z}=\int \mathcal{D} \psi \mathcal{D} \psi \exp \left[-S_{E}\right]$, e introduciremos campos bosónicos auxiliares: $\sigma_{1}(x), \sigma_{2}(x)$ (escalares, relacionados respectivamente con las corrientes $\left.j_{S} \mathrm{y} j_{M}\right), \mathrm{y}$ $\pi^{a}(x)$ (pseudoescalares, asociados con $j_{P}^{a}$ ), donde el índice $a$ corre desde 1 a 3 . Luego de integrar los campos fermiónicos la función de partición en el vacío puede escribirse como

$$
\mathcal{Z}=\int \mathcal{D} \sigma_{1} \mathcal{D} \sigma_{2} \mathcal{D} \vec{\pi} \exp \left[-S_{E}^{\text {bos }}\right]
$$

donde $S_{E}^{\text {bos }}$ es la acción Eulídea bosonizada. En el espacio de impulsos, ésta viene dada por

$$
\begin{aligned}
S_{E}^{\text {bos }}= & -\log \operatorname{det} A\left(p, p^{\prime}\right) \\
& +\int \frac{d^{4} p}{(2 \pi)^{4}}\left\{\frac{1}{2 G}\left[\sigma_{1}(p) \sigma_{1}(-p)+\vec{\pi}(p) \cdot \vec{\pi}(-p)+\sigma_{2}(p) \sigma_{2}(-p)\right]\right\},
\end{aligned}
$$

donde el operador $A\left(p, p^{\prime}\right)$ es

$$
\begin{aligned}
A\left(p, p^{\prime}\right)= & (2 \pi)^{4} \delta^{(4)}\left(p-p^{\prime}\right)\left(-p+m_{c}\right)+g\left(\frac{p+p^{\prime}}{2}\right)\left[\sigma_{1}\left(p^{\prime}-p\right)+i \gamma_{5} \vec{\tau} \cdot \vec{\pi}\left(p^{\prime}-p\right)\right] \\
& +f\left(\frac{p+p^{\prime}}{2}\right) \frac{p+p^{\prime}}{2 \varkappa} \sigma_{2}\left(p^{\prime}-p\right) .
\end{aligned}
$$

Sin perder generalidad, las constantes de acoplamiento se pueden elegir de forma tal que los factores de formas quedan normalizados a $f(0)=g(0)=1$.

\subsubsection{Aproximación de campo medio}

Consideraremos ahora la aproximación de campo medio (MFA). Para ello desarrollaremos los campos bosónicos alrededor de sus valores de expectación de vacío. Basándonos en las simetrías de carga, paridad y Lorentz, supondremos que los campos escalares $\sigma_{1}(x)$ y $\sigma_{2}(x)$ tienen valores medios (invariantes traslacionales) no triviales $\bar{\sigma}_{1} \mathrm{y} \varkappa \bar{\sigma}_{2}$, respectivamente, mientras que los valores de expectación de vacío de los campos bosónicos pseudoescalares son cero (vale la pena notar que $\bar{\sigma}_{2}$ es adimensional, debido a la introducción del 
parámetro $\varkappa$ ). Esto es,

$$
\begin{aligned}
\sigma_{1}(x) & =\bar{\sigma}_{1}+\delta \sigma_{1}(x), \\
\sigma_{2}(x) & =\varkappa \bar{\sigma}_{2}+\delta \sigma_{2}(x), \\
\vec{\pi}(x) & =\delta \vec{\pi}(x) .
\end{aligned}
$$

Reemplazando en la ecuación (3.29) se puede reescribir el operador como $A\left(p, p^{\prime}\right)=$ $A_{0}\left(p, p^{\prime}\right)+\delta A\left(p, p^{\prime}\right)$, donde

$$
A_{0}\left(p, p^{\prime}\right)=(2 \pi)^{4} \delta^{(4)}\left(p-p^{\prime}\right)\left\{-\left[1-\bar{\sigma}_{2} f(p)\right] p+m_{c}+\bar{\sigma}_{1} g(p)\right\} .
$$

De este modo, el término $A_{0}\left(p, p^{\prime}\right)$ depende únicamente de los factores de forma $g(p)$ y $f(p)$ y de los valores de expectación de vacío $\bar{\sigma}_{1}$ y $\bar{\sigma}_{2}$. Por otro lado, $\delta A\left(p, p^{\prime}\right)$ dependerá de las fluctuaciones de los campos mesónicos.

Dentro de esta aproximación podemos desarrollar el logaritmo del determinante fermiónico como

$$
\log \operatorname{det} A=\operatorname{Tr} \log A=\operatorname{Tr} \log A_{0}+\operatorname{Tr}\left(A_{0}^{-1} \delta A\right)-\frac{1}{2} \operatorname{Tr}\left(A_{0}^{-1} \delta A A_{0}^{-1} \delta A\right)+\ldots,
$$

donde la traza se extiende sobre los espacios de Dirac, color, sabor y momento. El operador $A_{0}^{-1}$ viene dado por

$$
A_{0}^{-1}=\frac{(2 \pi)^{4} \delta^{4}\left(p-p^{\prime}\right) z(p)}{-\not p+m(p)}
$$

donde las funciones $m(p)$ y $z(p)$-masa efectiva dependiente del momento y renormalización de función de onda- están relacionados con los factores de forma no locales y con los valores de expectación del vacío de los campos escalares por

$$
\begin{aligned}
z(p) & =\left[1-\bar{\sigma}_{2} f(p)\right]^{-1}, \\
m(p) & =z(p)\left[m_{c}+\bar{\sigma}_{1} g(p)\right] .
\end{aligned}
$$

Calcular el determinante fermiónico es, entonces, equivalente a calcular la traza del operador $\log A\left(p, p^{\prime}\right)$ en los espacios de color, sabor, Dirac e impulsos. Debido a que en este modelo no tenemos dependencias explícitas con los números cuánticos de color de los quarks, calcular la traza en ese espacio es trivial, obteniendo un factor $N_{c}=3$ correspondiente al número total de colores del modelo. En el espacio de sabor, como consideramos los quarks $u$ y $d$ bajo una simetría $\mathrm{SU}(2)$, para obtener la traza se debe tener en cuenta las matrices de Pauli que provienen de la corrientes pseudoescalares.

Al reemplazar la ecuación (3.32) en la acción efectiva bosonizada (3.28), podemos desarrollar la misma en potencias de las fluctuaciones de los campos mesónicos, obteniendo

$$
S_{E}^{\text {bos }}=S_{E}^{\mathrm{MFA}}+S_{E}^{\text {quad }}+\ldots
$$


Aquí la acción a campo medio por unidad de volumen es [63]

$$
\frac{S_{E}^{\mathrm{MFA}}}{V^{(4)}}=-2 N_{c} \int \frac{d^{4} p}{(2 \pi)^{4}} \operatorname{Tr}_{\mathrm{D}} \log \left[\mathcal{D}_{0}^{-1}(p)\right]+\frac{1}{2 G_{S}}\left(\bar{\sigma}_{1}^{2}+\varkappa^{2} \bar{\sigma}_{2}^{2}\right),
$$

donde $\operatorname{Tr}_{\mathrm{D}}$ es la traza sobre el espacio de Dirac, y el propagador efectivo de quark a campo medio $\mathcal{D}_{0}(p)$ resulta

$$
\mathcal{D}_{0}(p)=\frac{z(p)}{-p+m(p)} .
$$

Las valores de campo medio $\bar{\sigma}_{1,2}$ se pueden calcular minimizando la acción $S_{E}^{\mathrm{MFA}}$, con lo cual se obtienen las ecuaciones de Dyson-Schwinger, también conocidas como las ecuaciones del gap. Así surge el siguiente conjunto de ecuaciones de gap acopladas [63]

$$
\begin{aligned}
& \bar{\sigma}_{1}=8 N_{C} G_{S} \int \frac{d^{4} p}{(2 \pi)^{4}} g(p) \frac{z(p) m(p)}{D(p)}, \\
& \bar{\sigma}_{2}=-8 N_{C} G_{S} \int \frac{d^{4} p}{(2 \pi)^{4}} \frac{p^{2}}{\varkappa^{2}} f(p) \frac{z(p)}{D(p)},
\end{aligned}
$$

donde definimos $D(p)=p^{2}+m(p)^{2}$.

Los condensados quirales de quarks - parámetros de orden para la transición de restauración quiral - están dados por los valores de expectación del vacío $\langle\bar{q} q\rangle$, donde $q=$ $u, d$. Las expresiones correspondientes pueden obtenerse diferenciando la función de partición a campo medio $\mathcal{Z}=\exp \left[-S_{E}^{\mathrm{MFA}}\right]$ con respecto a la masa corriente del quark correspondiente. Alejados del límite quiral, esto lleva en general a integrales divergentes. Como estamos interesados en la descripción de propiedades no triviales del vacío que surgen de las interacciones fuertes, es usual regularizar estas integrales sustrayendo las contribuciones de quarks libres (ver por ejemplo [64, 65, 66, 67]). Se tiene de este modo

$$
\langle\bar{q} q\rangle=-4 N_{C} \int \frac{d^{4} p}{(2 \pi)^{4}}\left(\frac{z(p) m(p)}{D(p)}-\frac{m_{c}}{p^{2}+m_{c}^{2}}\right) .
$$

El buen comportamiento de las funciones $g(p)$ y $f(p)$ garantiza la convergencia ultravioleta de (3.38) y (3.39).

\subsubsection{Fluctuaciones cuadráticas}

Estamos interesados en la descripción de la fenomenología de los mesones, lo que requiere ir más allá de la aproximación de campo medio. En esta subsección y la siguiente derivamos las expresiones que utilizamos para calcular las cantidades fenomenológicas medibles básicas, como las masas de los mesones y la constante de decaimiento débil del pion.

En general, las masas de los mesones se pueden obtener a partir de los términos en la acción euclídea que son cuadráticos en las variaciones de los campos bosónicos. Por 
lo tanto, siguiendo el desarrollo de $S_{E}^{b o s}$ en la ecuación (3.35) a segundo orden podemos escribir $S_{E}^{\text {quad }}$ como

$$
\begin{aligned}
S_{E}^{\text {quad }}= & \frac{1}{2} \int \frac{d^{4} p}{(2 \pi)^{4}}\left\{G_{\sigma_{1}}\left(p^{2}\right) \delta \sigma_{1}(p) \delta \sigma_{1}(-p)+G_{\sigma_{2}}\left(p^{2}\right) \delta \sigma_{2}(p) \delta \sigma_{2}(-p)\right. \\
& \left.+G_{\sigma_{1} \sigma_{2}}\left(p^{2}\right)\left[\delta \sigma_{1}(p) \delta \sigma_{2}(-p)+\delta \sigma_{2}(p) \delta \sigma_{1}(-p)\right]+G_{\pi}\left(p^{2}\right) \delta \vec{\pi}(p) \cdot \delta \vec{\pi}(-p)\right\},
\end{aligned}
$$

donde las funciones $G_{M}\left(p^{2}\right), M=\sigma_{1}, \sigma_{2}, \pi$ están dadas por integrales a un loop que surgen del determinante fermiónico en la acción bosonizada. Se tiene

$$
\begin{aligned}
G_{\sigma_{1}}\left(p^{2}\right)= & \frac{1}{G_{S}}-8 N_{c} \int \frac{d^{4} q}{(2 \pi)^{4}} g^{2}(q) \frac{z\left(q^{+}\right) z\left(q^{-}\right)}{D\left(q^{+}\right) D\left(q^{-}\right)}\left[\left(q^{+} \cdot q^{-}\right)-m\left(q^{+}\right) m\left(q^{-}\right)\right] \\
G_{\pi}\left(p^{2}\right)= & \frac{1}{G_{S}}-8 N_{c} \int \frac{d^{4} q}{(2 \pi)^{4}} g^{2}(q) \frac{z\left(q^{+}\right) z\left(q^{-}\right)}{D\left(q^{+}\right) D\left(q^{-}\right)}\left[\left(q^{+} \cdot q^{-}\right)+m\left(q^{+}\right) m\left(q^{-}\right)\right] \\
G_{\sigma_{2}}\left(p^{2}\right)= & \frac{1}{G_{S}}+\frac{8 N_{c}}{\varkappa^{2}} \int \frac{d^{4} q}{(2 \pi)^{4}} q^{2} f^{2}(q) \frac{z\left(q^{+}\right) z\left(q^{-}\right)}{D\left(q^{+}\right) D\left(q^{-}\right)}\left[\left(q^{+} \cdot q^{-}\right)-m\left(q^{+}\right) m\left(q^{-}\right)\right. \\
& \left.+\frac{\left(q^{+}\right)^{2}\left(q^{-}\right)^{2}-\left(q^{+} \cdot q^{-}\right)^{2}}{2 q^{2}}\right]
\end{aligned}
$$

donde $q^{ \pm}=q \pm p / 2$

Encontramos también en $S_{E}^{\text {quad }}$ un término de mezcla $G_{\sigma_{1} \sigma_{2}}\left(p^{2}\right)$ que viene dado por

$$
G_{\sigma_{1} \sigma_{2}}\left(p^{2}\right)=-\frac{8 N_{c}}{\varkappa} \int \frac{d^{4} q}{(2 \pi)^{4}} f(q) g(q) \frac{z\left(q^{+}\right) z\left(q^{-}\right)}{D\left(q^{+}\right) D\left(q^{-}\right)} q \cdot\left[q^{+} m\left(q^{-}\right)+q^{-} m\left(q^{+}\right)\right] .
$$

Para reescribir el sector $\sigma_{1}, \sigma_{2}$ en términos de campos mesónicos desacoplados, definimos dos nuevos campos $\sigma$ y $\sigma^{\prime}$, combinaciones lineales de $\sigma_{1}$ y $\sigma_{2}$, de modo tal que

$$
\delta \sigma=\cos \theta \delta \sigma_{1}-\sin \theta \delta \sigma_{2}, \quad \delta \sigma^{\prime}=\sin \theta^{\prime} \delta \sigma_{1}+\cos \theta^{\prime} \delta \sigma_{2} .
$$

Los ángulos $\theta$ y $\theta^{\prime}$ se ajustan de manera tal que se cancelen los términos de mezcla del sector escalar a nivel cuadrático de la acción para $p^{2}=-m_{\sigma^{(\prime)}}^{2}$, donde el signo menos se debe al hecho de que la acción está dada en el espacio euclídeo.

Una vez que los términos cruzados se han eliminado, las funciones $G_{M}\left(p^{2}\right)$ representan las recíprocas de los propagadores efectivos de los mesones. Por consiguiente, las masas mesónicas pueden obtenerse resolviendo las ecuaciones $G_{M}\left(-m_{M}^{2}\right)=0$, con $M=\sigma, \sigma^{\prime}, \pi$.

Finalmente, debe llevarse a cabo una renormalización de los campos. Los campos renormalizados $\tilde{M}(p)=M(p) / Z_{M}^{1 / 2}$ se definen fijando el residuo de $G_{M}\left(p^{2}\right)$ en el polo del propagador, es decir, requiriendo que en la vecindad del polo la contribución correspondiente al lagrangiano cuadrático esté dada por

$$
\left(\mathcal{L}_{E}^{\text {quad }}\right)_{M} \simeq \frac{1}{2}\left(p^{2}+m_{M}^{2}\right) \delta \tilde{M}(p) \cdot \delta \tilde{M}(-p)
$$


De este modo se obtiene

$$
Z_{M}^{-1}=g_{M q q}^{-2}=\left.\frac{d G_{M}(p)}{d p^{2}}\right|_{p^{2}=-m_{M}^{2}} .
$$

La constante $g_{M q q}$ se puede también interpretar como una constante de acoplamiento efectiva entre el mesón $M$ y los quarks.

\subsubsection{Decaimiento débil del pion}

Por definición la constante de decaimiento débil del pion $f_{\pi}$ está dada por el elemento de matriz de la corriente axial $\mathcal{J}_{A \mu}^{a}(x)$ entre el vacío y el estado renormalizado de un pion,

$$
\left\langle 0\left|\mathcal{J}_{A \mu}^{a}(x)\right| \tilde{\vec{\pi}}^{b}(p)\right\rangle=i e^{-i p \cdot x} \delta^{a b} f_{\pi}\left(p^{2}\right) p_{\mu},
$$

evaluados en el polo del pion. Para determinar la expresión de la corriente axial, debemos realizar una transformación de gauge a la acción efectiva $S_{E}$, introduciendo campos de gauge externos. En general, para una teoría local, esto se lleva a cabo reemplazando

$$
\partial_{\mu} \longrightarrow \partial_{\mu}-i \mathcal{G}_{\mu}
$$

Sin embargo, en nuestro modelo, dada la no localidad de las interacciones, la transformación de gauge requiere la introducción de campos de gauge no sólo a través de la derivada covariante en la ecuación (3.47), sino que también se deben realizar reemplazos adicionales a través de un transporte paralelo de los campos fermiónicos en las corrientes no locales (ver Refs. [41, 58, 63, 68, 69]):

$$
\begin{aligned}
\psi(x-z / 2) & \rightarrow W_{G}(x, x-z / 2) \psi(x-z / 2), \\
\psi^{\dagger}(x+z / 2) & \rightarrow \psi^{\dagger}(x+z / 2) W_{G}(x+z / 2, x) .
\end{aligned}
$$

Aquí, $x$ y $z$ son las variables en la definición de las corrientes no locales en la ecuación (3.26), mientras que la función $W_{G}(x, y)$ está definida por

$$
W_{G}(x, y)=P \exp \left[-i \int_{x}^{y} d s^{\mu} \mathcal{G}_{\mu}(s)\right]
$$

donde $s$ recorre un camino arbitrario que conecta $x$ con $y$. En el caso de la corriente axial introducimos campos de gauge axiales $\mathcal{W}_{\mu}^{a}(x)$, tomando

$$
\mathcal{G}_{\mu}=\frac{1}{2} \gamma_{5} \vec{\tau} \cdot \overrightarrow{\mathcal{W}}_{\mu}
$$

Una vez construida la acción efectiva con su correspondiente transformación de gauge, podemos obtener la corriente axial derivando la acción con respecto al campo $\mathcal{W}_{\mu}^{a}(x)$, y evaluando esta derivada en $\overrightarrow{\mathcal{W}}_{\mu}(x)=0$. Luego, podemos identificar los elementos de 
matriz como la derivada de la expresión resultante con respecto al campo del pion renormalizado,

$$
\left\langle 0\left|\mathcal{J}_{A \mu}^{a}(x)\right| \tilde{\pi}^{b}(p)\right\rangle=\left.\frac{\delta^{2} S_{E}^{\mathrm{bos}}}{\delta \mathcal{W}_{\mu}^{a}(x) \delta \tilde{\pi}^{b}(p)}\right|_{\mathcal{W}_{\mu}^{a}=\tilde{\pi}^{b}=0}
$$
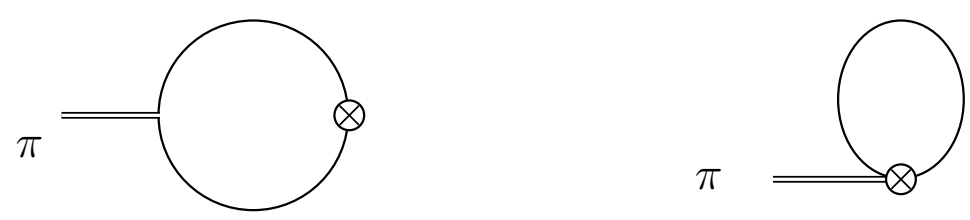

Figura 3.4: Representación diagramática de las contribuciones a la constante de decaimiento débil del pion. La cruz representa el vértice de la corriente axial.

Las contribuciones resultantes a un loop están esquematizadas en forma diagramática en la Figura 3.4. En este modelo no local se obtienen contribuciones de diagramas tipo tadpole, que no están presentes en el modelo local NJL, y surgen de la introducción de los campos de gauge a través de las ecuaciones (3.48). Finalmente obtenemos

$$
f_{\pi}=\frac{m_{c} g_{\pi q \bar{q}}}{m_{\pi}^{2}} F_{0}\left(-m_{\pi}^{2}\right)
$$

donde

$$
F_{0}\left(p^{2}\right)=8 N_{c} \int \frac{d^{4} q}{(2 \pi)^{4}} g(q) \frac{z\left(q^{+}\right) z\left(q^{-}\right)}{D\left(q^{+}\right) D\left(q^{-}\right)}\left[\left(q^{+} \cdot q^{-}\right)+m\left(q^{+}\right) m\left(q^{-}\right)\right] .
$$

Es importante destacar que este resultado no depende del camino elegido para la función de transporte en la ecuación (3.49). 


\section{Capítulo 4}

\section{Modelo NJL no local con acoplamientos vectoriales $y$ vectoriales-axiales}

En este capítulo nos concentraremos en la incorporación de interacciones vectoriales y axiales en el modelo NJL no local. Por lo tanto nuestro objetivo será construir un modelo incluyendo acoplamientos entre corrientes no locales vectoriales y vectoriales-axiales que satisfagan las simetrías de QCD, manteniendo al mismo tiempo los acoplamientos escalares y psudoescalares analizados en el capítulo anterior. De este modo, no sólo podremos calcular características propias de la fenomenología del sector vectorial, sino también podremos analizar cómo se ve modificado el sector escalar-pseudoescalar por la incorporación de estos nuevos acoplamientos.

Nos dedicaremos primero a trabajar con el formalismo de forma tal de poder derivar las expresiones analíticas para algunas propiedades básicas de los mesones vectoriales y axiales de paridad $J^{P}=1^{-}$y $J^{P}=1^{+}$respectivamente, como masas y anchos de decaimiento. Luego presentaremos los resultados numéricos obtenidos considerando formas funcionales de los factores de forma consistentes con datos obtenidos de LQCD para los propagadores efectivos de quarks.

\subsection{Construcción del modelo}

\subsubsection{Aproximación de campo medio}

Para obtener la acción euclídea efectiva se debe primero proponer un lagrangiano que contenga las simetrías que se observan en QCD. El objetivo es extender la acción propuesta en el capítulo anterior [ecuación (3.25)] añadiendo interacciones vectoriales y axiales que 
mantengan las simetrías $\mathrm{SU}(2)_{V} \otimes \mathrm{SU}(2)_{A} \otimes \mathrm{U}(1)_{V}$.

Las interacciones son del tipo $\left(\bar{\psi} \Gamma_{a} \psi\right)^{2}$ con $\Gamma_{a}=\left(\gamma_{\mu}, \gamma_{\mu} \vec{\tau}\right)$ para el caso vectorial, y $\Gamma_{a}=$ $\left(\gamma_{\mu} \gamma_{5}, \gamma_{\mu} \gamma_{5} \vec{\tau}\right)$ para el vectorial-axial. A la hora de proponer un lagrangiano es necesario conocer cómo transforman estos términos para saber si conservan las simetrías de QCD.

Es sencillo comprobar la invarianza de los acoplamientos corriente-corriente ante las transformaciones $\mathrm{SU}(2)_{V}$ y U(1) $)_{V}$. Sin embargo no es tan evidente en el caso de la simetría $\mathrm{SU}(2)_{A}$. Esto se debe a que esta transformación contiene a las matrices $\gamma_{5}$ y $\vec{\tau}$ del siguiente modo $\psi \rightarrow \exp \left(-i \vec{\tau} \cdot \vec{\theta} \gamma_{5} / 2\right) \psi=\left[\cos (\theta / 2)-i \vec{\tau} \cdot \hat{\theta} \gamma_{5} \sin (\theta / 2)\right] \psi$, y se debe tener en cuenta tanto las reglas de conmutación entre $\gamma_{5}$ y las matrices $\gamma_{\mu}$ que ahora se encuentran en las interacciones, como las reglas de conmutación de las matrices $\vec{\tau}$ que son los generadores del grupo SU(2).

Al calcular el lagrangiano trasformado se llega a que la suma $\left(\bar{\psi} \gamma_{\mu} \vec{\tau} \psi\right)^{2}+\left(\bar{\psi} \gamma_{\mu} \gamma_{5} \vec{\tau} \psi\right)^{2}$ es una combinación invariante ante las transformaciones $\mathrm{SU}(2)_{A}$ y entonces es necesario incluir ambos términos en el lagrangiano acoplados con la misma constante, a diferencia de los términos $\left(\bar{\psi} \gamma_{\mu} \psi\right)^{2}$ y $\left(\bar{\psi} \gamma_{\mu} \gamma_{5} \psi\right)^{2}$ que son invariantes independientemente.

Por lo tanto puede proponerse como lagrangiano quiral del modelo NJL local a

$$
\begin{aligned}
\mathcal{L}_{\text {quiral }}=\bar{\psi} i \not \phi \psi+G\left[(\bar{\psi} \psi)^{2}+\left(\bar{\psi} i \gamma_{5} \tau \psi\right)^{2}\right]+H\left[\left(\bar{\psi} \gamma_{\mu} \vec{\tau} \psi\right)^{2}\right. & \left.+\left(\bar{\psi} \gamma_{\mu} \gamma_{5} \vec{\tau} \psi\right)^{2}\right] \\
& +I\left(\bar{\psi} \gamma_{\mu} \psi\right)^{2}+J\left(\bar{\psi} \gamma_{\mu} \gamma_{5} \psi\right)^{2}
\end{aligned}
$$

A partir de este lagrangiano, introduciendo interacciones no locales y el término de masa, podemos obtener la acción efectiva para un modelo de dos sabores de quarks que incluya corrientes quark-antiquark no locales vectoriales y axiales [70,71]. Se tiene

$$
\begin{aligned}
S_{E}= & \int d^{4} x\left\{\bar{\psi}(x)(-i \not \partial+\hat{m}) \psi(x)-\frac{G_{S}}{2}\left[j_{S}(x) j_{S}(x)+\vec{j}_{P}(x) \cdot \vec{j}_{P}(x)+j_{M}(x) j_{M}(x)\right]\right. \\
& \left.-\frac{G_{V}}{2}\left[\vec{j}_{V}^{\mu}(x) \cdot \vec{j}_{V \mu}(x)+\vec{j}_{A}^{\mu}(x) \cdot \vec{j}_{A \mu}(x)\right]-\frac{G_{0}}{2} j_{V}^{0 \mu}(x) j_{V \mu}^{0}(x)-\frac{G_{5}}{2} j_{A}^{0 \mu}(x) j_{A \mu}^{0}(x)\right\} .
\end{aligned}
$$

Esta acción es análoga a la del capítulo anterior manteniendo las mismas expresiones para las corrientes escalares $j_{S}(x)$ y $j_{M}(x)(I=0)$, y pseudoescalar $j_{P}^{a}(x)(I=1)$ presentes en la ecuación (3.26), e introduciendo además las corrientes vectoriales $j_{V \mu}^{a}(x)$ y $j_{V \mu}^{0}(x)$, y axiales $j_{A \mu}^{a}(x)$ y $j_{A \mu}^{0}(x)$, que transforman como singuletes o tripletes de isospín, y se definen como 


$$
\begin{aligned}
j_{V \mu}^{a}(x) & =\int d^{4} z h(z) \bar{\psi}\left(x+\frac{z}{2}\right) \tau^{a} \gamma_{\mu} \psi\left(x-\frac{z}{2}\right), \\
j_{A \mu}^{a}(x) & =\int d^{4} z h(z) \bar{\psi}\left(x+\frac{z}{2}\right) \tau^{a} \gamma_{\mu} \gamma_{5} \psi\left(x-\frac{z}{2}\right), \\
j_{V \mu}^{0}(x) & =\int d^{4} z h_{0}(z) \bar{\psi}\left(x+\frac{z}{2}\right) \gamma_{\mu} \psi\left(x-\frac{z}{2}\right), \\
j_{A \mu}^{0}(x) & =\int d^{4} z h_{5}(z) \bar{\psi}\left(x+\frac{z}{2}\right) \gamma_{\mu} \gamma_{5} \psi\left(x-\frac{z}{2}\right) .
\end{aligned}
$$

Las funciones $h(z), h_{0}(z)$ y $h_{5}(z)$ son los factores de forma covariantes responsables del carácter no local de las nuevas interacciones. Vale la pena destacar que, como se encontró en el capítulo anterior para $g(z)$, el factor de forma $h(z)$ debe ser el mismo para $j_{V \mu}^{a} \mathrm{y}$ $j_{A \mu}^{a}$ para garantizar la invarianza quiral. Los factores de forma $h_{0}(z)$ y $h_{5}(z)$ son en principio funciones independientes debido a que pertenecen a términos autoinvariantes ante transformaciones quirales.

Finalmente, se introdujeron nuevas constantes de acoplamiento: $G_{V}$ para el acoplamiento entre las corrientes isovectoriales vectoriales y axiales, y $G_{0}$ y $G_{5}$ para las interacciones de las corrientes isoescalares.

Siguiendo el procedimiento descrito en el capítulo anterior, procedemos a realizar una bosonización de la teoría fermiónica, introduciendo ahora también los campos bosónicos auxiliares $v_{\mu}^{0}(x), v_{\mu}^{a}(x)$ y $a_{\mu}^{0}(x), a_{\mu}^{a}(x)$. Luego de integrar los campos fermiónicos la función de partición puede escribirse como

$$
\mathcal{Z}=\int \mathcal{D} \sigma_{1} \mathcal{D} \sigma_{2} \mathcal{D} \vec{\pi} \mathcal{D} v_{\mu}^{0} \mathcal{D} a_{\nu}^{0} \mathcal{D} \vec{v}_{\alpha} \mathcal{D} \vec{a}_{\beta} \exp \left[-S_{E}^{\text {bos }}\right],
$$

donde $S_{E}^{\text {bos }}$ es la acción euclídea bosonizada. En el espacio de momentos, ésta está dada por

$$
\begin{aligned}
S_{E}^{\text {bos }}= & -\log \operatorname{det} A\left(p, p^{\prime}\right) \\
& +\int \frac{d^{4} p}{(2 \pi)^{4}}\left\{\frac{1}{2 G_{S}}\left[\sigma_{1}(p) \sigma_{1}(-p)+\vec{\pi}(p) \cdot \vec{\pi}(-p)+\sigma_{2}(p) \sigma_{2}(-p)\right]\right. \\
& +\frac{1}{2 G_{V}}\left[\vec{v}_{\mu}(p) \cdot \vec{v}^{\mu}(-p)+\vec{a}_{\mu}(p) \cdot \vec{a}^{\mu}(-p)\right] \\
& \left.+\frac{1}{2 G_{0}} v_{\mu}^{0}(p) v^{0 \mu}(-p)+\frac{1}{2 G_{5}} a_{\mu}^{0}(p) a^{0 \mu}(-p)\right\},
\end{aligned}
$$

donde el operador $A\left(p, p^{\prime}\right)$ es

$$
\begin{aligned}
A\left(p, p^{\prime}\right)= & (2 \pi)^{4} \delta^{(4)}\left(p-p^{\prime}\right)\left(-p+m_{c}\right)+g(\bar{p})\left[\sigma_{1}\left(p^{\prime}-p\right)+i \gamma_{5} \vec{\tau} \cdot \vec{\pi}\left(p^{\prime}-p\right)\right] \\
& +f(\bar{p}) \frac{p}{\varkappa} \sigma_{2}\left(p^{\prime}-p\right)+h(\bar{p}) \gamma^{\mu}\left[\vec{\tau} \cdot \vec{v}_{\mu}\left(p^{\prime}-p\right)+\gamma_{5} \vec{\tau} \cdot \vec{a}_{\mu}\left(p^{\prime}-p\right)\right] \\
& +h_{0}(\bar{p}) \gamma^{\mu} v_{\mu}^{0}\left(p^{\prime}-p\right)+h_{5}(\bar{p}) \gamma^{\mu} \gamma_{5} a_{\mu}^{0}\left(p^{\prime}-p\right),
\end{aligned}
$$


con $\bar{p} \equiv\left(p+p^{\prime}\right) / 2$.

Consideraremos nuevamente en primer lugar la aproximación de campo medio. Del mismo modo que en el modelo discutido en el capitulo anterior, únicamente los campos $\sigma_{1}(x)$ y $\sigma_{2}(x)$ pueden tener valores de expectación de vacío no triviales. Por lo tanto podemos escribir

$$
\begin{aligned}
v^{0 \mu}(x) & =\delta v^{0 \mu}(x), \\
\vec{v}^{\mu}(x) & =\delta \vec{v}^{\mu}(x), \\
a^{0 \mu}(x) & =\delta a^{0 \mu}(x), \\
\vec{a}^{\mu}(x) & =\delta \vec{a}^{\mu}(x) .
\end{aligned}
$$

A causa de esto, el cálculo de la acción a campo medio por unidad de volumen realizado con anterioridad no se ve modificado, y nos encontramos con la misma forma funcional que la expresada en la ecuación (3.36). Asimismo, las ecuaciones de gap y de los condensados quirales también vendrán dadas por (3.38) y (3.39). La diferencias con el caso ya estudiado se comenzarán a encontrar a partir del desarrollo cuadrático de la acción.

\subsubsection{Fluctuaciones cuadráticas}

Para calcular las masas de los mesones continuamos con el desarrollo de la acción euclídea a segundo orden en las fluctuaciones de los campos bosónicos. Obtenemos entonces una nueva expresión para $S_{E}^{\text {quad }}$ extendida al sector vectorial-axial

$$
\begin{aligned}
S_{E}^{\text {quad }} & =\frac{1}{2} \int \frac{d^{4} p}{(2 \pi)^{4}}\left\{G_{\sigma}\left(p^{2}\right) \delta \sigma(p) \delta \sigma(-p)+G_{\sigma^{\prime}}\left(p^{2}\right) \delta \sigma^{\prime}(p) \delta \sigma^{\prime}(-p)\right. \\
& +G_{\pi}\left(p^{2}\right) \delta \vec{\pi}(p) \cdot \delta \vec{\pi}(-p)+i G_{\pi a}\left(p^{2}\right)\left[p^{\mu} \delta \vec{a}_{\mu}(-p) \cdot \delta \vec{\pi}(p)-p^{\mu} \delta \vec{a}_{\mu}(p) \cdot \delta \vec{\pi}(-p)\right] \\
& +G_{0}^{\mu v}\left(p^{2}\right) \delta v_{\mu}^{0}(p) \delta v_{v}^{0}(-p)+G_{5}^{\mu v}\left(p^{2}\right) \delta a_{\mu}^{0}(p) \delta a_{v}^{0}(-p) \\
& \left.+G_{v}^{\mu v}\left(p^{2}\right) \delta \vec{v}_{\mu}(p) \cdot \delta \vec{v}_{v}(-p)+G_{a}^{\mu v}\left(p^{2}\right) \delta \vec{a}_{\mu}(p) \cdot \delta \vec{a}_{v}(-p)\right\}
\end{aligned}
$$

En virtud del carácter vectorial de las fluctuaciones de los campos, encontramos que ellos se encuentran acoplados a tensores de segundo orden que denominamos $G_{v}^{\mu v}, G_{a}^{\mu v}$, $G_{0}^{\mu \nu}$ y $G_{5}^{\mu \nu}$. Estos tensores se pueden descomponer en partes trasversa y longitudinal

$$
\begin{aligned}
& G_{v}^{\mu v}\left(p^{2}\right)=G_{\rho}\left(p^{2}\right)\left(g^{\mu v}-\frac{p^{\mu} p^{v}}{p^{2}}\right)+L_{+}\left(p^{2}\right) \frac{p^{\mu} p^{v}}{p^{2}} \\
& G_{a}^{\mu v}\left(p^{2}\right)=G_{\mathrm{a}_{1}}\left(p^{2}\right)\left(g^{\mu v}-\frac{p^{\mu} p^{v}}{p^{2}}\right)+L_{-}\left(p^{2}\right) \frac{p^{\mu} p^{v}}{p^{2}}
\end{aligned}
$$


donde definimos las funciones

$$
\begin{aligned}
& G_{\left(\begin{array}{c}
\mathrm{a}_{1} \\
\mathrm{a}_{1}
\end{array}\right.}\left(p^{2}\right)=\frac{1}{G_{V}}-8 N_{c} \int \frac{d^{4} q}{(2 \pi)^{4}} h^{2}(q) \frac{z\left(q^{+}\right) z\left(q^{-}\right)}{D\left(q^{+}\right) D\left(q^{-}\right)}\left[\frac{q^{2}}{3}+\frac{2(p \cdot q)^{2}}{3 p^{2}}-\frac{p^{2}}{4} \pm m\left(q^{-}\right) m\left(q^{+}\right)\right], \\
& L_{ \pm}\left(p^{2}\right)=\frac{1}{G_{V}}-8 N_{c} \int \frac{d^{4} q}{(2 \pi)^{4}} h^{2}(q) \frac{z\left(q^{+}\right) z\left(q^{-}\right)}{D\left(q^{+}\right) D\left(q^{-}\right)}\left[q^{2}-\frac{2(p \cdot q)^{2}}{p^{2}}+\frac{p^{2}}{4} \pm m\left(q^{-}\right) m\left(q^{+}\right)\right]
\end{aligned}
$$

con $q^{ \pm}=q \pm p / 2$. Las funciones $G_{\rho, \mathrm{a}_{1}}\left(p^{2}\right)$ y $L_{ \pm}\left(p^{2}\right)$ corresponden a las proyecciones trasversas y longitudinales de los campos vectoriales y vectorial-axiales, describiendo estados mesónicos de espín 1 y 0 , respectivamente. Las masas correspondientes a los estados físicos que describen a los mesones vectoriales $\rho^{0}$ y $\rho^{ \pm}\left(J^{C P}=1^{--}\right.$) (que corresponden a un triplete de isospín en la aproximación $m_{d}=m_{u}$ que se está considerando) pueden obtenerse resolviendo la ecuación

$$
G_{\rho}\left(-m_{\rho}^{2}\right)=0
$$

También aquí debemos llevar a cabo la renormalización de los campos fijando el residuo de $G_{\rho}\left(p^{2}\right)$ en el polo del propagador, esto es,

$$
\delta v_{\mu}^{a}(p)=Z_{\rho}^{1 / 2} \tilde{v}_{\mu}^{a}(p)
$$

donde

$$
Z_{\rho}^{-1}=g_{\rho q q}^{-2}=\left.\frac{d G_{\rho}\left(p^{2}\right)}{d p^{2}}\right|_{p^{2}=-m_{\rho}^{2}} .
$$

Para los canales de isospín cero, es sencillo de comprobar que las expresiones de $G_{0}^{\mu v}\left(p^{2}\right)$ se pueden calcular a partir de $G_{v}^{\mu v}\left(p^{2}\right)$, únicamente reemplazando $G_{V}$ por $G_{0}, \mathrm{y} h(q)$ por $h_{0}(q)$. De este modo puede definirse para el mesón vectorial $\omega$ una función $G_{\omega}\left(p^{2}\right)$, y así calcular su masa y renormalización de función de onda de acuerdo a las ecuaciones (4.12) y (4.14). Relaciones similares son aplicables para el sector axial, donde $G_{5}^{\mu v}\left(p^{2}\right)$ puede obtenerse a partir de $G_{a}^{\mu v}\left(p^{2}\right)$ reemplazando $G_{V}$ por $G_{5}$ y $h(q)$ por $h_{5}(q)$. El estado físico más liviano observado asociado a este sector (con números cuánticos $I=0, J^{P}=1^{+}$) es el mesón axial $f_{1}$, por lo que denominamos $G_{f_{1}}\left(p^{2}\right)$ al factor de forma correspondiente a la proyección transversa de $G_{5}^{\mu v}\left(p^{2}\right)$.

En el caso del sector pseudoescalar, mientras que el término cuadrático en $\delta \pi$ no se ve modificado con respecto al calculado en la expresión (3.41), surge, debido a la inclusión del sector vectorial-axial, una mezcla entre el campo pseudoescalar y la proyección longitudinal del campo axial $[72,73]$. El término de mezcla $G_{\pi a}\left(p^{2}\right)$, que se origina a partir de los términos cruzados del determinante fermiónico entre los campos $\vec{\pi}(p)$ y $\vec{a}_{\mu}$ [ver 
ecuación (4.8)], está dado por una integral a un loop de la forma

$$
G_{\pi a}\left(p^{2}\right)=\frac{8 N_{c}}{p^{2}} \int \frac{d^{4} q}{(2 \pi)^{4}} g(q) h(q) \frac{z\left(q^{+}\right) z\left(q^{-}\right)}{D\left(q^{+}\right) D\left(q^{-}\right)}\left[\left(q^{+} \cdot p\right) m\left(q^{-}\right)-\left(q^{-} \cdot p\right) m\left(q^{+}\right)\right],
$$

donde una vez más utilizamos $q^{ \pm}=q \pm p / 2$. Pueden obtenerse estados físicos desacoplados $\tilde{\vec{a}}_{\mu} \mathrm{y} \tilde{\vec{\pi}}$ a través de las relaciones $[72,73]$

$$
\begin{aligned}
\delta \pi^{b}(p) & =Z_{\pi}^{1 / 2} \tilde{\pi}^{b}(p) \\
\delta a_{\mu}^{b}(p) & =Z_{a}^{1 / 2} \tilde{a}_{\mu}^{b}(p)-i \lambda\left(p^{2}\right) p_{\mu} Z_{\pi}^{1 / 2} \tilde{\pi}^{b}(p)
\end{aligned}
$$

donde se ha introducido una función de mezcla $\lambda\left(p^{2}\right)$, que se determina reemplazando las relaciones (4.16) en la ecuación (4.8) y exigiendo que se cancele el término cruzado en el desarrollo cuadrático de la acción. Encontramos de este modo

$$
\lambda\left(p^{2}\right)=\frac{G_{\pi a}\left(p^{2}\right)}{L_{-}\left(p^{2}\right)}
$$

y también la expresión para $G_{\tilde{\pi}}\left(p^{2}\right)$ dada por

$$
G_{\tilde{\pi}}\left(p^{2}\right)=G_{\pi}\left(p^{2}\right)-\frac{G_{\pi a}\left(p^{2}\right)^{2}}{L_{-}\left(p^{2}\right)} p^{2} .
$$

Evaluando en el polo $G_{\tilde{\pi}}\left(-m_{\pi}^{2}\right)=0$ calculamos la masa del pion, mientras que su función de renormalización de onda se obtiene como

$$
Z_{\pi}^{-1}=g_{\pi q q}^{-2}=\left.\frac{d G_{\tilde{\pi}}\left(p^{2}\right)}{d p^{2}}\right|_{p^{2}=-m_{\pi}^{2}} .
$$

En el caso de los mesones axiales $\mathrm{a}_{1}$ (triplete $I=1$ ), al no encontrarse ninguna mezcla que involucre la proyección transversa de los campos $a_{\mu}^{b}$, la masa correspondiente y la renormalización de función de onda se consiguen gracias a relaciones análogas a las utilizadas en el sector vectorial, es decir las ecuaciones (4.12) y (4.14), con $G_{\mathrm{a}_{1}}\left(p^{2}\right)$ dada por la ecuación (4.10).

\subsection{Decaimientos}

\subsubsection{Decaimiento débil del pion}

La constante de decaimiento débil del pion se define a partir del campo correspondiente al estado físico de la partícula. Es por ello que, de acuerdo con la ecuación (4.16), ahora debemos incluir las contribuciones a esta constante provenientes de la mezcla con el campo vectorial-axial. 
Tal como vimos en el capítulo anterior $f_{\pi}$ se calcula a partir de los elementos de matriz que se obtienen derivando la acción con respecto a los campos de gauge y el campo del pion físico. En este modelo esto es equivalente a

$$
\left\langle 0\left|\mathcal{J}_{A \mu}^{a}(x)\right| \tilde{\pi}^{b}(p)\right\rangle=\left.Z_{\pi}^{1 / 2} \frac{\delta^{2} S_{E}^{\text {bos }}}{\delta \mathcal{W}_{\mu}^{a}(x) \delta \pi^{b}(p)}\right|_{\mathcal{W}_{\mu}^{a}=\pi^{b}=0}+\left.i \lambda p_{\nu} Z_{\pi}^{1 / 2} \frac{\delta^{2} S_{E}^{\text {bos }}}{\delta \mathcal{W}_{\mu}^{a}(x) \delta a_{\nu}^{b}(p)}\right|_{\mathcal{W}_{\mu}^{a}=a_{v}^{b}=0} .
$$

De este modo, no es suficiente con calcular únicamente el desarrollo de la acción bosonizada en los campos de gauge $\mathcal{W}_{\mu}^{a}(x)$ y el campo pseudoescalar $\vec{\pi}$, sino que también debemos considerar el desarrollo lineal en el campo $\vec{a}_{\mu}$.

El proceso por el cual se construye la transformación de gauge de la acción efectiva $S_{E}$ es semejante al detallado en el capítulo anterior. Una vez realizada dicha transformación y haciendo las derivadas pertinentes se obtienen dos nuevas contribuciones a la constante de decaimiento, esquematizadas en la Figura (4.1).
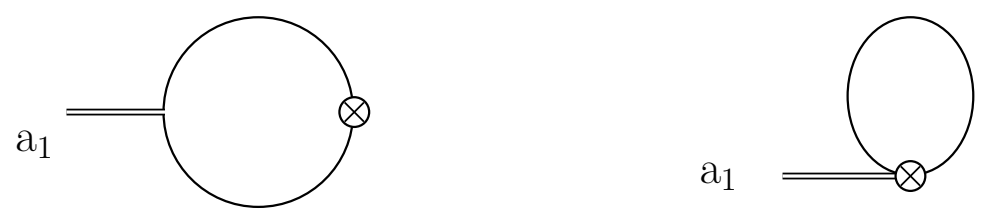

Figura 4.1: Representación diagramática de las contribuciones a la constante de decaimiento débil del pion provenientes del campo vectorial-axial.

Finalmente, luego de un cálculo relativamente extenso, obtenemos

$$
f_{\pi}=\frac{m_{c} g_{\pi q \bar{q}}}{m_{\pi}^{2}}\left[F_{0}\left(-m_{\pi}^{2}\right)+\lambda\left(p^{2}\right) F_{1}\left(-m_{\pi}^{2}\right)\right]
$$

donde

$$
\begin{aligned}
& F_{0}\left(p^{2}\right)=8 N_{c} \int \frac{d^{4} q}{(2 \pi)^{4}} g(q) \frac{z\left(q^{+}\right) z\left(q^{-}\right)}{D\left(q^{+}\right) D\left(q^{-}\right)}\left[\left(q^{+} \cdot q^{-}\right)+m\left(q^{+}\right) m\left(q^{-}\right)\right] \\
& F_{1}\left(p^{2}\right)=8 N_{c} \int \frac{d^{4} q}{(2 \pi)^{4}} h(q) \frac{z\left(q^{+}\right) z\left(q^{-}\right)}{D\left(q^{+}\right) D\left(q^{-}\right)}\left[\left(q^{+} \cdot p\right) m\left(q^{-}\right)-\left(q^{-} \cdot p\right) m\left(q^{+}\right)\right] .
\end{aligned}
$$

En ausencia de campos vectoriales el término de mezcla en la ecuación (4.21) desaparece y nuestra expresión se reduce a la obtenida en el capítulo anterior.

\subsubsection{Vértice $\rho^{0}$-fotón y constante de decaimiento electromagnética del $\rho$}

Otra importante cantidad para ser estudiada es el vértice $\rho^{0}$-fotón. En nuestro modelo no local, los acoplamientos mesón-fotón reciben las contribuciones del transporte paralelo en las ecuaciones (3.48), por lo tanto consideramos relevante confirmar que la conservación 
de la corriente vectorial se satisface. Además, a partir de este vértice es posible obtener una predicción para la amplitud del decaimiento electromagnético $\rho^{0} \rightarrow e^{+} e^{-}$.

El vértice $\rho^{0}$-fotón está dado por el elemento de matriz de la corriente electromagnética entre el estado del mesón vectorial y el vacío,

$$
\left\langle 0\left|\mathcal{J}_{\operatorname{em} \mu}(x)\right| \tilde{v}_{v}^{3}(p)\right\rangle=i e^{-i p \cdot x} \Pi_{\mu \nu}^{3}(p) .
$$

Para calcular este elemento de matriz se puede seguir el procedimiento discutido anteriormente, tomando ahora

$$
\mathcal{G}_{\mu}=\hat{Q} \mathcal{A}_{\mu},
$$

donde $\hat{Q}=\operatorname{diag}(e 2 / 3,-e 1 / 3)$ es la matriz de carga en la representación fundamental del grupo $\mathrm{SU}(2)_{f}$, y e es la carga del protón.

Una vez más es posible distinguir dos contribuciones a $\Pi_{\mu v}^{3}$, digamos $\Pi_{\mu \nu}^{(\mathrm{I}) 3} \mathrm{y} \Pi_{\mu \nu}^{(\mathrm{II}) 3}$, que surgen del diagrama de dos vértices y del de tipo tadpole respectivamente (ver Figura 4.2). Obtenemos

$$
\begin{aligned}
\Pi_{\mu \nu}^{(\mathrm{I}) 3}(p)= & 4 N_{C} e Z_{\rho}^{1 / 2} \int \frac{d^{4} q}{(2 \pi)^{4}} \frac{z\left(q^{+}\right) z\left(q^{-}\right)}{D\left(q^{+}\right) D\left(q^{-}\right)} h(q) \\
& \times\left\{\frac{1}{2}\left[\frac{1}{z\left(q^{+}\right)}+\frac{1}{z\left(q^{-}\right)}\right]\left[q_{\mu}^{+} q_{v}^{-}+q_{v}^{+} q_{\mu}^{-}-\left(q^{+} \cdot q^{-}\right) \delta_{\mu v}-m\left(q^{+}\right) m\left(q^{-}\right) \delta_{\mu v}\right]\right. \\
& +\bar{\sigma}_{1}\left[m\left(q^{+}\right) q_{v}^{-}+m\left(q^{-}\right) q_{v}^{+}\right] \alpha_{g \mu}(q, p) \\
& \left.+\bar{\sigma}_{2}\left[-\frac{\left(q^{-}\right)^{2}}{2} q_{v}^{+}-\frac{\left(q^{+}\right)^{2}}{2} q_{v}^{-}+m\left(q^{+}\right) m\left(q^{-}\right) q_{v}\right] \alpha_{f \mu}(q, p)\right\} \\
\Pi_{\mu v}^{(\mathrm{II}) 3}(p)= & -4 N_{C} e Z_{\rho}^{1 / 2} \int \frac{d^{4} q}{(2 \pi)^{4}} \frac{z(q)}{D(q)} q_{v} \alpha_{h \mu}(q, p)
\end{aligned}
$$

Aquí hemos definido, para una dada función $r(p)$,

$$
\alpha_{r \mu}(q, p)=\int \frac{d^{4} \ell}{(2 \pi)^{4}}\left[r(q+\ell / 2) S_{\mu}(p-\ell, \ell)+r(q-\ell / 2) S_{\mu}(\ell, p-\ell)\right],
$$

con

$$
S_{\mu}\left(k, k^{\prime}\right)=-i \int d^{4} z e^{i k^{\prime} z} \int_{0}^{z} d t_{\mu} e^{-i\left(k+k^{\prime}\right) t},
$$

donde $t$ recorre un camino que une el origen de coordenadas con el punto ubicado en $z$.

Los tensores $\Pi_{\mu v}^{(\mathrm{I}) 3}$ y $\Pi_{\mu \nu}^{(\mathrm{II}) 3}$ son en general no transversos. Sin embargo, la suma de ambas contribuciones satisface $p^{\mu} \Pi_{\mu v}^{3}=0$, como se requiere de la conservación de la corriente electromagnética. Esto puede verificarse notando que

$$
\left(k+k^{\prime}\right)^{\mu} S_{\mu}\left(k, k^{\prime}\right)=-i \int d^{4} z e^{i k^{\prime} z} \int_{0}^{z\left(k+k^{\prime}\right)} d \omega e^{-i \omega}=(2 \pi)^{4}\left[\delta^{(4)}(k)-\delta^{(4)}\left(k^{\prime}\right)\right],
$$



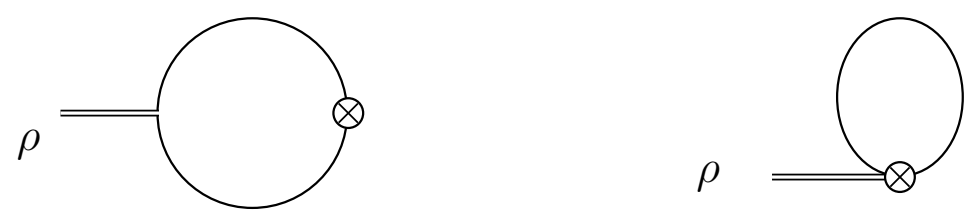

Figura 4.2: Diagramas que contribuyen al vértice $\rho^{0}$-fotón.

lo cual conduce a

$$
p^{\mu} \alpha_{r \mu}(q, p)=r\left(q^{+}\right)-r\left(q^{-}\right)
$$

Por lo tanto, el resultado en la ecuación (4.30) no depende en la integral de camino en (4.28) [un mecanismo similar lleva a una independencia de camino en las funciones en las ecuaciones (4.22)]. Utilizando la relación en la ecuación (4.30), luego de un adecuado cambio de variables se obtiene

$$
p^{\mu}\left(\Pi_{\mu v}^{(\mathrm{I}) 3}+\Pi_{\mu \nu}^{(\mathrm{II}) 3}\right)=0 .
$$

Una cancelación similar se encontró en la Ref. [64], en el marco de un modelo nlNJL que incluye mesones vectoriales sin WFR.

Nos concentraremos ahora en la constante de decaimiento electromagnético $f_{v}$, definida a partir del decaimiento $\rho^{0} \rightarrow e^{+} e^{-}$. Se tiene

$$
\Gamma\left(\rho^{0} \rightarrow e^{+} e^{-}\right)=\frac{4 \pi}{3} \alpha^{2} m_{\rho} f_{v}^{2},
$$

donde $\alpha=e^{2} /(4 \pi)$ es la constante de estructura fina electromagnética. Podemos relacionar el valor de la constante $f_{v}$ con la traza de $\Pi_{\mu v}^{3}(p)$ a través de

$$
3 m_{\rho}^{2} e f_{v}=\left.g_{\mu v} \Pi_{\mu v}^{3}(p)\right|_{p^{2}=-m_{\rho}^{2}} .
$$

Resulta ahora necesario evaluar la parte transversa del tensor $\Pi_{\mu v}^{3}$. Para ello se debe tomar un camino para la integral sobre $t_{\mu}$ en la ecuación (4.28), siendo la trayectoria más sencilla un camino lineal. Dicha elección conduce a

$$
\alpha_{r \mu}(q, p)=\int_{-1}^{1} d \lambda\left(q_{\mu}+\lambda \frac{p_{\mu}}{2}\right) r^{\prime}\left(q+\lambda \frac{p}{2}\right)
$$

donde $r^{\prime}(p)$ denota la derivada de $r$ con respecto a $p^{2}$. Luego de algo de álgebra, obtenemos

$$
f_{v}=\frac{Z_{\rho}^{1 / 2}}{3 m_{\rho}^{2}}\left[J^{(\mathrm{I})}\left(-m_{\rho}^{2}\right)+J^{(\mathrm{II})}\left(-m_{\rho}^{2}\right)\right] \text {, }
$$


con

$$
\begin{aligned}
J^{(\mathrm{I})}\left(p^{2}\right)= & -4 N_{c} \int \frac{d^{4} q}{(2 \pi)^{4}} h(q)\left\{\frac{3}{2} \frac{\left[z\left(q^{+}\right)+z\left(q^{-}\right)\right]}{D\left(q^{+}\right) D\left(q^{-}\right)}\left[\left(q^{+} \cdot q^{-}\right)+m\left(q^{+}\right) m\left(q^{-}\right)\right]\right. \\
& +\frac{1}{2} \frac{z\left(q^{+}\right)}{D\left(q^{+}\right)}+\frac{1}{2} \frac{z\left(q^{-}\right)}{D\left(q^{-}\right)}+\frac{q^{2}}{(q \cdot p)}\left[\frac{z\left(q^{+}\right)}{D\left(q^{+}\right)}-\frac{z\left(q^{-}\right)}{D\left(q^{-}\right)}\right] \\
& +\frac{z\left(q^{+}\right) z\left(q^{-}\right)}{D\left(q^{+}\right) D\left(q^{-}\right)}\left[(q \cdot p)-\frac{q^{2} p^{2}}{(q \cdot p)}\right]\left[-\bar{\sigma}_{1}\left[m\left(q^{+}\right)+m\left(q^{-}\right)\right] \alpha_{g}^{+}(q, p)\right. \\
& \left.\left.+\bar{\sigma}_{2}\left[q^{2}+\frac{p^{2}}{4}-m\left(q^{+}\right) m\left(q^{-}\right)\right] \alpha_{f}^{+}(q, p)\right]\right\}, \\
J^{(\mathrm{II})}\left(p^{2}\right)= & -4 N_{c} \int \frac{d^{4} q}{(2 \pi)^{4}} \frac{z(q)}{D(q)}\left\{\frac{q^{2}}{(q \cdot p)}\left[h\left(q^{+}\right)-h\left(q^{-}\right)\right]+\left[(q \cdot p)-\frac{q^{2} p^{2}}{(q \cdot p)}\right] \alpha_{h}^{+}(q, p)\right\} .
\end{aligned}
$$

Los superíndices (I) y (II) corresponden a las contribuciones de los diagramas en las Figuras $4.2 \mathrm{a}$ y $4.2 \mathrm{~b}$, respectivamente, mientras que las funciones $\alpha_{f}^{+}(q, p)$ vienen definidas como

$$
\alpha_{f}^{+}(q, p)=\int_{-1}^{1} d \lambda \frac{\lambda}{2} f^{\prime}\left(q-\lambda \frac{p}{2}\right)
$$

\subsubsection{Decaimiento $\pi^{0} \rightarrow \gamma \gamma$}

Vamos a analizar en el contexto de nuestro modelo el decaimiento anómalo $\pi^{0} \rightarrow \gamma \gamma$. En el modelo NJL este decaimiento suele ser problemático: para poder reproducir el resultado observado experimentalmente es necesario realizar integraciones a un loop en el espacio de momentos hasta el infinito, en lugar de integrar hasta un cut-off $\Lambda_{3}$ como en el caso del condensado $\langle\bar{q} q\rangle$ [74]. La amplitud de decaimiento puede calcularse a partir del elemento de matriz

$$
\left\langle 0\left|\mathcal{J}_{\operatorname{em} \mu}(x) \mathcal{J}_{\operatorname{em} v}(0)\right| \tilde{\pi}^{3}(p)\right\rangle=\left.\frac{\delta^{3} S_{E}^{\text {bos }}}{\delta \mathcal{A}_{\mu}(x) \delta \mathcal{A}_{\nu}(0) \delta \tilde{\pi}^{3}(p)}\right|_{\mathcal{A}_{\mu, \nu}=\tilde{\pi}^{3}=0} .
$$

En principio hay varios diagramas que contribuyen a la amplitud al nivel de un loop. Tal como ocurre cuando calculamos la constante de decaimiento débil del pion $f_{\pi}$, como el campo asociado al $\pi^{0}, \tilde{\pi}^{3}(p)$, es una combinación de los campos $\pi^{3}$ y $a_{\mu}^{3}$, debemos considerar la parte lineal del desarrollo de la acción bosonizada en $\delta \pi^{3}$ y $\delta a_{\mu}^{3}$. Los diagramas que tienen contribuciones distintas de cero se muestran en la Figura 4.3. Si los fotones salientes tienen impulsos $k_{1}$ y $k_{2}$ con vectores de polarización $\varepsilon_{\mu}^{\left(\lambda_{1}\right)}\left(k_{1}\right)$ y $\varepsilon_{v}^{\left(\lambda_{2}\right)}\left(k_{2}\right)$, respectivamente, la amplitud de decaimiento puede escribirse como

$$
\mathcal{M}\left(\pi^{0} \rightarrow \gamma \gamma\right)=i 4 \pi \alpha \tilde{F}\left(k_{1}, k_{2}\right) \epsilon^{\mu v \alpha \beta} \varepsilon_{\mu}^{\left(\lambda_{1}\right)}\left(k_{1}\right)^{*} \varepsilon_{v}^{\left(\lambda_{2}\right)}\left(k_{2}\right)^{*} k_{1 \alpha} k_{2 \beta}
$$


donde el factor de forma $\tilde{F}\left(k_{1}, k_{2}\right)$ está dado por la suma de las contribuciones de los campos $\pi^{3}$ y $a_{\mu}^{3}$ al estado $\tilde{\pi}^{3}$,

$$
\tilde{F}\left(k_{1}, k_{2}\right)=Z_{\pi}^{1 / 2}\left[F_{\pi}\left(k_{1}, k_{2}\right)+\lambda\left(p^{2}\right) F_{a}\left(k_{1}, k_{2}\right)\right]
$$

$\operatorname{con} p=k_{1}+k_{2}$.

El primer término entre corchetes, correspondiente al diagrama en la Figura 4.3a, ha sido calculado (a menos del factor de isospín) en la Ref. [69]. Se obtiene

$$
F_{\pi}\left(k_{1}, k_{2}\right)=\frac{2 N_{c}}{3} \int \frac{d^{4} q}{(2 \pi)^{4}} h\left(q+\frac{k_{2}}{2}-\frac{k_{1}}{2}\right) \frac{z(q) z\left(q-k_{1}\right) z\left(q+k_{2}\right)}{D(q) D\left(q-k_{1}\right) D\left(q+k_{2}\right)} A\left(q, k_{1}, k_{2}\right),
$$

donde

$$
\begin{aligned}
A\left(q, k_{1}, k_{2}\right)= & \left(\frac{1}{z(q)}+\frac{1}{z\left(q-k_{1}\right)}\right)\left(\frac{1}{z(q)}+\frac{1}{z\left(q+k_{2}\right)}\right)\left\{m(q)-\frac{q^{2}}{2} \times\right. \\
& {\left.\left[\frac{m\left(q+k_{2}\right)-m(q)}{\left(q \cdot k_{2}\right)}-\frac{m\left(q-k_{1}\right)-m(q)}{\left(q \cdot k_{1}\right)}\right]\right\} . }
\end{aligned}
$$

Por otro lado, el factor de forma $F_{a}\left(k_{1}, k_{2}\right)$ surge de la suma de las contribuciones corres-

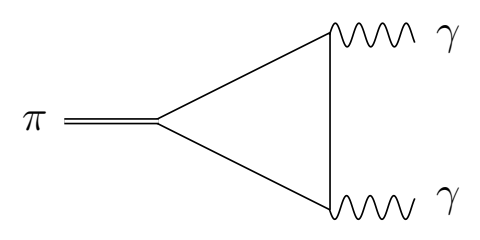

(a)

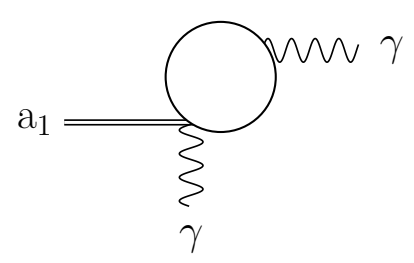

(b)

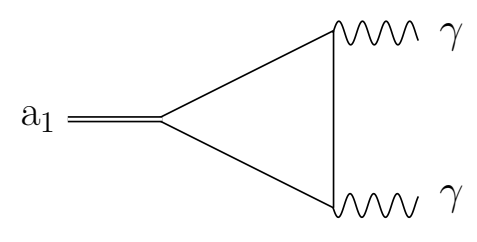

(c)

Figura 4.3: Diagramas que contribuyen al decaimiento $\pi^{0} \rightarrow \gamma \gamma$.

pondientes a los diagramas en las Figuras 4.3b y 4.3c. Aunque éstas son divergentes por separado, analíticamente se observa que las partes divergentes se cancelan en la suma, que resulta finita. Obtenemos

$$
\begin{aligned}
F_{a}\left(k_{1}, k_{2}\right)= & -\frac{2 N_{c}}{3} \int \frac{d^{4} q}{(2 \pi)^{4}}\left\{h\left(q+k_{2} / 2-k_{1} / 2\right) \frac{z(q) z\left(q-k_{1}\right) z\left(q+k_{2}\right)}{D(q) D\left(q-k_{1}\right) D\left(q+k_{2}\right)} \times\right. \\
& {\left[\left(m\left(q-k_{1}\right)+m\left(q+k_{2}\right)\right) A\left(q, k_{1}, k_{2}\right)+\right.} \\
& \left.\frac{q^{2}}{2}\left(\frac{B\left(q, q-k_{1}, q+k_{2}\right)}{\left(q \cdot k_{2}\right)}-\frac{B\left(q, q+k_{2}, q-k_{1}\right)}{\left(q \cdot k_{1}\right)}\right)\right]+ \\
& \left.q^{2}\left[\frac{h\left(q+k_{2} / 2\right)}{\left(q \cdot k_{2}\right)} C\left(q, k_{1}\right)+\frac{h\left(q+k_{1} / 2\right)}{\left(q \cdot k_{1}\right)} C\left(q, k_{2}\right)\right]\right\}
\end{aligned}
$$




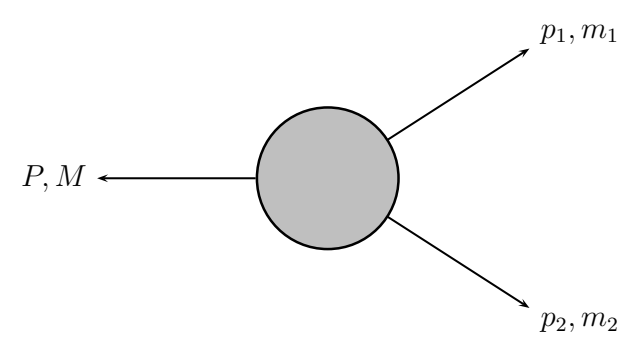

Figura 4.4: Esquema de decaimiento en dos cuerpos.

donde

$$
\begin{aligned}
B(q, r, s) & =\left(\frac{1}{z(q)}+\frac{1}{z(r)}\right)\left(\frac{1}{z(q)}-\frac{1}{z(s)}\right) D(s) \\
C(q, k) & =\left(\frac{1}{z(q+k / 2)}+\frac{1}{z(q-k / 2)}\right) \frac{z(q+k / 2) z(q-k / 2)}{D(q+k / 2) D(q-k / 2)} .
\end{aligned}
$$

Finalmente, luego de integrar en el espacio de momentos y sumar sobre las polarizaciones de los fotones salientes, la amplitud de decaimiento $\pi^{0} \rightarrow \gamma \gamma$ resulta

$$
\Gamma\left(\pi^{0} \rightarrow \gamma \gamma\right)=\frac{\pi}{4} \alpha^{2} m_{\pi}^{3} \tilde{F}\left(k_{1}, k_{2}\right)^{2} .
$$

Como los fotones están on-shell, es decir $k_{1}^{2}=k_{2}^{2}=0$, de la invarianza de Lorentz se puede ver que $\tilde{F}\left(k_{1}, k_{2}\right)$ sólo puede ser función del producto escalar $\left(k_{1} \cdot k_{2}\right)=-m_{\pi}^{2} / 2$.

\subsubsection{Decaimiento $\rho \rightarrow \pi \pi$}

En general, varias amplitudes de transición pueden calcularse desarrollando la acción bosonizada a órdenes mayores en las fluctuaciones de los campos mesónicos. En esta sección nos concentraremos en los procesos $\rho^{0} \rightarrow \pi^{+} \pi^{-}$y $\rho^{ \pm} \rightarrow \pi^{ \pm} \pi^{0}$, responsables de más del $99 \%$ de los decaimientos de los mesones $\rho$. Para ello desarrollaremos la acción hasta el orden cúbico.

En la Figura 4.4 se esquematiza un decaimiento de una partícula de masa $M$ y momento $P$ en dos cuerpos [75]. El ancho diferencial de decaimiento se escribe en términos de una amplitud invariante $\mathcal{M}$ según:

$$
d \Gamma=\frac{1}{32 \pi^{2}}|\mathcal{M}|^{2} \frac{\left|p_{1}\right|}{M^{2}} d \Omega,
$$

donde $d \Omega$ es el diferencial de ángulo sólido de uno de los cuerpos salientes.

Las amplitudes correspondientes al decaimiento de interés $\mathcal{M}\left[v_{\mu}^{a}(p) \rightarrow \pi^{b}\left(q_{1}\right) \pi^{c}\left(q_{2}\right)\right]$ se obtienen calculando las correspondientes derivadas funcionales de la acción efectiva, 
que pueden escribirse en términos de dos factores de forma $\tilde{F}_{\rho \pi \pi}\left(p^{2}, q_{1}^{2}, q_{2}^{2}\right)$ y $\tilde{G}_{\rho \pi \pi}\left(p^{2}, q_{1}^{2}, q_{2}^{2}\right)$ :

$$
\begin{aligned}
\left.\frac{\delta^{3} S_{E}^{\text {bos }}}{\delta \tilde{v}_{\mu}^{a}(p) \delta \tilde{\pi}^{b}\left(q_{1}\right) \delta \tilde{\pi}^{c}\left(q_{2}\right)}\right|_{\delta v_{\mu}=\delta \pi=0} & =(2 \pi)^{4} \delta^{(4)}\left(p+q_{1}+q_{2}\right) \epsilon_{a b c} \times \\
& {\left[\tilde{F}_{\rho \pi \pi}\left(p^{2}, q_{1}^{2}, q_{2}^{2}\right) \frac{\left(q_{1 \mu}+q_{2 \mu}\right)}{2}+\tilde{G}_{\rho \pi \pi}\left(p^{2}, q_{1}^{2}, q_{2}^{2}\right) \frac{\left(q_{1 \mu}-q_{2 \mu}\right)}{2}\right] . }
\end{aligned}
$$

Sólo la parte transversa, dada por el factor de forma $\tilde{G}_{\rho \pi \pi}\left(p^{2}, q_{1}^{2}, q_{2}^{2}\right)$, contribuye al ancho de decaimiento $\rho \rightarrow \pi \pi$. En el límite isospín tenemos

$$
\Gamma_{\rho^{0} \rightarrow \pi^{+} \pi^{-}}=\Gamma_{\rho^{ \pm} \rightarrow \pi^{ \pm} \pi^{0}}=\frac{1}{48 \pi} m_{\rho} g_{\rho \pi \pi}^{2}\left(1-\frac{4 m_{\pi}^{2}}{m_{\rho}^{2}}\right)^{3 / 2}
$$

donde $g_{\rho \pi \pi} \equiv \tilde{G}_{\rho \pi \pi}\left(-m_{\rho}^{2},-m_{\pi}^{2},-m_{\pi}^{2}\right)$.

El factor de forma $\tilde{G}_{\rho \pi \pi}\left(p^{2}, q_{1}^{2}, q_{2}^{2}\right)$ surge del vértice efectivo $\tilde{\rho} \tilde{\pi} \tilde{\pi}$, donde $\tilde{\rho}$ y $\tilde{\pi}$ son campos renormalizados. Como en los procesos analizados anteriormente, dado que la acción efectiva se encuentra expresada en potencias de los campos no renormalizados, es conveniente escribir el vértice efectivo en términos de los campos originales $\rho, \pi$ y $a_{\mu}$. De este modo, el factor de forma recibe contribuciones de los diagramas esquematizados en la Figura 4.5. Se tiene

$$
\begin{aligned}
\tilde{G}_{\rho \pi \pi}\left(p^{2}, q_{1}^{2}, q_{2}^{2}\right)= & Z_{\rho}^{1 / 2} Z_{\pi}\left[G_{\rho \pi \pi}\left(p^{2}, q_{1}^{2}, q_{2}^{2}\right)\right. \\
& \left.+\lambda\left(p^{2}\right) G_{\rho \pi a}\left(p^{2}, q_{1}^{2}, q_{2}^{2}\right)+\lambda\left(p^{2}\right)^{2} G_{\rho a a}\left(p^{2}, q_{1}^{2}, q_{2}^{2}\right)\right],
\end{aligned}
$$

donde $G_{\rho \pi \pi}\left(p^{2}, q_{1}^{2}, q_{2}^{2}\right), G_{\rho \pi a}\left(p^{2}, q_{1}^{2}, q_{2}^{2}\right)$ y $G_{\rho a a}\left(p^{2}, q_{1}^{2}, q_{2}^{2}\right)$ son funciones a un loop que surgen del desarrollo de la acción efectiva.
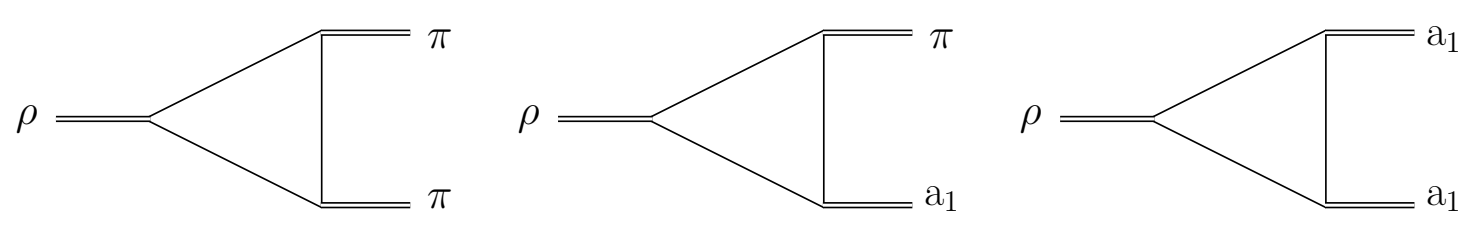

Figura 4.5: Diagramas que contribuyen al decaimiento $\rho \rightarrow \pi \pi$.

La forma analítica de estas funciones pueden obtenerse luego de un largo cálculo. Para la amplitud de decaimiento $\rho \rightarrow \pi \pi$, debemos evaluar las funciones en $p^{2}=-m_{\rho}^{2}$, y $q_{1}^{2}=q_{2}^{2}=\left(p-q_{1}\right)^{2}=-m_{\pi}^{2}$. Resulta conveniente introducir el momento $v=q_{1}-p / 2$, que satisface $p \cdot v=0, \mathrm{y} v^{2}=m_{\rho}^{2} / 4-m_{\pi}^{2}$. Entonces, las funciones $G_{\rho x y}\left(p^{2}, q_{1}^{2}, q_{2}^{2}\right)$, donde los subíndices $x$ e $y$ refieren a $\pi$ o $a$, pueden escribirse como

$$
\begin{aligned}
G_{\rho x y}\left(p^{2}, q_{1}^{2}, q_{2}^{2}\right)= & 16 N_{c} \int \frac{d^{4} q}{(2 \pi)^{4}} h(q) g\left(q+\frac{v}{2}+\frac{p}{4}\right) g\left(q+\frac{v}{2}-\frac{p}{4}\right) \times \\
& \frac{z\left(q^{+}\right) z\left(q^{-}\right) z(q+v)}{D\left(q^{+}\right) D\left(q^{-}\right) D(q+v)} f_{x y}(q, p, v),
\end{aligned}
$$


donde hemos definido $q^{ \pm}=q \pm p / 2$. Las expresiones resultantes para $f_{x y}(q, p, v)$ son

$$
\begin{aligned}
f_{\pi \pi}= & {\left[\left(q^{+} \cdot q^{-}\right)+m\left(q^{+}\right) m\left(q^{-}\right)\right]\left[1+\frac{(q \cdot v)}{v^{2}}\right] } \\
& -\frac{(q \cdot v)}{v^{2}}\left\{2[q \cdot(q+v)]+m(q+v)\left[m\left(q^{+}\right)+m\left(q^{-}\right)\right]\right\}, \\
f_{\pi a}= & -2 m(q+v)\left[\left(q^{+} \cdot q^{-}\right)-2 \frac{(q \cdot v)^{2}}{v^{2}}+m\left(q^{+}\right) m\left(q^{-}\right)\right] \\
& +\left[1+\frac{(q \cdot v)}{v^{2}}\right]\left\{\left(q^{+} \cdot p\right) m\left(q^{-}\right)-\left(q^{-} \cdot p\right) m\left(q^{+}\right)-2(q \cdot v)\left[m\left(q^{+}\right)+m\left(q^{-}\right)\right]\right\}, \\
f_{a a}= & {\left[1+\frac{(q \cdot v)}{v^{2}}\right]\left[q^{+2} q^{-2}-\left(q^{+} \cdot q^{-}\right)(q+v)^{2}-\left(v^{2}+\frac{p^{2}}{4}\right) m\left(q^{+}\right) m\left(q^{-}\right)\right] } \\
& +m(q+v)\left\{m\left(q^{+}\right)\left(q^{-} \cdot p\right)-m\left(q^{-}\right)\left(q^{+} \cdot p\right)\right. \\
& \left.+\frac{(q \cdot v)}{v^{2}}\left(v^{2}-\frac{p^{2}}{4}\right)\left[m\left(q^{+}\right)+m\left(q^{-}\right)\right]\right\}+2 \frac{(q \cdot v)}{v^{2}}(q+v)^{2}\left[(q \cdot v)-\frac{p^{2}}{4}\right] .
\end{aligned}
$$

\subsection{Factores de forma y parámetros del modelo}

Para definir el modelo es necesario especificar los valores de los parámetros y los factores de forma en las corrientes fermiónicas no locales. En este modelo hay seis parámetros a determinar: la masa corriente de quark $m_{c}$, y las constantes de acoplamiento $G_{S}, G_{V}, G_{0}, G_{5}$ y $\varkappa$. Para elegir las formas analíticas de los factores de forma consideraremos los resultados obtenidos en LQCD para el propagador de quarks, que permitirán aproximar su masa dinámica y la función de renormalización de onda, las cuales son funciones del momento como se observa en (3.34). De acuerdo con la Ref. [76], escribimos la masa efectiva $m(p)$ como

$$
m(p)=m_{c}+\alpha_{m} f_{m}\left(p^{2}\right)
$$

donde $\alpha_{m}$ es un parámetro de masa definido por la condición de normalización $f_{m}(0)=1$. Como los cálculos de LQCD consideran diferentes masas corrientes de quark (hay que recordar que $m_{c}$ no es una cantidad física, sino que depende de la escala de renormalización), decidimos tomar como entrada la forma de la función $f_{m}\left(p^{2}\right)$ normalizada, usando los resultados de LQCD en el límite de baja $m_{c}$ y el espaciado de la red más pequeño considerado. Teniendo en cuenta el análisis de LQCD en la Ref. [76], parametrizamos esta función como

$$
f_{m}\left(p^{2}\right)=\frac{1}{1+\left(p^{2} / \Lambda_{0}^{2}\right)^{\alpha}}
$$


con $\alpha=3 / 2$. Por otra parte, para la renormalización de función de onda utilizamos la siguiente parametrización $[63,65]$

$$
z(p)=1-\alpha_{z} f_{z}\left(p^{2}\right)
$$

donde

$$
f_{z}\left(p^{2}\right)=\frac{1}{\left(1+p^{2} / \Lambda_{1}^{2}\right)^{\beta}} .
$$

A partir de esta forma funcional de $z(p)$ los resultados de LQCD resultan mejor descritos con valores relativamente bajos para el exponente $\beta$, por ello tomamos $\beta=5 / 2$ que es el menor exponente compatible con la convergencia ultravioleta de las ecuaciones de gap (3.38). Del análisis dimensional y la invarianza de Lorentz, las funciones $f_{m}\left(p^{2}\right)$ y $f_{z}\left(p^{2}\right)$ deben incluir parámetros dimensionales $\Lambda_{0}$ y $\Lambda_{1}$, los cuales representan cut-offs efectivos para el momento en los canales correspondientes. De este modo, utilizamos las formas funcionales señaladas para los factores de forma, tomando $\Lambda_{0}$ y $\Lambda_{1}$ como dos nuevos parámetros libres del modelo. Por otra parte, los parámetros $\alpha_{m}$ y $\alpha_{z}$ introducidos en las ecuaciones (4.52) y (4.54), en virtud de las expresiones en (3.34), están relacionadas con los valores de expectación de campo medio de los campos escalares a partir de

$$
\begin{aligned}
& m(0)=m_{c}+\alpha_{m}=\frac{m_{c}+\bar{\sigma}_{1}}{1-\bar{\sigma}_{2}}, \\
& z(0)=1-\alpha_{z}=\frac{1}{1-\bar{\sigma}_{2}},
\end{aligned}
$$

y por lo tanto, para un dado conjunto de parámetros, se pueden obtener a partir de las ecuaciones de gap (3.38).

El modelo también incluye los factores de forma $h(p), h_{0}(p)$ y $h_{5}(p)$, introducidos por las interacciones corriente-corriente vectoriales y axiales. Vamos a suponer por simplicidad que el comportamiento efectivo de las interacciones de quark es similar en los canales $J=0$ y $J=1$, y por ello tomaremos para $h(p)$ la misma forma que para $g(p)$. En el sector vectorial-isoescalar, como es usual, supondremos una degeneración aproximada con el sector vectorial-isovectorial tomando $h(p) \simeq h_{0}(p)$. El sector vectorial-isoescalar-axial puede estudiarse separadamente ya que se desacopla del resto de lagrangiano. Aquí tomaremos $h_{5}(p)=h(p)$ y estimaremos el valor resultante para la constante $G_{5}$.

Dadas las formas funcionales de los factores de forma, para estudiar la fenomenología descrita debemos determinar los valores de los ahora ocho parámetros del modelo (masa corriente, constantes de acoplamiento y cut-offs efectivos de momento). Primero llevamos a cabo una aproximación numérica de los resultados de LQCD para las funciones $f_{m}\left(p^{2}\right)$ y $z(p)$, del cual obtenemos valores para $\Lambda_{0}$ y $\Lambda_{1}$, así como para el parámetro $\alpha_{z}$. Éste último, junto con otras cinco cantidades fenomenológicas, será utilizado como valor de entrada 
para determinar los seis parámetros libres restantes. A partir de los resultados de LQCD en Ref. [59] se obtiene

$$
\Lambda_{0}=917 \pm 14 \mathrm{MeV}, \quad \Lambda_{1}=1775 \pm 53 \mathrm{MeV}, \quad \alpha_{z}=0,244 \pm 0,010
$$

con $\chi^{2} /$ dof $=1,17$ y $\chi^{2} /$ dof $=0,25$ para los fits de $f_{m}\left(p^{2}\right)$ y $z(p)$, respectivamente. Los cálculos fueron realizados considerando los resultados de LQCD hasta $2.5 \mathrm{GeV}$. Estos valores y la curva de aproximación para $f_{m}\left(p^{2}\right)$ y $z(p)$ se muestran en la Figura 4.6. En el caso de $z(p)$, se observa que se obtienen valores que superan los puntos de LQCD para momentos bajos. Notamos, sin embargo, que en esta región los errores son relativamente grandes, y además corresponden a los puntos más sensibles a los posibles cambios del espaciado de la red [59].
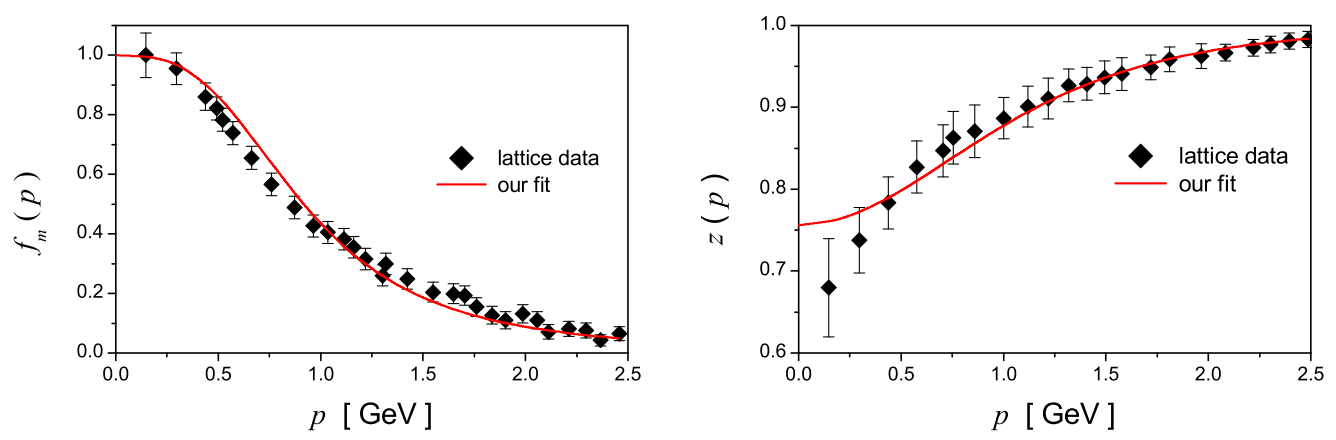

Figura 4.6: Aproximación a los datos de LQCD para las funciones $f_{m}\left(p^{2}\right)$ y $z(p)$.

Ahora bien, una vez fijados los valores para $\Lambda_{0}$ y $\Lambda_{1}$ es posible determinar los parámetros $m_{c}, G_{V}, G_{S}, G_{0}, G_{5}$ y $\varkappa$ a partir de seis cantidades iniciales. Como parámetros de entrada elegimos el valor de $\alpha_{z}$ obtenido a partir del fit anterior, más los valores empíricos de la constante de decaimiento débil del pion $f_{\pi}$, y las masas de los mesones $\pi, \rho$, $\omega$ y $f_{1}$. De nuestro análisis numérico encontramos que se puede obtener un conjunto de parámetros que nos permite reproducir apropiadamente estos valores. Los resultados correspondientes se encuentran en la Tabla 4.1.

El análisis numérico requiere la resolución de un sistema de ecuaciones acopladas que incluye las ecuaciones de gap (3.38), las ecuaciones $G_{M}\left(-m_{M}^{2}\right)=0$ para $M=\pi, \rho, \omega$ y $f_{1}$ para determinar las masas mesónicas, y la ecuación (4.21) para $f_{\pi}$. Este procedimiento involucra calcular las integrales correspondientes a un loop, lo cual en general no es trivial debido al hecho de que el factor de forma $f_{m}\left(p^{2}\right)$, como función de la cuarta componente $p_{4}$ del momento, tiene cortes cuando $p_{4}$ se extiende al campo complejo. Dependiendo del valor del momento tridimensional $\vec{p}$ estos cortes pueden ocasionalmente cruzar el eje 


\begin{tabular}{cc|cccc}
\hline Parámetros & Inputs & \multicolumn{2}{|c}{ Parámetros } & \multicolumn{2}{c}{ Inputs } \\
\hline$\Lambda_{0}[\mathrm{MeV}]$ & fit (LQCD) & $m_{\mathcal{c}}[\mathrm{MeV}]$ & 1,59 & $\alpha_{z}$ & fit (LQCD) \\
$\Lambda_{1}[\mathrm{MeV}]$ & fit (LQCD) & $G_{S} \Lambda_{0}^{2}$ & 19,0 & $m_{\pi}[\mathrm{MeV}]$ & 139 \\
& & $\varkappa / \Lambda_{0}$ & 11,2 & $f_{\pi}[\mathrm{MeV}]$ & 92,2 \\
& & $G_{V} \Lambda_{0}^{2}$ & 13,0 & $m_{\rho}[\mathrm{MeV}]$ & 775 \\
& & $G_{0} \Lambda_{0}^{2}$ & 12,8 & $m_{f_{1}}[\mathrm{MeV}]$ & 1280 \\
& & $G_{5} \Lambda_{0}^{2}$ & $\sim 14$ & $m_{\omega}[\mathrm{MeV}]$ & 783 \\
\hline
\end{tabular}

Tabla 4.1: Parámetros del modelo. Los valores de $\Lambda_{0}, \Lambda_{1}$ y $\alpha_{z}$ se han obtenido a partir de datos de LQCD para el propagador efectivo de los quarks [ecuación (4.58)]. Los parámetros $m_{c}, G_{S}, \varkappa, G_{V}, G_{0}$ y $G_{5}$ fueron calculados a partir de cinco observables hadrónicos, junto con el valor de $\alpha_{z}$.

real, y deben ser tenidos en cuenta a través de deformaciones adecuadas del camino de integración. Estos cálculos se encuentran detallados en el Apéndice B.

De la Tabla 4.1 encontramos una relación $G_{S} / G_{V} \sim 1,5$, lo cual está de acuerdo con las parametrizaciones estándar del modelo NJL [26, 27, 28]. Para el valor $G_{0}$, es necesario tener en cuenta que estamos trabajando en un modelo con sólo dos sabores de quarks, y por lo tanto no estamos considerando efectos relacionados con el quark $s$. Nuestro cálculo para $G_{0}$ sería válido únicamente en el caso de una mezcla ideal entre el singulete de $\mathrm{SU}(3)_{f}$ y el octete de estados $I=0$, tal que el mesón $\phi$ sea un estado $\bar{s} s$ aproximadamente puro. En el caso del mesón axial $f_{1}$ encontramos una dificultad adicional, que es común a varios modelos de quarks. Los modelos que no incluyen un mecanismo explícito para el confinamiento usualmente tienen dificultades para describir las resonancias mesónicas, ya que hay un umbral a partir del cual la masa del mesón es lo suficientemente grande como para permitir su decaimiento en dos quarks. Este umbral es en general del orden de $2 m(0)$, por lo tanto los modelos para los que las masas constituyentes de quarks son mayores que aproximadamente $400 \mathrm{MeV}$ (como ocurre en nuestro caso) pueden sortear esta dificultad para resonancias de baja masa como el mesón $\rho$ [77]. En el caso en que la resonancia supera el umbral, es posible tratar el problema a través de extensiones de las funciones $G_{M}(-s)$ al plano complejo [78], o de la búsqueda de un pico en la función espectral del mesón [79]. Matemáticamente, en nuestro modelo la apertura del canal no físico $q \bar{q}$ ocurre debido a que las integrales de la forma de (4.10) tienen un "pinch point" en el cual ambas funciones $D\left(q^{+}\right)$y $D\left(q^{-}\right)$en el integrando son iguales a cero (es decir, ambos quarks constituyentes están simultáneamente on-shell). Para los parámetros en la Tabla 4.1, el umbral se encuentra a los $1264 \mathrm{MeV}$, debajo del valor empírico $m_{f_{1}} \simeq 1280 \mathrm{MeV}$, y el parámetro libre a ser ajustado para obtener el valor fenomenológico de la masa de $f_{1}$ es la 
constante de acoplamiento $G_{5}$. En nuestro trabajo, para obtener un valor aproximado para esta constante resolvemos la ecuación $G_{f_{1}}\left(-m^{2}\right)=0$ variando $G_{5} \Lambda_{0}^{2}$ desde grandes valores de $G_{5}$ hasta $G_{5} \Lambda_{0}^{2} \simeq 22$, lo que lleva hasta $m \simeq 1 \mathrm{GeV}$, y luego extrapolamos a la región por arriba del umbral obteniendo $m_{f_{1}} \simeq 1280 \mathrm{MeV}$ para $G_{5} \Lambda_{0}^{2} \sim 14$.

\subsection{Resultados numéricos}

Usando los parámetros y los factores de forma no locales definidos en la sección anterior, podemos calcular las predicciones del modelo para las cantidades fenomenológicas analizadas. Nuestros resultados para dichos observables se encuentran resumidos en la Tabla 4.2 (no incluimos allí los observables que utilizamos como entrada para fijar la parametrización, es decir, $m_{\pi}, f_{\pi}, m_{\rho}, m_{\omega}$ y $m_{f_{1}}$ ). De la tabla podemos concluir que las predicciones del modelo para los anchos de decaimiento $\pi^{0} \rightarrow \gamma \gamma, \rho \rightarrow e^{+} e^{-}$y $\rho \rightarrow \pi \pi$ están de acuerdo con los valores encontrados experimentalmente, siendo compatibles con los resultados publicados por el Particle Data Group [75] dentro de un rango de error menor al $10 \%$. También fue posible obtener una predicción para el ancho de decaimiento $\Gamma\left(\omega \rightarrow e^{+} e^{-}\right)$,

\begin{tabular}{c|c|c}
\hline & Modelo & Empírico \\
\hline$\Gamma\left(\pi^{0} \rightarrow \gamma \gamma\right)[\mathrm{MeV}]$ & $7,82 \times 10^{-6}$ & $(7,63 \pm 0,16) \times 10^{-6}$ \\
$\Gamma\left(\rho \rightarrow e^{+} e^{-}\right)[\mathrm{MeV}]$ & $6,71 \times 10^{-3}$ & $(7,04 \pm 0,06) \times 10^{-3}$ \\
$\Gamma(\rho \rightarrow \pi \pi)[\mathrm{MeV}]$ & 137 & $149,1 \pm 0,8$ \\
$m_{\sigma}[\mathrm{MeV}]$ & 683 & $400-550$ \\
$m_{\mathrm{a}_{1}}[\mathrm{MeV}]$ & $1200-1250$ & $1190-1270$
\end{tabular}

Tabla 4.2: Predicciones del modelo y valores empíricos [75] para varios observables.

cuyo valor determinamos alrededor de $0,8 \mathrm{keV}$, algo mayor que el valor experimental de $0,60 \pm 0,02 \mathrm{keV}$ [75]. Sin embargo, como discutimos anteriormente, nuestro resultado podría modificarse luego de incluir grados de libertad de extrañeza debido a la mezcla $\omega-\phi$. Con respecto al sector $\sigma-\sigma^{\prime}$, obtuvimos un estado físico con una masa de alrededor de 680 $\mathrm{MeV}$, el cual puede identificarse con la resonancia del mesón $\sigma$ observada (cuya masa es incierta), mientras que para $\sigma^{\prime}$ encontramos un crecimiento monótono de la función $G_{\sigma^{\prime}}(-s)$ con $s$, indicando que este estado no representa una partícula física (en la Ref. [63] se discute con mayor profundidad la física relacionada al estado $\sigma^{\prime}$ en este tipo de modelos).

En el caso de los mesones axiales a encontramos que la función $G_{\mathrm{a}_{1}}(-s)$ decrece con $s$ hasta llegar a un mínimo en $\sqrt{s} \simeq 1250 \mathrm{MeV}$, cerca del umbral de producción de pares de quarks on-shell, encontrado en $1264 \mathrm{MeV}$. Teniendo en cuenta la discusión en la subsección 
anterior, para estimar el valor de la masa del $\mathrm{a}_{1}$ en posible tomar el mínimo de $G_{\mathrm{a}_{1}}(-s)$ o realizar una extrapolación basada en el comportamiento de $G_{\mathrm{a}_{1}}(-s)$ hasta $s \sim(1 \mathrm{GeV})^{2}$. Ambos caminos conducen a una masa aproximada de $m_{\mathrm{a}_{1}} \sim 1200-1250 \mathrm{MeV}$, que está en buen acuerdo con los valores experimentales.

También analizamos la dependencia de nuestros resultados con $\alpha_{z}$ dentro del error obtenido para el cálculo de este parámetro en la aproximación a los datos de LQCD [ecuación (4.58)], observando que las predicciones del modelo no varían significativamente.

Finalmente, en la Tabla 4.3 mostramos los resultados para los valores de campo medio de los campos escalares y los condensados quirales de quark. La masa efectiva de los quarks livianos a momento cero en nuestro modelo resulta $m(0)=\left(m_{c}+\bar{\sigma}_{1}\right) /\left(1-\bar{\sigma}_{2}\right) \simeq$ $400 \mathrm{MeV}$, algo mayor que los $311 \mathrm{MeV}$ obtenidos en la Ref. [63] para un modelo nlNJL sin el sector de los grados de libertad vectoriales. Cabe destacar que las parametrizaciones de los modelos NJL estándar predicen masas constituyentes de quarks dependientes del momento que rondan los $350 \mathrm{MeV}$ [26, 27, 28]. Con respecto a los condensados de quarks, los resultados son relativamente grandes en comparación con las estimaciones usuales de la fenomenología y los cálculos de LQCD, que conducen a condensados dentro del rango de $(-240 \mathrm{MeV})^{3}$ a $(-320 \mathrm{MeV})^{3}$ [80]. Además, al determinar los parámetros del modelo hemos encontrado un valor relativamente bajo para la masa corriente de los quarks, siendo $m_{c}=1,59 \mathrm{MeV}$, en comparación con las estimaciones de LQCD de $m_{c} \simeq 3,4 \pm 0,25 \mathrm{MeV}$ en el límite de isospín [75]. En los modelos NJL con interacciones no locales estas cantidades dependen fuertemente de las formas funcionales de los factores de forma, como se explica en las Refs. [63, 81, 82], en donde se consideran distintas extensiones de estos modelos con dos o tres sabores de quarks, sin el sector vectorial. Como se discute en esos artículos, es importante tener en cuenta la dependencia de $m_{c} \mathrm{y}\langle\bar{q} q\rangle$ con la escala de renormalización. En nuestro caso, para el fit de los propagadores de quarks se utilizaron datos de LQCD correspondientes a una escala $\mu=3 \mathrm{GeV}$, mayor que la usual de $2 \mathrm{GeV}$. Para evitar este tipo de dependencias se puede, por ejemplo, calcular el producto $-\langle\bar{q} q\rangle m_{c}$, para el cual obtenemos, dentro de nuestra parametrización, un resultado de $8,12 \times 10^{-5} \mathrm{GeV}^{4}$. Este valor concuerda con el proveniente de la relación de Gell-Mann-Oakes-Renner a primer orden en la expansión quiral, $-\langle\bar{q} q\rangle m_{c}=f_{\pi}^{2} m_{\pi}^{2} / 2 \simeq 8,21 \times 10^{-5} \mathrm{GeV}^{4}$.

\begin{tabular}{c|c}
\hline & Modelo \\
\hline $\bar{\sigma}_{1}[\mathrm{MeV}]$ & 524 \\
$\bar{\sigma}_{2}$ & -0.322 \\
$-\langle\bar{q} q\rangle^{1 / 3}[\mathrm{MeV}]$ & 371
\end{tabular}

Tabla 4.3: Resultados numéricos para varias cantidades fenomenológicas. 



\section{Capítulo 5}

\section{Modelo NJL no local en presencia de campo magnético}

Como se ha comentado en el Cap. 1, el comportamiento de la materia hadrónica y de quarks en presencia de campos magnéticos intensos, y los efectos de estos campos sobre el diagrama de fases, revisten importante interés teórico y experimental. En este capítulo estudiaremos la interacción entre la materia hadrónica y un campo magnético homogéneo en un modelo NJL no local. En primer lugar, calcularemos el determinante fermiónico en la acción bosonizada y realizaremos una aproximación de campo medio. Esto nos permitirá encontrar expresiones analíticas para la ecuación del gap, el propagador de quarks en presencia de campo magnético y el condensado quiral. Luego, desarrollando la acción a segundo orden en las fluctuaciones de los campos mesónicos calcularemos las masas de los mesones $\pi$ y $\sigma$, e incorporando un acoplamiento con una corriente externa obtendremos la expresión para la constante de decaimiento débil del pion. También mostraremos dentro de nuestro modelo la validez de las relaciones de Goldberger-Treiman y de Gell-Mann-Oakes-Renner en presencia del campo magnético. Por último, mostraremos los resultados numéricos obtenidos para las cantidades fenomenológicas mencionadas considerando diferentes parametrizaciones del modelo, y compararemos estos resultados con los obtenidos en LQCD.

\subsection{Interacciones magnéticas en el modelo NJL no local}

Es natural que la presencia de interacciones con campos magnéticos intensos afecte significativamente a los observables hadrónicos y a las características de las transiciones de fase. Es de particular interés el estudio de los condensados quirales $\langle\bar{q} q\rangle$, parámetros de orden de la restauración de la simetría quiral. Cálculos realizados por LQCD [83, 84] 
muestran que, en efecto, los condensados quirales tanto en el vacío como en sistemas con temperatura finita resultan fuertemente alterados en presencia de una interacción con un campo magnético externo $B$ uniforme suficientemente intenso. En el vacío se observa el efecto conocido como "catálisis magnética", esto es, la presencia del campo favorece la presencia de un condensado no nulo, y de este modo puede interpretarse como un "catalizador" de la transición de fase. Asimismo, los cálculos realizados en LQCD muestran un efecto de "catálisis magnética inversa", que consiste en el hecho de que las temperaturas críticas de transición disminuyen al incrementarse el valor del campo externo. Notablemente, este efecto no es reproducido en general por modelos efectivos simples para QCD a bajas energías, como el modelo NJL local, $\chi$ PT, o el "modelo de la bolsa del MIT" (MIT Bag model), que de hecho predicen el efecto contrario [85, 86, 87]. En el caso del modelo NJL local, una posible solución para este problema se obtiene proponiendo una dependencia ad-hoc con $B$ y $T$ en las constantes de acoplamiento [88, 89].

En esta Tesis realizaremos un análisis detallado del modelo NJL no local que incluye el acoplamiento magnético con un campo $B$ uniforme y constante [90, 91]. Mostraremos que los modelos NJL no locales no sólo proveen una descripción natural para los efectos de la catálisis magnética y la catálisis magnética inversa, sino que también permiten obtener una dependencia de la masa del $\pi^{0}$ con $B$ que resulta compatible con los resultados de LQCD $[92,93]$. El estudio de la variación de las masas de los piones en presencia de un campo magnético ha sido abordado en los últimos años en el marco de diversas técnicas teóricas para el análisis de QCD a bajas energías, como modelos de tipo NJL [94, 95, 96, 97],

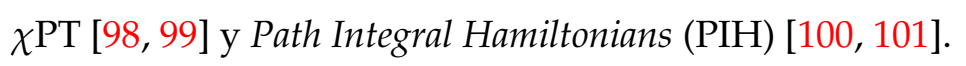

\subsubsection{Formalismo en la aproximación de campo medio}

Para estudiar las interacciones magnéticas comenzamos escribiendo la acción euclídea del modelo NJL no local para dos sabores de quarks,

$$
S_{E}=\int d^{4} x\left\{\bar{\psi}(x)\left(-i \not \supset+m_{c}\right) \psi(x)-\frac{G}{2} j_{a}(x) j_{a}(x)\right\}
$$

donde las corrientes no locales $j_{a}(x)$ están dadas por

$$
j_{a}(x)=\int d^{4} z \mathcal{G}(z) \bar{\psi}\left(x+\frac{z}{2}\right) \Gamma_{a} \psi\left(x-\frac{z}{2}\right) .
$$

Definimos aquí $\Gamma_{a}=\left(\mathbb{1}, i \gamma_{5} \vec{\tau}\right)$, mientras que la función $\mathcal{G}(z)$ corresponde al factor de forma no local que caracteriza la interacción efectiva. Como estamos interesados en estudiar la influencia del campo magnético, introducimos en la acción efectiva un acoplamiento con un campo de gauge electromagnético externo $\mathcal{A}_{\mu}$. Esto se lleva a cabo de forma análoga a lo realizado en el Cap. 4 para estudiar el vértice $\rho$-fotón, es decir, se reemplaza la derivada 
covariante de acuerdo a la ecuación (3.47), y se introduce el campo electromagnético según (4.24). Como también se discutió anteriormente, las transformaciones de gauge deben incluirse en las corrientes no locales a través del transporte paralelo que se observa en las ecuaciones (3.48).

Luego procedemos a realizar la bosonización de la teoría introduciendo el campo escalar $\sigma(x)$ y los campos pseudoescalares $\vec{\pi}(x)$, e integrando los campos fermiónicos en la función de partición de acuerdo con los lineamientos explicados en el Apéndice A. La acción bosonizada resulta $[63,69]$

$$
S_{\mathrm{bos}}=-\log \operatorname{det} \mathcal{D}_{x, x^{\prime}}+\frac{1}{2 G} \int d^{4} x[\sigma(x) \sigma(x)+\vec{\pi}(x) \cdot \vec{\pi}(x)],
$$

con

$$
\begin{aligned}
\mathcal{D}_{x, x^{\prime}}= & \delta^{(4)}\left(x-x^{\prime}\right)\left(-i \not D+m_{c}\right) \\
& +\mathcal{G}\left(x-x^{\prime}\right) \gamma_{0} \mathcal{W}(x, \bar{x}) \gamma_{0}\left[\sigma(\bar{x})+i \gamma_{5} \vec{\tau} \cdot \vec{\pi}(\bar{x})\right] \mathcal{W}\left(\bar{x}, x^{\prime}\right)
\end{aligned}
$$

donde hemos definido $\bar{x}=\left(x+x^{\prime}\right) / 2$ y las funciones $\mathcal{W}(x, \bar{x})$ son las definidas en (3.49). El operador $\mathcal{D}_{x, x^{\prime}}$ es análogo al que hemos definido en los Caps. 3 y 4 como $A(p, p \prime)$ en ausencia del campo magnético. Consideraremos en este análisis el caso particular de un campo magnético $\vec{B}$ homogéneo y constante en el tiempo, eligiendo el eje 3 en la dirección del campo. Utilizaremos el gauge de Landau, en el que $\mathcal{A}_{\mu}=B x_{1} \delta_{\mu 2}$.

Supondremos también que el campo $\sigma(x)$ tiene un valor de expectación de vacío no trivial invariante traslacional (es decir, que $\bar{\sigma}$ es independiente de $x$ ), mientras que los valores de campo medio de los campos pseudoescalares $\pi_{i}$ son nulos. Esta hipótesis no implica que el propagador de quark resultante sea invariante traslacional. De hecho, veremos a partir de nuestro análisis que esta invarianza se rompe por la aparición de una "fase de Schwinger". A partir de (5.3), dentro de la aproximación de campo medio (MFA) obtenemos

$$
\mathcal{D}_{x, x^{\prime}}^{\mathrm{MFA}}=\operatorname{diag}\left(\mathcal{D}_{x, x^{\prime}}^{\mathrm{MFA}, u}, \mathcal{D}_{x, x^{\prime}}^{\mathrm{MFA}, d}\right)
$$

donde

$$
\mathcal{D}_{x, x^{\prime}}^{\mathrm{MFA}, f}=\delta^{(4)}\left(x-x^{\prime}\right)\left(\Pi^{f}+m_{c}\right)+\bar{\sigma} \mathcal{G}\left(x-x^{\prime}\right) \exp \left[i \Phi_{f}\left(x, x^{\prime}\right)\right],
$$

sobreentendiendo un producto directo por una matriz identidad en el espacio de color. En la ecuación (5.6) hemos introducido el operador $\Pi^{f}=-i \not \supset-q_{f} B x_{1} \gamma_{2}$ junto con la denominada "fase de Schwinger"

$$
\Phi_{f}\left(x, x^{\prime}\right)=\frac{q_{f} B}{2}\left(x_{2}-x_{2}^{\prime}\right)\left(x_{1}+x_{1}^{\prime}\right) .
$$

Debido a que los quarks $u$ y $d$ tienen cargas eléctricas diferentes, el campo magnético interactúa de distinto modo con cada uno de ellos. Por lo tanto, este modelo no es simétrico 
en el espacio de sabor, lo cual se refleja en la dependencia con $q_{f}$ que representa las cargas eléctricas de cada sabor de quark provenientes de la matriz de carga $\hat{Q}$ que se introduce en el acoplamiento electromagnético.

De este modo, la acción bosonizada en MFA por unidad de volumen puede escribirse como

$$
\frac{S_{\mathrm{bos}}^{\mathrm{MFA}}}{V^{(4)}}=\frac{\bar{\sigma}^{2}}{2 G}-\frac{N_{c}}{V^{(4)}} \sum_{f=u, d} \operatorname{tr} \log \mathcal{D}_{x, x^{\prime}}^{\mathrm{MFA}, f}
$$

donde en el segundo término las trazas sobre color y sabor ya han sido evaluadas. Para realizar las trazas en el espacio de coordenadas y de Dirac utilizaremos el formalismo de las autofunciones de Ritus [102], realizando la transformada de Ritus de $\mathcal{D}_{x, x^{\prime}}^{\mathrm{MFA}, f}$. Ésta se define como

$$
\mathcal{D}_{\bar{p}, \bar{p}^{\prime}}^{\mathrm{MFA}, f}=\int d^{4} x d^{4} x^{\prime} \overline{\mathbb{E}}_{\bar{p}}(x) \mathcal{D}_{x, x^{\prime}}^{\mathrm{MFA}, f} \mathbb{E}_{\bar{p}^{\prime}}\left(x^{\prime}\right),
$$

donde $\mathbb{E}_{\bar{p}}(x)$, con $\bar{p}=\left(k, p_{2}, p_{3}, p_{4}\right)$ (siendo $k$ un valor discreto), son las funciones de Ritus, cuyas definiciones y propiedades están dadas en el Apéndice C. Usando estas propiedades obtenemos

$$
\mathcal{D}_{\bar{p}, \bar{p}^{\prime}}^{\mathrm{MFA}, f}=\hat{\delta}_{\bar{p}, \bar{p}^{\prime}} P_{k, s_{f}}\left(-s_{f} \sqrt{2 k\left|q_{f} B\right|} \gamma_{2}+p_{\|} \cdot \gamma_{\|}+m_{c} \mathbb{1}\right)+\bar{\sigma} \sum_{\lambda= \pm} G_{\bar{p}, \bar{p}^{\prime}}^{\lambda, f} \Delta^{\lambda}
$$

donde $\hat{\delta}_{\bar{p}, \bar{p}^{\prime}}$ es una notación para $(2 \pi)^{4} \delta_{k k^{\prime}} \delta\left(p_{2}-p_{2}^{\prime}\right) \delta\left(p_{3}-p_{3}^{\prime}\right) \delta\left(p_{4}-p_{4}^{\prime}\right)$, y hemos introducido las definiciones $s_{f}=\operatorname{signo}\left(q_{f} B\right), p_{\|}=\left(p_{3}, p_{4}\right), \gamma_{\|}=\left(\gamma_{3}, \gamma_{4}\right), \Delta^{+}=\operatorname{diag}(1,0,1,0)$, $\Delta^{-}=\operatorname{diag}(0,1,0,1)$ y $P_{k, \pm 1}=\left(1-\delta_{k 0}\right) \mathbb{1}+\delta_{k 0} \Delta^{ \pm}$. Las funciones $G_{\bar{p}, \bar{p}^{\prime}}^{\lambda, f}$ están dadas por

$$
G_{\bar{p}, \bar{p}^{\prime}}^{\lambda, f}=\int d^{4} x d^{4} x^{\prime} E_{\bar{p} \lambda}^{*}(x) \mathcal{G}\left(x-x^{\prime}\right) \exp \left[i \Phi_{f}\left(x, x^{\prime}\right)\right] E_{\bar{p}^{\prime} \lambda}\left(x^{\prime}\right),
$$

y la expresión de $E_{\bar{p} \lambda}(x)$ viene dada por la ecuación (C4). Tal como se discute en el Apéndice D, luego de un largo cálculo podemos mostrar que $G_{\bar{p}, \bar{p}^{\prime}}^{\lambda, f}$ es diagonal en $\bar{p}, \bar{p}^{\prime}$. Obtenemos entonces $G_{\bar{p}, \bar{p}^{\prime}}^{\lambda, f}=\hat{\delta}_{\bar{p}, \bar{p}^{\prime}} g_{k, p_{\|}}^{\lambda, f}$, donde

$$
g_{k, p_{\|}}^{\lambda, f}=\frac{4 \pi}{\left|q_{f} B\right|}(-1)^{k_{\lambda}} \int \frac{d^{2} p_{\perp}}{(2 \pi)^{2}} g\left(p_{\perp}^{2}+p_{\|}^{2}\right) \exp \left(-\frac{p_{\perp}^{2}}{\left|q_{f} B\right|}\right) L_{k_{\lambda}}\left(\frac{2 p_{\perp}^{2}}{\left|q_{f} B\right|}\right) .
$$

Aquí hemos utilizado las definiciones $k_{ \pm}=k-1 / 2 \pm s_{f} / 2$ y $p_{\perp}=\left(p_{1}, p_{2}\right)$, mientras que $g\left(p^{2}\right)$ es la transformada de Fourier de $\mathcal{G}(x)$ y $L_{m}(x)$ son los polinomios de Laguerre, con la convención usual $L_{-1}(x)=0$. Definiendo ahora

$$
M_{k, p_{\|}}^{\lambda, f}=\left(1-\delta_{k_{\lambda},-1}\right) m_{c}+\bar{\sigma} g_{k, p_{\|}}^{\lambda, f},
$$

llegamos a la expresión $\mathcal{D}_{\bar{p}, \bar{p}^{\prime}}^{\mathrm{MFA}, f}=\hat{\delta}_{\bar{p}, \bar{p}} \mathcal{D}_{k, p_{\|}}^{f}$, donde

$$
\mathcal{D}_{k, p_{\|}}^{f}=P_{k, s_{f}}\left(-s_{f} \sqrt{2 k\left|q_{f} B\right|} \gamma_{2}+p_{\|} \cdot \gamma_{\|}\right)+\sum_{\lambda= \pm} M_{k, p_{\|}}^{\lambda, f} \Delta^{\lambda} .
$$


Luego, usando la ecuación (C16) y escribiendo explícitamente la traza sobre el espacio de coordenadas obtenemos

$$
\operatorname{tr} \log \mathcal{D}_{x, x^{\prime}}^{\mathrm{MFA}, f}=\frac{N_{c}}{2 \pi} \int d^{4} x \sum_{k=0}^{\infty} \frac{d^{2} p_{\|}}{(2 \pi)^{2}} \int_{-\infty}^{\infty} \frac{d p_{2}}{2 \pi} \operatorname{tr}_{D}\left[\mathbb{E}_{\bar{p}}(x) \log \left(\mathcal{D}_{k, p_{\|}}^{f}\right) \overline{\mathbb{E}}_{\bar{p}}(x)\right],
$$

donde $\operatorname{tr}_{D}$ es la traza sobre el espacio de Dirac. A partir de la propiedad cíclica de la traza, junto con la ecuación (C9), esta expresión se reduce a

$$
\operatorname{tr} \log \mathcal{D}_{x, x^{\prime}}^{\mathrm{MFA}, f}=V^{(4)} N_{c} \frac{\left|q_{f} B\right|}{2 \pi} \sum_{k=0}^{\infty} \int \frac{d^{2} p_{\|}}{(2 \pi)^{2}} \operatorname{tr}_{D}\left[P_{k, s_{f}} \log \left(\mathcal{D}_{k, p_{\|}}^{f}\right)\right] .
$$

Como la matriz $\mathcal{D}_{k, p \|}^{f}$ no es diagonal en el espacio de Dirac, es conveniente usar aquí la identidad $\operatorname{tr} \log A=\log \operatorname{det} A$. Luego de calcular el determinante y reemplazar en la ecuación (5.8) obtenemos finalmente

$$
\frac{S_{\mathrm{bos}}^{\mathrm{MFA}}}{V^{(4)}}=\frac{\bar{\sigma}^{2}}{2 G}-N_{c} \sum_{f=u, d} \frac{\left|q_{f} B\right|}{2 \pi} \int \frac{d^{2} p_{\|}}{(2 \pi)^{2}}\left[\log \left(p_{\|}^{2}+M_{0, p_{\|}}^{\lambda, f^{2}}\right)+\sum_{k=1}^{\infty} \log \Delta_{k, p_{\|}}^{f}\right],
$$

donde $\lambda=+(-)$ para $s_{f}=+1(-1), y \Delta_{k, p_{\|}}^{f}$ está definida por

$$
\Delta_{k, p_{\|}}^{f}=\left(2 k\left|q_{f} B\right|+p_{\|}^{2}+M_{k, p_{\|}}^{+, f} M_{k, p_{\|}}^{-, f}\right)^{2}+p_{\|}^{2}\left(M_{k, p_{\|}}^{+, f}-M_{k, p_{\|}}^{-, f}\right)^{2}
$$

Se observa que las funciones $M_{k, p_{\|}}^{ \pm, f}$ representan las masas efectivas dinámicas (dependientes del momento) de los quarks en presencia del campo magnético externo. El valor de expectación de vacío $\bar{\sigma}$ puede calcularse ahora encontrando el mínimo de la acción efectiva en la ecuación (5.17). Esto lleva a la ecuación de gap

$$
\frac{\bar{\sigma}}{G}=N_{c} \sum_{f=u, d} \frac{\left|q_{f} B\right|}{\pi} \sum_{k=0}^{\infty} \int \frac{d^{2} p_{\|}}{(2 \pi)^{2}} \sum_{\lambda= \pm} \hat{A}_{k, p_{\|}}^{\lambda, f} g_{k, p_{\|}}^{\lambda, f}
$$

donde hemos definido

$$
\hat{A}_{k, p_{\|}}^{ \pm, f}=\frac{M_{k, p_{\|}}^{\mp, f}\left(2 k\left|q_{f} B\right|+p_{\|}^{2}+M_{k, p_{\|}}^{-, f} M_{k, p_{\|}}^{+, f}\right)+p_{\|}^{2}\left(M_{k, p_{\|}}^{ \pm, f}-M_{k, p_{\|}}^{\mp, f}\right)}{\Delta_{k, p_{\|}}^{f}} .
$$

Asimismo, a partir de la expresión en (5.14), se pueden obtener los propagadores de quark en MFA. Los detalles de este cálculo están dados en el Apéndice E. En el espacio de coordenadas obtenemos [91]

$$
S_{x, x^{\prime}}^{\mathrm{MFA}, f}=\left(\mathcal{D}_{x, x^{\prime}}^{\mathrm{MFA}, f}\right)^{-1}=\exp \left[i \Phi_{f}\left(x, x^{\prime}\right)\right] \int \frac{d^{4} p}{(2 \pi)^{4}} e^{i p \cdot\left(x-x^{\prime}\right)} \tilde{S}^{f}\left(p_{\perp}, p_{\|}\right),
$$


donde

$$
\begin{aligned}
\tilde{S}^{f}\left(p_{\perp,} p_{\|}\right)= & 2 \exp \left(-\frac{p_{\perp}^{2}}{\left|q_{f} B\right|}\right) \sum_{k=0}^{\infty} \sum_{\lambda= \pm}\left[(-1)^{k_{\lambda}}\left(\hat{A}_{k, p_{\|}}^{\lambda, f}-\hat{B}_{k, p_{\|}}^{\lambda, f} p_{\|} \cdot \gamma_{\|}\right) L_{k_{\lambda}}\left(\frac{2 p_{\perp}^{2}}{\left|q_{f} B\right|}\right)\right. \\
& \left.+2(-1)^{k}\left(\hat{C}_{k, p_{\|}}^{\lambda, f}-\hat{D}_{k, p_{\|}}^{\lambda, f} p_{\|} \cdot \gamma_{\|}\right) p_{\perp} \cdot \gamma_{\perp} L_{k-1}^{1}\left(\frac{2 p_{\perp}^{2}}{\left|q_{f} B\right|}\right)\right] \Delta^{\lambda}
\end{aligned}
$$

Aquí hemos introducidos las definiciones

$$
\begin{aligned}
\hat{B}_{k, p_{\|}}^{ \pm, f} & =\hat{C}_{k, p_{\|}}^{ \pm, f}-M_{k, p_{\|}}^{\mp, f} \hat{D}_{k, p_{\|}}^{ \pm, f}, \\
\hat{C}_{k, p_{\|}}^{ \pm, f} & =\frac{2 k\left|q_{f} B\right|+p_{\|}^{2}+M_{k, p_{\|}}^{-, f} M_{k, p_{\|}}^{+, f}}{\Delta_{k, p_{\|}}^{f}} \\
\hat{D}_{k, p_{\|}}^{ \pm, f} & =\frac{M_{k, p_{\|}}^{ \pm, f}-M_{k, p_{\|}}^{\mp, f}}{\Delta_{k, p_{\|}}^{f}}
\end{aligned}
$$

mientras que las funciones $L_{k}^{1}(x)$ son los polinomios de Laguerre generalizados, con $L_{-1}^{1}=$ 0 . A partir de estas definiciones, las funciones $\hat{A}_{k, p \|}^{\lambda, f}$ en (5.20) satisfacen

$$
\hat{A}_{k, p_{\|}}^{ \pm, f}=M_{k, p_{\|}}^{\mp, f} \hat{C}_{k, p_{\|}}^{ \pm, f}+p_{\|}^{2} \hat{D}_{k, p_{\|}}^{ \pm, f} .
$$

De este modo, hemos obtenido que los propagadores de quark pueden escribirse como el producto de una exponencial de la fase de Schwinger por una función invariante traslacional, en acuerdo con los análisis llevados a cabo en la Ref. [103].

Una vez obtenida la expresión para los propagadores, los condensados de quark para cada sabor pueden calcularse fácilmente a partir de la relación

$$
\left\langle\bar{q}_{f} q_{f}\right\rangle=-N_{c} \operatorname{tr}_{D}\left[S_{x, x}^{\mathrm{MFA}, f}\right] .
$$

A partir de este cálculo se obtiene ${ }^{1}$

$$
\begin{aligned}
\left\langle\bar{q}_{f} q_{f}\right\rangle & =-\frac{1}{V^{(4)}} \operatorname{Tr} S_{f}^{\mathrm{MFA}}=-N_{C} \int \frac{d^{4} p}{(2 \pi)^{4}} \operatorname{tr}_{D} \tilde{S}_{f}\left(p_{\perp}, p_{\|}\right) \\
& =-4 \sum_{k=0}^{\infty} \int_{q_{\|}} \sum_{\lambda= \pm}(-1)^{k_{\lambda}} \hat{A}_{k, p_{\|}}^{\lambda, f} \int \frac{d^{2} p_{\perp}}{(2 \pi)^{2}} \exp \left(-\frac{p_{\perp}^{2}}{\left|q_{f} B\right|}\right) L_{k_{\lambda}}\left(\frac{2 p_{\perp}^{2}}{\left|q_{f} B\right|}\right) \\
& =-\frac{N_{C}\left|q_{f} B\right|}{\pi} \sum_{k=0}^{\infty} \int_{q_{\|}} \sum_{\lambda= \pm} \hat{A}_{k, q_{\|}}^{\lambda, f} .
\end{aligned}
$$

Del mismo modo que en el Cap. 3 la integral en el condensando quiral resulta ser divergente y es por eso necesario regularizarlo. Siguiendo la misma prescripción se define

$$
\left\langle\bar{q}_{f} q_{f}\right\rangle_{B}^{\mathrm{reg}}=\left\langle\bar{q}_{f} q_{f}\right\rangle-\left\langle\bar{q}_{f} q_{f}\right\rangle^{\text {free }}+\left\langle\bar{q}_{f} q_{f}\right\rangle_{\text {free }}^{\text {reg }} .
$$

\footnotetext{
${ }^{1}$ Otra forma de determinar los condensados es a través de la derivada de $S^{\mathrm{MFA}}$ con respecto a la masa corriente de los quarks.
} 
Por contribución "libre" se entiende aquí al condensado en ausencia de el acoplamiento efectivo de cuatro fermiones, pero manteniendo la interacción con el campo magnético. Esta contribución puede regularizarse obteniendo [50, 91, 104]

$$
\left\langle\bar{q}_{f} q_{f}\right\rangle_{\text {free }}^{\mathrm{reg}}=\frac{N_{C} m_{c}^{3}}{4 \pi^{2}}\left[\frac{\log \Gamma\left(x_{f}\right)}{x_{f}}-\frac{\log 2 \pi}{2 x_{f}}+1-\left(1-\frac{1}{2 x_{f}}\right) \log x_{f}\right],
$$

donde $x_{f}=m_{c}^{2} /\left(2\left|q_{f} B\right|\right)$.

Finalmente, para comparar con los resultados de LQCD presentados en la Ref. [84] definimos el "condensado normalizado" $\Sigma_{B}^{f}$ según

$$
\Sigma_{B}^{f}=-\frac{2 m_{c}}{S^{4}}\left[\left\langle\bar{q}_{f} q_{f}\right\rangle_{B}^{\mathrm{reg}}-\left\langle\bar{q}_{f} q_{f}\right\rangle_{0}^{\mathrm{reg}}\right]+1
$$

donde $S$ es una escala fenomenológica fijada en $S=(135 \times 86)^{1 / 2} \mathrm{MeV}$. También introducimos las definiciones $\Delta \Sigma_{B}^{f}=\Sigma_{B}^{f}-\Sigma_{0}^{f}$ y $\Delta \bar{\Sigma}_{B}=\left(\Delta \Sigma_{B}^{u}+\Delta \Sigma_{B}^{d}\right) / 2$.

\subsubsection{Constantes fenomenológicas para los mesones $\sigma$ y $\pi^{0}$}

\section{Masas del sigma y del pion}

La expresión del propagador de quark (5.21) puede utilizarse de modo de obtener expresiones analíticas para las masas de los mesones $\pi^{0}$ y $\sigma$ en presencia del campo magnético uniforme $\vec{B}$. Primero nos concentraremos en la masa del $\pi^{0}$, la cual surge de los términos en la expansión de la acción bosonizada $S_{\text {bos }}$ que son cuadráticos en $\delta \pi_{3}$. Desarrollando el primer término en el lado derecho de la ecuación (5.3) alrededor de los valores medios de los campos mesónicos obtenemos

$$
\begin{aligned}
-\log \operatorname{det} \mathcal{D} & =-\operatorname{Tr} \log \mathcal{D}_{0}-\operatorname{Tr} \log \left(1+\mathcal{D}_{0}^{-1} \delta \mathcal{D}\right) \\
& =-\operatorname{Tr} \log \mathcal{D}_{0}-\operatorname{Tr}\left(\mathcal{D}_{0}^{-1} \delta \mathcal{D}\right)+\frac{1}{2} \operatorname{Tr}\left(\mathcal{D}_{0}^{-1} \delta \mathcal{D}\right)^{2}+\ldots
\end{aligned}
$$

De este modo, el desarrollo a orden cuadrático de la ecuación (5.4) en las fluctuaciones del campo $\delta \pi_{3}$ está dada por

$$
\begin{gathered}
\left.\frac{1}{2} \operatorname{Tr}\left(\mathcal{D}_{0}^{-1} \delta \mathcal{D}\right)^{2}\right|_{\left(\delta \pi_{3}\right)^{2}}=-\frac{1}{2} \int \mathcal{G}\left(x^{\prime}-x^{\prime \prime}\right) \mathcal{G}\left(x^{\prime \prime \prime}-x\right) \operatorname{tr}_{c f D}\left[\mathcal{D}_{0}^{-1}\left(x, x^{\prime}\right) \gamma_{5} \exp \left[\Phi\left(x^{\prime}, x^{\prime \prime}\right)\right] \times\right. \\
\left.\mathcal{D}_{0}^{-1}\left(x^{\prime \prime}, x^{\prime \prime \prime}\right) \gamma_{5} \exp \left[\Phi\left(x^{\prime \prime \prime}, x\right)\right]\right] \delta \pi_{3}\left(\frac{x^{\prime}+x^{\prime \prime}}{2}\right) \delta \pi_{3}\left(\frac{x^{\prime \prime \prime}+x}{2}\right)
\end{gathered}
$$

donde la integral se extiende sobre los espacios coordenados $x, x^{\prime}, x^{\prime \prime}$ y $x^{\prime \prime \prime}$, y la traza actúa sobre los espacios de color, sabor y Dirac. 
Realizando las correspondientes transformadas de Fourier, podemos escribir la acción bosonizada en el espacio de momentos como

$$
\begin{aligned}
\left.S_{\mathrm{bos}}\right|_{\left(\delta \pi_{3}\right)^{2}} & =\left.\frac{1}{2} \operatorname{Tr}\left(\mathcal{D}_{0}^{-1} \delta \mathcal{D}\right)^{2}\right|_{\left(\delta \pi_{3}\right)^{2}}+\frac{1}{2 G} \int \frac{d^{4} t}{(2 \pi)^{4}} \delta \pi_{3}(t) \delta \pi_{3}(-t) \\
& =\frac{1}{2} \int \frac{d^{4} t}{(2 \pi)^{4}}\left[F\left(t_{\perp}^{2}, t_{\|}^{2}\right)+\frac{1}{G}\right] \delta \pi_{3}(t) \delta \pi_{3}(-t)
\end{aligned}
$$

$y$, eligiendo el marco de referencia en el que el $\pi^{0}$ se encuentra en reposo, su masa se obtiene como solución de la ecuación

$$
F\left(0,-m_{\pi^{0}}^{2}\right)+\frac{1}{G}=0 .
$$

De este modo, debemos calcular dentro de nuestro modelo la función $F\left(t_{\perp}^{2}, t_{\|}^{2}\right)$ en el límite $t_{\perp}^{2}=0$. A partir de la ecuación (5.33) se obtiene

$$
\begin{aligned}
F\left(0, t_{\|}^{2}\right)= & 16 \pi^{2} N_{C} \sum_{f=u, d} \frac{1}{\left(q_{f} B\right)^{2}} \int_{q_{\perp} p_{\perp} p_{\perp}^{\prime} q_{\|}} g\left(q_{\perp}^{2}+q_{\|}^{2}\right) g\left[\left(p_{\perp}^{\prime}+p_{\perp}-q_{\perp}\right)^{2}+q_{\|}^{2}\right] \times \\
& \exp \left[\frac{i 2 \phi\left(q_{\perp}, p_{\perp}, p_{\perp}^{\prime}\right)}{q_{f} B}\right] \operatorname{tr}_{D}\left[\tilde{S}_{f}\left(p_{\perp}, q_{\|}^{+}\right) i \gamma_{5} \tilde{S}_{f}\left(p_{\perp}^{\prime}, q_{\|}^{-}\right) i \gamma_{5}\right]
\end{aligned}
$$

donde hemos definido $q_{\|}^{ \pm}=q_{\|} \pm t_{\|} / 2$, y la función $\phi$ en la exponencial (siempre considerando el gauge de Landau) está dada por

$$
\phi\left(q_{\perp}, p_{\perp}, p_{\perp}^{\prime}\right)=p_{2} p_{1}^{\prime}+q_{1}\left(p_{2}^{\prime}-p_{2}\right)-p_{1} p_{2}^{\prime}-q_{2}\left(p_{1}^{\prime}-p_{1}\right) .
$$

Para las integrales sobre los vectores de dos dimensiones hemos utilizado la notación

$$
\int_{p q \ldots} \equiv \int \frac{d^{2} p}{(2 \pi)^{2}} \frac{d^{2} q}{(2 \pi)^{2}} \cdots
$$

La evaluación de la traza en (5.36) conduce a

$$
\begin{aligned}
& \operatorname{tr}_{D}\left[\tilde{S}_{f}\left(p_{\perp}, q_{\|}^{+}\right) i \gamma_{5} \tilde{S}_{f}\left(p_{\perp}^{\prime}, q_{\|}^{-}\right) i \gamma_{5}\right]=-8 e^{-\left(p_{\perp}^{2}+p_{\perp}^{\prime 2}\right) / B_{f}} \sum_{k, k^{\prime}=0}^{\infty}(-1)^{k+k^{\prime}} \times \\
& {\left[\sum_{\lambda= \pm} F_{k k^{\prime}, q_{\|}^{+} q_{\|}^{-}}^{\lambda,(A B)} L_{k_{\lambda}}\left(\frac{2 p_{\perp}^{2}}{B_{f}}\right) L_{k_{\lambda}^{\prime}}\left(\frac{2 p_{\perp}^{\prime 2}}{B_{f}}\right)+8 F_{k k^{\prime}, q_{\|}^{+} q_{\|}^{-}}^{+,(C D)}\left(p \cdot p^{\prime}\right) L_{k-1}^{1}\left(\frac{2 p_{\perp}^{2}}{B_{f}}\right) L_{k^{\prime}-1}^{1}\left(\frac{2 p_{\perp}^{\prime 2}}{B_{f}}\right)\right],}
\end{aligned}
$$

con

$$
F_{k k^{\prime}, q_{\|}^{+} q_{\|}^{-}}^{\lambda, f(X Y)} \hat{X}_{k, q_{\|}^{+}}^{\lambda, f} \hat{X}_{k^{\prime}, q_{\|}^{-}}^{\lambda, f}+\left(q_{\|}^{+} \cdot q_{\|}^{-}\right) \hat{Y}_{k, q_{\|}^{+}}^{\lambda, f} \hat{Y}_{k^{\prime}, q_{\|}^{-}}^{\lambda, f},
$$

donde por simplicidad hemos introducido la notación $B_{f}=\left|q_{f} B\right|$, y $X, Y=A, B, C, D$ representan las funciones (5.23-5.26).

66 
Para realizar las integrales sobre $p_{\perp}, p_{\perp}^{\prime} \mathrm{y} q_{\perp}$, que involucran polinomios de Laguerre, es conveniente introducir una transformada de Laguerre-Fourier de los factores de forma no locales, calculando la inversa de la ecuación (5.12):

$$
g\left(p_{\perp}^{2}+p_{\|}^{2}\right)=2 e^{-p_{\perp}^{2} / B_{f}} \sum_{k=0}^{\infty}(-1)^{k_{\lambda}} g_{k, p_{\|}}^{\lambda, f} L_{k_{\lambda}}\left(\frac{2 p_{\perp}^{2}}{B_{f}}\right)
$$

tanto para $\lambda=+$ como $\lambda=-$. Usando esta relación para transformar las funciones $g\left(q_{\perp}^{2}+q_{\|}^{2}\right)$ y $g\left[\left(p_{\perp}^{\prime}+p_{\perp}-q_{\perp}\right)^{2}+q_{\|}^{2}\right]$ en (5.36), se puede mostrar que las integrales sobre las componentes perpendiculares del momento se pueden realizar analíticamente. El cálculo correspondiente, detallado en el Apéndice F, conduce a la expresión [105]

$$
F\left(0, t_{\|}^{2}\right)=-\frac{N_{C}}{\pi} \sum_{f=u, d} B_{f} \sum_{k=0}^{\infty} \int \frac{d^{2} q_{\|}}{(2 \pi)^{2}}\left[\sum_{\lambda= \pm} g_{k, q_{\|}}^{\lambda, f^{2}} F_{k k, q_{\|}^{+} q_{\|}^{-}}^{\lambda, f(A B)}+4 k B_{f} g_{k, q_{\|}}^{+, f} g_{k, q_{\|}^{-, f}}^{-,+, f(C D)} F_{k k, q_{\|}^{+} q_{\|}^{-}}^{+}\right]
$$

En el caso del mesón $\sigma$, la masa se puede determinar a partir de una relación similar a (5.35). La función correspondiente $G\left(0, t_{\|}^{2}\right)$ se obtiene siguiendo los mismos pasos llevados a cabo para el caso del $\pi^{0}$. La diferencia esencial es que se deben quitar los factores $i \gamma_{5}$ en la traza de la ecuación (5.36). Al calcular esta traza se obtiene un resultado análogo a (5.39), donde las nuevas funciones $G_{k k^{\prime}, q_{\|}^{+} q_{\|}^{-}}^{\lambda, f(X Y)}$ están dadas por

$$
\begin{aligned}
G_{k k^{\prime}, q_{\|}^{+} q_{\|}^{-}}^{\lambda, f(A B)} & =-\hat{A}_{k, q_{\|}^{+}}^{\lambda, f} \hat{A}_{k^{\prime}, q_{\|}^{-}}^{\lambda, f}+\left(q_{\|}^{+} \cdot q_{\|}^{-}\right) \hat{B}_{k, q_{\|}^{+}}^{\lambda, f} \hat{B}_{k^{\prime}, q_{\|}^{-}}^{\lambda, f}, \\
G_{k k^{\prime}, q_{\|}^{+} q_{\|}^{-}}^{\lambda, f(C D)} & =\hat{C}_{k, q_{\|}^{+}}^{\lambda, f} \hat{C}_{k^{\prime}, q_{\|}^{\lambda}}^{\lambda, f}-\left(q_{\|}^{+} \cdot q_{\|}^{-}\right) \hat{D}_{k, q_{\|}^{+}}^{\lambda, f} \hat{D}_{k^{\prime}, q_{\|}^{-}}^{\lambda, f}
\end{aligned}
$$

La expresión final para $G\left(0, t_{\|}^{2}\right)$ tiene la misma forma que (5.42), únicamente reemplazando $F_{k k^{\prime}, q_{\|}^{+} q_{\|}^{-}}^{ \pm, f(X Y)} \rightarrow G_{k k^{\prime}, q_{\|}^{+} q_{\|}^{-}}^{ \pm, f(X Y)}$

\section{Constante de decaimiento débil del pion}

La constante de decaimiento débil del pion $f_{\pi^{0}}$ se define a través de la relación (3.46), donde el campo normalizado asociado al estado $\pi^{0}$ en el espacio de momentos es $\tilde{\pi}_{3}(t)=$ $Z_{\pi^{0}}^{-1 / 2} \pi_{3}(t)$. El factor de renormalización de función de onda $Z_{\pi^{0}}^{1 / 2}$ está dado por el residuo del polo del propagador del pion en $t^{2}=-m_{\pi^{0}}^{2}$, que en presencia del campo magnético viene dado por

$$
Z_{\pi^{0}}^{-1}=\left.\frac{d F\left(0, t_{\|}^{2}\right)}{d t_{\|}^{2}}\right|_{t_{\|}^{2}=-m_{\pi^{0}}^{2}}
$$

donde $F\left(0, t_{\|}^{2}\right)$ es la función en la ecuación (5.42). Para el cálculo de $f_{\pi^{0}}$ debemos introducir, a través de la derivada covariante y el transporte paralelo, los acoplamientos con los 
campos de gauge auxiliares, además del acoplamiento con el campo magnético externo. Las expresiones para la derivada covariante y el transporte paralelo resultan

$$
\begin{aligned}
D_{\mu} & =\partial_{\mu}-i \hat{Q} \mathcal{A}_{\mu}(x)-\frac{i}{2} \gamma_{5} \tau_{3} \mathcal{W}_{3 \mu}(x) \\
\mathcal{W}(r, s) & =\mathrm{P} \exp \left\{-i \int_{r}^{s} d \ell_{\mu}\left[\hat{Q} \mathcal{A}_{\mu}(\ell)+\frac{1}{2} \gamma_{5} \tau_{3} \mathcal{W}_{3 \mu}(\ell)\right]\right\} .
\end{aligned}
$$

Necesitamos entonces desarrollar la acción bosonizada a primer orden en $\mathcal{W}_{3}$ y $\delta \pi_{3}$. Escribiendo

$$
\left.S_{\mathrm{bos}}\right|_{W_{3} \delta \pi_{3}}=\int \frac{d^{4} t}{(2 \pi)^{4}} F_{\mu}(t) W_{3 \mu}(t) \delta \pi_{3}(-t)
$$

se encuentra

$$
f_{\pi^{0}}=\left.i Z_{\pi^{0}}^{1 / 2} \frac{t_{\mu} F_{\mu}(t)}{t^{2}}\right|_{t_{\perp}^{2}=0, t_{\|}^{2}=-m_{\pi^{0}}^{2}} .
$$

Para calcular la función $F_{\mu}(t)$ consideraremos nuevamente el desarrollo en (5.32), separando en $\delta \mathcal{D}$ términos con diferentes potencias de $\delta \pi_{3}$ y $W_{3}$ :

$$
\delta \mathcal{D}=\delta \mathcal{D}_{W}+\delta \mathcal{D}_{\pi}+\delta \mathcal{D}_{W \pi}+\ldots
$$

Se tiene así

$$
\left.S_{\mathrm{bos}}\right|_{W_{3} \delta \pi_{3}}=-\operatorname{Tr}\left(\mathcal{D}_{0}^{-1} \delta \mathcal{D}_{W \pi}\right)+\operatorname{Tr}\left(\mathcal{D}_{0}^{-1} \delta \mathcal{D}_{W} \mathcal{D}_{0}^{-1} \delta \mathcal{D}_{\pi}\right)
$$

Los operadores explícitos en la expresión (5.49) son

$$
\begin{aligned}
\delta \mathcal{D}_{\pi}\left(x, x^{\prime}\right)= & i \gamma_{5} \tau_{3} \exp \left[\Phi\left(x, x^{\prime}\right)\right] \mathcal{G}\left(x-x^{\prime}\right) \delta \pi_{3}(\bar{x}) \\
\delta \mathcal{D}_{W}\left(x, x^{\prime}\right)= & \delta^{(4)}\left(x-x^{\prime}\right) \frac{\tau_{3}}{2} \gamma_{5} \gamma_{\mu} W_{3 \mu}(\bar{x}) \\
& +i \bar{\sigma} \gamma_{5} \frac{\tau_{3}}{2} \exp \left[\Phi\left(x, x^{\prime}\right)\right] \mathcal{G}\left(x-x^{\prime}\right)\left[a_{3}(x, \bar{x})-a_{3}(\bar{x}, x)\right] \\
\delta \mathcal{D}_{W \pi}\left(x, x^{\prime}\right)= & -\frac{1}{2} \exp \left[\Phi\left(x, x^{\prime}\right)\right] \mathcal{G}\left(x-x^{\prime}\right)\left[a_{3}(x, \bar{x})-a_{3}(\bar{x}, x)\right] \delta \pi_{3}(\bar{x})
\end{aligned}
$$

donde hemos introducido las definiciones $\bar{x}=\left(x+x^{\prime}\right) / 2 \mathrm{y}$

$$
a_{3}(x, y)=\int_{x}^{y} d \ell_{\mu} W_{3 \mu}(\ell)
$$

y está implícito un producto directo por una matriz identidad en el espacio de color.

El primer y el segundo término en la ecuación (5.50) se pueden entender desde el punto de vista diagramático como una contribución tipo tadpole y una contribución de dos propagadores, respectivamente. Para la de tipo tadpole, al cabo de cierto trabajo se obtiene

$$
-\operatorname{Tr}\left(\mathcal{D}_{0}^{-1} \delta \mathcal{D}_{W \pi}\right)=\int \frac{d^{4} t}{(2 \pi)^{4}} F_{\mu}^{(\mathrm{I})}(t) W_{3 \mu}(t) \delta \pi_{3}(-t),
$$


donde

$$
\begin{aligned}
F_{\mu}^{(\mathrm{I})}(t)= & i \frac{N_{C}}{2} \sum_{f=u, d} \int \frac{d^{4} p}{(2 \pi)^{4}} \frac{d^{4} q}{(2 \pi)^{4}}\left\{g\left[\left(p-\frac{q}{2}\right)^{2}\right]-g\left[\left(p-\frac{q}{2}+\frac{t}{2}\right)^{2}\right]\right\} \times \\
& \operatorname{tr}_{D}\left[\tilde{S}_{f}\left(p_{\perp}, p_{\|}\right)\right] h_{\mu}(q, t-q),
\end{aligned}
$$

con

$$
h_{\mu}\left(q, q^{\prime}\right)=-i \int d^{4} z e^{i q^{\prime} \cdot z} \int_{0}^{z} d \ell_{\mu} e^{i\left(q+q^{\prime}\right) \cdot \ell}
$$

Como estamos interesados en el producto escalar $t \cdot F^{(I)}(t)$, podemos usar la relación

$$
t_{\mu} h_{\mu}(q, t-q)=(2 \pi)^{4}\left[\delta^{(4)}(t-q)-\delta^{(4)}(q)\right]
$$

que resulta independiente del camino elegido para la integral en (5.57). Teniendo en cuenta la expresión para $\tilde{S}_{f}\left(p_{\perp}, p_{\|}\right)$en (5.22) obtenemos

$$
\begin{aligned}
\left.t_{\mu} F_{\mu}^{(\mathrm{I})}(t)\right|_{t_{\perp}=0}= & i 2 N_{C} \sum_{f=u, d} \sum_{k=0}^{\infty} \int_{p_{\perp} p_{\|}}\left[g\left(p^{+^{2}}\right)+g\left(p^{-2}\right)-2 g\left(p^{2}\right)\right] \times \\
& \exp \left(-\frac{p_{\perp}^{2}}{B_{f}}\right) \sum_{\lambda= \pm}(-1)^{k_{\lambda}} \hat{A}_{k, p_{\|}}^{\lambda, f} L_{k_{\lambda}}\left(\frac{2 p_{\perp}^{2}}{B_{f}}\right)
\end{aligned}
$$

donde $p^{ \pm^{2}}=p_{\perp}^{2}+\left(p_{\|} \pm t_{\|} / 2\right)^{2}$. Al igual que para las masas de los mesones, podemos calcular la integral sobre $p_{\perp}$ luego de realizar la transformada de Laguerre-Fourier de los factores de forma no locales, según la ecuación (5.41). Se tiene así

$$
\begin{aligned}
\left.t_{\mu} F_{\mu}^{(\mathrm{I})}(t)\right|_{t_{\perp}=0}= & i 4 N_{C} \sum_{f=u, d} \sum_{k, k^{\prime}=0}^{\infty}(-1)^{k+k^{\prime}} \int_{p_{\perp} p_{\|}} \exp \left(-\frac{2 p_{\perp}^{2}}{B_{f}}\right) \times \\
& \sum_{\lambda= \pm}\left(g_{k^{\prime}, p_{\|}^{+}}^{\lambda, f}+g_{k^{\prime}, p_{\|}^{-}}^{\lambda, f}-2 g_{k^{\prime}, p_{\|}}^{\lambda, f}\right) \hat{A}_{k, p_{\|}}^{\lambda, f} L_{k_{\lambda}^{\prime}}\left(\frac{2 p_{\perp}^{2}}{B_{f}}\right) L_{k_{\lambda}}\left(\frac{2 p_{\perp}^{2}}{B_{f}}\right) \\
= & i \frac{N_{C}}{2 \pi} \sum_{f=u, d} B_{f} \sum_{k=0}^{\infty} \int_{p_{\|}} \sum_{\lambda= \pm}\left(g_{k, p_{\|}^{+}}^{\lambda, f}+g_{k, p_{\|}^{-}}^{\lambda, f}-2 g_{k, p_{\|}}^{\lambda, f}\right) \hat{A}_{k, p_{\|}}^{\lambda, f},
\end{aligned}
$$

donde hemos utilizado la propiedad de ortogonalidad de los polinomios de Laguerre.

Para analizar la contribución de dos propagadores escribimos

$$
\operatorname{Tr}\left(\mathcal{D}_{0}^{-1} \delta \mathcal{D}_{W} \mathcal{D}_{0}^{-1} \delta \mathcal{D}_{\pi}\right)=\int \frac{d^{4} t}{(2 \pi)^{4}}\left[F_{\mu}^{(\mathrm{II})}(t)+F_{\mu}^{(\mathrm{III})}(t)\right] W_{3 \mu}(t) \delta \pi_{3}(-t)
$$

donde $F_{\mu}^{(\mathrm{II})}(t)$ y $F_{\mu}^{(\mathrm{III})}(t)$ corresponden a las contribuciones que surgen del primer y segundo término de $\delta \mathcal{D}_{W}$ en (5.52), respectivamente. Para el primer término se obtiene

$$
\begin{aligned}
F_{\mu}^{(\mathrm{II})}(t)= & i 8 \pi^{2} N_{C} \sum_{f=u, d} \frac{1}{B_{f}^{2}} \int_{q_{\|} q_{\perp} p_{\perp} p_{\perp}^{\prime}} g\left(q^{2}\right) \exp \left[\frac{i 2 \varphi\left(q_{\perp}, p_{\perp}, p_{\perp}^{\prime}, t_{\perp}\right)}{B_{f}}\right] \times \\
& \operatorname{tr}_{D}\left[\tilde{S}_{f}\left(p_{\perp}, q_{\|}^{+}\right) \gamma_{5} \gamma_{\mu} \tilde{S}_{f}\left(p_{\perp}^{\prime}, q_{\|}^{-}\right) \gamma_{5}\right],
\end{aligned}
$$


donde $q_{\|}^{ \pm}=q_{\|} \pm t_{\|} / 2$, y la función $\varphi$ en la exponencial está dada por

$$
\varphi\left(q_{\perp}, p_{\perp}, p_{\perp}^{\prime}, t_{\perp}\right)=p_{2}\left(q_{1}-\frac{t_{1}}{2}\right)-p_{2}^{\prime}\left(q_{1}+\frac{t_{1}}{2}\right)-q_{1} t_{2}-p_{2} p_{1}^{\prime}-(1 \leftrightarrow 2)
$$

Como estamos interesados en el producto $t_{\mu} F_{\mu}^{(\mathrm{II})}(t)$ para $t_{\perp}=0$, calculamos la traza

$$
\begin{aligned}
& \operatorname{tr}_{D}\left[\tilde{S}_{f}\left(p_{\perp}, q_{\|}^{+}\right) \gamma_{5}\left(t_{\|} \cdot \gamma_{\|}\right) \tilde{S}_{f}\left(p_{\perp}^{\prime}, q_{\|}^{-}\right) \gamma_{5}\right]=8 \exp \left[\frac{p_{\perp}^{2}+p_{\perp}^{\prime 2}}{B_{f}}\right] \sum_{k, k^{\prime}=0}^{\infty}(-1)^{k+k^{\prime}} \times \\
& \left\{\sum_{\lambda= \pm}\left[\left(t_{\|} \cdot q_{\|}^{-}\right) \hat{A}_{k, q_{\|}^{+}}^{\lambda, f} \hat{B}_{k^{\prime}, q_{\|}^{-}}^{\lambda, f}-\left(t_{\|} \cdot q_{\|}^{+}\right) \hat{A}_{k^{\prime}, q_{\|}^{-}}^{\lambda, f} \hat{B}_{k, q_{\|}^{+}}^{\lambda, f}\right] L_{k_{\lambda}}\left(\frac{2 p_{\perp}^{2}}{B_{f}}\right) L_{k_{\lambda}^{\prime}}\left(\frac{2 p_{\perp}^{\prime 2}}{B_{f}}\right)\right. \\
& +8 i\left(p_{1} p_{2}^{\prime}-p_{2} p_{1}^{\prime}\right)\left[\left(t_{\|} \cdot q_{\|}^{-}\right) \hat{C}_{k, q_{\|}^{+}}^{+, f} \hat{D}_{k^{\prime}, q_{\|}^{-}}^{+, f}-\left(t_{\|} \cdot q_{\|}^{+}\right) \hat{C}_{k^{\prime}, q_{\|}^{-}}^{+, f} \hat{D}_{k, q_{\|}^{+}}^{+, f}\right] \times \\
& \left.L_{k-1}^{1}\left(\frac{2 p_{\perp}^{2}}{B_{f}}\right) L_{k-1}^{1}\left(\frac{2 p_{\perp}^{2}}{B_{f}}\right)\right\}
\end{aligned}
$$

Ahora podemos introducir la transformada (5.41) para $g\left(q^{2}\right)$, que permite integrar sobre los momentos transversos y escribir el resultado en términos de las transformadas de Laguerre-Fourier de los factores de forma. Este cálculo, descrito en el Apéndice G, conduce a

$$
\begin{aligned}
\left.t_{\mu} F_{\mu}^{(\mathrm{II})}(t)\right|_{t_{\perp}=0}= & -i \frac{N_{C}}{\pi} \sum_{f=u, d} B_{f} \sum_{k=0}^{\infty} \int_{q_{\|}}\left(t_{\|} \cdot q_{\|}^{+}\right) \times \\
& {\left[\sum_{\lambda= \pm} g_{k, q_{\|}}^{\lambda, f} \hat{A}_{k, q_{\|}^{-}}^{\lambda, f} \hat{B}_{k, q_{\|}^{+}}^{\lambda, f}+2 k B_{f}\left(g_{k, q_{\|}}^{+, f}-g_{k, q_{\|}}^{-, f}\right) \hat{C}_{k, q_{\|}^{+}}^{+, f} \hat{D}_{k, q_{\|}^{+}}^{+, f}\right] . }
\end{aligned}
$$

Finalmente, para el segundo término en (5.61) encontramos

$$
\begin{aligned}
F_{\mu}^{(\mathrm{III})}(t)= & i 8 \pi^{2} N_{C} \bar{\sigma} \sum_{f=u, d} \frac{1}{B_{f}^{2}} \int \frac{d^{4} r}{(2 \pi)^{4}} h_{\mu}(r, t-r) \int_{q_{\|} q_{\perp} p_{\perp} p_{\perp}^{\prime}} g\left(q^{2}\right) \times \\
& \left\{g\left[\left(p_{\perp}-\frac{r_{\perp}}{2}-\frac{t_{\perp}}{2}\right)^{2}+\left(p_{\|}+p_{\|}^{\prime}-q_{\|}-\frac{r_{\|}}{2}\right)^{2}\right]\right. \\
& \left.-g\left[\left(p_{\perp}-\frac{r_{\perp}}{2}\right)^{2}+\left(p_{\|}+p_{\|}^{\prime}-q_{\|}-\frac{r_{\|}}{2}+\frac{t_{\|}}{2}\right)^{2}\right]\right\} \times \\
& \exp \left[\frac{i 2 \varphi\left(q_{\perp}, p_{\perp}, p_{\perp}^{\prime}, k_{\perp}\right)}{B_{f}}\right] \operatorname{tr}_{D}\left[\tilde{S}_{f}\left(p_{\perp}, q_{\|}^{+}\right) i \gamma_{5} \tilde{S}_{f}\left(p_{\perp}^{\prime}, q_{\|}^{-}\right) i \gamma_{5}\right],
\end{aligned}
$$

donde la función $\varphi\left(q_{\perp}, p_{\perp}, p_{\perp}^{\prime}, k_{\perp}\right)$ está dada en (5.63). Usando la relación en (5.58) obtenemos 


$$
\begin{aligned}
\left.t_{\mu} F_{\mu}^{(\mathrm{III})}(t)\right|_{t_{\perp}=0}= & i 8 \pi^{2} N_{C} \bar{\sigma} \sum_{f=u, d} \frac{1}{B_{f}^{2}} \int_{q_{\|} q_{\perp} p_{\perp} p_{\perp}^{\prime}}\left[g\left(s_{\perp}^{2}+q_{\|}^{+2}\right)+g\left(s_{\perp}^{2}+q_{\|}^{-2}\right)\right. \\
& \left.-2 g\left(s_{\perp}^{2}+q_{\|}^{2}\right)\right] g\left(q^{2}\right) \exp \left[-\frac{i 2 \phi\left(q_{\perp}, p_{\perp}, p_{\perp}^{\prime}\right)}{B_{f}}\right] \times \\
& \operatorname{tr}_{D}\left[\tilde{S}_{f}\left(p_{\perp}, q_{\|}^{+}\right) i \gamma_{5} \tilde{S}_{f}\left(p_{\perp}^{\prime}, q_{\|}^{-}\right) i \gamma_{5}\right]
\end{aligned}
$$

donde $\phi\left(q_{\perp}, p_{\perp}, p_{\perp}^{\prime}\right)$ está dada en la ecuación (5.37), y hemos definido $s_{\perp}=p_{\perp}^{\prime}+p_{\perp}-q_{\perp}$. Comparando con la ecuación (5.36), se puede ver que el cálculo que se debe llevar a cabo es básicamente el realizado en el caso de la masa del $\pi^{0}$, descrito en el Apéndice F. De este modo se encuentra

$$
\begin{aligned}
\left.t_{\mu} F_{\mu}^{(\mathrm{III})}(t)\right|_{t_{\perp}=0}= & -i \frac{N_{C}}{2 \pi} \bar{\sigma} \sum_{f=u, d} B_{f} \sum_{k=0}^{\infty} \int_{q_{\|}}\left[\sum_{\lambda= \pm} g_{k, q_{\|}}^{\lambda, f} \tilde{g}_{k, q_{\|}}^{\lambda, f} F_{k k, q_{\|}^{+} q_{\|}^{-}}^{\lambda, f(A B)}\right. \\
& \left.+2 k B_{f}\left(g_{k, q_{\|}}^{+, f} \tilde{g}_{k, q_{\|}}^{-, f} t_{\|}+g_{k, q_{\|}}^{-, f} \tilde{g}_{k, q_{\|}}^{+, f} t_{\|}\right) F_{k k, q_{\|}^{+} q_{\|}^{-}}^{+, f(C D)}\right]
\end{aligned}
$$

donde hemos definido

$$
\tilde{g}_{k, q_{\|} t_{\|}}^{\lambda, f}=g_{k, q_{\|}^{+}}^{\lambda, f}+g_{k, q_{\|}^{-}}^{\lambda, f}-2 g_{k, q_{\|}}^{\lambda, f} .
$$

Cuando sumamos las contribuciones dadas en las ecuaciones (5.60), (5.66) y (5.68) podemos ver que hay cancelaciones que ayudan a simplificar la expresión final para $\left.t \cdot F(t)\right|_{t_{\perp}=0}$. Luego de algo de álgebra se llega, en efecto, a

$$
\begin{aligned}
\left.t_{\mu} F_{\mu}(t)\right|_{t_{\perp}=0}= & i \frac{N_{C}}{\pi} \sum_{f=u, d} B_{f} \sum_{k=0}^{\infty} \int_{q_{\|}}\left[\sum_{\lambda= \pm} g_{k, q_{\|}}^{\lambda, f}\left(F_{k k, q_{\|}^{+} q_{\|}^{-}}^{\lambda, f(A B)} M_{k, q_{\|}}^{\lambda, f}-\hat{A}_{k, q_{\|}}^{\lambda, f}\right)\right. \\
& \left.+2 k B_{f}\left(g_{k, q_{\|}}^{+, f} M_{k, q_{\|}}^{-, f}+g_{k, q_{\|}}^{-, f} M_{k, q_{\|}}^{+, f}\right) F_{k k, q_{\|}^{+} q_{\|}^{-}}^{+, f(C D)}\right]
\end{aligned}
$$

La expresión para $f_{\pi^{0}}$ se puede simplificar aún más utilizando la ecuación de gap y la relación (5.35) obtenida para la masa del $\pi^{0}$. Teniendo en cuenta que la ecuación del gap puede escribirse como

$$
\frac{\bar{\sigma}}{G}=\frac{N_{C}}{\pi} \sum_{f=u, d} B_{f} \sum_{k=0}^{\infty} \int_{q_{\|}} \sum_{\lambda= \pm} g_{k, q_{\|}}^{\lambda, f} \hat{A}_{k, q_{\|}}^{\lambda, f},
$$

mientras que para la masa del pion tenemos

$$
\frac{1}{G}=-F\left(0,-m_{\pi^{0}}^{2}\right)
$$

con $F\left(0, t_{\|}^{2}\right)$ dado por (5.42), a partir de estas ecuaciones y la relación en (5.13) se puede observar que existen cancelaciones extra para $t_{\|}^{2}=-m_{\pi^{0}}^{2}$ en (5.70). De este modo, llegamos a una expresión final de la forma

$$
m_{\pi^{0}}^{2} f_{\pi^{0}}=m_{c} Z_{\pi^{0}}^{1 / 2} J\left(-m_{\pi^{0}}^{2}\right),
$$


donde la función $J\left(t_{\|}^{2}\right)$ está dada por

$$
J\left(t_{\|}^{2}\right)=\frac{N_{C}}{\pi} \sum_{f=u, d} B_{f} \sum_{m=0}^{\infty} \int_{q_{\|}} \sum_{\lambda= \pm} g_{k, q_{\|}}^{\lambda, f}\left(F_{k k, q_{\|}^{+} q_{\|}^{-}}^{\lambda, f(A B)}+2 k B_{f} F_{k k, q_{\|}^{\lambda} q_{\|}^{-}}^{\lambda, f(C D)}\right),
$$

$\operatorname{con} q_{\|}^{ \pm}=q_{\|} \pm t_{\|}[105]$

\section{Relaciones quirales}

En esta subsección mostramos que las relaciones de Goldberger-Treiman (GT) y GellMann-Oakes-Renner (GOR) permanecen válidas en nuestro modelo en presencia del campo magnético externo. Análisis previos en este sentido se han llevado a cabo en las Refs. [99] y [100] en el contexto de $\chi$ PT y PIH, respectivamente. Siguiendo el análisis en la Ref. [68], es útil definir la función

$$
K\left(t_{\|}^{2}\right)=m_{c} J\left(t_{\|}^{2}\right)-\bar{\sigma} F\left(0, t_{\|}^{2}\right),
$$

donde $J\left(t_{\|}^{2}\right)$ y $F\left(0, t_{\|}^{2}\right)$ están dadas por las ecuaciones (5.74) y (5.42), respectivamente. De la ecuación (5.70), teniendo en cuenta la relación en (5.13) es sencillo mostrar que

$$
-\left.i t_{\mu} F_{\mu}(t)\right|_{t_{\perp}=0}=K\left(t_{\|}^{2}\right)-\frac{N_{C}}{\pi} \sum_{f=u, d} B_{f} \sum_{k=0}^{\infty} \int_{q_{\|}} \sum_{\lambda= \pm} g_{k, q_{\|}}^{\lambda, f} \hat{A}_{k, q_{\|}}^{\lambda, f} .
$$

A partir de la ecuación del gap, podemos observar que el segundo término es constante, e igual a $-\bar{\sigma} / G$. Además, teniendo en cuenta las relaciones

$$
\begin{gathered}
F_{k,, q_{\|} q_{\|}}^{\lambda, f(A B)}+2 k B_{f} F_{k k, q_{\|} q_{\|}}^{\lambda, f(C D)}=\hat{B}_{k, q_{\|}}^{\lambda, f}, \\
\left(M_{k, q_{\|}}^{ \pm, f}-M_{k, q_{\|}}^{\mp, f}\right) F_{k k, q_{\|} q_{\|}}^{\lambda, f(C D)}=\hat{D}_{k, q_{\|}}^{ \pm, f}, \\
\hat{B}_{k, q_{\|}}^{\lambda, f} M_{k, q_{\|}}^{\lambda, f}-2 k B_{f} \hat{D}_{k, q_{\|}}^{\lambda, f}=\hat{A}_{k, q_{\|}}^{\lambda, f},
\end{gathered}
$$

se puede ver que

$$
m_{c} J(0)-\bar{\sigma} F(0,0)=\frac{N_{C}}{\pi} \sum_{f=u, d} B_{f} \sum_{k=0}^{\infty} \int_{q_{\|}} \sum_{\lambda= \pm} g_{k, q_{\|}}^{\lambda, f} \hat{A}_{k, q_{\|}}^{\lambda, f},
$$

por lo tanto podemos escribir

$$
-\left.i t_{\mu} F_{\mu}(t)\right|_{t_{\perp}=0}=K\left(t_{\|}^{2}\right)-K(0)
$$

De este modo, de la ecuación (5.48) obtenemos

$$
f_{\pi^{0}}=-Z_{\pi^{0}}^{1 / 2} \frac{\left[K\left(-m_{\pi^{0}}^{2}\right)-K(0)\right]}{-m_{\pi^{0}}^{2}} .
$$


En el límite quiral se cumple que $m_{c} \rightarrow 0, m_{\pi^{0}}^{2} \rightarrow 0$, por lo tanto la constante de decaimiento del pion en ese límite vendrá dada por

$$
f_{\pi^{0}, 0}=-\left.Z_{\pi^{0}, 0}^{1 / 2} \frac{d K_{0}\left(t_{\|}^{2}\right)}{d t_{\|}^{2}}\right|_{t_{\|}=0}=\left.Z_{\pi^{0}, 0}^{1 / 2} \bar{\sigma}_{0} \frac{d F_{0}\left(0, t_{\|}^{2}\right)}{d t_{\|}^{2}}\right|_{t_{\|}=0}=Z_{\pi^{0}, 0}^{-1 / 2} \bar{\sigma}_{0},
$$

donde hemos tenido en cuenta la relación entre $Z_{\pi^{0}}$ y la derivada de $F\left(0, t_{\|}^{2}\right)$ en la ecuación (5.44). Los subíndices 0 indican que todas las cantidades tienen que ser evaluadas en el límite quiral. Notando que $Z_{\pi^{0}}^{1 / 2}$ resulta ser la constante de acoplamiento efectiva $g_{\pi q \bar{q}}$ entre el campo $\pi_{3}$ y las corrientes pseudoescalares quark-antiquark, llegamos a

$$
f_{\pi^{0}, 0} g_{\pi q \bar{q}, 0}=\bar{\sigma}_{0}
$$

que es la expresión para la relación de Goldberger-Treiman a nivel de quarks.

Finalmente, consideraremos los condensados de quarks, $\langle\bar{u} u\rangle$ y $\langle\bar{d} d\rangle$, dados por la ecuación (5.28). Teniendo en cuenta las relaciones (5.77), se puede observar que en el límite quiral se obtiene

$$
\langle\bar{u} u+\bar{d} d\rangle_{0}=-\bar{\sigma}_{0} J_{0}(0)
$$

(recordar que fuera del límite quiral las integrales en (5.28) son en general divergentes, y necesitan ser regularizadas). Además, podemos realizar un desarrollo quiral a ambos lados de la ecuación (5.73), dejando únicamente el orden más bajo distinto de cero. Esto conduce a

$$
m_{\pi^{0}}^{2} f_{\pi^{0}, 0}=m_{c} Z_{\pi^{0}, 0}^{1 / 2} J_{0}(0) .
$$

A partir de esta expresión y de la ecuación (5.81), obtenemos la relación de Gell-MannOakes-Renner

$$
m_{c}\langle\bar{u} u+\bar{d} d\rangle_{0}=-m_{\pi^{0}}^{2} f_{\pi^{0}, 0}^{2}
$$

\subsection{Parametrizaciones}

A fin de obtener predicciones numéricas para el comportamiento de las cantidades definidas en la sección anterior como funciones de la temperatura y del campo magnético externo, es necesario especificar la forma funcional del factor de forma no local $g\left(p^{2}\right)$. Consideraremos aquí dos formas utilizadas usualmente: la función gaussiana

$$
g\left(p^{2}\right)=\exp \left(-p^{2} / \Lambda^{2}\right)
$$

y la función "5-Lorentziana"

$$
g\left(p^{2}\right)=\frac{1}{1+\left(p^{2} / \Lambda^{2}\right)^{5}}
$$




\begin{tabular}{ccccc}
$\left(-\langle q \bar{q}\rangle_{0}^{\text {reg }}\right)^{1 / 3}$ & Factor de forma & $m_{c}$ & $G \Lambda^{2}$ & $\Lambda$ \\
\hline 220 & $\mathrm{G}$ & 7,4 & 29,06 & 604 \\
\hline & $\mathrm{L} 5$ & 7,4 & 10,34 & 790 \\
\hline 230 & $\mathrm{G}$ & 6,5 & 23,66 & 678 \\
\hline 240 & $\mathrm{~L} 5$ & 6,5 & 9,700 & 857 \\
\hline & $\mathrm{G}$ & 5,8 & 20,65 & 752 \\
\hline 250 & L5 & 5,8 & 9,267 & 926 \\
\hline & $\mathrm{G}$ & 5,1 & 18,78 & 827 \\
\hline & L5 & 5,1 & 8,958 & 996 \\
\hline
\end{tabular}

Tabla 5.1: Parámetros del modelo para los factores de forma gaussiano y 5-Lorentziano con los cuales se calculan algunos valores representativos del condensado quiral. Los valores de los condensados, las masas corrientes y la constante $\Lambda$ se encuentran expresados en $\mathrm{MeV}$, mientras que $G \Lambda^{2}$ es adimensional.

Estos factores de forma deben incluir un parámetro adicional $\Lambda$ con unidades de energía, que actúa como un cut-off efectivo covariante en el espacio de momentos. En el caso particular de la función gaussiana se tiene la ventaja de que la integral en la ecuación (5.12) puede realizarse analíticamente, dando como resultado

$$
M_{\bar{p}, k}^{\lambda, f}=\left(1-\delta_{k_{\lambda},-1}\right) m_{c}+\bar{\sigma} \frac{\left(1-\left|q_{f} B\right| / \Lambda^{2}\right)^{k_{\lambda}}}{\left(1+\left|q_{f} B\right| / \Lambda^{2}\right)^{k_{\lambda}+1}} \exp \left(-\bar{p}^{2} / \Lambda^{2}\right)
$$

Dados los factores de forma no locales, se deben todavía determinar los valores de los parámetros libres $m_{c}, G$ y $\Lambda$. Consideraremos varios conjuntos de parámetros, obtenidos a partir de exigir que el modelo reproduzca los valores empíricos de la masa y la constante de decaimiento del pion, así como un valor fenomenológicamente aceptable para el condensado $\left\langle\bar{q}_{f} q_{f}\right\rangle_{0}^{\text {reg }}$. Tomaremos en particular los valores $\left(-\left\langle\bar{q}_{f} q_{f}\right\rangle_{0}^{\text {reg }}\right)^{1 / 3}=220,230$, 240 y $250 \mathrm{MeV}$. Los parámetros así obtenidos para los factores de forma gaussiano y 5lorentziano se encuentran en la Tabla 5.1. Las expresiones analíticas utilizadas para calcular la masa y la constante de decaimiento débil del pion para este modelo son las que obtuvimos en el Cap. 3, ecuaciones (3.41), (3.52) y (3.53), en el límite $z(q)=1$ [68].

\subsection{Resultados numéricos}

A continuación discutiremos el comportamiento de los condensados quirales normalizados, de las masas de los mesones $\sigma$ y $\pi$ y de la constante de decaimiento del pion 
como función de $e B$ para los factores de forma introducidos previamente, utilizando las parametrizaciones de la Tabla 5.1.

En los paneles superiores de la Figura 5.1 se observan las predicciones del modelo para $\Delta \bar{\Sigma}_{B}$ como función de $e B$, mientras que en los paneles inferiores se muestran los resultados correspondientes para $\Sigma_{B}^{u}-\Sigma_{B}^{d}$. Por comparación se muestran también los resultados de LQCD presentados en la Ref. [84]. Las curvas sólidas (negras), a rayas (rojas) y punteadas (azules) corresponden a $\left(-\left\langle\bar{q}_{f} q_{f}\right\rangle_{0}^{\text {reg }}\right)^{1 / 3}=220,230$ y $240 \mathrm{MeV}$, respectivamente. Se puede ver que las predicciones de nuestro modelo son muy similares para todas las parametrizaciones consideradas, y muestran un buen acuerdo con los resultados de LQCD. En el caso de $\Sigma_{B}^{u}-\Sigma_{B}^{d}$ puede notarse cierta dependencia con la parametrización, obteniéndose para ambos factores de forma un mejor acuerdo con los datos de LQCD en el caso de los parámetros correspondientes al condensado $\left(-\left\langle\bar{q}_{f} q_{f}\right\rangle_{0}^{\text {reg }}\right)^{1 / 3}=230 \mathrm{MeV}$.
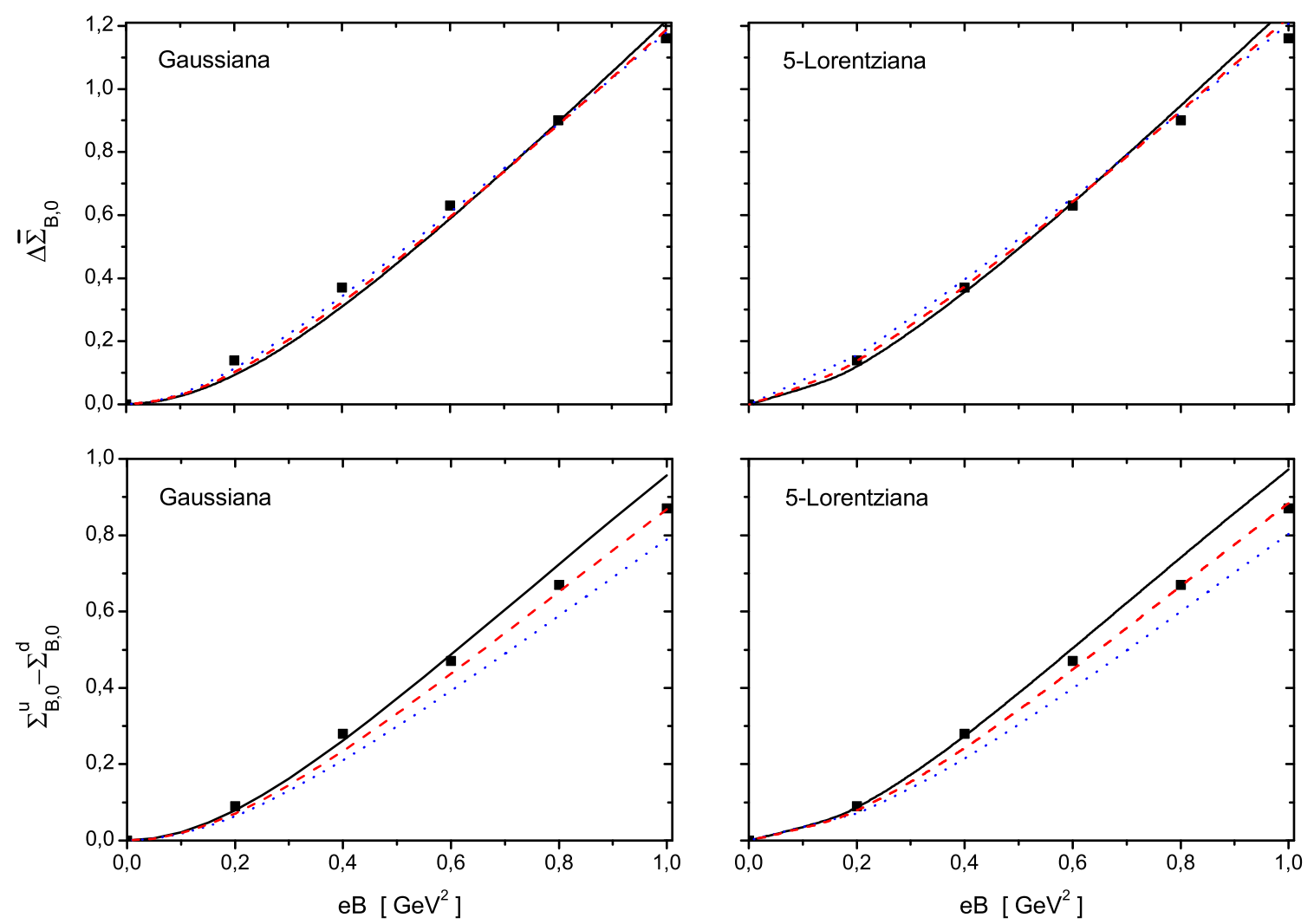

Figura 5.1: Condensados normalizados en función de campo magnético. Panel superior: promedio de sabor; panel inferior: diferencia de sabores. Las curvas sólidas (negras), a rayas (rojas) y punteadas (azules) corresponden a las parametrizaciones que conducen a $\left(-\langle\bar{q} q\rangle_{0}^{\text {reg }}\right)^{1 / 3}=220,230$ y $240 \mathrm{MeV}$, respectivamente. Los símbolos cuadrados indican los resultados de LQCD tomados de la Ref. [84]. 
El aumento de los condensados quirales en función del campo magnético que se puede observar en la Figura 5.1 refleja la llamada catálisis magnética. Debido a que los condensados quirales son los parámetros de orden de la ruptura dinámica de la simetría quiral, este efecto puede interpretarse como una intensificación de dicha ruptura debida a la presencia del campo magnético. El fenómeno puede entenderse como consecuencia de la tendencia del campo magnético a fortalecer la unión de partículas de cargas opuestas en los condensados.

Nuestros resultados para el comportamiento de la masa del pion $m_{\pi^{0}}(B)$ y el cuadrado de la constante de decaimiento del pion $f_{\pi^{0}}^{2}(B)$ en función de $e B$ se pueden observar en las Figuras 5.2 y 5.3 respectivamente. En ambos casos las curvas han sido normalizadas para los valores a $B=0$ siendo $m_{\pi^{0}}(0)=139 \mathrm{MeV}$ y $f_{\pi^{0}}^{2}(0)=(92,4 \mathrm{MeV})^{2}$. La parametrización utilizada fue la correspondiente al condensado $\left(-\left\langle\bar{q}_{f} q_{f}\right\rangle_{0}^{\text {reg }}\right)^{1 / 3}=230 \mathrm{MeV}$ tanto para el factor de forma gaussiano como el 5-lorentziano. No se incluyen resultados para otros valores de $\left(-\left\langle\bar{q}_{f} q_{f}\right\rangle_{0}^{\text {reg }}\right)^{1 / 3}$ dado que las curvas en las Figuras 5.2 y 5.3 se mantienen prácticamente sin variación cuando se utilizan parametrizaciones que conducen a condensados a $B=0$ entre $-(220 \mathrm{MeV})^{3} \mathrm{y}-(250 \mathrm{MeV})^{3}$.

Como se observa en la Figura 5.2, la masa del $\pi^{0}$ decrece a medida que $e B$ aumenta, llegando a un valor cercano al $65 \%$ de $m_{\pi^{0}}(0)$ a $e B \simeq 1,5 \mathrm{GeV}^{2}$, el cual corresponde a un campo magnético de alrededor de $2,5 \times 10^{20} \mathrm{G}$. También se incluye en la figura una banda gris que corresponde a los resultados presentados recientemente por LQCD [93]. A causa de la problemática que se presenta en los cálculos de LQCD para obtener las masas de los mesones livianos, estos resultados fueron calculados a partir de una extrapolación al continuo del espaciado de la red, considerando una masa relativamente alta para el pion, $m_{\pi^{0}}=415 \mathrm{MeV}$. Por comparación también mostramos los resultados obtenidos dentro de nuestro modelo variando el valor del parámetro $m_{c}$ a $56,3 \mathrm{MeV}$, lo cual conduce a este valor de $m_{\pi^{0}}$. En general, se puede ver que nuestras predicciones resultan estar de acuerdo con los cálculos de LQCD. Es importante tener en cuenta que a diferencia de otros modelos, nuestros resultados fueron obtenidos directamente a partir de las parametrizaciones utilizadas en el caso de $B=0$ en trabajos anteriores, sin realizar ajustes para que concuerden con los datos provenientes de LQCD. Con respecto a la constante de decaimiento del pion $f_{\pi^{0}}$, como se observa en la Figura 5.3, encontramos que ésta se comporta como una función creciente de B. Esto es perfectamente consistente con la relación de Gell-Mann-OakesRenner para un valor pequeño de la masa constituyente $m_{\mathcal{c}}$. Teniendo en cuenta el comportamiento de la masa del pion y el resultado en la ecuación (5.85), $f_{\pi^{0}}^{2}(B)$ debe crecer más rápidamente que los condensados, lo cual puede observarse al comparar con las curvas en la Figura 5.1. Por ejemplo, para $e B=1,5 \mathrm{GeV}^{2}$ se obtiene $m_{c}\langle\bar{u} u+\bar{d} d\rangle /\left(m_{\pi^{0}}^{2} f_{\pi^{0}}^{2}\right) \simeq-0,98$ para los factores de forma gaussiano y 5-lorentziano, en acuerdo con la ecuación (5.85). 


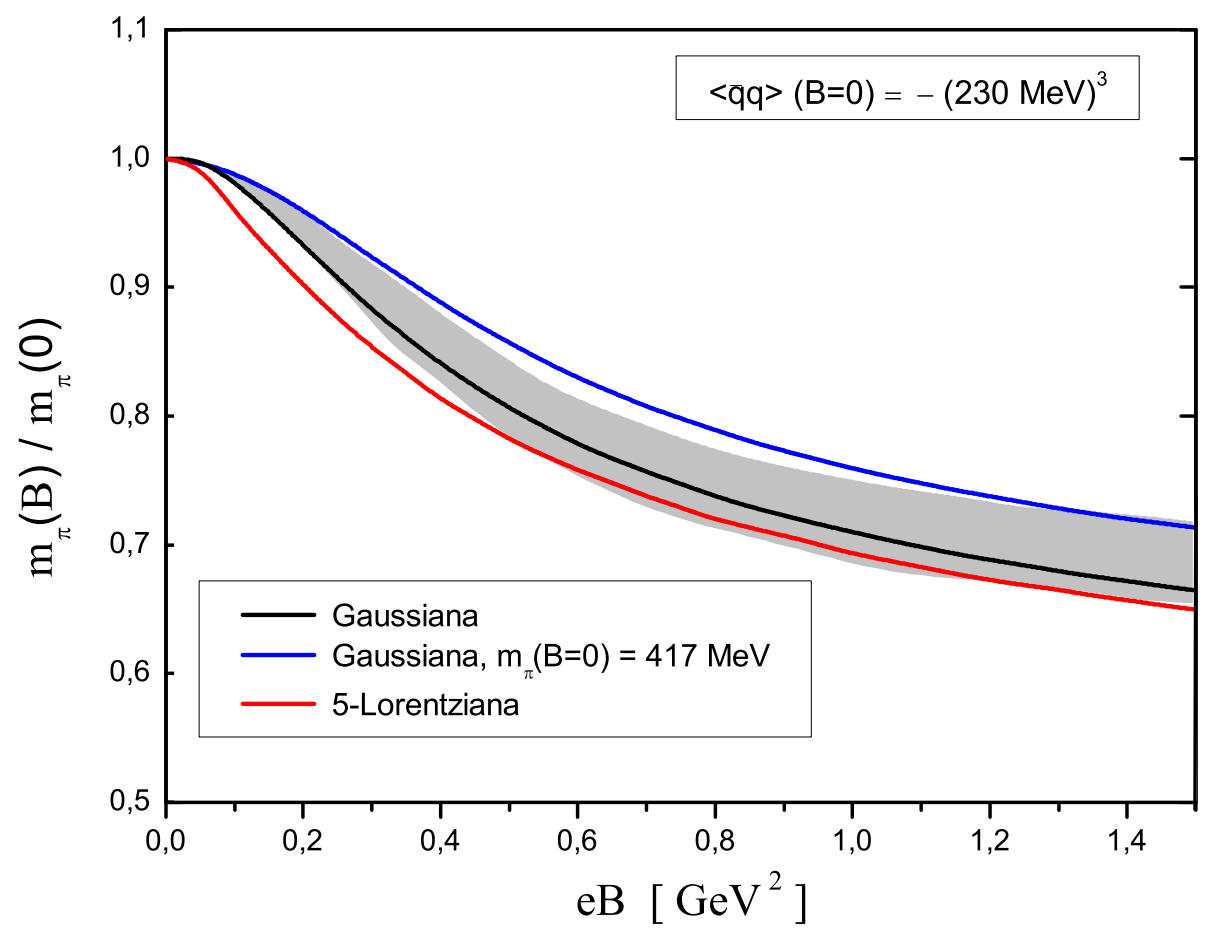

Figura 5.2: Masas del mesón $\pi^{0}$ en función de $e B$. Las curvas negra y roja a las parametrizaciones de $\left(-\langle\bar{q} q\rangle_{0}^{\text {reg }}\right)^{1 / 3}=230 \mathrm{MeV}$, con el factor de forma gaussiano y 5-lorentziano respectivamente. La curva azul resultada de una parametrización en la que $m_{\pi^{0}}=417 \mathrm{MeV}$ con el factor de forma gaussiano, mientras que la banda gris indica los resultados de LQCD tomados de la Ref. [93].

Finalmente, en la Figura 5.4 se presentan los valores para el masa del mesón $\sigma$ normalizada a $m_{\sigma}(0)$. En este caso los resultados resultan ser más dependientes de la parametrización, por lo cual hemos considerado tres conjuntos de parámetros correspondientes a los condensados $\left(-\left\langle\bar{q}_{f} q_{f}\right\rangle_{0}^{\text {reg }}\right)^{1 / 3}=230,240$ y $250 \mathrm{MeV}$ con factor de forma gaussiano. Los valores obtenidos para $m_{\sigma}(0)$ son 771, 683 y $616 \mathrm{MeV}$, respectivamente. A diferencia del caso de $m_{\pi^{0}}$, las curvas para $m_{\sigma}$ presentan un máximo antes de comenzar a decrecer. Para valores menores del condensado a $B=0$, así como en el caso del factor de forma 5-lorentziano, la determinación de la masa del mesón $\sigma$ se vuelve problemática ya que excede el umbral a partir del cual puede tener lugar un decaimiento en dos quarks. Esta situación es similar a la descrita en la Sec. 4.3 cuando se analizó el caso de la masa del mesón axial $f_{1}$. 


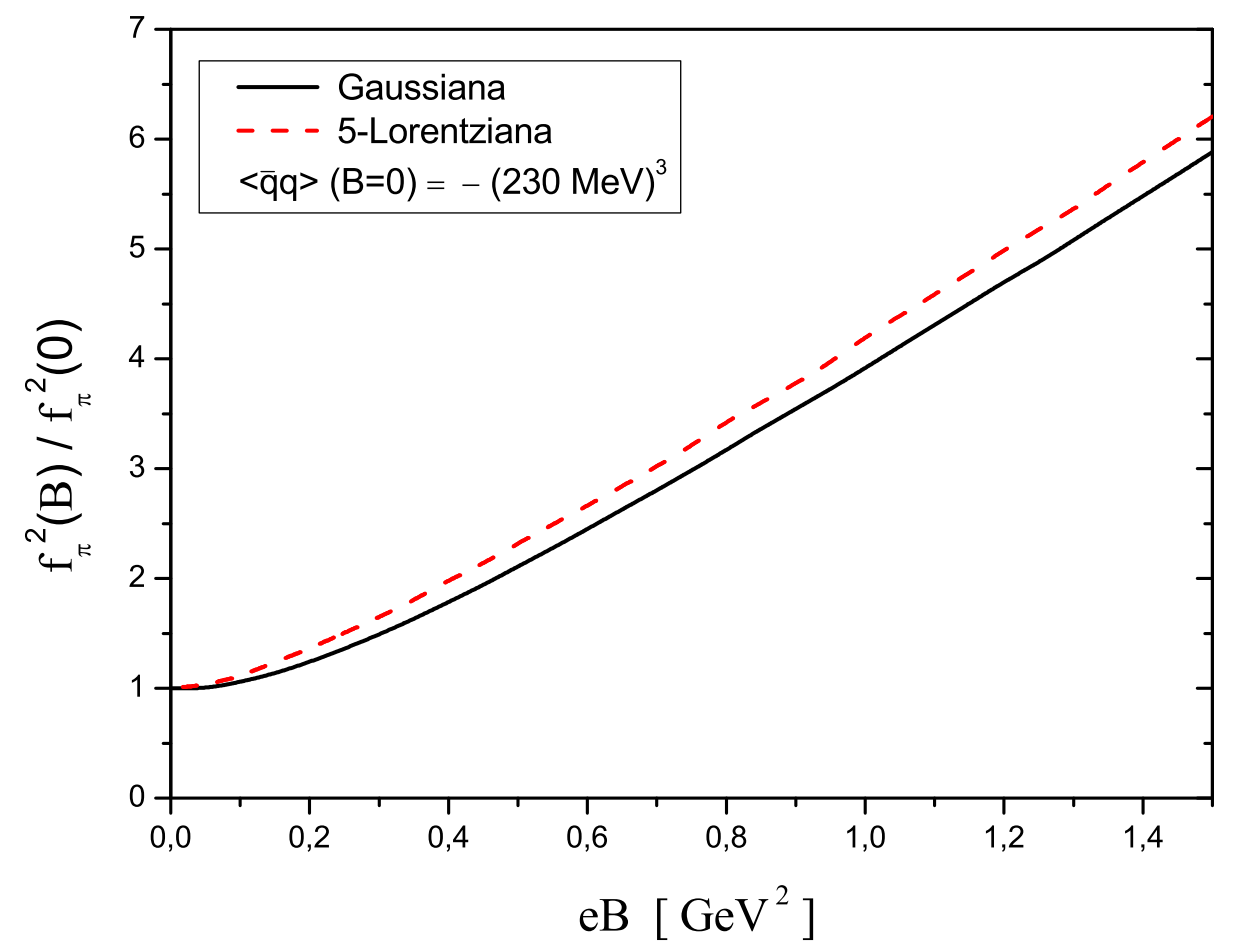

Figura 5.3: Constante de decaimiento del pion al cuadrado normalizada en función del campo magnético externo. Las curvas sólida (negra) y a rayas (roja) corresponden a parametrizaciones con los factores de forma gaussiano y 5-lorentziano, respectivamente. 


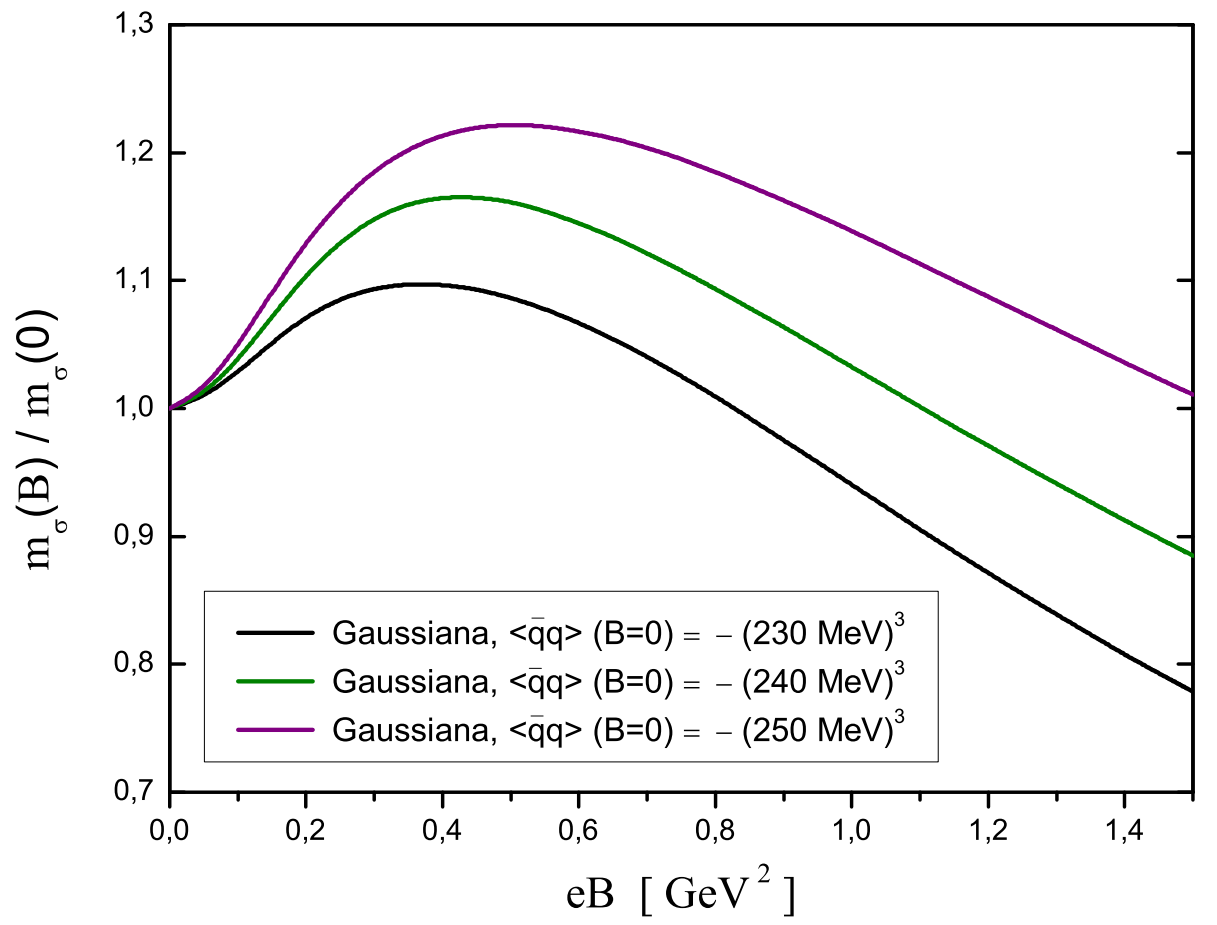

Figura 5.4: Masa del mesón $\sigma$ en función de $e B$ normalizada a partir de su valor correspondiente para $B=0$. Las curvas negras, verde y violeta corresponden a las parametrizaciones de $\left(-\langle\bar{q} q\rangle_{0}^{\text {reg }}\right)^{1 / 3}=230,240$ y $250 \mathrm{MeV}$, respectivamente utilizando el factor de forma gaussiano. 



\section{Capítulo 6}

\section{Modelo NJL no local a temperatura finita en presencia de campo magnético}

En este capítulo extenderemos el modelo NJL no local de dos sabores de quarks al régimen de temperatura $T$ finita, con lo cual podremos estudiar las predicciones del mismo para las transiciones de fases quiral y de deconfinamiento en función de la temperatura. Para ello se utilizará el formalismo de Matsubara para la incorporación de la temperatura y se incluirá un acoplamiento con el loop de Polyakov, que permite una descripción del confinamiento. Detallaremos seguidamente el modo en que puede introducirse en este marco la interacción con un campo magnético externo uniforme.

\subsection{Modelo NJL local a temperatura finita}

\subsubsection{Formalismo de Matsubara o de tiempo imaginario}

Para describir un sistema a temperatura finita en el marco de una teoría cuántica de campos es usual considerar la matriz densidad $\hat{\rho}$, cuyos valores de expectación $\langle\phi|\hat{\rho}| \phi\rangle$ indican la probabilidad de que, para una temperatura $T$ específica, el sistema se encuentre en el estado $|\phi\rangle$. El comportamiento estadístico de un sistema cuántico en equilibrio térmico puede estudiarse a través de su función de partición, definida como

$$
\mathcal{Z}(\beta)=\operatorname{Tr} \hat{\rho}(\beta)
$$

donde la traza es la suma sobre todos los valores de expectación en cualquier base completa, y $\beta$ es la recíproca de la temperatura (se utilizan unidades tales que la constante de Boltzman es $k=1$ ). La función de partición $\mathcal{Z}$ es una herramienta mediante la cual pueden 
obtenerse todas las propiedades termodinámicas del sistema. El promedio estadístico del valor esperado de un observable $\hat{A}$ viene dado por

$$
\langle A\rangle=\mathcal{Z}(\beta)^{-1} \operatorname{Tr}[\hat{\rho}(\beta) \hat{A}]=\frac{\operatorname{Tr}[\hat{\rho}(\beta) \hat{A}]}{\operatorname{Tr} \hat{\rho}(\beta)} .
$$

En general para estudiar la estadística de sistemas con la posibilidad de creación y destrucción de partículas, se debe considerar la matriz densidad para el ensamble gran canónico,

$$
\hat{\rho}=\exp (-\beta \hat{\mathcal{H}}), \quad \text { donde } \quad \hat{\mathcal{H}}=\hat{H}-\mu \hat{N} .
$$

Aquí $\hat{H}$ y $\hat{N}$ son los operadores hamiltoniano y número de partículas respectivamente, y $\mu$ es el potencial químico. En esta Tesis nos concentraremos en particular en el caso $\mu=0$ ( $N$ fijo).

Para el tratamiento de sistemas con temperatura finita consideraremos el formalismo de Matsubara o de tiempo imaginario. La idea básica consiste en que los valores de expectación de operadores en un ensamble térmico se pueden reescribir como valores de expectación en teoría cuántica de campos, donde la configuración evoluciona en un tiempo imaginario o euclídeo $T=i t$. De esta forma, se puede realizar una transformación a un espacio-tiempo con métrica euclídea, donde la traza de la ecuación (6.2) lleva a requerir que los campos bosónicos y fermiónicos sean respectivamente periódicos o anti-periódicos con respecto a la dirección del tiempo euclídeo, con periodicidad $\beta$. En el espacio de momentos, esto conduce al reemplazo de frecuencias continuas por frecuencias discretas. Esto se puede comprender fácilmente dentro del contexto de integrales funcionales de camino. Para ello consideraremos el caso de campo escalar $\phi$. La amplitud de transición entre dos estados en una teoría cuántica de campos viene dada por

$$
\left\langle\phi\left(\vec{x}_{1}, t_{1}\right) \mid \phi\left(\vec{x}_{2}, t_{2}\right)\right\rangle=\left\langle\phi_{1}\left|\exp \left(-i \mathcal{H}\left(t_{1}-t_{2}\right)\right)\right| \phi_{2}\right\rangle=N^{\prime} \int \mathcal{D} \phi e^{i S},
$$

donde $N^{\prime}$ es una constante de normalización y $S$ la acción definida por

$$
S=\int_{t_{2}}^{t_{1}} d t \int d^{3} x \mathcal{L}
$$

con $\mathcal{L}$ representando la densidad lagrangiana apropiada para el sistema. La integral funcional está definida sobre caminos cuyos extremos se mantienen fijos,

$$
\phi\left(\vec{x}_{1}, t_{1}\right)=\phi_{1}, \quad \phi\left(\vec{x}_{2}, t_{2}\right)=\phi_{2} .
$$

Dadas las tres últimas ecuaciones es fácil ver que identificando $t_{1}-t_{2}=-i \beta$, se puede escribir la función de partición para cualquier sistema cuántico en la base de estados $\left|\phi_{a}\right\rangle$ como

$$
\mathcal{Z}(\beta)=\operatorname{Tr} e^{-\beta \mathcal{H}}=\int d \phi_{a}\left\langle\phi_{a}\left|e^{-\beta \mathcal{H}}\right| \phi_{a}\right\rangle=N^{\prime} \int \mathcal{D} \phi e^{-S_{E}}
$$


donde $S_{E}$, la acción euclídea (tiempo imaginario), es

$$
S_{E}=\int_{0}^{\beta} d \tau \int d^{3} x \mathcal{L}_{\mathcal{E}}
$$

mientras que los campos satisfacen condiciones de contorno periódicas

$$
\phi(\vec{x}, \beta)=\phi(\vec{x}, 0)
$$

Cuando pasamos al espacio de momentos, encontramos que la integración sobre un intervalo finito implica que las frecuencias en la transformada de Fourier toman valores discretos.

Las distintas condiciones de contorno determinan los posibles valores para dichas frecuencias, conocidas como frecuencias o modos de Matsubara. A diferencia de los campos bosónicos, los campos fermiónicos satisfacen condiciones de contorno antiperiódicas, por lo tanto las frecuencias de Matsubara vienen dadas por

$$
\omega_{n}= \begin{cases}\frac{2 n \pi}{\beta} & \text { para bosones } \\ \frac{(2 n+1) \pi}{\beta} & \text { para fermiones. }\end{cases}
$$

donde $n$ es un entero.

En conclusión, el formalismo de Matsubara propone la siguiente sustitución en las integrales sobre momentos consideradas en los modelos de los capítulos anteriores:

$$
\begin{aligned}
\int \frac{d^{4} q}{(2 \pi)^{4}} F(q) & =\int \frac{d q_{0}}{2 \pi} \int \frac{d^{3} \vec{q}}{(2 \pi)^{3}} F\left(q_{0}, \vec{q}\right) \\
& \rightarrow T \sum_{n=-\infty}^{\infty} \int \frac{d^{3} \vec{q}}{(2 \pi)^{3}} F\left(\omega_{n}, \vec{q}\right)
\end{aligned}
$$

donde $F(q)$ representa al integrando en cada caso.

\subsubsection{Loop de Polyakov}

El loop de Polyakov fue propuesto por A.M. Polyakov [31] como una aplicación del loop de Wilson al problema de propiedades térmicas de campos de gauge, en particular como un mecanismo que explicara el deconfinamiento de quarks a una cierta temperatura. Para introducirlo es conveniente primero mencionar brevemente la simetría involucrada.

\section{Simetría global Z(N) en SU(N)}

Tal como se indica en el trabajo de $t^{\prime}$ Hooft [106], en una teoría de gauge SU(N) local existe una simetría global $\mathrm{Z}(\mathrm{N})$. Para ver esto partimos de una densidad lagrangiana que 
incluya la interacción de los quarks con los campos gluónicos

$$
\mathcal{L}=\frac{1}{2} \operatorname{Tr} G_{\mu \nu}^{2}+\bar{\psi} i \gamma^{\mu} D_{\mu} \psi,
$$

donde

$$
D_{\mu}=\partial_{\mu}-i g A_{\mu} \quad \text { y } \quad G_{\mu v}=\frac{i}{g}\left[D_{\mu}, D_{\nu}\right] .
$$

El campo $A_{\mu}$ está definido según $A_{\mu}=A_{\mu}^{a} t^{a}$, donde las matrices $t^{a}$ son los generadores del grupo SU(N) normalizados como $\operatorname{Tr}\left(t^{a} t^{b}\right)=\delta^{a b} / 2$. Este lagrangiano es invariante ante transformaciones de gauge $\Omega$, dadas por

$$
D_{\mu} \rightarrow \Omega^{\dagger} D_{\mu} \Omega, \quad \psi \rightarrow \Omega^{\dagger} \psi .
$$

$\mathrm{Al}$ ser un elemento de $\mathrm{SU}(\mathrm{N}), \Omega$ satisface que

$$
\Omega^{\dagger} \Omega=\mathbb{1}, \quad \operatorname{det} \Omega=1 .
$$

Siendo una transformación de gauge local, $\Omega$ es en general función de las coordenadas espacio-temporales.

Consideremos una transformación de gauge global dada por una fase constante por la matriz unidad:

$$
\Omega_{c}=e^{-i \varphi} \mathbb{1} .
$$

Para que esta transformación sea un elemento de SU(N), el determinante debe ser igual a uno, lo cual requiere que

$$
\varphi=\frac{2 \pi j}{N}, \quad j=0,1, \ldots(N-1) .
$$

Esto determina un grupo cíclico, cuyos elementos son generados por las potencias $j$ de un único elemento, definiendo así una simetría global $\mathrm{Z}(\mathrm{N})$. Se dice que estos elementos conforman el centro del grupo SU(N).

\section{$\mathrm{Z}(\mathrm{N})$ a temperatura finita}

Al ser un subgrupo de las transformaciones de gauge, las rotaciones del grupo $\mathrm{Z}(\mathrm{N})$ son siempre una simetría del Lagrangiano, con quarks o sin ellos. Sin embargo, en presencia de quarks dinámicos, las rotaciones de $\mathrm{Z}(\mathrm{N})$ no son una simetría de la teoría ya que violan las condiciones de borde requeridas.

En efecto, trabajando en un espacio-tiempo euclídeo a una temperatura $T$, la coordenada de tiempo imaginario $\tau$ es de extensión finita, $\tau: 0 \rightarrow \beta=1 / T$. Las condiciones de borde que deben satisfacer los campos están dadas por la estadística propia de cada uno de ellos. Esto es, los gluones deben ser periódicos en $\tau$, mientras que los quarks deben ser anti-periódicos:

$$
A_{\mu}(\vec{x}, \beta)=+A_{\mu}(\vec{x}, 0), \quad \psi(\vec{x}, \beta)=-\psi(\vec{x}, 0) .
$$


Cualquier transformación de gauge que sea periódica en $\tau$ respeta estas condiciones de borde. Sin embargo, $t^{\prime}$ Hooft encontró que es posible considerar transformaciones de gauge más generales, las cuales son periódicas a menos de $\Omega_{c}$ :

$$
\Omega(\vec{x}, \beta)=\Omega_{c}, \quad \Omega(\vec{x}, 0)=1 .
$$

Los campos de color adjuntos son invariantes ante esta transformación, mientras que los que están en la representación fundamental no lo son:

$$
\begin{aligned}
& A^{\Omega}(\vec{x}, \beta)=\Omega_{c}^{\dagger} A_{\mu}(\vec{x}, \beta) \Omega_{c}=A_{\mu}(\vec{x}, \beta)=+A_{\mu}(\vec{x}, 0), \\
& \psi^{\Omega}(\vec{x}, \beta)=\Omega_{c}^{\dagger} \psi(\vec{x}, \beta)=e^{i \varphi} \psi(\vec{x}, \beta) \neq-\psi(\vec{x}, 0) .
\end{aligned}
$$

Aquí se ha utilizado el hecho de que $\Omega_{c}$, al ser una fase constante por la matriz unitaria, conmuta con cualquier matriz de SU(N). En consecuencia, las teorías de gauge SU(N) "puras" (sin fermiones) tienen una simetría global $\mathrm{Z}(\mathrm{N})$, la cual deja de ser exacta al incluir quarks dinámicos.

En la teoría de gluones pura, un parámetro de orden para la simetría $\mathrm{Z}(\mathrm{N})$ se construye utilizando la línea de Wilson térmica:

$$
L(\vec{x})=\mathcal{P} \exp \left(i g \int_{0}^{\beta} A_{0}(\vec{x}, \tau) d \tau\right),
$$

donde $g$ es la constante de acoplamiento de gauge, $A_{0}$ es el vector potencial en la dirección temporal, y el símbolo $\mathcal{P}$ denota ordenamiento de camino. Con esta definición, la línea de Wilson térmica transforma como un campo adjunto ante transformaciones de gauge SU(N) locales:

$$
L(\vec{x}) \rightarrow \Omega^{\dagger}(\vec{x}, \beta) L(\vec{x}) \Omega(\vec{x}, 0) .
$$

El loop de Polyakov [31] se define como la traza de la línea de Wilson térmica, y es, por lo tanto, invariante de gauge:

$$
\phi(\vec{x})=\frac{1}{N} \operatorname{Tr} L=N^{-1} \operatorname{Tr} \mathcal{P} \exp \left(i g \int_{0}^{\beta} A_{0}(\vec{x}, \tau) d \tau\right) .
$$

Ante transformaciones globales $\mathrm{Z}(\mathrm{N})$, el loop de Polyakov $\phi$ transforma como un campo con carga uno:

$$
\phi \rightarrow e^{i \varphi} \phi
$$

A muy alta temperatura $g \approx 0$, por lo que se esperaría que $\langle\phi\rangle \sim 1$. Sin embargo, el vacío permitido exhibe una degeneración de $N$ hojas. Esto es,

$$
\langle\phi\rangle=\exp \left(\frac{i 2 \pi j}{N}\right) \phi_{0}, \quad j=0,1, \ldots,(N-1),
$$


donde $\phi_{0}$ es una función real, que además cumple que $\phi_{0} \rightarrow 1$ cuando $T \rightarrow \infty$. Cualquier valor de $j$ es equivalente, por lo que cualquier elección conduce a la ruptura espontánea de la simetría global $\mathrm{Z}(\mathrm{N})$.

A temperatura cero, el confinamiento implica que $\phi_{0}$ se cancela [32]. Por lo tanto, debe existir cierto valor de temperatura $T_{\mathcal{c}}$ a partir del cual $\phi_{0}$ deja de ser nulo y se produce el deconfinamiento. Esto es

$$
\left\{\begin{array}{lll}
\phi_{0}=0 & \text { si } & T<T_{c} \\
\phi_{0}>0 & \text { si } & T>T_{c} .
\end{array}\right.
$$

Como es habitual, si $\phi_{0}$ se vuelve distinto de cero en forma continua alrededor de $T_{\mathcal{c}}$, la transición es de segundo orden, mientras que si se produce un salto en $T=T_{\mathcal{c}}$ es de primer orden.

Por otro lado, en presencia de quarks dinámicos la simetría $\mathrm{Z}(\mathrm{N})$ está explícitamente rota. En este caso entonces, el loop de Polyakov deja de ser un parámetro de orden riguroso, pero sirve aún como indicador de un crossover hacia el deconfinamiento.

Recientemente se ha propuesto una extensión del modelo NJL incluyendo al loop de Polyakov [34]. La idea principal es introducir a $\phi$ como un campo efectivo que se acople con los quarks siguiendo las reglas dictadas por las simetrías y patrones de ruptura de simetría de QCD, unificando así los aspectos de confinamiento y ruptura de simetría quiral. Se suele referir a este esquema como modelo PNJL (NJL extendido con loop de Polyakov).

\subsubsection{Modelo Polyakov-Nambu-Jona Lasinio (PNJL)}

La acción a temperatura $T$ finita se puede obtener utilizando el formalismo de Matsubara de tiempo imaginario, tal como se explicó en la Sec. 6.1.1, y para estudiar el comportamiento de la materia fuertemente interactuante de modo de incluir una descripción del deconfinamiento se propone incorporar el acoplamiento al loop de Polyakov (PL). Para ello se puede hacer uso de algunas características conocidas de su comportamiento en función de la temperatura, introduciendo un modelo efectivo para la teoría de gauge pura, que luego se acople al modelo de NJL. Dentro de las propiedades generales que debe tener el potencial efectivo de Polyakov, éste debe satisfacer la simetría global Z(3) al igual que las simetrías del lagrangiano puro SU(3) de Yang-Mills [66, 107, 108, 109, 110, 111].

Como hemos visto puede identificarse al valor de expectación de la traza del loop de Polyakov $\Phi=\langle\phi\rangle$ como un parámetro de orden, siendo $\Phi=0$ el valor correspondiente a la fase en que los quarks y gluones están confinados. Cálculos de LQCD para el valor de la traza del PL como función de la temperatura indican que la transición de deconfinamiento es ausencia de quarks dinámicos ocurre a una temperatura $T_{0}=270 \mathrm{MeV}$, siendo esta transición de primer orden. 
Es posible construir un potencial efectivo $\mathcal{U}(\Phi, T)$ de acuerdo a las estimaciones de LQCD. Éste deberá tener un mínimo en $\Phi=0$ cuando $T<T_{0}$, y en $T=T_{0}$ pasar a tener un máximo local en $\Phi=0$ y un mínimo en un valor $\Phi>0$, dando lugar a la transición de fase. Esto se corresponde con la ruptura de simetría Z(3). El mínimo debe acercarse a $\Phi=1$ cuando la temperatura continúa aumentando por encima de $T_{0}$. A continuación se describirán dos potenciales propuestos en la literatura.

En primer lugar consideraremos un potencial efectivo basado en la forma logarítmica de la medida de Haar de integración asociada con el grupo SU(3) de color [112]. La forma funcional de este potencial es

$$
\frac{\mathcal{U}_{\log }\left(\Phi, \Phi^{*}, T\right)}{T^{4}}=-\frac{1}{2} a(T)\left(\Phi \Phi^{*}\right)+b(T) \log \left[1-6\left(\Phi \Phi^{*}\right)+4\left(\Phi^{3}+\Phi^{* 3}\right)-3\left(\Phi \Phi^{*}\right)^{2}\right],
$$

con los coeficientes $a(T)$ y $b(T)$ parametrizados según

$$
a(T)=a_{0}+a_{1}\left(\frac{T_{0}}{T}\right)+a_{2}\left(\frac{T_{0}}{T}\right)^{2} \quad \text { y } \quad b(T)=b_{3}\left(\frac{T_{0}}{T}\right)^{3} .
$$

Los parámetros del potencial están ajustados de manera tal que se puede reproducir la ecuación de estado para la teoría de gauge pura y el valor asintótico de expectación del loop de Polyakov cuando $T \rightarrow \infty$. Esto conduce a

$$
a_{0}=3,51, a_{1}=-2,47, a_{2}=15,2, a_{3}=-1,75 .
$$

Una forma alternativa, basada en el ansatz de Ginzburg-Landau [34, 113] viene dada por una función polinómica de la forma

$$
\frac{\mathcal{U}_{\text {Poly }}\left(\Phi, \Phi^{*} ; T\right)}{T^{4}}=-\frac{b_{2}(T)}{2} \Phi \Phi^{*}-\frac{b_{3}}{3}\left(\Phi^{3}+\Phi^{* 3}\right)+\frac{b_{4}}{4}\left(\Phi \Phi^{*}\right)^{2},
$$

donde

$$
b_{2}(T)=a_{0}+a_{1}\left(\frac{T_{0}}{T}\right)+a_{2}\left(\frac{T_{0}}{T}\right)^{2}+a_{3}\left(\frac{T_{0}}{T}\right)^{3} .
$$

Ajustando una vez más el valor de las constantes a resultados de la teoría de gauge pura en LQCD, se obtiene

$$
\begin{gathered}
a_{0}=6,75, a_{1}=-1,95, a_{2}=2,625, \\
a_{3}=-7,44, \quad b_{3}=0,75, \quad b_{4}=7,5 .
\end{gathered}
$$

En relación al parámetro $T_{0}$, que fija la escala para el potencial del PL, se ha argumentado que su valor debe ser modificado en presencia de quarks dinámicos. De acuerdo con el análisis en la Ref. [114] se obtiene $T_{0}=210 \mathrm{MeV}$ para $N_{f}=2$ y $T_{0}=180 \mathrm{MeV}$ para $N_{f}=3$, con un error de aproximadamente $30 \mathrm{MeV}$. 
Hasta aquí tenemos por un lado el modelo de NJL para los quarks, y por otra parte un potencial para el PL que involucra al sector gluónico. El acoplamiento entre ellos se puede implementar utilizando la derivada covariante. Esto es, reemplazando en la acción euclídea

$$
\partial \rightarrow D_{\mu} \equiv \partial_{\mu}-i A_{\mu}
$$

con $A_{\mu}(x)=g \mathcal{A}_{\mu}^{a}(x) \lambda_{a} / 2$ donde $\mathcal{A}_{\mu}^{a}$ son los campos de gauge de $\mathrm{SU}(3), \mathrm{y} \lambda_{a}$ son las matrices de Gell-Mann.

En general el modelo supone que los quarks se mueven en presencia de un campo de color de fondo uniforme y estático, y por eso no se toma en cuenta la dependencia espacial del PL. Se considera entonces $A_{\mu}=\delta_{\mu}^{0} A_{0}$, con $A_{4}=i A_{0}=\varphi$. Además, trabajando en el llamado gauge de Polyakov, la matriz $A_{4}$ tiene representación diagonal

$$
A_{4}=\phi_{3} \lambda_{3}+\phi_{8} \lambda_{8}
$$

de manera que hay sólo dos variables independientes: $\phi_{3}$ y $\phi_{8}$. Entonces, la traza del PL $\Phi=\frac{1}{3} \operatorname{Tr} \exp \left[\frac{i}{T}\left(\phi_{3} \lambda_{3}+\phi_{8} \lambda_{8}\right)\right]$, que puede tomarse como parámetro de orden para el confinamiento, se puede escribir como

$$
\Phi=\frac{1}{3}\left[\exp \left(-\frac{2 i}{\sqrt{3}} \frac{\phi_{8}}{T}\right)+2 \exp \left(\frac{i}{\sqrt{3}} \frac{\phi_{8}}{T}\right) \cos \left(\frac{\phi_{3}}{T}\right)\right] .
$$

Debido a las propiedades de conjugación de carga del lagrangiano de QCD [115], $\Phi$ debe ser una cantidad real. Por ende si $\phi_{3}$ y $\phi_{8}$ son valores reales, esta condición implica que necesariamente $\phi_{8}=0$ [112], y la expresión anterior para la traza del loop de Polyakov se reduce a

$$
\Phi=\Phi^{*}=\frac{1}{3}\left[1+2 \cos \left(\frac{\phi_{3}}{T}\right)\right] .
$$

\subsection{Modelo PNJL no local con campo magnético a temperatura finita}

Extenderemos en esta sección el modelo introducido en el Cap. 5 a un sistema con temperatura finita. Partiendo de la ecuación (5.17) es posible estudiar las transiciones de fase y la dependencia con la temperatura de observables termodinámicos. Para ello, consideraremos el potencial termodinámico por unidad de volumen en la aproximación de campo medio (MFA) empleando el formalismo de Matsubara descrito en la Sec. 6.1 de este capítulo. También se incluirá el acoplamiento de los fermiones con el loop de Polyakov (PL) para considerar los efectos debido al confinamiento. Para ello trabajaremos con las hipótesis propuestas en la sección anterior, suponiendo que los los quarks se mueven en un campo de color de fondo constante y se utilizará el gauge de Polyakov, que conduce a la 
ecuación (6.37). De esta forma, el potencial termodinámico del sistema en presencia de un campo magnético externo está dado por

$$
\begin{aligned}
\Omega_{B, T}^{\mathrm{MFA}}= & \frac{\bar{\sigma}^{2}}{2 G}-T \sum_{n=-\infty}^{\infty} \sum_{c, f} \frac{\left|q_{f} B\right|}{2 \pi} \int \frac{d p_{3}}{2 \pi}\left[\ln \left(p_{\|_{n c}}^{2}+M_{0, p_{\|_{n c}}}^{\lambda, f}{ }^{2}\right)\right. \\
& \left.+\sum_{k=1}^{\infty} \ln \left(\Delta_{k, p_{\|_{n c}}}^{f}\right)\right]+\mathcal{U}(\Phi, T)
\end{aligned}
$$

donde hemos definido $p_{\|_{n c}}=\left(p_{3},(2 n+1) \pi T+\phi_{c}\right)$. Las sumas sobre los índices de color y sabor corren según $c=r, g, b$ y $f=u, d$, respectivamente, mientras que los campos de color de fondo son $\phi_{r}=-\phi_{g}=\phi_{3}, \phi_{b}=0$, es decir, $\phi_{c}=c \phi_{3}$ con $c=1,-1,0$ para $r, g$ y $b$.

Como es usual en los modelos no locales se puede observar que $\Omega^{\mathrm{MFA}}$ resulta ser divergente, y, por lo tanto, necesita ser regularizado. Utilizamos para ello una prescripción similar a la considerada por ejemplo en la Ref. [116], la cual consiste en sustraer la contribución del potencial termodinámico en ausencia de interacciones fermiónicas y luego sumarla en su forma regularizada. Siguiendo este procedimiento obtenemos una expresión para $\Omega_{B, T}^{\mathrm{MFA}, \mathrm{reg}}$

$$
\Omega_{B, T}^{\mathrm{MFA}, \mathrm{reg}}=\Omega_{B, T}^{\mathrm{MFA}}-\Omega_{B, T}^{\mathrm{free}}+\Omega_{B, T}^{\mathrm{free,reg}} .
$$

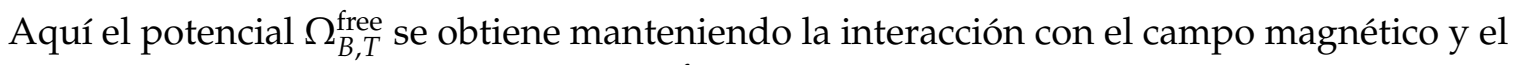
loop de Polyakov tomando $\bar{\sigma}=0$, y $\Omega_{\text {reg }}^{\text {free }}$ es la expresión regularizada para el potencial termodinámico de quarks en ausencia de los acoplamientos corriente-corriente. Para esta contribución la suma de Matsubara puede realizarse analíticamente, resultando

$$
\begin{aligned}
\Omega_{B, T}^{\text {free,reg }}= & -\frac{N_{c}}{2 \pi^{2}} \sum_{f}\left(q_{f} B\right)^{2}\left[\zeta^{\prime}\left(-1, x_{f}\right)+\frac{x_{f}^{2}}{4}-\frac{1}{2}\left(x_{f}^{2}-x_{f}\right) \log x_{f}\right] \\
& -T \sum_{f, c} \frac{\left|q_{f} B\right|}{\pi} \sum_{k=0}^{\infty} \alpha_{k} \int \frac{d p}{2 \pi} \log \left\{1+\exp \left[-\left(\epsilon_{k p}^{f}+i \phi_{c}\right) / T\right]\right\},
\end{aligned}
$$

donde $x_{f}=m_{c}^{2} /\left(2\left|q_{f} B\right|\right), \alpha_{k}=2-\delta_{k 0} \mathrm{y} \epsilon_{k p}^{f}=\left(2 k\left|q_{f} B\right|+p^{2}+m_{c}^{2}\right)^{1 / 2}$. A su vez, $\zeta^{\prime}\left(-1, x_{f}\right)=$ $d \zeta\left(z, x_{f}\right) /\left.d z\right|_{z=-1}$ donde $\zeta\left(z, x_{f}\right)$ es la función zeta de Hurwitz. A partir del potencial termodinámico se pueden determinar $\bar{\sigma}$ y $\phi_{3}$ resolviendo el sistema acoplado de ecuaciones de gap

$$
\frac{\partial \Omega_{B, T}^{\mathrm{MFA}, \mathrm{reg}}}{\partial \bar{\sigma}}=0, \quad \frac{\partial \Omega_{B, T}^{\mathrm{MFA}, \mathrm{reg}}}{\partial \phi_{3}}=0 .
$$

Para el estudio de las transiciones de fase resulta esencial analizar el comportamiento de los condensados quirales, debido a que éstos pueden utilizarse como parámetros de orden para las transiciones de fase de restauración de la simetría quiral, junto con la traza del loop de Polyakov que nos permitirá estudiar el deconfinamiento. Si estas transiciones son de primer orden, esto se verá reflejado en una discontinuidad en los parámetros de 
orden y la funciones termodinámicas. En cambio, si ocurren de manera suave (crossover), no existe una única manera de definir la temperatura de transición. Para estos casos será de utilidad definir las susceptibilidades correspondientes en función de la temperatura, lo cual hará posible tomar como temperaturas críticas a aquéllas en las que encontramos un máximo en dichas susceptibilidades.

En virtud de la expresión obtenida para el potencial $\Omega_{B, T}^{\mathrm{MFA}, \text { reg }}$ se calcula el condensado para cada sabor de quark derivando con respecto a la correspondiente masa corriente,

$$
\left\langle\bar{q}_{f} q_{f}\right\rangle_{B, T}^{\mathrm{reg}}=\frac{\partial \Omega_{B, T}^{\mathrm{MFA}, \mathrm{reg}}}{\partial m_{f}} .
$$

Esto conduce a la siguiente expresión para el condensado quiral

$$
\begin{aligned}
\left\langle\bar{q}_{f} q_{f}\right\rangle_{B, T}^{\mathrm{reg}}= & -\frac{\left|q_{f} B\right| T}{\pi} \sum_{c} \int \frac{d p_{3}}{2 \pi} \sum_{k=0}^{\infty} \sum_{n=-\infty}^{\infty}\left(\sum_{\lambda= \pm} \hat{A}_{k, p_{\|_{n c}}}^{\lambda, f}-\frac{2 m_{c}}{p_{\|_{n c}}^{2}+2 k\left|q_{f} B\right|+m_{c}^{2}}\right) \\
& -\frac{N_{c} m_{c}^{3}}{4 \pi^{2}}\left[\frac{\log \Gamma\left(x_{f}\right)}{x_{f}}-\frac{\ln 2 \pi}{2 x_{f}}+1-\left(1-\frac{1}{2 x_{f}}\right) \log x_{f}\right] \\
& +\frac{\left|q_{f} B\right|}{\pi} \sum_{c} \sum_{k=0}^{\infty} \alpha_{k} \int \frac{d p}{2 \pi} \frac{m_{c}}{\epsilon_{k p}^{f}} \frac{1}{1+\exp \left[\left(\epsilon_{k p}^{f}+i \phi_{c}\right) / T\right]} .
\end{aligned}
$$

La susceptibilidad quiral y la susceptibilidad asociada a la traza del PL pueden definirse ahora como

$$
\begin{aligned}
\chi_{\Phi} & =\frac{d \Phi}{d T}, \\
\chi_{c h} & =\frac{\partial\left\langle\bar{q}_{f} q_{f}\right\rangle_{B, T}^{\mathrm{reg}}}{\partial T},
\end{aligned}
$$

en donde la susceptibilidad quiral se encuentra promediada en los sabores $u$ y $d$.

$\mathrm{Al}$ igual que en la Sec. 5.1.1, definimos ahora el "condensado normalizado" $\Sigma_{B, T}^{f}$ (que en este caso depende también de la temperatura) como

$$
\Sigma_{B, T}^{f}=-\frac{2 m_{c}}{S^{4}}\left[\left\langle\bar{q}_{f} q_{f}\right\rangle_{B, T}^{\mathrm{reg}}-\left\langle\bar{q}_{f} q_{f}\right\rangle_{0,0}^{\mathrm{reg}}\right]+1
$$

Reintroducimos a su vez las definiciones $\Delta \Sigma_{B, T}^{f}=\Sigma_{B, T}^{f}-\Sigma_{0, T}^{f} \mathrm{y} \Delta \bar{\Sigma}_{B, T}=\left(\Delta \Sigma_{B, T}^{u}+\Delta \Sigma_{B, T}^{d}\right) / 2$.

\subsubsection{Resultados numéricos para temperatura finita}

En esta sección se presentan los resultados para las cantidades definidas en la subsección anterior, empleando los factores de forma introducidos en el Cap. 5 y los potenciales efectivos discutidos en la Sec. 6.1.3.

En los paneles superiores de la Figura 6.1 mostramos el comportamiento del condensado quiral promedio $\bar{\Sigma}_{B, T}$ y la traza del loop de Polyakov $\Phi$ como funciones de la temperatura, para tres valores representativos del campo magnético externo $B, e B=0,0,6 \mathrm{y}$ 


\begin{tabular}{l|cccc|cccc} 
& \multicolumn{4}{|c}{ Gaussiana } & \multicolumn{4}{c}{5 -Lorentziana } \\
$\left(-\langle q \bar{q}\rangle_{0,0}^{\text {reg }}\right)^{1 / 3}$ & 220 & 230 & 240 & 250 & 220 & 230 & 240 & 250 \\
\hline$T_{c}$ quiral & 182,1 & 179,1 & 177,4 & 176,6 & 177,0 & 177,0 & 177,8 & 179,5 \\
$T_{c}$ deconfinamiento & 182,1 & 178,0 & 175,8 & 174,6 & 174,8 & 174,7 & 175,5 & 176,8
\end{tabular}

Tabla 6.1: Temperaturas críticas de las transiciones de fase para la restauración de simetría quiral y el deconfinamiento con $B=0$ en varias parametrizaciones. Los valores de las temperaturas y de los condensados se encuentran expresados en $\mathrm{MeV}$.

$1 \mathrm{GeV}^{2}$. Las curvas corresponden a los parámetros que conducen a un condensado quiral $\left(-\langle\bar{q} q\rangle_{0,0}^{\mathrm{reg}}\right)^{1 / 3}=230 \mathrm{MeV}$, para un potencial de Polyakov polinómico con $T_{0}=210 \mathrm{MeV}$. Dado un valor de $B$, se puede observar que tanto para el caso del factor de forma gaussiano como el 5-lorentziano las transiciones de restauración de la simetría quiral y de deconfinamiento son de tipo crossover, y tienen lugar aproximadamente a la misma temperatura crítica. Por definición tomamos estas temperaturas como las correspondientes a los máximos de la susceptibilidades $\chi_{c h}$ y $\chi_{\Phi}$. Nuestros resultados para el comportamiento de las susceptibilidades como funciones de la temperatura, para $B=0,0.6$ y $1 \mathrm{GeV}^{2}$, se muestran en los paneles inferiores de la Figura 6.1.

Las temperaturas críticas de restauración de simetría quiral y deconfinamiento obtenidas en ausencia de campo magnético para diferentes parametrizaciones se encuentran en la Tabla 6.1. Se puede observar que para todos los casos la separación entre ambas temperaturas críticas es menor a los $5 \mathrm{MeV}$, lo cual es consistente con los resultados obtenidos en LQCD. De los resultados mostrados en esta tabla podemos ver también que los valores de las temperaturas críticas no varían significativamente con las parametrizaciones (recordemos que en todos los casos los parámetros fueron ajustados de forma tal de reproducir los valores empíricos de la masa y la constante de decaimiento débil del pion). Por otra parte, estas temperaturas críticas son ligeramente mayores que las obtenidas en LQCD, que rondan el valor de $160 \mathrm{MeV}$. En realidad, el valor de $T_{c}$ y la pendiente de la transición dependen de la forma del potencial de Polyakov. En particular, para un potencial de Polyakov polinómico podemos obtener $T_{c} \simeq 160 \mathrm{a} 165 \mathrm{MeV}$, dependiendo de la parametrización. El potencial logarítmico en la ecuación (6.28) conduce a transiciones más abruptas, las cuales pueden ser incluso de primer orden para ciertos valores de los parámetros. Vale la pena notar que en ausencia de interacción con el loop de Polyakov los valores de $T_{\mathcal{c}}$ bajan a $130 \mathrm{MeV}$ [90].

Para analizar el efecto de los campos magnéticos en la transición de fase vemos que en la Figura 6.1 la separación de las temperaturas críticas de restauración de simetría quiral 

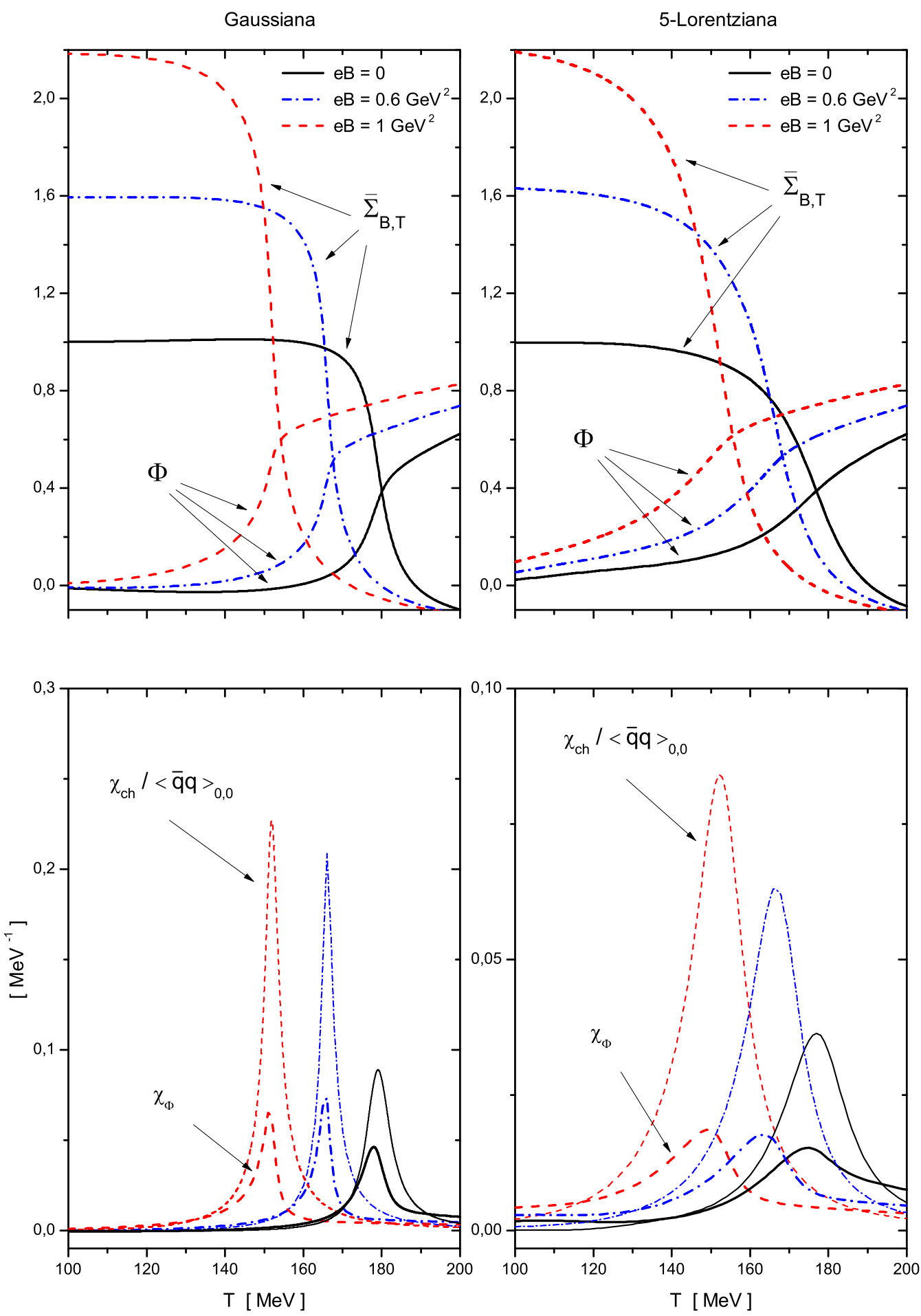

Figura 6.1: Izquierda: Condensado sustraído normalizado promediado en sabor como función de $e B$ para diferentes temperaturas representativas. Derecha: condensado normalizado promediado en sabor como función de la temperatura para diferentes valores representativos de $e B$. Los resultados en ambos paneles corresponden a $\left(-\langle\bar{q} q\rangle_{0,0}^{\text {reg }}\right)^{1 / 3}=230 \mathrm{MeV}$. 
y deconfinamiento se mantiene pequeña en presencia del campo externo (de hecho, un análisis detallado muestra que la separación se reduce a medida que aumenta $e B$ ). Además, podemos ver que el modelo NJL no local muestra el efecto conocido como Catálisis Magnética Inversa, es decir que, al contrario de lo que ocurre en el modelo NJL local estándar [85, 86, 87], en nuestro modelo la temperatura crítica de la restauración quiral es cada vez menor a medida que el campo magnético externo aumenta. Esto se relaciona con el hecho de que, en general, los condensados no crecen monótonamente con $B$ para un dado valor fijo de temperatura. Esta situación se ve representada en la Figura 6.2, donde se muestra el comportamiento del parámetro $\Delta \bar{\Sigma}_{B, T}$ como función de $e B$ para el caso de $T=0$ y para los valores de temperatura cercanos a la temperatura de transición. Las curvas en los paneles izquierdo y derecho corresponden al modelo con factores de forma gaussianos y lorentzianos, respectivamente, con la parametrización correspondiente al condensado $\left(-\langle\bar{q} q\rangle_{0,0}^{\mathrm{reg}}\right)^{1 / 3}=230 \mathrm{MeV}$, y potencial de Polyakov polinómico. Para estas parametrizaciones las temperaturas críticas para $B=0$ se ubican ligeramente por debajo de los $180 \mathrm{Mev}$ (ver la Tabla 6.1). A pesar de que para $T=0$ el valor de $\Delta \bar{\Sigma}_{B, 0}$ crece monótonamente con el campo magnético externo, podemos ver que, a medida que las temperaturas se acercan a los valores críticos, las curvas presentan un máximo y luego comienzan a decrecer a medida que aumenta el campo. Finalmente, en la Figura 6.3 se presentan los gráficos de las temperaturas críticas de la restauración quiral $T_{c}(B)$, normalizadas con los valores correspondientes a campo magnético nulo. La figura incluye las curvas para los modelos NJL no locales con factores de forma gaussianos (izquierda) y 5-lorentzianos (derecha) y diferentes paramerizaciones. Las bandas grises en ambos paneles corresponden a los resultados obtenidos en LQCD, tomados de la Ref. [84]. Las líneas gruesas para ambos factores de forma corresponden a los potenciales de PL polinómicos según la ecuación (6.31). Se omitieron los resultados para el potencial logarítmico debido a que en ese caso las transiciones son muy abruptas comparadas con los resultados de LQCD. Podemos observar en la figura que la catálisis magnética inversa ocurre para todas las parametrizaciones consideradas. Para ambos factores de forma, se encuentra que el efecto es más intenso para las parametrizaciones correspondientes a los condensados quirales de menor valor absoluto, y también depende de los potenciales de Polyakov. Como una conclusión general, podemos decir que el comportamiento de las temperaturas críticas con el campo magnético es compatible con los resultados de LQCD para valores adecuados de los condensados quirales.

El mecanismo que conduce a la catálisis magnética inversa en el contexto de los modelos no locales se puede comprender notando que las transformadas de los factores de forma no locales son funciones del campo magnético externo. Esto se puede ver claramente en la ecuación (5.12). Recordando que en el modelo NJL no local los factores de forma representan interacciones efectivas mediadas por un gluón de rango finito, el origen de la 

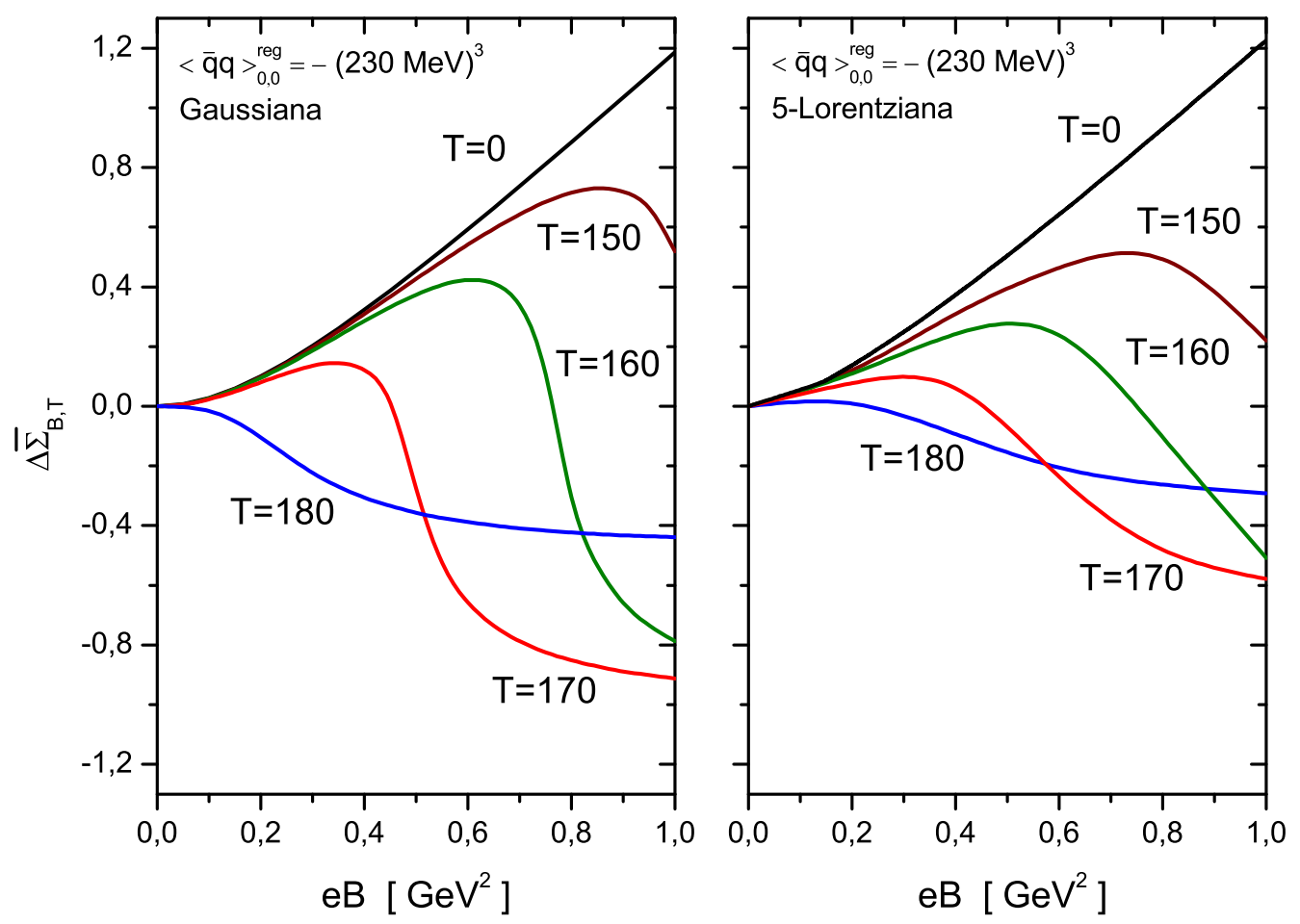

Figura 6.2: Izquierda: condensado sustraído promedio en sabor como función de $e B$ para diferentes valores representativos de la temperatura. Los paneles derecho e izquierdo corresponden a los factores de forma gaussiano y 5-lorentziano respectivamente. Los resultados es ambos paneles corresponden a $\left(-\langle\bar{q} q\rangle_{0,0}^{\text {reg }}\right)^{1 / 3}=230 \mathrm{MeV}$.
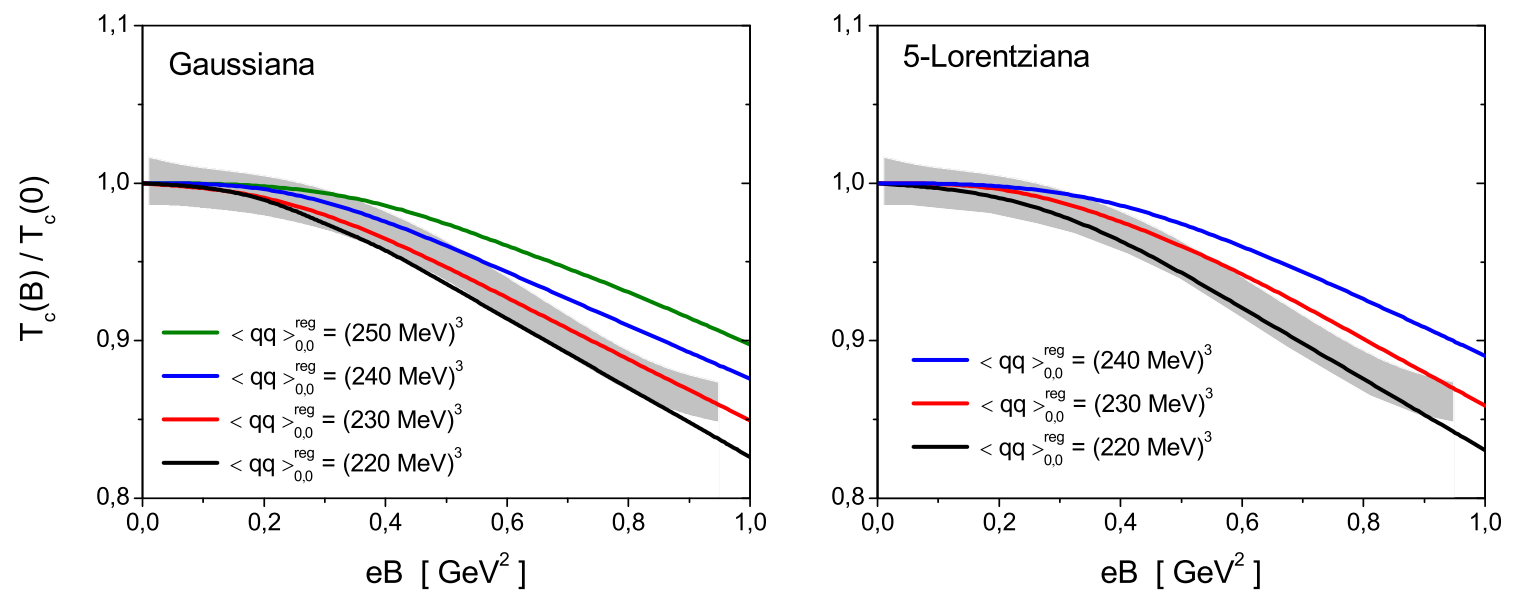

Figura 6.3: Temperaturas de restauración de la simetría quiral normalizadas como función de $e B$ para varias parametrizaciones del modelo. Para comparación se incluyen en la banda gris los resultados de LQCD presentados en Ref. [84]. Los paneles derecho e izquierdo corresponden a los factores de forma gaussiano y 5-lorentziano respectivamente. 
dependencia del factor de forma con el campo magnético puede interpretarse como debido a una acción de respuesta de los quarks sobre los campos gluónicos. Es interesante considerar la masa efectiva para el caso particular del factor de forma gaussiano, dada por la ecuación (5.88). Se puede ver que en este caso las componentes del momento que son paralelas y transversas al campo magnético se desacoplan. Mientras que las componentes 3 y 4 de la forma exponencial original $\exp \left(-p^{2} / \Lambda^{2}\right)$ permanecen allí, las componentes transversas 1 y 2 conducen a un factor dado por un cociente de polinomios en $\left|q_{f} B\right| / \Lambda^{2}$, que tiende a cero cuando $B$ es muy grande. De este modo, para cualquier nivel de Landau $k$ la intensidad del acoplamiento efectivo decrece a medida que $e B$ aumenta. Esto es análogo a lo que ocurre con los modelos en donde se proponen constantes de acoplamiento dependientes del campo $B$ (ver por ejemplo las Refs. $[89,117]$ ). 



\section{Capítulo 7}

\section{Resumen y conclusiones}

A lo largo de esta Tesis Doctoral se han estudiado distintos aspectos del comportamiento de la materia fuertemente interactuante en el marco de modelos de quarks relativistas que incluyen interacciones de tipo no local. Como aspectos principales de la investigación original realizada se destacan en primer lugar el análisis de la fenomenología asociada con el sector de mesones vectoriales y vectoriales-axiales, y en segundo lugar el estudio de los efectos de campos magnéticos intensos sobre la materia de quarks, en particular, los efectos de Catálisis Magnética y Catálisis Magnética Inversa. También se analizó el comportamiento, en presencia de estos campos, de la masa y constante de decaimiento de los mesones $\pi$.

Comenzamos presentando una introducción general en la que se detallan los objetivos de esta Tesis. En el Cap. 2 introdujimos la noción de simetría dentro del Modelo Estándar y repasamos algunos conceptos esenciales de la Cromodinámica Cuántica (QCD), enfocándonos en aquéllos relacionados con la ruptura espontánea de las simetrías vinculadas a las transiciones de fase. Luego, en el Cap. 3 introdujimos los modelos efectivos como una herramienta para estudiar la fenomenología de los hadrones a bajas energías. En particular consideramos el modelo de Nambu y Jona-Lasinio (NJL) mostrando que permite describir adecuadamente la ruptura espontánea de la simetría quiral y la aparición de los pseudobosones de Goldstone identificados con los mesones $\pi$. Finalmente notamos que es posible obtener una descripción más realista de las interacciones entre quarks teniendo en cuenta interacciones de tipo no local.

En estos tres primeros capítulos presentamos las bases fundamentales para lo que constituye el aporte original de esta Tesis, que puede dividirse en tres partes. En el Cap. 4 analizamos un modelo de quarks con interacciones no locales con simetría SU(2) de sabor que incluyen acoplamientos entre corrientes vectoriales y vectoriales-axiales, fijando los parámetros libres de la teoría, es decir, las constantes de acoplamiento, la masa corriente de los 
quarks y los factores de forma. En el Cap. 5 consideramos en este marco teórico general sistemas hadrónicos en presencia de campos magnéticos externos intensos, estudiando la Catálisis Magnética, y determinando el efecto de la presencia de dichos campos sobre la masa y la constante de decaimiento débil del pion. Finalmente, en el Cap. 6 analizamos el efecto conocido como Catálisis Magnética Inversa estudiando las transiciones de fase quiral y de deconfinamiento en función de la temperatura para sistemas sometidos a campos magnéticos externos.

El análisis del Cap. 4 está centrado en una extensión del modelo NJL que incluye interacciones no locales de cuatro fermiones y un acoplamiento entre las llamadas "corrientes de momento" que conduce a la renormalización de la función de onda (WFR) de los quarks. Mostramos cómo a partir de este modelo se obtiene un propagador de quark "vestido", en el cual la masa efectiva y la WFR son funciones del momento del quark a través de factores de forma no locales. Mostramos también que el modelo permite ajustar estos factores de forma de modo tal de reproducir resultados obtenidos en cálculos de LQCD. Para el tratamiento del modelo se recurrió al formalismo de bosonización, por medio del cual se reescribe la función de partición del sistema en término de campos mesónicos, integrando los grados de libertad fermiónicos. Trabajando en la aproximación de campo medio, obtuvimos expresiones para las masas dinámicas de los quarks y el condensado quiral $\langle\bar{\psi} \psi\rangle$, el cual es utilizado como parámetro de orden de la transición de fase asociada con la ruptura espontánea de la simetría quiral. Desarrollando la acción efectiva a segundo orden en las fluctuaciones de los campos, determinamos las masas de los mesones escalares, pseudoescalares, vectoriales y vectoriales-axiales, y a su vez analizamos la mezcla en el sector $\pi-\mathrm{a}_{1}$ encontrando una prescripción que nos permitió definir los campos bosónicos $\tilde{\vec{\pi}}$ y $\tilde{\vec{a}}_{\mu}$ desacoplados correspondientes a los estados físicos de estos mesones. Por otro lado, considerando acoplamientos con corrientes externas a través de la derivada covariante, obtuvimos expresiones para anchos parciales de decaimiento débil y electromagnético de los mesones $\pi$, y para el decaimiento del mesón $\rho^{0}$ en dos leptones. Encontramos que, debido al carácter no local de las interacciones, el modelo incluye, además de las contribuciones de loops de quarks usuales, la contribución de diagramas tipo tadpole que no se encuentran presentes en el modelo NJL local. Se encontró también que para el decaimiento electromagnético del mesón $\pi^{0}$, a pesar de que las contribuciones individuales de los diagramas resultan divergentes, al sumar el total de las contribuciones dichas divergencias se cancelan encontrando así resultados finitos. Algo similar ocurre para el caso del decaimiento de mesón $\rho^{0}$, para el que se obtienen contribuciones de naturaleza longitudinal que se cancelan entre ellas al momento de realizar la suma total, que resulta transversa como lo requiere la conservación de la corriente electromagnética. Por último, se llevó a cabo una extensión del desarrollo de la acción euclídea hasta el orden cúbico para calcular el ancho de decai- 
miento $\rho \rightarrow \pi \pi$. Consideramos una parametrización para las interacciones determinada a partir del ajuste de las funciones de masa $M(p)$ y renormalización de la función de onda $Z(p)$ de quarks a resultados obtenidos en LQCD. Los parámetros libres de la teoría, es decir, la masa corriente de los quarks y las constantes de acoplamiento corriente-corriente, se determinaron a partir de observables fenomenológicos, eligiendo como inputs valores empíricos de masas de mesones y la constante de decaimiento débil del pion. A partir de la evaluación numérica de las expresiones analíticas, para la cual se debió introducir una prescripción que permite tratar con las integrales a un loop, encontramos que el modelo es capaz de reproducir los valores empíricos de los observables calculados, y conduce a valores fenomenológicamente aceptables para las masas y los anchos de decaimiento. Esto incluye predicciones satisfactorias para los anchos de desintegración $\pi^{0} \rightarrow \gamma \gamma, \rho \rightarrow \pi \pi \mathrm{y}$ $\rho \rightarrow e^{+} e^{-}$, y para la masa de la resonancia $a_{1}(1260)$.

En los Caps. 5 y 6 nos enfocamos en las propiedades de la materia hadrónica en presencia de campos magnéticos intensos en el contexto de los modelos con interacciones no locales. En el Cap. 5 comenzamos por incluir en la acción efectiva con acoplamientos corriente-corriente no locales escalares y pseudoecalares un acoplamiento a un campo electromagnético externo uniforme. Definimos entonces una derivada covariante dependiente de la carga de los quarks up y down, la cual rompe la simetría de isospín. Utilizamos el método de autofunciones de Ritus para obtener una expresión de la acción efectiva diagonal en el espacio de sabor, y en la aproximación de campo medio encontramos la ecuación del gap y obtuvimos expresiones analíticas para los condensados quirales de quarks y para los propagadores de quarks en presencia del campo magnético. Desarrollando la acción efectiva a segundo orden en los campo mesónicos, y utilizando dicho propagador, logramos luego de un largo cálculo analítico encontrar expresiones para las masas de los mesones $\pi$ y $\sigma$, y para la constante de decaimiento del pion. Para el factor de forma no local se consideraron dos formas funcionales: en primer lugar utilizamos funciones gaussianas (con las que el cálculo resulta más simple), y en segundo lugar utilizamos la función denominada 5-lorentziana. En ambos casos se consideraron varios conjuntos de parámetros fenomenológicamente aceptables.

A partir del análisis numérico se estudió el comportamiento de los condensados promediados, el cual resultó compatible con el efecto de catálisis magnética, esto es, se encontró que el condensado quiral tiende a estabilizarse en la fase hadrónica al aplicarse un campo magnético externo. Tal como sucede en otros modelos, nuestras predicciones concuerdan cuantitativamente con los resultados de LQCD. En los cálculos de las masas de los mesones livianos se observa que éstas disminuyen a medida que el campo magnético es más intenso, mientras que la constante de decaimiento débil del pión muestra el comportamiento opuesto, aumentando su valor con el campo. 
En el Cap. 6, siempre en el marco de los modelos de quarks no locales, comenzamos describiendo el formalismo necesario para estudiar sistemas hadrónicos a temperatura finita y mostramos cómo acoplar los grados de libertad fermiónicos al loop de Polyakov en lo que llamamos "modelo nlPNJL". Presentamos dos formas alternativas para el término de gauge puro en el potencial termodinámico: una polinómica basada en el ansatz de Ginzgurg-Landau y otra basada en la forma logarítmica de la medida de Haar de integración asociada con el grupo SU(3) de color. En la Sección 6.2 incorporamos al modelo descrito en el Cap. 5 el formalismo de temperatura finita y así obtuvimos un modelo para estudiar las temperaturas críticas y transiciones de fase de restauración de la simetría quiral y deconfinamiento en presencia de campos magnéticos. Utilizando las mismas parametrizaciones descritas anteriormente obtuvimos que a temperaturas intermedias el condensado quiral disminuye a medida que aumenta la intensidad del campo magnético. Estudiando las curvas para la transición de fase en función de la temperatura, notamos que el modelo predice una disminución de la temperatura crítica de transición de fase quiral al incrementarse el campo magnético externo. Este comportamiento, conocido como Catálisis Magnética Inversa, ha sido también obtenido a partir de cálculos realizados mediante LQCD. Sin embargo, sólo ha sido posible reproducirlo en el marco de modelos efectivos como el NJL local introduciendo una dependencia ad hoc de las constantes de acoplamiento con el campo magnético. Nuestros resultados muestran que los modelos que incluyen interacciones no locales conducen naturalmente a la Catálisis Magnética Inversa, lo cual puede entenderse teniendo en cuenta que debido a la no localidad los acoplamientos corriente-corriente resultan ser dependientes de la temperatura y el campo magnético a través de los factores de forma, que de este modo actúan como interacciones magnéticas efectivas. Esta dependencia surge del proceso analítico para el cálculo de la acción efectiva, sin introducir dependencias ad hoc en las constantes de acoplamiento u otras formas utilizadas en modelos alternativos para reproducir este efecto.

Para obtener una descripción más realista de la termodinámica de las interacciones fuertes, y el cálculo del diagrama de fases de QCD, es importante la inclusión de la WFR y el potencial químico. Es nuestro objetivo continuar con este desarrollo incorporando estos nuevos elementos al modelo en presencia de campos magnéticos, de modo tal de avanzar en el estudio de las propiedades de la materia hadrónica y poder abarcar, por ejemplo, el análisis de estrellas compactas.

En lo que hace a la fenomenología de los mesones, concluimos que la inclusión del sector de mesones vectoriales y axiales ofrece una visión más completa de la fenomenología de los mesones livianos, y sus efectos son importantes para el análisis de observables hadrónicos. Dada la importancia del mesón $\rho$ en las colisiones de iones pesados, planeamos a futuro extender el estudio de las propiedades del mesón $\rho$ a sistemas con temperatura 
finita. Además, para el caso de sistemas hadrónicos a potencial químico finito se espera que las interacciones vectoriales conduzcan a un condensado distinto de cero en el canal " $\omega$ " $\left(J^{C P}=1^{1--}, I=0\right)$, el cual es importante para el estudio del diagrama de fase de QCD [118] y la física de estrellas compactas [119]. 



\section{Apéndice A}

\section{Bosonización}

El término bosonización de una teoría fermiónica refiere a la transformación del lagrangiano fermiónico original en uno equivalente que únicamente dependa de grados de libertad bosónicos. Una transformación de este tipo sólo puede realizarse en algunos casos. En el modelo NJL el lagrangiano bosónico correspondiente puede ser construido solamente en forma aproximada, y la acción bosónica efectiva resultante refleja la dinámica de la acción original del modelo en el régimen de bajas energías.

La ventaja de este método es que provee relaciones entre observables físicos como constantes de acoplamiento, condensados, etc. Es una formulación conveniente para discutir características importantes del modelo a bajas energías, y además permite realizar comparaciones con lagrangianos fenomenológicos de mesones quirales.

Para el caso del modelo no local con dos sabores de quarks y con WFR, consideramos la función de partición correspondiente $\mathcal{Z}=\int \mathcal{D} \psi \mathcal{D} \psi \exp \left[-S_{E}\right]$, e introducimos los campos mesónicos $\sigma_{1,2}(x)$ y $\vec{\pi}(x)$ (escalares y pseudoescalares respectivamente), junto con campos auxiliares $S_{1,2}(x)$ y $\vec{P}(x)$. Esto puede hacerse mediante el uso de las igualdades

$$
\begin{aligned}
F\left[j_{S}(x), j_{P}^{a}(x)\right]= & \int \mathcal{D} \sigma_{1} \mathcal{D} \pi^{a} \mathcal{D} S_{1} \mathcal{D} P^{a} F\left[S_{1}(x), P^{a}(x)\right] \times \\
& \exp \left(\int d^{4} x\left\{\sigma_{1}(x)\left[S_{1}(x)-j_{S}(x)\right]+\pi^{a}(x)\left[P^{a}(x)-j_{P}^{a}(x)\right]\right\}\right),
\end{aligned}
$$

y

$$
F\left[j_{M}(x)\right]=\int \mathcal{D} \sigma_{2} \mathcal{D} S_{2} F\left[S_{2}(x)\right] \times \exp \left(\int d^{4} x\left\{\sigma_{2}(x)\left[S_{2}(x)-j_{M}(x)\right]\right\}\right) .
$$

Reemplazando en la acción euclídea $S_{E}$ dada en la ecuación (3.25), obtenemos una expresión dependiente de los campos fermiónicos, así como de los nuevos campos bosónicos que introdujimos. La integral sobre los campos fermiónicos puede resolverse analíticamente a través de la propiedad

$$
\int \mathcal{D} \bar{\psi} \mathcal{D} \psi \exp \left[d^{4} x_{1} d^{4} x_{2} \bar{\psi}\left(x_{1}\right) A\left(x_{1}, x_{2}\right) \psi\left(x_{2}\right)\right]=\operatorname{det} A\left(x_{1}, x_{2}\right) .
$$


Una vez realizada esta integral, la expresión para la función de partición resulta

$$
\begin{aligned}
\mathcal{Z}= & \int \mathcal{D} \sigma_{1} \mathcal{D} \vec{\pi} \mathcal{D} \sigma_{2} \exp \left[\log \operatorname{det} A\left(\sigma_{1}, \vec{\pi}, \sigma_{2}\right)\right] \times \\
& \int \mathcal{D} S_{1} \mathcal{D} \vec{P} \mathcal{D} S_{2} \exp \left[\sigma_{1} S_{1}+\vec{\pi} \cdot \vec{P}+\sigma_{2} S_{2}+\frac{G_{S}}{2}\left(S_{1} S_{1}+\vec{P} \cdot \vec{P}+S_{2} S_{2}\right)\right] .
\end{aligned}
$$

Por último, se integran los campos auxiliares $S_{1,2}(x)$ y $\vec{P}(x)$, y se realiza una transformación de Fourier pasando todo al espacio de momentos. De ese modo se obtiene la expresión de la acción bosonizada en la ecuación (3.28), con el operador $A\left(p, p^{\prime}\right)$ dado por la ecuación (3.29). 


\section{Apéndice B}

\section{Integrales a un loop y factores de forma en el plano complejo}

Como describimos en la Sec. 4.3, consideramos una parametrización para el modelo NJL no local que nos permite reproducir los resultados obtenidos por LQCD para la dependencia del propagador efectivo de los quarks con el momento. De la comparación con los datos de LQCD, los factores de forma $g(p)$ y $f(p)$ fueron escritos en términos de las funciones $f_{m}\left(p^{2}\right)$ y $f_{z}\left(p^{2}\right)$ dadas por las ecuaciones (4.53) y (4.55). En este apéndice discutiremos la evaluación numérica de las integrales a un loop, que deben ser tratadas con precaución dada la forma funcional de $f_{m}\left(p^{2}\right)$.

Consideremos integrales a un loop que involucran un momento externo $p$, como es el caso para las funciones $G_{M}\left(p^{2}\right), F_{0,1}\left(p^{2}\right)$ y $J^{(\mathrm{I}, \mathrm{II})}\left(p^{2}\right)$, definidas en las Secs. 4.1.2 y 4.2. Las integrales pueden expresarse genéricamente como

$$
I\left(p^{2}\right)=\int \frac{d^{4} q}{(2 \pi)^{4}} F\left(q^{+}, q^{-}, p\right),
$$

donde $q^{ \pm}=q \pm p / 2$, y $F\left(q^{+}, q^{-}, p\right)$ es una función que incluye los factores de forma explícitamente o a través de las masas efectivas de los quarks y/o de las renormalizaciones de las funciones de onda. Más precisamente, se observa que en general $F\left(q^{+}, q^{-}, p\right)$ puede incluir los factores de forma $f_{m}(s)$ evaluados en $s=\left(q^{+}\right)^{2},\left(q^{-}\right)^{2}$ y $/ \mathrm{o} q^{2}$. Estamos interesados en esta función ya que su forma explícita $f_{m}(s)=1 /\left[1+\left(s / \Lambda_{0}^{2}\right)^{3 / 2}\right]$ implica la existencia de un corte en el campo complejo $s$, en $\operatorname{Re}(s)<0, \operatorname{Im}(s)=0$. Es conveniente destacar que en todos los casos las integrales deben ser evaluadas numéricamente en $p^{2}=-M^{2}$, donde $M$ es la masa de alguna partícula mesónica.

Para realizar los cálculos escogemos, como se realiza usualmente, el eje 4 en la dirección del momento externo, obteniendo así $p^{\mu}=(i M, \overrightarrow{0})$, de modo que $I\left(p^{2}\right)$ se reduce a una integral doble en $q_{4}$ y $|\vec{q}|$. En virtud de la simetría de las funciones $F\left(q^{+}, q^{-}, p\right)$ ante el 
intercambio $q^{+} \leftrightarrow q^{-}$, es sencillo concluir que $F\left(q^{+}, q^{-}, p\right)=F\left(q^{+*}, q^{-*}, p\right)$, lo que asegura que $I\left(q^{2}\right)$ sea real.

Ahora tomamos un $|\vec{q}|$ fijo y consideramos la estructura analítica del integrando en el plano complejo $q_{4}$. Inmediatamente podemos identificar dos cortes en este plano que surgen de la función $f_{m}\left(q^{2}\right)$, y otros pares de cortes aparecerán por la presencia de $f_{m}\left[\left(q^{+}\right)^{2}\right]$ y $f_{m}\left[\left(q^{-}\right)^{2}\right]$, respectivamente. En el caso de $f_{m}\left(q^{2}\right)=f_{m}\left(q_{4}^{2}+|\vec{q}|^{2}\right)$, los cortes están dados por $\operatorname{Re}\left(q_{4}\right)=0,\left|\operatorname{Im}\left(q_{4}\right)\right|>|\vec{q}|$, y por lo tanto estos nunca cruzarán el eje real $q_{4}$, sobre el cual debe realizarse la integral. Por otro lado, para $f_{m}\left[\left(q^{ \pm}\right)^{2}\right]$ los cortes se encuentran en $\operatorname{Re}\left(q_{4}\right)=0,\left|\operatorname{Im}\left(q_{4}\right) \pm M / 2\right|>|\vec{q}|$, y entonces, si $|\vec{q}|<M / 2$, tanto $f_{m}\left[\left(q^{+}\right)^{2}\right]$ como $f_{m}\left[\left(q^{-}\right)^{2}\right]$ presentarán cortes que en efecto atravesarán el eje real $q_{4}$.

El modo de tratar estos cortes es una cuestión de prescripción. De hecho, luego de tomar los factores de forma de los cálculos de LQCD en el espacio Euclídeo se puede volver al espacio de Minkowski a través de una rotación de Wick. Entonces se encontraría que los cortes se ubican sobre el eje de integración, y para evaluar las integrales éstas deberían ser modificadas siguiendo alguna receta. En nuestro caso tomamos la prescripción de trasladar los argumentos de $f_{m}(s)$ de acuerdo con

$$
\begin{aligned}
& f_{m}\left[\left(q^{+}\right)^{2}\right] \rightarrow f_{m}\left[\left(q^{+}\right)^{2}-i \varepsilon\right], \\
& f_{m}\left[\left(q^{-}\right)^{2}\right] \rightarrow f_{m}\left[\left(q^{-}\right)^{2}+i \varepsilon\right],
\end{aligned}
$$

mientras que $f_{m}\left(q^{2}\right)$ no se modifica. De este modo, los cortes no se superponen entre ellos y la propiedad $F\left(q^{+}, q^{-}, p\right)=F\left(q^{+*}, q^{-*}, p\right)$ se mantiene válida. A partir de las ecuaciones (B2) y (B3) los cortes asociados a las funciones $f_{m}\left[\left(q^{ \pm}\right)^{2}\right]$ estarán dados por

$$
\left\{\begin{aligned}
\operatorname{Re}\left(q_{4}\right)-\frac{\varepsilon}{M \pm 2 \operatorname{Im}\left(q_{4}\right)} & =0, \\
\left|\operatorname{Im}\left(q_{4}\right) \pm M / 2\right|-|\vec{q}| & >0 .
\end{aligned}\right.
$$

Las curvas correspondientes en el plano $q_{4}$ se pueden observar en la Figura B.1, donde hemos distinguido dos situaciones: la primera en donde $|\vec{q}|>M / 2$ (Figura B.1a), y la segunda cuando $|\vec{q}|<M / 2$ (Figura B.1b). Los cortes correspondientes a las funciones $f_{m}\left[\left(q^{+}\right)^{2}\right]$, $f_{m}\left[\left(q^{-}\right)^{2}\right]$ y $f_{m}\left(q^{2}\right)$ han sido representados con líneas discontinuas, de puntos (azul), trazos (rojo) y puntos y trazos (negro), respectivamente. En la primera situación $(|\vec{q}|>M / 2)$ los cortes no cruzan el eje de integración, por lo tanto no hay contribuciones extras a la integral. En el segundo caso, para $|\vec{q}|<M / 2$, dos cortes cruzan de un semiplano al otro, pasando a través del eje real $q_{4}$. Como la integral sobre $q_{4}$ debe ser equivalente a una integral sobre el momento $q_{0}$ de Minkowski, obtenida a través de su correspondiente rotación de Wick, el contorno de integración sobre $q_{4}$ debería deformarse de forma tal de sustraer las contribuciones de las partes que cruzan, que se encuentran representadas por las líneas 
sólidas (azul y roja) en la Figura B.1b. Un procedimiento similar se debe emplear cuando polos del integrando cruzan el eje de integración a cierto valor de $|\vec{q}|$; en ese caso las contribuciones resultantes de la deformación del contorno de integración de $q_{4}$ se pueden obtener calculando los residuos de los polos, de acuerdo al teorema de Cauchy. La necesidad de agregar contribuciones de polos o cortes a las integrales de loop se vuelve evidente observando las integrales relativamente sencillas que aparecen, por ejemplo, en las ecuaciones de gap (3.38): si se realiza una traslación del momento del loop $p \rightarrow p^{\prime}=p+r$, con $r^{2}=-M^{2}$, para un valor fijo de $\left|\vec{p}^{\prime}\right|$ aparecerán cortes en el plano complejo $p_{4}^{\prime}$ que cruzan del semiplano superior al inferior (o vice-versa). Además, en general el integrando tendrá polos que para valores suficientemente grandes de $M$ cruzan el eje real $p_{4}^{\prime}$ en algún valor de $\left|\vec{p}^{\prime}\right|$. Partiendo del teorema de Cauchy es sencillo calcular las contribuciones correspondientes, que deben ser sustraídas si se requiere que la integral a un loop sea invariante ante la traslación.

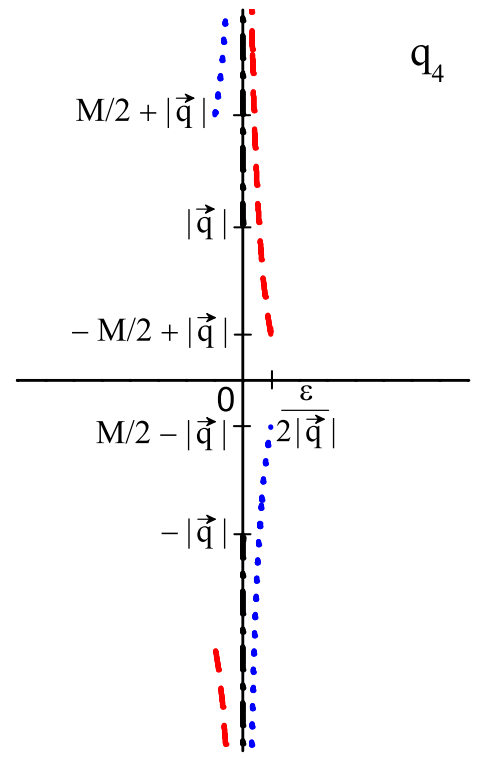

( a )

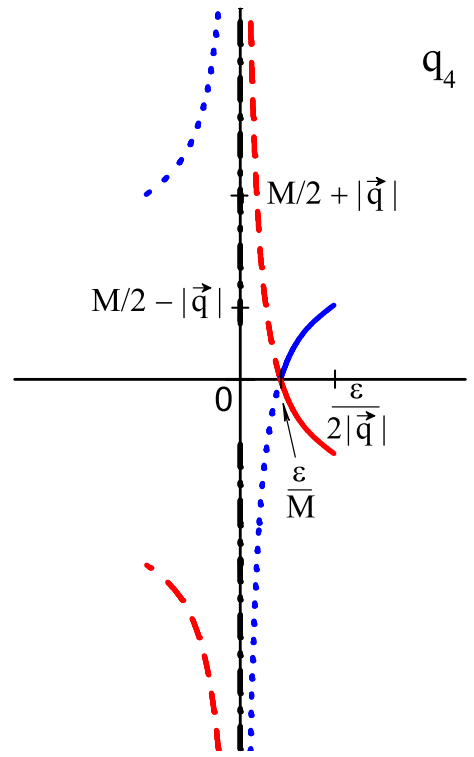

( b )

Figura B.1: Cortes de las funciones $F\left(q^{+}, q^{-}, p\right)$ en el plano complejo $q_{4}$, de acuerdo con la prescripción dada por las ecuaciones (B2) y (B3). Las curvas en los gráficos (a) y (b) corresponden a $|\vec{q}|>M / 2$ y $|\vec{q}|<M / 2$, respectivamente.

En la práctica las contribuciones de los cortes pueden obtenerse realizando integrales en el plano $q_{4}$ sobre contornos adecuados que encierren los trozos que cruzan el eje y luego tomando $\varepsilon \rightarrow 0$. Debido a la simetría de las funciones $F\left(q^{+}, q^{-}, p\right)$ las partes imaginarias 
de las integrales en los semiplanos superior e inferior se cancelan entre sí, obteniéndose una contribución total real. Luego, el resultado debe ser integrado sobre el momento tridimensional $|\vec{q}|$. Hay que destacar que - de acuerdo con las condiciones en (B4) - esta integración tiene como límites $|\vec{q}|=0$ y $|\vec{q}|=M / 2$, de modo que la contribución de los cortes resultará despreciable si la masa del mesón $M$ es relativamente pequeña, lo cual ocurre en general para el caso $M=m_{\pi}$.

Finalmente, en el caso del factor de forma para el decaimiento $\rho \rightarrow \pi \pi$ la situación es más complicada debido a que la integral relevante, dada por (4.50), involucra dos momentos externos independientes $p$ y $v$. Con el mismo procedimiento se encuentra que el integrando en este caso tiene dos cortes adicionales en el plano complejo $q_{4}$ que surgen de las funciones $f_{m}(s)$ evaluadas en $s=(q+v / 2 \pm p / 4)^{2}$. Para tratar con estos cortes hemos utilizado la prescripción $f_{m}\left[(q+v / 2 \pm p / 4)^{2}\right] \rightarrow f_{m}\left[(q+v / 2 \pm p / 4)^{2} \pm i \varepsilon^{\prime}\right]$, eligiendo un camino de integración que encierre los trozos de los cortes que cruzan el eje real $p_{4}$ del modo explicado previamente. 


\section{Apéndice $\mathrm{C}$}

\section{Autofunciones y transformaciones de Ritus}

En este apéndice proveeremos la forma explícita de las funciones de Ritus [102] y discutiremos algunas de sus propiedades. Estas funciones son en realidad matrices de $4 \times 4$ que satisfacen la ecuación de autovalores

$$
\Pi^{2} \mathbb{E}_{\bar{p}}(x)=\epsilon_{\bar{p}} \mathbb{E}_{\bar{p}}(x)
$$

donde $\Pi$ es el operador definido en el Cap. 5, $\Pi=-i \not \supset-q B x_{1} \gamma_{2}$. Aquí, $\bar{p}=\left(k, p_{2}, p_{3}, p_{4}\right)$ representa el conjunto de números cuánticos necesarios para determinar los autoestados, cuyos autovalores están dados por $\epsilon_{\bar{p}}=-\left(2 k|q B|+p_{3}^{2}+p_{4}^{2}\right)$. Cabe destacar que de estos cuatro números cuánticos, únicamente $k$ es un parámetro discreto. Trabajando en el espacio Euclídeo y eligiendo la representación de Weyl para las matrices de Dirac,

$$
\vec{\gamma}=\left(\begin{array}{cc}
0 & \vec{\sigma} \\
-\vec{\sigma} & 0
\end{array}\right), \quad \gamma_{4}=i \gamma_{0}=i\left(\begin{array}{ll}
0 & \mathcal{I} \\
\mathcal{I} & 0
\end{array}\right),
$$

tenemos

$$
\mathbb{E}_{\bar{p}}(x)=\sum_{\lambda= \pm} E_{\bar{p} \lambda}(x) \Delta^{\lambda}
$$

donde $\Delta^{+}=\operatorname{diag}(1,0,1,0), \Delta^{-}=\operatorname{diag}(0,1,0,1), \mathrm{y}$

$$
E_{\bar{p} \lambda}=N_{k_{\lambda}} e^{i\left(p_{2} x_{2}+p_{3} x_{3}+p_{4} x_{4}\right)} D_{k_{\lambda}}(\rho)
$$

donde $\rho=s \sqrt{2 /|q B|}\left(q B x_{1}-p_{2}\right)$, con $s=\operatorname{signo}(q B)$. El índice $k_{\lambda}$ está relacionado con el número cuántico $k$ por

$$
k_{ \pm}=k-\frac{1}{2} \pm \frac{s}{2},
$$


mientras que $N_{n}=(4 \pi|q B|)^{1 / 4} / \sqrt{n !}$. En la ecuación (C4) hemos introducido las funciones parabólicas cilíndricas definidas por

$$
D_{n}(x)=2^{-n / 2} e^{-x^{2} / 4} H_{n}(x / \sqrt{2}),
$$

donde $H_{n}(x)$ son los polinomios de Hermite, con la convención estándar $H_{-1}(x)=0$. Debe distinguirse el caso $k=0$, para el cual las autofunciones de Ritus $\mathbb{E}_{\bar{p}}(x)$ deben ser definidas como matrices de $2 \times 2$

$$
\mathbb{E}_{\left(0, p_{2}, p_{3}, p_{4}\right)}=(4 \pi|q B|)^{1 / 4} e^{i\left(p_{2} x_{2}+p_{3} x_{3}+p_{4} x_{4}\right)} e^{-\rho^{2} / 4} \mathbb{1}_{(2 \times 2)}
$$

donde $\mathbb{1}_{(2 \times 2)}$ es la matriz identidad en el subespacio donde $E_{\bar{p} \lambda}(x)$ es distinto de cero. Por otro lado, es fácil observar que las matrices $\Delta^{\lambda}$ satisfacen

$$
\Delta^{ \pm} \Delta^{ \pm}=\Delta^{ \pm}, \quad \Delta^{ \pm} \Delta^{\mp}=0, \quad \Delta^{ \pm} \gamma_{\perp}=\gamma_{\perp} \Delta^{\mp}, \quad \Delta^{ \pm} \gamma_{\|}=\gamma_{\|} \Delta^{ \pm},
$$

donde $\gamma_{\perp}=\left(\gamma_{1}, \gamma_{2}\right)$ y $\gamma_{\|}=\left(\gamma_{3}, \gamma_{4}\right)$.

Como es de esperarse, en la dirección del campo magnético la función $\mathbb{E}_{\bar{p}}(x)$ preserva la forma de la autofunción de energía de una partícula libre, estando etiquetada por un índice continuo $p_{3}$ que corresponde a la componente del momento paralela a $\vec{B}$. Ésta es también la situación en la dirección del tiempo imaginario. Por otra parte, los números cuánticos correspondientes al plano $x_{1} x_{2}$ dependen del gauge utilizado para describir el potencial vector $\mathcal{A}_{\mu}$. Aquí hemos elegido el gauge de Landau, para el cual los estados asociados con la dirección $x_{1}$ están cuantizados y etiquetados por el índice discreto $k$. En la dirección $x_{2}$ las autofunciones tienen la forma correspondiente a una partícula libre, con la particularidad de que los autovalores no dependen de $p_{2}$, por lo tanto los estados se encuentran degenerados. Esta última propiedad nos lleva a la útil relación

$$
\int \frac{d p_{2}}{2 \pi} \mathbb{E}_{\bar{p}}(x) \overline{\mathbb{E}}_{\bar{p}}(x)=\int \frac{d p_{2}}{2 \pi} \overline{\mathbb{E}}_{\bar{p}}(x) \mathbb{E}_{\bar{p}}(x)=|q B| P_{k, s},
$$

donde hemos definido $\overline{\mathbb{E}}_{\bar{p}}=\gamma_{0} \mathbb{E}_{\bar{p}}^{\dagger} \gamma_{0}$ y $P_{k, \pm 1}=\left(1-\delta_{k 0}\right) \mathcal{I}+\delta_{k 0} \Delta^{ \pm}$. Los operadores $P_{k, \pm 1}$ son proyectores, esto es, satisfacen $P_{k, s}=\left(P_{k, s}\right)^{2}$. También se puede ver que $P_{k, s} \mathbb{E}_{\bar{p}}=$ $\mathbb{E}_{\bar{p}} P_{k, s}=\mathbb{E}_{\bar{p}}$.

Las funciones de Ritus $\mathbb{E}_{\bar{p}}(x)$ satisfacen relaciones de ortonormalidad y completitud dadas por

$$
\begin{gathered}
\int d^{4} x \overline{\mathbb{E}}_{\bar{p}}(x) \mathbb{E}_{\bar{p}^{\prime}}(x)=\hat{\delta}_{\bar{p}, \bar{p}^{\prime}} P_{k, s}, \\
\oint_{\bar{p}} \mathbb{E}_{\bar{p}}(x) \overline{\mathbb{E}}_{\bar{p}}\left(x^{\prime}\right)=\delta^{(4)}\left(x-x^{\prime}\right),
\end{gathered}
$$


donde las siguientes notaciones fueron introducidas

$$
\sum_{p} \equiv \frac{1}{2 \pi} \sum_{k=0}^{\infty} \int \frac{d p_{2}}{2 \pi} \frac{d p_{3}}{2 \pi} \frac{d p_{4}}{2 \pi}, \quad \hat{\delta}_{\bar{p}^{,}, \bar{p}^{\prime}} \equiv(2 \pi)^{4} \delta_{k k^{\prime}} \delta\left(p_{2}-p_{2}^{\prime}\right) \delta\left(p_{3}-p_{3}^{\prime}\right) \delta\left(p_{4}-p_{4}^{\prime}\right) .
$$

Además, se verifica la importante identidad

$$
\Pi \mathbb{E}_{\bar{p}}(x)=\mathbb{E}_{\bar{p}}(x)\left(-s \sqrt{2 k|q B|} \gamma_{2}+p_{\|} \cdot \gamma_{\|}\right)
$$

donde $p_{\|}=\left(p_{3}, p_{4}\right)$.

Dadas las funciones de Ritus se puede definir la transformación de Ritus de alguna función de Dirac arbitraria $\psi(x)$ como

$$
\psi(x)=\sum_{\bar{p}} \mathbb{E}_{\bar{p}}(x) \psi_{\bar{p}}, \quad \bar{\psi}(x)=\sum_{\bar{p}} \bar{\psi}_{\bar{p}} \overline{\mathbb{E}}_{\bar{p}}(x) ，
$$

junto con la transformación inversa

$$
\psi_{\bar{p}}=\int d^{4} x \overline{\mathbb{E}}_{\bar{p}}(x) \psi(x), \quad \bar{\psi}_{\bar{p}}=\int d^{4} x \bar{\psi}(x) \mathbb{E}_{\bar{p}}(x) .
$$

Del mismo modo, la transformada de Ritus $\mathcal{O}_{\bar{p}, \bar{p}}$ de un operador arbitrario $\mathcal{O}_{x, x^{\prime}}$ se define como

$$
\begin{aligned}
\mathcal{O}_{\bar{p}, \bar{p}^{\prime}} & =\int d^{4} x d^{4} x^{\prime} \overline{\mathbb{E}}_{\bar{p}}(x) \mathcal{O}_{x, x^{\prime}} \mathbb{E}_{\bar{p}^{\prime}}\left(x^{\prime}\right), \\
\mathcal{O}_{x, x^{\prime}} & =\sum_{\bar{p}, \bar{p}^{\prime}} \mathbb{E}_{\bar{p}}(x) \mathcal{O}_{\bar{p}, \bar{p}^{\prime}} \overline{\mathbb{E}}_{\bar{p}^{\prime}}\left(x^{\prime}\right) .
\end{aligned}
$$





\section{Apéndice D}

\section{Cálculo de $G_{\bar{p}, \bar{p}^{\prime}}^{\lambda, f}$}

De acuerdo con la relación en la ecuación (5.11) se tiene

$$
G_{\bar{p}, \bar{p}^{\prime}}^{\lambda, f}=\int d^{4} x d^{4} x^{\prime} E_{\bar{p} \lambda}^{*}(x) \mathcal{G}\left(x-x^{\prime}\right) \exp \left[i \Phi_{f}\left(x, x^{\prime}\right)\right] E_{\bar{p}^{\prime} \lambda}\left(x^{\prime}\right),
$$

donde $\Phi_{f}\left(x, x^{\prime}\right)=\left(q_{f} B / 2\right)\left(x_{2}-x_{2}^{\prime}\right)\left(x_{1}+x_{1}^{\prime}\right)$, y las funciones $E_{\bar{p} \lambda}(x)$ están dadas en la ecuación (C4). Para trabajar con esta expresión introducimos la transformada de Fourier $\operatorname{de} \mathcal{G}(x)$,

$$
g\left(t^{2}\right)=\int d^{4} x e^{-i t \cdot x} \mathcal{G}(x)
$$

y realizamos el cambio de variables $x=z+y / 2, x^{\prime}=z-y / 2$. De este modo obtenemos

$$
G_{\bar{p}, \bar{p}^{\prime}}^{\lambda, f}=\int \frac{d^{4} t}{(2 \pi)^{4}} g\left(t^{2}\right) \int d^{4} y d^{4} z E_{p \lambda}^{*}\left(z+\frac{y}{2}\right) \exp (i t \cdot y) \exp \left(i q_{f} B y_{2} z_{1}\right) E_{p^{\prime} \lambda}\left(z-\frac{y}{2}\right) .
$$

Dadas las formas explícitas de las funciones $E_{\bar{p} \lambda}(x)$, las integrales sobre $y_{3}, y_{4}$ y $z_{2}, z_{3}, z_{4}$ pueden realizarse fácilmente. Obtenemos

$$
G_{\bar{p}, \bar{p}^{\prime}}^{\lambda, f}=(2 \pi)^{3} \delta\left(p_{2}-p_{2}^{\prime}\right) \delta\left(p_{3}-p_{3}^{\prime}\right) \delta\left(p_{4}-p_{4}^{\prime}\right) \Gamma_{k, k^{\prime}, p_{\|}}^{\lambda, f}
$$

donde

$$
\begin{aligned}
\Gamma_{k, k^{\prime}, p_{\|}}^{\lambda, f}= & N_{k_{\lambda}} N_{k_{\lambda}^{\prime}} \int \frac{d^{2} t_{\perp}}{(2 \pi)^{2}} g\left(t_{\perp}^{2}+p_{\|}^{2}\right) \int d z_{1} d^{2} y_{\perp} \\
& \exp \left(-i p_{2} y_{2}\right) \exp \left(i t_{\perp} \cdot y_{\perp}\right) \exp \left(i q_{f} B y_{2} z_{1}\right) D_{k_{\lambda}}(\rho) D_{k_{\lambda}^{\prime}}\left(\rho^{\prime}\right),
\end{aligned}
$$

$\operatorname{con} t_{\perp}=\left(t_{1}, t_{2}\right) \mathrm{y}$

$$
\rho=s_{f} \sqrt{\frac{2}{\left|q_{f} B\right|}}\left[q_{f} B\left(z_{1}+y_{1} / 2\right)-p_{2}\right], \quad \rho^{\prime}=s_{f} \sqrt{\frac{2}{\left|q_{f} B\right|}}\left[q_{f} B\left(z_{1}-y_{1} / 2\right)-p_{2}\right] .
$$


Recordemos que $s_{f}=\operatorname{signo}\left(q_{f} B\right)$, mientras que $k_{\lambda}$ está relacionada con $k$ según la ecuación (C5). Notemos ahora que la integración sobre $y_{2}$ introduce un factor $2 \pi \delta\left(q_{f} B z_{1}-p_{2}+\right.$ $\left.t_{2}\right)$, el cual permite realizar fácilmente la integral sobre $t_{2}$. Teniendo en cuenta la forma explícita de $\rho$ y $\rho^{\prime}$ obtenemos

$$
\begin{aligned}
\Gamma_{k, k^{\prime}, p_{\|}}^{\lambda, f}= & \frac{1}{\left[2 \pi 2^{k_{\lambda}+k_{\lambda}^{\prime}} k_{\lambda} ! k_{\lambda}^{\prime} !\right]^{1 / 2}} \int d \gamma d \eta d \psi g\left[\frac{\left|q_{f} B\right|}{2}\left(\gamma^{2}+\eta^{2}\right)+p_{\|}^{2}\right] \times \\
& \exp (i \gamma \psi) \exp \left(-\frac{\eta^{2}+\psi^{2}}{2}\right) H_{k_{\lambda}}\left(\frac{\eta+\psi}{\sqrt{2}}\right) H_{k_{\lambda}^{\prime}}\left(\frac{\eta-\psi}{\sqrt{2}}\right)
\end{aligned}
$$

donde hemos utilizado la expresión de $D_{n}$ en términos de los polinomios de Hermite, ecuación (C6), y por conveniencia hemos introducido las variables adimensionales

$$
\gamma=\sqrt{\frac{2}{\left|q_{f} B\right|}} t_{1}, \quad \eta=s_{f} \sqrt{\frac{2}{\left|q_{f} B\right|}}\left(q_{f} B z_{1}+p_{2}\right), \quad \psi=\sqrt{\frac{\left|q_{f} B\right|}{2}} y_{1} .
$$

Realizando un nuevo cambio de variables a coordenadas polares $r, \phi$ en el plano $\gamma \eta$, obtenemos

$$
\Gamma_{k, k^{\prime}, p_{\|}}^{\lambda, f}=\int_{0}^{\infty} d r r g\left(\frac{\left|q_{f} B\right|}{2} r^{2}+p_{\|}^{2}\right) \exp \left(-\frac{r^{2}}{2}\right) I_{k, k^{\prime}}^{\lambda}(r)
$$

donde

$$
\begin{aligned}
I_{k, k^{\prime}}^{\lambda}(r)=\frac{1}{\left[2 \pi 2^{k_{\lambda}+k_{\lambda}^{\prime}} k_{\lambda} ! k_{\lambda}^{\prime} !\right]^{1 / 2}} & \int_{0}^{2 \pi} d \phi \int_{-\infty}^{\infty} d \psi \exp \left[-\frac{(\psi-i r \cos \phi)^{2}}{2}\right] \times \\
& H_{k_{\lambda}}\left(\frac{r \sin \phi+\psi}{\sqrt{2}}\right) H_{k_{\lambda}^{\prime}}\left(\frac{r \sin \phi-\psi}{\sqrt{2}}\right) .
\end{aligned}
$$

Luego realizamos una traslación en el plano complejo $\psi, \psi \rightarrow \psi^{\prime}=\psi-i r \cos \phi$. Dado que el integrando en (D10) en una función analítica, utilizando el teorema de Cauchy se puede mostrar que la integral resultante puede realizarse sobre el eje $\operatorname{Im} \psi^{\prime}=0$. De este modo, obtenemos

$$
\begin{aligned}
& I_{k, k^{\prime}}^{\lambda}(r)=\frac{1}{\left[2 \pi 2^{k_{\lambda}+k_{\lambda}^{\prime}} k_{\lambda} ! k_{\lambda}^{\prime} !\right]^{1 / 2}} \int_{0}^{2 \pi} d \phi \int_{-\infty}^{\infty} d \psi \exp \left(-\frac{\psi^{2}}{2}\right) \\
& H_{k_{\lambda}}\left[\frac{i r \exp (-i \phi)+\psi}{\sqrt{2}}\right] H_{k_{\lambda}^{\prime}}\left[\frac{-i r \exp (i \phi)-\psi}{\sqrt{2}}\right] .
\end{aligned}
$$

Luego, utilizamos la relación $H_{n}(-x)=(-1)^{n} H_{n}(x)$ y la identidad [120]

$$
\int_{-\infty}^{\infty} d x e^{-x^{2}} H_{m}(x+y) H_{n}(x+z)=2^{n} \sqrt{\pi} m ! z^{n-m} L_{m}^{n-m}(-2 y z), \quad n \geqslant m
$$

donde $L_{b}^{a}(x)$ son los polinomios de Laguerre generalizados. Finalmente, usando

$$
\int_{0}^{2 \pi} d \phi \exp (i \phi m)=2 \pi \delta_{m 0}
$$


obtenemos

$$
I_{k, k^{\prime}}^{\lambda}(r)=2 \pi(-1)^{k_{\lambda}} L_{k_{\lambda}}\left(r^{2}\right) \delta_{k k^{\prime}} .
$$

Reemplazando la ecuación (D14) en (D9), y teniendo en cuenta la ecuación (D4), luego de un nuevo cambio de variables $r \rightarrow\left|p_{\perp}\right|=r \sqrt{\left|q_{f} B\right| / 2}$ llegamos a

$$
G_{\bar{p}, \bar{p}^{\prime}}^{\lambda, f}=\hat{\delta}_{p, p^{\prime}} g_{k, p, f}^{\lambda, f},
$$

donde $g_{k, p_{\|}}^{\lambda, f}$ está dada por la ecuación (5.12). 



\section{Apéndice E}

\section{Cálculo del propagador de quark a campo medio}

En esta sección describiremos la derivación de los propagadores de quarks $u$ y $d$ dentro de la aproximación de campo medio. En el espacio de Ritus éstos vienen dados por

$$
S_{\bar{p}, \bar{p}^{\prime}}^{\mathrm{MFA}, f}=\left(\mathcal{D}_{\bar{p}, \bar{p}^{\prime}}^{\mathrm{MFA}, f}\right)^{-1}=\hat{\delta}_{\bar{p}, \bar{p}^{\prime}}\left(\mathcal{D}_{k, p_{\|}}^{f}\right)^{-1}
$$

donde $\mathcal{D}_{k, p_{\|}}^{f}$, dado en (5.14), es diagonal en los índices de Landau/momento $\bar{p}$. Como este operador es no diagonal únicamente en el espacio de Dirac, su inversa puede calcularse fácilmente. Definiendo $\mathcal{S}_{k, p_{\|}}^{f}=\left(\mathcal{D}_{k, p_{\|}}^{f}\right)^{-1}$, se encuentra que $\mathcal{S}_{k, p_{\|}}^{f}$ puede escribirse como

$$
\mathcal{S}_{k, p_{\|}}^{f}=\sum_{\lambda= \pm}\left[\hat{A}_{k, p_{\|}}^{\lambda, f}-\hat{B}_{k, p_{\|}}^{\lambda, f} p_{\|} \cdot \gamma_{\|}+s_{f} \sqrt{2 k B_{f}}\left(\hat{C}_{k, p_{\|}}^{\lambda, f}-\hat{D}_{k, p_{\|}}^{\lambda, f} p_{\|} \cdot \gamma_{\|}\right) \gamma_{2}\right] \Delta^{\lambda}
$$

donde hemos definido $B_{f}=\left|q_{f} B\right|$, y las funciones $\hat{A}_{k, p_{\|}}^{\lambda, f}$ a $\hat{D}_{k, p_{\|}}^{\lambda, f}$ están dadas en las ecuaciones (5.23-5.26). Hay que notar que en el caso particular de $k=0$ (es decir $k_{\lambda}=0$ ó -1) el espacio de Dirac se ve reducido a uno de dos dimensiones, por lo tanto en este caso sólo es necesario considerar los coeficientes $\hat{A}_{k, p_{\|}}^{\lambda, f}$ y $\hat{B}_{k, p_{\|}}^{\lambda, f}$.

Para encontrar la expresión de los propagadores en el espacio de coordenadas se debe calcular la antitransformada de Ritus de $S_{\bar{p}, \bar{p}^{\prime}}^{\mathrm{MFA},}$. Es decir

$$
\begin{aligned}
& S_{x, x^{\prime}}^{\mathrm{MFA}, f}= \sum_{\bar{p}^{\prime}, \bar{p}^{\prime}} \mathbb{E}_{\bar{p}}(x) S_{\bar{p}, \bar{p}^{\prime}}^{\mathrm{MFA}, f} \overline{\mathbb{E}}_{\bar{p}^{\prime}}\left(x^{\prime}\right) \\
&=\frac{1}{2 \pi} \sum_{k=0}^{\infty} \int \frac{d^{2} p_{\|}}{(2 \pi)^{2}} e^{i p_{\|} \cdot \Delta x_{\|}} \sum_{\lambda, \lambda^{\prime}= \pm} I^{\lambda \lambda^{\prime}}\left[\delta_{\lambda \lambda^{\prime}}\left(\hat{A}_{k, p_{\|}}^{\lambda, f}-\hat{B}_{k, p_{\|}}^{\lambda, f} p_{\|} \cdot \gamma_{\|}\right) \Delta^{\lambda}\right. \\
&\left.\quad+s_{f} \sqrt{2 k B_{f}}\left(1-\delta_{\lambda \lambda^{\prime}}\right)\left(\hat{C}_{k, p_{\|}}^{\lambda^{\prime}, f}-\hat{D}_{k, p_{\|}}^{\lambda^{\prime}, f} p_{\|} \cdot \gamma_{\|}\right) \gamma_{2} \Delta^{\lambda^{\prime}}\right]
\end{aligned}
$$


donde hemos definido $\Delta x_{\|}=\left(\Delta x_{3}, \Delta x_{4}\right)$, con $\Delta x_{i}=x_{i}-x_{i}^{\prime}$, y las integrales $I^{\lambda \lambda^{\prime}}$ están dadas por

$$
I^{\lambda \lambda^{\prime}}=N_{k_{\lambda}} N_{k_{\lambda^{\prime}}} \int \frac{d p_{2}}{2 \pi} e^{i p_{2}\left(x_{2}-x_{2}^{\prime}\right)} D_{k_{\lambda}}(\rho) D_{k_{\lambda^{\prime}}}\left(\rho^{\prime}\right),
$$

$\operatorname{con} \rho^{(\prime)}=s_{f} \sqrt{2 / B_{f}}\left[B_{f} x_{1}^{(\prime)}-p_{2}\right]=\sqrt{2 B_{f}}\left[x_{1}^{(\prime)}-\left(s_{f} / B_{f}\right) p_{2}\right]$. Analizaremos las integrales $I^{ \pm \pm}$y $I^{ \pm \mp}$ separadamente. Considerando las expresiones explícitas para $N_{k_{\lambda}}$ y $D_{k_{\lambda}}(x)$ [ver ecuación (C6)], y realizando la traslación $p_{2}=q_{2}+s_{f} B_{f}\left(x_{1}+x_{1}^{\prime}\right) / 2$, se obtiene

$$
\begin{aligned}
I^{\lambda \lambda=} & \sqrt{\frac{B_{f}}{\pi}} \frac{2^{-k_{\lambda}}}{k !} \exp \left[i \Phi_{f}\left(x, x^{\prime}\right)\right] \exp \left(-\frac{B_{f} \Delta x_{1}^{2}}{4}\right) \int_{-\infty}^{\infty} d q_{2} \exp \left(i q_{2} \Delta x_{2}\right) \times \\
& \exp \left(-\frac{q_{2}^{2}}{B_{f}}\right) H_{k_{\lambda}}\left(\frac{\sqrt{B_{f}} \Delta x_{1}}{2}-\frac{s_{f} q_{2}}{\sqrt{B_{f}}}\right) H_{k_{\lambda}}\left(-\frac{\sqrt{B_{f}} \Delta x_{1}}{2}-\frac{s_{f} q_{2}}{\sqrt{B_{f}}}\right)
\end{aligned}
$$

donde $\Phi_{f}\left(x, x^{\prime}\right)$ es la fase de Schwinger que ya hemos definido. Ahora es posible realizar una traslación en el campo complejo a una nueva variable $\omega=\left(q_{2}-i B_{f} \Delta x_{2} / 2\right) s_{f} / \sqrt{B_{f}}$. Como el integrando es una función analítica de $q_{2}$ en todo el plano, la integral puede calcularse sobre el eje $\operatorname{Im} \omega=0$. Se obtiene de este modo

$$
\begin{aligned}
I^{\lambda \lambda}= & \frac{B_{f}}{\sqrt{\pi}} \frac{2^{-k_{\lambda}}}{k !} \exp \left[i \Phi_{f}\left(x, x^{\prime}\right)\right] \exp \left(-\frac{B_{f} \Delta x_{\perp}^{2}}{4}\right) \int_{-\infty}^{\infty} d \omega \exp \left(-\omega^{2}\right) \times \\
& H_{k_{\lambda}}\left[\omega-\frac{\sqrt{B_{f}}}{2}\left(\Delta x_{1}-i s_{f} \Delta x_{2}\right)\right] H_{k_{\lambda}}\left[\omega+\frac{\sqrt{B_{f}}}{2}\left(\Delta x_{1}+i s_{f} \Delta x_{2}\right)\right]
\end{aligned}
$$

donde $\Delta x_{\perp}=\left(\Delta x_{1}, \Delta x_{2}\right)$. La integral en (E6) puede evaluarse usando la relación en (D12), lo cual conduce a

$$
I^{\lambda \lambda}=B_{f} \exp \left[i \Phi_{f}\left(x, x^{\prime}\right)\right] \exp \left[-\frac{B_{f} \Delta x_{\perp}^{2}}{4}\right] L_{k_{\lambda}}\left(\frac{B_{f} \Delta x_{\perp}^{2}}{2}\right) .
$$

Considerando la integral

$$
K^{(0)}\left(m, y_{\perp}\right)=\int d^{2} p_{\perp} e^{i p_{\perp} \cdot y_{\perp}} \exp \left(-\frac{p_{\perp}^{2}}{B_{f}}\right) L_{m}\left(\frac{2 p_{\perp}^{2}}{B_{f}}\right),
$$

donde $p_{\perp}=\left(p_{1}, p_{2}\right), y_{\perp}=\left(y_{1}, y_{2}\right)$, se obtiene

$$
\begin{aligned}
K^{(0)}\left(m, y_{\perp}\right) & =\int_{0}^{\infty} d\left|p_{\perp}\right|\left|p_{\perp}\right| \exp \left(-\frac{p_{\perp}^{2}}{B_{f}}\right) L_{m}\left(\frac{2 p_{\perp}^{2}}{B_{f}}\right) \int_{0}^{2 \pi} d \theta e^{i\left|p_{\perp}\right|\left(y_{1} \cos \theta+y_{2} \sin \theta\right)} \\
& =2 \pi \int_{0}^{\infty} d\left|p_{\perp}\right|\left|p_{\perp}\right| \exp \left(-\frac{p_{\perp}^{2}}{B_{f}}\right) L_{m}\left(\frac{2 p_{\perp}^{2}}{B_{f}}\right) J_{0}\left(\left|p_{\perp}\right|\left|y_{\perp}\right|\right) \\
& =\pi B_{f}(-1)^{m} \exp \left(-\frac{B_{f} y_{\perp}^{2}}{4}\right) L_{m}\left(\frac{B_{f} y_{\perp}^{2}}{2}\right)
\end{aligned}
$$


donde $J_{0}(x)$ es una función de Bessel. La última igualdad en la ecuación (E9) se obtuvo usando la siguiente relación general, la cual involucra polinomios de Laguerre generalizados y funciones de Bessel:

$$
\int_{0}^{\infty} d x x^{v+1} e^{-\beta x^{2}} L_{m}^{v}\left(\alpha x^{2}\right) J_{v}(x y)=(2 \beta)^{-v-1}\left(1-\frac{\alpha}{\beta}\right)^{m} y^{v} e^{-y^{2} /(4 \beta)} L_{m}^{v}\left[\frac{\alpha y^{2}}{4 \beta(\alpha-\beta)}\right] .
$$

De las ecuaciones (E7), (E8) y (E9) finalizamos con

$$
\begin{aligned}
I^{\lambda \lambda} & =\frac{1}{\pi} \exp \left[i \Phi_{f}\left(x, x^{\prime}\right)\right](-1)^{k_{\lambda}} K^{(0)}\left(k_{\lambda}, \Delta x_{\perp}\right) \\
& =4 \pi \exp \left[i \Phi_{f}\left(x, x^{\prime}\right)\right](-1)^{k_{\lambda}} \int \frac{d^{2} p_{\perp}}{(2 \pi)^{2}} e^{i p_{\perp} \cdot \Delta x_{\perp}} \exp \left(-\frac{p_{\perp}^{2}}{B_{f}}\right) L_{k_{\lambda}}\left(\frac{2 p_{\perp}^{2}}{B_{f}}\right) .
\end{aligned}
$$

Un procedimiento similar se lleva a cabo para el cálculo de las integrales $I^{ \pm \mp}$. Realizando los mismos cambios de variables que en el caso anterior obtenemos

$$
\begin{aligned}
I^{ \pm \mp}= & \frac{B_{f}}{\sqrt{\pi}} \frac{2^{-\left(k_{+}+k_{-}\right) / 2}}{\sqrt{k}(k-1) !} \exp \left[i \Phi_{f}\left(x, x^{\prime}\right)\right] \exp \left(-\frac{B_{f} \Delta x_{\perp}^{2}}{4}\right)(-1)^{k_{+}+k_{-}} \int_{-\infty}^{\infty} d \omega e^{-\omega^{2}} \times \\
& H_{k_{+}}\left[\omega \mp \frac{\sqrt{B_{f}}}{2}\left(\Delta x_{1} \mp i s_{f} \Delta x_{2}\right)\right] H_{k_{-}}\left[\omega \pm \frac{\sqrt{B_{f}}}{2}\left(\Delta x_{1} \pm i s_{f} \Delta x_{2}\right)\right] \\
= & B_{f} \sqrt{\frac{B_{f}}{2 k}} s_{f} \exp \left[i \Phi_{f}\left(x, x^{\prime}\right)\right]\left( \pm \Delta x_{1}-i \Delta x_{2}\right) \exp \left(-\frac{B_{f} \Delta x_{\perp}^{2}}{4}\right) L_{k-1}^{1}\left(\frac{B_{f} \Delta x_{\perp}^{2}}{2}\right),
\end{aligned}
$$

donde hemos usado nuevamente la relación en la ecuación (D12) para evaluar la integral sobre $\omega$. Notar que para $k=0$ se tiene $I^{+-}=I^{-+}=0$ automáticamente a partir de la definición en (E4), ya que $k_{+}=-1$ o $k_{-}=-1$, y $D_{-1}\left(\rho^{(\prime)}\right)=0$. Ahora consideremos las integrales

$$
K_{j}^{(1)}\left(m, y_{\perp}\right)=\int d^{2} p_{\perp} p_{j} e^{i p_{\perp} \cdot y_{\perp}} \exp \left(-\frac{p_{\perp}^{2}}{B_{f}}\right) L_{m}^{1}\left(\frac{2 p_{\perp}^{2}}{B_{f}}\right)
$$

donde $j=1$,2. Usando la ecuación (E10) con $v=1$ es sencillo demostrar que

$$
\begin{aligned}
K_{j}^{(1)}\left(m, y_{\perp}\right) & =2 \pi i \frac{y_{j}}{\left|y_{\perp}\right|} \int_{0}^{\infty} d\left|p_{\perp}\right| p_{\perp}^{2} \exp \left(-\frac{p_{\perp}^{2}}{B_{f}}\right) L_{m}^{1}\left(\frac{2 p_{\perp}^{2}}{B_{f}}\right) J_{1}\left(\left|p_{\perp}\right|\left|y_{\perp}\right|\right) \\
& =\frac{\pi}{2} i B_{f}^{2}(-1)^{m} y_{j} \exp \left(-\frac{B_{f} y_{\perp}^{2}}{4}\right) L_{m}^{1}\left(\frac{B_{f} y_{\perp}^{2}}{2}\right)
\end{aligned}
$$

de donde obtenemos

$$
\begin{aligned}
& I^{ \pm \mp}=(-i) \frac{2}{\pi} s_{f} \exp \left[i \Phi_{f}\left(x, x^{\prime}\right)\right] \frac{(-1)^{k}}{\sqrt{2 k B_{f}}}\left[\mp K_{1}^{(1)}\left(k-1, \Delta x_{\perp}\right)+i K_{2}^{(1)}\left(k-1, \Delta x_{\perp}\right)\right] \\
&=-i 8 \pi s_{f} \exp \left[i \Phi_{f}\left(x, x^{\prime}\right)\right] \frac{(-1)^{k}}{\sqrt{2 k B_{f}}} \int \frac{d^{2} p_{\perp}}{(2 \pi)^{2}} e^{i \Delta x_{\perp} \cdot p_{\perp}} \times \\
&\left(\mp p_{1}+i p_{2}\right) \exp \left(-\frac{p_{\perp}^{2}}{B_{f}}\right) L_{k-1}^{1}\left(\frac{2 p_{\perp}^{2}}{B_{f}}\right) .
\end{aligned}
$$


Los resultados en (E11) y (E15) pueden combinarse de la siguiente forma

$$
\begin{aligned}
I^{\lambda \lambda^{\prime}}= & 4 \pi(-i)^{k_{\lambda}+k_{\lambda^{\prime}}}\left(\frac{2}{k B_{f}}\right)^{\left|k_{\lambda}-k_{\lambda^{\prime}}\right|} \exp \left[i \Phi_{f}\left(x, x^{\prime}\right)\right] \int \frac{d^{2} p_{\perp}}{(2 \pi)^{2}} e^{i \Delta x_{\perp} \cdot p_{\perp}} \exp \left(-\frac{p_{\perp}^{2}}{B_{f}}\right) \times \\
& {\left.\left[\left(k_{\lambda}-k_{\lambda^{\prime}}\right) p_{1}-i s_{f} p_{2}\right]^{\left|k_{\lambda}-k_{\lambda^{\prime}}\right|} L_{\left(k_{\lambda}+k_{\lambda^{\prime}}-\left|k_{\lambda}-k_{\lambda^{\prime}}\right|\right) / 2}^{\left|k_{\lambda}-k_{\lambda^{\prime}}\right|} \frac{2 p_{\perp}^{2}}{B_{f}}\right) }
\end{aligned}
$$

(una expresión análoga es obtenida en la Ref. [103]). Reemplazando en (E3), y notando que $-i\left( \pm p_{1}+i p_{2}\right) \gamma_{2} \Delta^{ \pm}=p_{\perp} \cdot \gamma_{\perp} \Delta^{ \pm}$, finalmente llegamos a

$$
S_{x, x^{\prime}}^{\mathrm{MFA}, f}=\exp \left[i \Phi_{f}\left(x, x^{\prime}\right)\right] \int \frac{d^{4} p}{(2 \pi)^{4}} e^{i p \cdot\left(x-x^{\prime}\right)} \tilde{S}^{f}\left(p_{\perp}, p_{\|}\right),
$$

donde $\tilde{S}^{f}\left(p_{\perp}, p_{\|}\right)$en el modelo NJL no local está dada por la expresión (5.22). 


\section{Apéndice $F$}

\section{Cálculo de la masa del pion en presencia de campo magnético}

Describiremos aquí cómo se deriva la relación (5.42). La ecuación (5.36) se puede reescribir en la forma

$$
F\left(0, k_{\|}^{2}\right)=-128 \pi^{2} N_{C} \sum_{f=u, d} \frac{1}{B_{f}^{2}} \sum_{k, k^{\prime}=0}^{\infty} \int_{q_{\|}}\left[\sum_{\lambda= \pm} F_{k k^{\prime}, q_{\|}^{+} q_{\|}^{-}}^{\lambda, f(A B)} I_{k k^{\prime}, q_{\|}}^{\lambda, f(0)}+F_{k k^{\prime}, q_{\|}^{+} q_{\|}^{-}}^{+, f(C D)} I_{k k^{\prime}, q_{\|}}^{f(1)}\right],
$$

donde

$$
\begin{aligned}
I_{k k^{\prime}, q_{\|}}^{\lambda, f(0)=} & (-1)^{k+k^{\prime}} \int_{q_{\perp} p_{\perp} p_{\perp}^{\prime}} \exp \left[\frac{i 2 \phi\left(q_{\perp}, p_{\perp}, p_{\perp}^{\prime}\right)}{B_{f}}\right] \exp \left[-\frac{p_{\perp}^{2}+p_{\perp}^{\prime 2}}{B_{f}}\right] \times \\
& g\left(q_{\perp}^{2}+q_{\|}^{2}\right) g\left[\left(p_{\perp}^{\prime}+p_{\perp}-q_{\perp}\right)^{2}+q_{\|}^{2}\right] L_{k_{\lambda}}\left(\frac{2 p_{\perp}^{2}}{B_{f}}\right) L_{k_{\lambda}^{\prime}}\left(\frac{2 p_{\perp}^{\prime 2}}{B_{f}}\right), \\
I_{k k^{\prime}, q_{\|}}^{f(1)}= & 8(-1)^{k+k^{\prime}} \int_{q_{\perp} p_{\perp} p_{\perp}^{\prime}} \exp \left[\frac{i 2 \phi\left(q_{\perp}, p_{\perp}, p_{\perp}^{\prime}\right)}{B_{f}}\right] \exp \left[-\frac{p_{\perp}^{2}+p_{\perp}^{\prime 2}}{B_{f}}\right] \times \\
& \left(p_{\perp} \cdot p_{\perp}^{\prime}\right) g\left(q_{\perp}^{2}+q_{\|}^{2}\right) g\left[\left(p_{\perp}^{\prime}+p_{\perp}-q_{\perp}\right)^{2}+q_{\|}^{2}\right] L_{k-1}^{1}\left(\frac{2 p_{\perp}^{2}}{B_{f}}\right) L_{k^{\prime}-1}^{1}\left(\frac{2 p_{\perp}^{\prime 2}}{B_{f}}\right) .
\end{aligned}
$$

Estas integrales pueden resolverse tomando las transformaciones de Laguerre-Fourier de los factores de forma no locales dadas en (5.41). Obtenemos de este modo 


$$
\begin{aligned}
I_{k k^{\prime}, q_{\|}}^{\lambda, f(0)=} & 4(-1)^{k+k^{\prime}} \sum_{m, m^{\prime}=0}^{\infty}(-1)^{m+m^{\prime}} g_{m, q_{\|}}^{\lambda, f} g_{m^{\prime}, q_{\|}}^{\lambda, f} \int_{q_{\perp} p_{\perp} p_{\perp}^{\prime}} \exp \left[\frac{i 2 \phi\left(q_{\perp}, p_{\perp}, p_{\perp}^{\prime}\right)}{B_{f}}\right] \times \\
& \exp \left[-\frac{p_{\perp}^{2}+p_{\perp}^{\prime 2}+q_{\perp}^{2}+\left(p_{\perp}^{\prime}+p_{\perp}-q_{\perp}\right)^{2}}{B_{f}}\right] \times \\
& L_{k_{\lambda}}\left(\frac{2 p_{\perp}^{2}}{B_{f}}\right) L_{k_{\lambda}^{\prime}}\left(\frac{2 p_{\perp}^{\prime 2}}{B_{f}}\right) L_{m_{\lambda}}\left(\frac{2 q_{\perp}^{2}}{B_{f}}\right) L_{m_{\lambda}^{\prime}}\left[\frac{2\left(p_{\perp}^{\prime}+p_{\perp}-q_{\perp}\right)^{2}}{B_{f}}\right], \\
I_{k k^{\prime}, q_{\|}}^{f(1)}= & 32(-1)^{k+k^{\prime}} \sum_{m, m^{\prime}=0}^{\infty}(-1)^{m+m^{\prime}} g_{m, q_{\|}}^{+, f} g_{m^{\prime}, q_{\|}}^{-, f} \int_{q_{\perp}} p_{\perp} p_{\perp}^{\prime} \exp \left[\frac{i 2 \phi\left(q_{\perp}, p_{\perp}, p_{\perp}^{\prime}\right)}{B_{f}}\right] \times \\
& \exp \left[-\frac{p_{\perp}^{2}+p_{\perp}^{\prime 2}+q_{\perp}^{2}+\left(p_{\perp}^{\prime}+p_{\perp}-q_{\perp}\right)^{2}}{B_{f}}\right]\left(p_{\perp} \cdot p_{\perp}^{\prime}\right) \times \\
& L_{k-1}^{1}\left(\frac{2 p_{\perp}^{2}}{B_{f}}\right) L_{k^{\prime}-1}^{1}\left(\frac{2 p_{\perp}^{\prime 2}}{B_{f}}\right) L_{m_{+}}\left(\frac{2 q_{\perp}^{2}}{B_{f}}\right) L_{m_{-}^{\prime}}\left[\frac{2\left(p_{\perp}^{\prime}+p_{\perp}-q_{\perp}\right)^{2}}{B_{f}}\right]
\end{aligned}
$$

Si cambiamos las variables de integración, definiendo los vectores adimensionales $u=$ $-\sqrt{\left(2 / B_{f}\right)} p_{\perp}, v=\sqrt{\left(2 / B_{f}\right)} p_{\perp}^{\prime}, w=\sqrt{\left(2 / B_{f}\right)}\left(p_{\perp}-q_{\perp}\right)$, las integrales resultan

$$
\begin{aligned}
I_{k k^{\prime}, q_{\|}}^{\lambda, f(0)} & =\frac{B_{f}^{3}}{2}(-1)^{k+k^{\prime}} \sum_{m, m^{\prime}=0}^{\infty}(-1)^{m+m^{\prime}} g_{m, q_{\|}}^{\lambda, f} g_{m m^{\prime}, q_{\|}}^{\lambda, f} K_{k k^{\prime} m m^{\prime}}^{\lambda, f(0)}, \\
I_{k k^{\prime}, q_{\|}}^{f(1)} & =2 B_{f}^{4}(-1)^{k+k^{\prime}} \sum_{m, m^{\prime}=0}^{\infty}(-1)^{m+m^{\prime}-1} g_{m, q_{\|}}^{+, f} g_{m^{\prime}, q_{\|}}^{-, f} K_{k k^{\prime} m m^{\prime}}^{f(1)},
\end{aligned}
$$

donde

$$
\begin{aligned}
K_{k k^{\prime} m m^{\prime}}^{\lambda, f(0)}= & \int_{u v w} \exp \left[-w^{2}\right] \exp \left[-u^{2}-u \cdot w-i s_{f}\left(u_{1} w_{2}-u_{2} w_{1}\right)\right] L_{k_{\lambda}}\left(u^{2}\right) L_{m_{\lambda}}\left[(u+w)^{2}\right] \times \\
K_{k k^{\prime} m m^{\prime}}^{f(1)}= & -\int_{u v w} \exp \left[-v^{2}-v \cdot w-i s_{f}\left(v_{1} w_{2}-v_{2} w_{1}\right)\right] L_{k_{\lambda}^{\prime}}\left(v^{2}\right) L_{m_{\lambda}^{\prime}}\left[(v+w)^{2}\right] \\
& (u \cdot v) \exp \left[-v^{2}-v \cdot w-i s_{f}\left(v_{1} w_{2}-v_{2} w_{1}\right)\right] L_{k^{\prime}-1}\left(v^{2}\right) L_{m_{-}^{\prime}}\left[(v+w)^{2}\right] .
\end{aligned}
$$

Se puede observar que $K_{k k^{\prime} m m^{\prime}}^{\lambda, f(0)}$ y $K_{k k^{\prime} m m^{\prime}}^{f(1)}$ no dependen del momento o en el campo magnético. Para calcular estas cantidades contamos con la ayuda de las siguientes relaciones útiles 


$$
\begin{gathered}
\frac{1}{2 \pi} \int_{0}^{2 \pi} d \theta L_{n}\left(x^{2}+y^{2}+2 x y \cos \theta\right) \exp [-x y \exp ( \pm i \theta)]=L_{n}\left(x^{2}\right) L_{n}\left(y^{2}\right), \\
\frac{1}{2 \pi} \int_{0}^{2 \pi} d \theta \cos \theta L_{n}\left(x^{2}+y^{2}+2 x y \cos \theta\right) \exp [-x y \exp ( \pm i \theta)] \\
=-\frac{x y}{2}\left[\frac{L_{n}^{1}\left(x^{2}\right) L_{n}^{1}\left(y^{2}\right)}{n+1}+\frac{L_{n-1}^{1}\left(x^{2}\right) L_{n-1}^{1}\left(y^{2}\right)}{n}\right], \\
\frac{1}{2 \pi} \int_{0}^{2 \pi} d \theta \sin \theta L_{n}\left(x^{2}+y^{2}+2 x y \cos \theta\right) \exp [-x y \exp ( \pm i \theta)] \\
=\mp \frac{i x y}{2}\left[\frac{L_{n}^{1}\left(x^{2}\right) L_{n}^{1}\left(y^{2}\right)}{n+1}-\frac{L_{n-1}^{1}\left(x^{2}\right) L_{n-1}^{1}\left(y^{2}\right)}{n}\right],
\end{gathered}
$$

junto con las propiedades de ortogonalidad de los polinomios de Laguerre generalizados. En el caso de $K_{k k^{\prime} m m^{\prime \prime}}^{\lambda, f(0)}$ a partir de la relación (F8) se obtiene

$$
\begin{aligned}
K_{k k^{\prime} m m^{\prime}}^{\lambda, f(0)=} & \frac{1}{(4 \pi)^{2}} \int_{w} \exp \left(-w^{2}\right) \int_{0}^{\infty} d u^{2} \exp \left(-u^{2}\right) L_{k_{\lambda}}\left(u^{2}\right) L_{m_{\lambda}}\left(u^{2}\right) L_{m_{\lambda}}\left(w^{2}\right) \times \\
& \int_{0}^{\infty} d v^{2} \exp \left(-v^{2}\right) L_{k_{\lambda}^{\prime}}\left(v^{2}\right) L_{m_{\lambda}^{\prime}}\left(v^{2}\right) L_{m_{\lambda}^{\prime}}\left(w^{2}\right) \\
= & \frac{1}{(4 \pi)^{3}} \delta_{k m} \delta_{k^{\prime} m^{\prime}} \delta_{m m^{\prime}}
\end{aligned}
$$

y como consecuencia

$$
I_{k k^{\prime}, q_{\|}}^{\lambda, f(0)}=\frac{B_{f}^{3}}{128 \pi^{3}} g_{k, q_{\|}}^{\lambda, f} g_{k, q_{\|}}^{\lambda, f} \delta_{k k^{\prime}}
$$

Finalmente, usando las ecuaciones (F9) y (F10) obtenemos

$$
K_{k k^{\prime} m m^{\prime}}^{f(1)}=-\frac{1}{128 \pi^{3}} k \delta_{k k^{\prime}}\left(\delta_{m+1 k_{-}} \delta_{m^{\prime} k_{+}}+\delta_{m k_{-}} \delta_{m^{\prime}-1 k_{+}}\right)
$$

lo cual conduce a

$$
I_{k k^{\prime}, q_{\|}}^{f(1)}=\frac{k B_{f}^{4}}{32 \pi^{3}} g_{k, q_{\|}}^{+, f} g_{k, q_{\|}}^{-, f} \delta_{k k^{\prime}}
$$

Reemplazando los resultados (F12) y (F14) en la ecuación (F1) llegamos a nuestra expresión final (5.42). 



\section{Apéndice G}

\section{Cálculo de la constante de decaimiento del pion en presencia de campo magnético}

Aquí se discutirá la derivación de nuestros resultados en las ecuaciones (5.66) y (5.68). Partiremos de las expresiones (5.62), e introducimos la transformación de Fourier-Laguerre de $g\left(q^{2}\right)$. Luego, cambiamos el orden de las integrales y las sumas obteniendo

$$
\begin{aligned}
\left.t_{\mu} F_{\mu}^{(\mathrm{II})}(t)\right|_{t_{\perp}=0}= & i 128 \pi^{2} N_{C} \sum_{f=u, d} \frac{1}{B_{f}^{2}} \sum_{k, k^{\prime}, m=0}^{\infty} \int_{q_{\|}}\left\{\sum_{\lambda= \pm} g_{m, q_{\|}}^{\lambda, f} \times\right. \\
& {\left[\left(t_{\|} \cdot q_{\|}^{-}\right) \hat{A}_{k, q_{\|}^{+}}^{\lambda, f} \hat{B}_{k^{\prime}, q_{\|}^{-}}^{\lambda, f}-\left(t_{\|} \cdot q_{\|}^{+}\right) \hat{A}_{k^{\prime}, q_{\|}^{-}}^{\lambda, f} \hat{B}_{k, q_{\|}^{+}}^{\lambda, f} \tilde{K}_{k k^{\prime} m}^{\lambda, f(0)}+\right.} \\
& \left.8 i g_{m, q_{\|}}^{+, f}\left[\left(t_{\|} \cdot q_{\|}^{-}\right) \hat{C}_{k, q_{\|}^{+}}^{+, f} \hat{D}_{k^{\prime}, q_{\|}^{-}}^{+, f}-\left(t_{\|} \cdot q_{\|}^{+}\right) \hat{C}_{k^{\prime}, q_{\|}^{-}}^{+, f} \hat{D}_{k, q_{\|}^{+}}^{+, f}\right] \tilde{K}_{k k^{\prime} m}^{f(1)}\right\},
\end{aligned}
$$

donde

$$
\begin{aligned}
\tilde{K}_{k k^{\prime} m}^{\lambda, f(0)=} & (-1)^{k+k^{\prime}+m_{\lambda}} \int_{q_{\perp} p_{\perp} p_{\perp}^{\prime}} \exp \left[-\frac{i 2 \phi\left(q_{\perp}, p_{\perp}, p_{\perp}^{\prime}\right)}{B_{f}}\right] \times \\
& \exp \left[-\frac{p_{\perp}^{2}+p_{\perp}^{\prime 2}+q_{\perp}^{2}}{B_{f}}\right] L_{k_{\lambda}}\left(\frac{2 p_{\perp}^{2}}{B_{f}}\right) L_{k_{\lambda}^{\prime}}\left(\frac{2 p_{\perp}^{\prime 2}}{B_{f}}\right) L_{m_{\lambda}}\left(\frac{2 q_{\perp}^{2}}{B_{f}}\right), \\
\tilde{K}_{k k^{\prime} m}^{f(1)}= & (-1)^{k+k^{\prime}+m_{+}} \int_{q_{\perp} p_{\perp} p_{\perp}^{\prime}} \exp \left[-\frac{i 2 \phi\left(q_{\perp}, p_{\perp}, p_{\perp}^{\prime}\right)}{B_{f}}\right]\left(p_{1} p_{2}^{\prime}-p_{2} p_{1}^{\prime}\right) \times \\
& \exp \left[-\frac{p_{\perp}^{2}+p_{\perp}^{\prime 2}+q_{\perp}^{2}}{B_{f}}\right] L_{k-1}^{1}\left(\frac{2 p_{\perp}^{2}}{B_{f}}\right) L_{k^{\prime}-1}^{1}\left(\frac{2 p_{\perp}^{\prime 2}}{B_{f}}\right) L_{m_{+}}\left(\frac{2 q_{\perp}^{2}}{B_{f}}\right) .
\end{aligned}
$$

Ahora realizamos un cambio de variables de integración, definiendo los vectores adimensionales $u=\sqrt{\left(2 / B_{f}\right)} q_{\perp}, v=\sqrt{\left(2 / B_{f}\right)} p_{\perp}, w=\sqrt{\left(2 / B_{f}\right)}\left(p_{\perp}^{\prime}-p_{\perp}\right)$. Las expresiones 
para $K_{k k^{\prime} m}^{\lambda, f(0)}$ y $K_{k k^{\prime} m}^{f(1)}$ toman la forma

$$
\begin{aligned}
\tilde{K}_{k k^{\prime} m}^{\lambda, f(0)}= & (-1)^{k+k^{\prime}+m_{\lambda}} \frac{B_{f}^{3}}{8} \int_{v w} \exp \left[i s_{f}\left(v_{1} w_{2}-v_{2} w_{1}\right)\right] \exp \left[-\left(v^{2}+v \cdot w+\frac{w^{2}}{2}\right)\right] \times \\
& L_{k_{\lambda}}\left(v^{2}\right) L_{k_{\lambda}^{\prime}}\left[(v+w)^{2}\right] \int_{u} \exp \left(-\frac{u^{2}}{2}\right) L_{m_{\lambda}}\left(u^{2}\right) \exp \left[i s_{f}\left(w_{1} u_{2}-w_{2} u_{1}\right)\right],(\mathrm{G} 4) \\
\tilde{K}_{k k^{\prime} m}^{f(1)}= & (-1)^{k+k^{\prime}+m_{+}} \frac{B_{f}^{4}}{16} \int_{v w} \exp \left[i s_{f}\left(v_{1} w_{2}-v_{2} w_{1}\right)\right] \exp \left[-\left(v^{2}+v \cdot w+\frac{w^{2}}{2}\right)\right] \times \\
& \left(v_{1} w_{2}-v_{2} w_{1}\right) L_{k-1}^{1}\left(v^{2}\right) L_{k^{\prime}-1}^{1}\left[(v+w)^{2}\right] \times \\
& \int_{u} \exp \left(-\frac{u^{2}}{2}\right) L_{m_{+}}\left(u^{2}\right) \exp \left[i s_{f}\left(w_{1} u_{2}-w_{2} u_{1}\right)\right] .
\end{aligned}
$$

Para evaluar las integrales en $u$, fijamos el vector externo $w$ sobre la dirección 1 , lo cual conduce a la expresión

$$
\begin{aligned}
\int_{u} \exp \left(-\frac{u^{2}}{2}\right) L_{m_{\lambda}}\left(u^{2}\right) \exp \left[i s_{f}\left(w_{1} u_{2}-w_{2} u_{1}\right)\right] \\
=\frac{1}{(2 \pi)^{2}} \int_{0}^{\infty} d|u||u| \exp \left(-\frac{u^{2}}{2}\right) L_{m_{\lambda}}\left(u^{2}\right) \int_{0}^{2 \pi} d \theta \exp \left(i s_{f}|w u| \sin \theta\right) \\
=\frac{1}{2 \pi} \int_{0}^{\infty} d|u||u| \exp \left(-\frac{u^{2}}{2}\right) L_{m_{\lambda}}\left(u^{2}\right) J_{0}(|w u|) \\
=\frac{(-1)^{m_{\lambda}}}{2 \pi} \exp \left(-\frac{w^{2}}{2}\right) L_{m_{\lambda}}\left(w^{2}\right)
\end{aligned}
$$

donde hemos usado las relaciones

$$
\int_{0}^{2 \pi} d \theta \exp ( \pm i y \sin \theta)=2 \pi J_{0}(y)
$$

y

$$
\int_{0}^{\infty} d x x^{v+1} e^{-\beta x^{2}} L_{n}^{v}\left(\alpha x^{2}\right) J_{v}(x y)=\frac{(1-\alpha / \beta)^{n}}{(2 \beta)^{v+1}} y^{v} e^{-y^{2} /(4 \beta)} L_{n}^{v}\left[\frac{\alpha y^{2}}{4 \beta(\alpha-\beta)}\right],
$$

siendo $J_{v}(x)$ las funciones de Bessel de primer tipo. Ahora, teniendo en cuenta la ecuación (F8), junto con la propiedad de ortogonalidad de los polinomios de Laguerre, encontramos

$$
\begin{aligned}
\tilde{K}_{k k^{\prime} m}^{\lambda, f(0)=} & (-1)^{k+k^{\prime}} \frac{B_{f}^{3}}{128 \pi^{4}} \int_{0}^{\infty} d|w||w| \exp \left(-w^{2}\right) L_{m_{\lambda}}\left(w^{2}\right) \int_{0}^{\infty} d|v||v| \exp \left(-v^{2}\right) \times \\
& L_{k_{\lambda}}\left(v^{2}\right) \int_{0}^{2 \pi} d \psi L_{k_{\lambda}^{\prime}}\left(v^{2}+w^{2}+2|v w| \cos \psi\right) \exp \left[-|v w| \exp \left(i s_{f} \psi\right)\right] \\
= & (-1)^{k+k^{\prime}} \frac{B_{f}^{3}}{64 \pi^{3}} \int_{0}^{\infty} d|w||w| \exp \left(-w^{2}\right) L_{m_{\lambda}}\left(w^{2}\right) L_{k_{\lambda}^{\prime}}\left(w^{2}\right) \times \\
& \int_{0}^{\infty} d|v||v| \exp \left(-v^{2}\right) L_{k_{\lambda}}\left(v^{2}\right) L_{k_{\lambda}^{\prime}}\left(v^{2}\right) \\
= & \frac{B_{f}^{3}}{256 \pi^{3}} \delta_{k k^{\prime}} \delta_{k^{\prime} m} .
\end{aligned}
$$


Para la integral $\tilde{K}_{k k^{\prime} m}^{f(1)}$ usamos el resultado en la ecuación (G6) y luego cambiamos a las nuevas variables $\bar{v}=-v$ y $\bar{w}=w+v$. Obtenemos

$$
\begin{aligned}
\tilde{K}_{k k^{\prime} m}^{f(1)}= & (-1)^{k+k^{\prime}} \frac{B_{f}^{4}}{256 \pi^{4}} \int_{0}^{\infty} d|\bar{w}| \bar{w}^{2} \exp \left(-\bar{w}^{2}\right) L_{k^{\prime}-1}^{1}\left(\bar{w}^{2}\right) \int_{0}^{\infty} d|\bar{v}| \bar{v}^{2} \exp \left(-\bar{v}^{2}\right) \times \\
& L_{k-1}^{1}\left(\bar{v}^{2}\right) \int_{0}^{2 \pi} d \psi \sin \psi L_{m_{+}}\left(\bar{v}^{2}+\bar{w}^{2}+2|\bar{v} \bar{w}| \cos \psi\right) \exp \left[-|\bar{v} \bar{w}| \exp \left(-i s_{f} \psi\right)\right] \\
= & (-1)^{k+k^{\prime}} i s_{f} \frac{B_{f}^{4}}{256 \pi^{3}}\left[\frac{1}{m_{+}+1} \int_{0}^{\infty} d|\bar{w}||\bar{w}|^{3} \exp \left(-\bar{w}^{2}\right) L_{k^{\prime}-1}^{1}\left(\bar{w}^{2}\right) L_{m_{+}}^{1}\left(\bar{w}^{2}\right) \times\right. \\
& \left.\int_{0}^{\infty} d|\bar{v}||\bar{v}|^{3} \exp \left(-\bar{v}^{2}\right) L_{k-1}^{1}\left(\bar{v}^{2}\right) L_{m_{+}}^{1}\left(\bar{v}^{2}\right)-\left(m_{+} \longleftrightarrow m_{+}-1\right)\right] \\
= & i s_{f} k \frac{B_{f}^{4}}{1024 \pi^{3}} \delta_{k k^{\prime}}\left(\delta_{m_{+} k-1}-\delta_{m_{+} k}\right),
\end{aligned}
$$

donde hemos utilizado la relación en (F10). Finalmente, notando que

$$
\sum_{m=0}^{\infty} s_{f}\left(\delta_{m_{+} k-1}-\delta_{m_{+} k}\right) g_{m, q_{\|}}^{+, f}=g_{k, q_{\|}}^{-, f}-g_{k, q_{\|}}^{+, f},
$$

a partir de las ecuaciones (G1), (G9) y (G10) podemos llegar a la expresión (5.66). 



\section{Bibliografía}

[1] F. Close, "Introduction to Quarks and Partons" (Academic Press, London, 1979); F. Halzen y A. Martin, "Quarks and leptons: An Introductory Course in Modern Particle Physics" (John Wiley \& Sons, Ney York, 1979); D. Griffiths, "Introduction to Elementary Particles", 2nd ed. (Wiley-VCH, Weinheim, Germany, 2008); W.N. Cottingham y D.A. Greenwood, "An Introduction to the Sandard Model of Particle Physics" (Cambridge University Press, Cambridge, 2007); John F. Donoghue, Eugene Golowich y Barry R. Holstein. "Dynamics of the Standard Model" (Cambridge University Press,1992).

[2] F. J. Ynduráin, "The theory of quarks and gluon interactions" (Springer-Verlag, Heilderberg, 1999); W.N. Cottingham y D.A. Greenwood.

[3] S. Weinberg, Phys. Rev. Lett. 31, 494 (1973); H. Fritzsch, M. Gell-Mann y H. Leutwyler, Phys. Lett. 47B, 365 (1973).

[4] J. C. Collins y M. J. Perry, Phys. Rev. Lett. 34, 1353 (1975).

[5] N. Cabibbo y G. Parisi, Phys. Lett. 59B, 67 (1975).

[6] J. Cleymans, R. V. Gavai y E. Suhonen, Phys. Rept. 130, 217 (1986).

[7] H. Meyer-Ortmanns, Rev. Mod. Phys. 68, 473 (1996).

[8] T. Schafer, Quark Matter, in: A.B. Santra et al. (Eds.), "Quarks and Mesons", Proc. of the BARC workshop on Quarks and Mesons, Bhabba Atomic Research Center, Mumbai, India (2003) (Narosa Publishing House, New Delhi, 2004).

[9] S. Aoki et al. [JLQCD Collaboration], Phys. Rev. D 57, 3910 (1998).

[10] Y. Aoki, Z. Fodor, S. D. Katz y K. K. Szabo, Phys. Lett. B 643, 46 (2006); Y. Aoki, S. Borsanyi, S. Durr, Z. Fodor, S. D. Katz, S. Krieg y K. K. Szabo, JHEP 0906, 088 (2009).

[11] N. K. Glendenning, “Compact Stars: Nuclear Physics, Particle Physics and General Relativity", 2nd Edition, (Springer Verlag, NY, 2000); S. L. Shapiro y S. A. Teukolsky, 
"Black Holes, White Dwarfs and Neutron Stars: The Physics of Compact Objects", (Wiley-Interscience, NY, 1983).

[12] D. Blaschke, N. K. Glendenning y A. Sedrakian, Lect. Notes Phys. 578, 1 (2001).

[13] Ver por ejemplo: L. Ruan, Front. Phys. China 5, 205 (2010) [arXiv:1007.2882 [nucl-ex]]. Para más detalles ver el sitio http://www.bnl.gov/RHIC/.

[14] R. Preghenella [ALICE Collaboration], EPJ Web Conf. 49, 02003 (2013). Para más detalles ver el sitio http:/ /aliceinfo.cern.ch/.

[15] G. Rosner, Nucl. Phys. Proc. Suppl. 167, 29 (2007). Para más detalles ver el sitio http://www.fair-center.eu/.

[16] G. Trubnikov, A. Kovalenko, V. Kekelidze, I. Meshkov, R. Lednicky, A. Sissakian y A. Sorin, PoS ICHEP 2010, 523 (2010). Para más detalles ver el sitio http://nucloweb.jinr.ru/nica/index1.htm.

[17] R. Nouicer, Eur. Phys. J. Plus 131, no. 3, 70 (2016).

[18] I. Arsene et al. [BRAHMS Collaboration], Nucl. Phys. A 757, 1 (2005).

[19] B. B. Back et al., Nucl. Phys. A 757, 28 (2005).

[20] F. Karsch y E. Laermann, Phys. Rev. D 50, 6954 (1994).

[21] F. Karsch, Lect. Notes Phys. 583, 209 (2002).

[22] W. Greiner, S. Schramm y E. Stein, "Quantum Chromodynamics", (Springer, Second Edition, 2002).

[23] C. Itzykson y J.B. Zuber "Quantum Field Theory" (McGraw-Hill Interna- tional Book Company, 1980).

[24] Y. Nambu y G. Jona-Lasinio, Phys. Rev. 122, 345 (1961).

[25] Y. Nambu y G. Jona-Lasinio, Phys. Rev. 124, 246 (1961).

[26] U. Vogl y W. Weise, Prog. Part. Nucl. Phys. 27, 195 (1991).

[27] T. Hatsuda y T. Kunihiro, Phys. Rept. 247, 221 (1994).

[28] S. P. Klevansky, Rev. Mod. Phys. 64, 649 (1992).

[29] K. Kashiwa, Phys. Rev. D 83117901 (2011).

[30] M. Buballa, Phys. Rept. 407, 205 (2005). 
[31] A. M. Polyakov, Phys. Lett. 72B, 477 (1978).

[32] G. 't Hooft, Nucl. Phys. B 138, 1 (1978); G. 't Hooft, Nucl. Phys. B 153, 141 (1979).

[33] P. N. Meisinger y M. C. Ogilvie, Phys. Lett. B 379, 163 (1996); K. Fukushima, Phys. Lett. B 591, 277 (2004); E. Megias, E. Ruiz Arriola y L. L. Salcedo, Phys. Rev. D 74, 065005 (2006).

[34] C. Ratti, M. A. Thaler y W. Weise, Phys. Rev. D 73, 014019 (2006).

[35] R. D. Pisarski, Phys. Rev. D 62, 111501 (2000).

[36] T. Schäfer y E. V. Shuryak, Rev. Mod. Phys. 70, 323 (1998).

[37] C. D. Roberts y A. G. Williams, Prog. Part. Nucl. Phys. 33, 477 (1994); C. D. Roberts y S. M. Schmidt, Prog. Part. Nucl. Phys. 45, S1 (2000).

[38] S. M. Schmidt, D. Blaschke y Y. L. Kalinovsky, Phys. Rev. C 50, 435 (1994).

[39] E. Ruiz Arriola y L. L. Salcedo, Phys. Lett. B 450, 225 (1999).

[40] G. Ripka, Nucl. Phys. A 683, 463 (2001); R. S. Plant y M. C. Birse, Nucl. Phys. A 703, 717 (2002).

[41] R. D. Bowler y M. C. Birse, Nucl. Phys. A 582, 655 (1995).

[42] D. Kharzeev, K. Landsteiner, A. Schmitt y H. U. Yee, Lect. Notes Phys. 871, 1 (2013).

[43] D. E. Kharzeev, L. D. McLerran y H. J. Warringa, Nucl. Phys. A 803, 227 (2008); V. Skokov, A. Y. Illarionov y V. Toneev, Int. J. Mod. Phys. A 24, 5925 (2009); V. Voronyuk, V. D. Toneev, W. Cassing, E. L. Bratkovskaya, V. P. Konchakovski y S. A. Voloshin, Phys. Rev. C 83, 054911 (2011).

[44] R. C. Duncan y C. Thompson, Astrophys. J. 392, L9 (1992); C. Kouveliotou et al., Nature 393, 235 (1998).

[45] D. Lai y S. L. Shapiro, Astrophys. J. 383, 745 (1991).

[46] D. Bandyopadhyay, S. Chakrabarty y S. Pal, Phys. Rev. Lett. 79, 2176 (1997).

[47] E. J. Ferrer, V. de la Incera, J. P. Keith, I. Portillo y P. L. Springsteen, Phys. Rev. C 82, 065802 (2010).

[48] V. P. Gusynin, V. A. Miransky y I. A. Shovkovy, Phys. Rev. Lett. 73, 3499 (1994). Erratum: [Phys. Rev. Lett. 76, 1005 (1996)]. 
[49] F. Preis, A. Rebhan y A. Schmitt, JHEP 1103, 033 (2011).

[50] D. Ebert, K. G. Klimenko, M. A. Vdovichenko y A. S. Vshivtsev, Phys. Rev. D 61, 025005 (2000).

[51] D. Ebert y K. G. Klimenko, Nucl. Phys. A 728, 203 (2003).

[52] J. Goldstone, Nuovo Cim. 19, 154 (1961).

[53] J. Goldstone, A. Salam y S. Weinberg, Phys. Rev. 127, 965 (1962).

[54] Steven Weinberg. "The Quantum Theory of Fields, Volume 2" (Cambridge University Press, 1995).

[55] J. Gasser and H. Leutwyler, Annals Phys. 158, 142 (1984).

[56] D. J. Gross y F. Wilczek, Phys. Rev. D 8, 3633 (1973).

[57] H. D. Politzer, Phys. Rept. 14, 129 (1974).

[58] G. Ripka, "Quarks bound by chiral fields" (Oxford University Press, Oxford, 1997).

[59] M. B. Parappilly, P. O. Bowman, U. M. Heller, D. B. Leinweber, A. G. Williams y J. B. Zhang, Phys. Rev. D 73, 054504 (2006).

[60] D. Blaschke, Y. L. Kalinovsky, G. Roepke, S. M. Schmidt y M. K. Volkov, Phys. Rev. C 53, 2394 (1996).

[61] D. Diakonov y V. Y. Petrov, Sov. Phys. JETP 62, 204 (1985); Sov. Phys. JETP 62, 431 (1985); Nucl. Phys. B 245, 259 (1984); Nucl. Phys. B 272, 457 (1986); D. Diakonov, V. Y. Petrov y P. V. Pobylitsa, Nucl. Phys. B 306, 809 (1988).

[62] S. Furui y H. Nakajima, Phys. Rev. D 73, 074503 (2006).

[63] S. Noguera y N. N. Scoccola, Phys. Rev. D 78, 114002 (2008).

[64] R. S. Plant y M. C. Birse, Nucl. Phys. A 628, 607 (1998).

[65] S. Noguera, Int. J. Mod. Phys. E 16, 97 (2007).

[66] T. Hell, S. Rossner, M. Cristoforetti y W. Weise, Phys. Rev. D 79, 014022 (2009); Phys. Rev. D 81, 074034 (2010).

[67] A. E. Radzhabov, D. Blaschke, M. Buballa y M. K. Volkov, Phys. Rev. D 83, 116004 (2011).

[68] D. Gomez Dumm, A. G. Grunfeld y N. N. Scoccola, Phys. Rev. D 74, 054026 (2006). 
[69] D. Gomez Dumm, S. Noguera y N. N. Scoccola, Phys. Lett. B 698, 236 (2011).

[70] M.F. Izzo Villafañe, D. Gómez Dumm y N. N. Scoccola, Phys. Rev. D 94, 054003 (2016).

[71] M.F. Izzo Villafañe y D. Gómez Dumm, J. Phys. Conf. Ser. 706, 042013 (2016).

[72] D. Ebert y H. Reinhardt, Nucl. Phys. B 271, 188 (1986).

[73] V. Bernard, U. G. Meissner y A. A. Osipov, Phys. Lett. B 324, 201 (1994); V. Bernard, A. H. Blin, B. Hiller, Y. P. Ivanov, A. A. Osipov y U. G. Meissner, Ann. Phys. 249, 499 (1996).

[74] A. H. Blin, B. Hiller y M. Schaden, Z. Phys. A 331, 75 (1988).

[75] K.A. Olive et al. (Particle Data Group), Chin. Phys. C 38, 090001 (2014).

[76] P. O. Bowman, U. M. Heller y A. G. Williams, Phys. Rev. D 66, 014505 (2002); P. O. Bowman, U. M. Heller, D. B. Leinweber y A. G. Williams, Nucl. Phys. B (Proc. Suppl.) 119, 323 (2003).

[77] Y. B. He, J. Hufner, S. P. Klevansky y P. Rehberg, Nucl. Phys. A 630, 719 (1998).

[78] P. Zhuang, J. Hufner y S. P. Klevansky, Nucl. Phys. A 576, 525 (1994).

[79] H. Hansen, W. M. Alberico, A. Beraudo, A. Molinari, M. Nardi y C. Ratti, Phys. Rev. D 75, 065004 (2007).

[80] Ver por ejemplo C. McNeile, Phys. Lett. B 619, 124 (2005), y las referencias ahí presentes.

[81] J. P. Carlomagno, D. Gomez Dumm y N. N. Scoccola, Phys. Rev. D 88, 074034 (2013).

[82] T. Hell, K. Kashiwa y W. Weise, Phys. Rev. D 83, 114008 (2011).

[83] G. S. Bali, F. Bruckmann, G. Endrodi, Z. Fodor, S. D. Katz, S. Krieg, A. Schafer y K. K. Szabo, JHEP 1202, 044 (2012).

[84] G. S. Bali, F. Bruckmann, G. Endrodi, Z. Fodor, S. D. Katz y A. Schafer, Phys. Rev. D 86, 071502 (2012).

[85] J. O. Andersen, W. R. Naylor y A. Tranberg, Rev. Mod. Phys. 88, 025001 (2016).

[86] D. E. Kharzeev, K. Landsteiner, A. Schmitt y H. U. Yee, Lect. Notes Phys. 871, 1 (2013).

[87] V. A. Miransky y I. A. Shovkovy, Phys. Rept. 576, 1 (2015).

[88] A. Ayala, M. Loewe, A. J. Mizher y R. Zamora, Phys. Rev. D 90, 036001 (2014). 
[89] R. L. S. Farias, K. P. Gomes, G. I. Krein y M. B. Pinto, Phys. Rev. C 90, 025203 (2014).

[90] V.P. Pagura, D. Gomez Dumm, S. Noguera y N.N. Scoccola, Phys. Rev. D 95, 034013 (2017).

[91] D. Gomez Dumm, M. F. Izzo Villafañe, S. Noguera, V. P. Pagura y N. N. Scoccola, arXiv:1709.04742 [hep-ph].

[92] B. B. Brandt, G. Bali, G. Endrödi y B. Glässle, PoS LATTICE 2015, 265 (2016).

[93] G. S. Bali, B. B. Brandt, G. Endrodi y B. Glaessle, arXiv:1707.05600 [hep-lat].

[94] S. Fayazbakhsh y N. Sadooghi, Phys. Rev. D 88, 065030 (2013).

[95] S. Fayazbakhsh, S. Sadeghian y N. Sadooghi, Phys. Rev. D 86, 085042 (2012).

[96] S. S. Avancini, W. R. Tavares y M. B. Pinto, Phys. Rev. D 93, 014010 (2016).

[97] S. S. Avancini, R. L. S. Farias, M. Benghi Pinto, W. R. Tavares y V. S. Timóteo, Phys. Lett. B 767, 247 (2017).

[98] J. O. Andersen, JHEP 1210, 005 (2012).

[99] N. O. Agasian y I. A. Shushpanov, JHEP 0110, 006 (2001).

[100] V. D. Orlovsky y Y. A. Simonov, JHEP 1309, 136 (2013).

[101] M. A. Andreichikov, B. O. Kerbikov, E. V. Luschevskaya, Y. A. Simonov y O. E. Solovjeva, JHEP 1705, 007 (2017).

[102] V. I. Ritus, Sov. Phys. JETP 48, 788 (1978).

[103] P. Watson y H. Reinhardt, Phys. Rev. D 89, 045008 (2014).

[104] D. P. Menezes, M. Benghi Pinto, S. S. Avancini, A. Perez Martinez y C. Providencia, Phys. Rev. C 79, 035807 (2009).

[105] D. Gomez Dumm, M.F. Izzo Villafañe y N.N. Scoccola, arXiv:1710.08950 [hep-ph].

[106] G. 't Hooft, Phys. Rev. Lett. 37, 8 (1976); G. 't Hooft, Phys. Rev. D 14, 3432 (1976). Erratum: [Phys. Rev. D 18, 2199 (1978)].

[107] D. Blaschke, M. Buballa, A. E. Radzhabov y M. K. Volkov, Yad. Fiz. 71, 2012 (2008) [Phys. Atom. Nucl. 71, 1981 (2008)].

[108] G. A. Contrera, D. Gomez Dumm y N. N. Scoccola, Phys. Lett. B 661, 113 (2008). 
[109] G. A. Contrera, D. Gomez Dumm y N. N. Scoccola, Phys. Rev. D 81, 054005 (2010).

[110] T. Hell, S. Rossner, M. Cristoforetti y W. Weise, Phys. Rev. D 81, 074034 (2010).

[111] M.F. Izzo Villafañe, J.P. Carlomagno, D.G. Dumm y N.N. Scoccola, Int. J. Mod. Phys. Conf. Ser. 45, 1760056 (2017).

[112] S. Roessner, C. Ratti y W. Weise, Phys. Rev. D 75, 034007 (2007).

[113] O. Scavenius, A. Dumitru y J. T. Lenaghan, Phys. Rev. C 66, 034903 (2002).

[114] B.-J. Schaefer, J. M. Pawlowski y J. Wambach, Phys. Rev. D 76 , 074023 (2007); B.-J. Schaefer, M. Wagner y J. Wambach, Phys. Rev. D 81 , 074013 (2010).

[115] A. Dumitru, R. D. Pisarski y D. Zschiesche, Phys. Rev. D 72, 065008 (2005).

[116] D. Gomez Dumm y N. N. Scoccola, Phys. Rev. C 72, 014909 (2005).

[117] M. Ferreira, P. Costa, O. Lourenço, T. Frederico y C. Providência, Phys. Rev. D 89, 116011 (2014).

[118] T. Hell, K. Kashiwa y W. Weise, J. Mod. Phys. 4, 644 (2013).

[119] D. B. Blaschke, D. Gomez Dumm, A. G. Grunfeld, T. Klahn y N. N. Scoccola, Phys. Rev. C 75, 065804 (2007); M. Orsaria, H. Rodrigues, F. Weber y G. A. Contrera, Phys. Rev. D 87, 023001 (2013).

[120] I. S. Gradshteyn y I. M. Ryzhik, Table of Integrals, Series, and Products, (Academic Press, London, 1996). 